\title{
An investigation of intrinsic brain properties through multimodal imaging in humans
}

by

\author{
Niall Duncan
}

A thesis submitted to the Faculty of Graduate and Postdoctoral Affairs in partial fulfillment of the requirements for the degree of Doctor of

Philosophy in Biology with a specialisation in Neuroscience

Carleton University

Ottawa, Ontario

(C)2013

Niall Duncan 
Library and Archives

Canada

Published Heritage

Branch

395 Wellington Street

Ottawa ON K1A ON4

Canada
Bibliothèque et

Archives Canada

Direction du

Patrimoine de l'édition

395 , rue Wellington

Ottawa ON K1A ON4

Canada
Your file Votre référence

ISBN: 978-0-494-94213-0

Our file Notre référence

ISBN: $978-0-494-94213-0$
NOTICE:

The author has granted a nonexclusive license allowing Library and Archives Canada to reproduce, publish, archive, preserve, conserve, communicate to the public by telecommunication or on the Internet, loan, distrbute and sell theses worldwide, for commercial or noncommercial purposes, in microform, paper, electronic and/or any other formats.

The author retains copyright ownership and moral rights in this thesis. Neither the thesis nor substantial extracts from it may be printed or otherwise reproduced without the author's permission.
AVIS:

L'auteur a accordé une licence non exclusive permettant à la Bibliothèque et Archives Canada de reproduire, publier, archiver, sauvegarder, conserver, transmettre au public par télécommunication ou par l'Internet, prêter, distribuer et vendre des thèses partout dans le monde, à des fins commerciales ou autres, sur support microforme, papier, électronique et/ou autres formats.

L'auteur conserve la propriété du droit d'auteur et des droits moraux qui protege cette thèse. $\mathrm{Ni}$ la thèse ni des extraits substantiels de celle-ci ne doivent être imprimés ou autrement reproduits sans son autorisation.
In compliance with the Canadian Privacy Act some supporting forms may have been removed from this thesis.

While these forms may be included in the document page count, their removal does not represent any loss of content from the thesis.
Conformément à la loi canadienne sur la protection de la vie privée, quelques formulaires secondaires ont été enlevés de cette thèse.

Bien que ces formulaires aient inclus dans la pagination, il n'y aura aucun contenu manquant. 


\section{Abstract}

For much of the past 150 years the main paradigm in brain research has been one of localisation - the mapping of processes to particular regions. More recently, a shift towards a more network-based approach has taken place. One of the factors supporting this shift has been an appreciation of the importance of ongoing, stimulus-independent, activity to mental processes. Such ongoing activity has been productively studied at the functional and anatomical network level, as well as within isolated regions. However, to date, less has been established regarding the biochemical underpinnings of such activity and the links between the different levels of investigation (structural, functional, process). In order to investigate these points, multiple magnetic resonance imaging (MRI) techniques were combined with $\mathrm{GABA}_{\mathrm{A}}$ receptor positron emission tomography and electroencephalography in a set of studies in healthy humans. Using magnetic resonance spectroscopy, diffusion tensor imaging, and functional MRI, it was shown firstly that medial prefrontal cortex (mPFC) glutamate levels correlate with structural and functional connectivity to multiple cortical and subcortical regions during both rest and task. It was further shown that glutamate in this region is correlated with the signal dynamics of the ongoing activity. Secondly, it was shown that cortical $\mathrm{GABA}_{\mathrm{A}}$ receptor distribution follows similar topologies to structural and functional connectivity, fitting the description of "small-worldness". It was further shown that the distribution can be decomposed into distinct networks that correspond to known functional and structural ones. Thirdly, using fMRI and DTI, it was shown that the structural network profile of the insula predicts its activity during a specific task and that the different structural networks identified corresponded well with previously established ones. Finally, it was shown that ongoing activity dynamics in adulthood are

related to early-life stress, and that the dynamical differences seen across individuals were related to mPFC glutamate levels. It was further shown that these early-life stress related changes are correlated with altered neural responses to aversive stimuli. Taken together, the results presented provide initial biochemical links between structural and functional networks and that these can be related to individual differences in psychological processes. 


\section{Acknowledgements}

My thanks, firstly, to Dr. Georg Northoff and my colleagues at the Mind, Brain Imaging and Neuroethics group for their input and support. I am grateful also to Dr. James Cheetham and my thesis committee for their constructive input.

Thanks are due also to the technical staff at the various scanning centres where this work was carried out for their contribution, along with the people who volunteered as participants.

Finally, many thanks to my family and friends, whose presence from afar was invaluable. 


\section{Contributions}

A number of people have contributed to the research described in this work. Their exact contributions to each paper are outlined here.

Imaging data was acquired at three sites: the Universitätsklinik für Neurologie, Otto-vonGuericke University, Magdeburg, Germany; the McConnell Brain Imaging Centre, Montreal Neurological Institute, McGill University Montreal, Canada; and the Unite de Neuroimagerie Fonctionnelle, Université de Montréal, Montréal, Canada. Data analysis was done at the Universitätsklinik für Neurologie, Otto-von-Guericke University, Magdeburg, Germany, and at the Mind, Brain Imaging and Neuroethics Unit, Institute of Mental Health Research, University of Ottawa, Ottawa, Canada.

\section{Glutamate in the mPFC predicts resting-state cortical-subcortical functional connectivity}

Niall W. Duncan - Designed study; acquired data; conceived of and carried out analysis; wrote manuscript.

Christine Wiebking ${ }^{1,2}$ - Designed study; acquired data.

Brice Tiret $^{3}$ - MRS processing.

Dr. Dave J. Hayes ${ }^{1}$ - Designed study; acquired data.

Dr. Oliver Lyttleton ${ }^{1}$ - Provided technical input.

Dr. Malgorzata Marjańska ${ }^{4}$ - Provided technical input.

Dr. Julien Doyon ${ }^{3}$ - Provided equipment. 
Dr. Georg Northoff ${ }^{1}$ - Designed study; provided conceptual input.

Involvement of glutamate in rest-stimulus interaction between perigenual and supragenual anterior cingulate cortex: A combined FMRI-MRS study

Human Brain Mapping, 2011, 32(12):2172-82

Niall W. Duncan - Conceived of and carried out analysis; wrote manuscript.

Dr. Björn Enzi ${ }^{5}$ - Designed study; acquired data.

Christine Wiebking ${ }^{1,2}$ - Designed study; acquired data.

Dr. Georg Northoff ${ }^{1}$ - Designed study; provided conceptual input.

Glutamate related entropy in the mPFC alters between eyes-open and eyes-closed rest

Niall W. Duncan - Designed study; acquired MRI data; conceived of and carried out analysis; wrote manuscript.

Dr. Takashi Nakao ${ }^{6}$ - Acquired and pre-processed EEG data.

Christine Wiebking ${ }^{1,2}$ - Acquired MRI data.

Brice Tiret $^{3}$ - MRS processing.

Dr. Malgorzata Marjańska ${ }^{4}$ - Provided technical input.

Dr. Georg Northoff ${ }^{1}$ - Designed study; provided conceptual input.

Grey matter density and $\mathrm{GABA}_{A}$ binding potential show a positive linear relationship across cortical regions

Neuroscience, in press 
Niall W. Duncan - Designed study; acquired data; conceived of and carried out analysis; wrote manuscript.

Paul Gravel ${ }^{7}$ - PET reconstruction; provided technical input.

Christine Wiebking ${ }^{1,2}$ - Designed study; acquired data.

Dr. Oliver Lyttleton ${ }^{1}$ - Provided technical input.

Dr. Andrew J. Reader ${ }^{7}$ - PET reconstruction; provided technical input.

Dr. Georg Northoff ${ }^{1}$ - Designed study.

\section{Cortical $\mathrm{GABA}_{\mathrm{A}}$ receptor binding potential patterns display small-world characteristics}

Niall W. Duncan - Designed study; acquired data; conceived of and carried out analysis; wrote manuscript.

Christine Wiebking ${ }^{1,2}$ - Designed study; acquired data.

Paul Gravel ${ }^{7}$ - PET reconstruction; provided technical input.

Dr. Andrew J. Reader ${ }^{7}$ - PET reconstruction.

Dr. Georg Northoff ${ }^{1}$ - Designed study.

\section{Differential insula structural connectivity patterns predict BOLD responses to intero- and exteroception}

Niall W. Duncan - Designed study; acquired data; conceived of and carried out analysis; wrote manuscript.

Christine Wiebking ${ }^{1,2}$ - Designed study; acquired data.

Dr. Georg Northoff ${ }^{1}$ - Designed study. 


\section{Reported early-life stress modulates resting-state glutamate and entropy with concomitant effects on aversion-specific brain responses}

Niall W. Duncan - Designed study; acquired data; conceived of and carried out analysis; wrote manuscript.

Dr. Dave J. Hayes ${ }^{1}$ - Designed study; acquired data.

Christine Wiebking ${ }^{1,2}$ - Designed study; acquired data.

Brice Tiret $^{3}$ - MRS processing.

Karin Pietruska ${ }^{8}$ - Acquired data.

Dr. Pierre Rainville ${ }^{8}$ - Provided expertise on aversive electrical stimulation during fMRI and on the related experimental design, and revised the manuscript.

Dr. Malgorzata Marjańska ${ }^{4}$ - Provided technical input.

Dr. Georg Northoff ${ }^{1}$ - Designed study; provided conceptual input.

\section{Affiliations}

${ }^{1}$ Mind, Brain Imaging and Neuroethics Research Unit, Institute of Mental Health Research, University of Ottawa, Ottawa, Canada

${ }^{2}$ Department of Biology, Freie Universität Berlin, Berlin, Germany

${ }^{3}$ Functional Neuroimaging Unit and Department of Psychology, Lniversité de Montréal, Montréal, Canada

${ }^{4}$ Center for Magnetic Resonance Research and Department of Radiology, University of Minnesota, Minneapolis, USA

${ }^{5}$ Department of Psychiatry, Psychotherapy and Preventive Medicine, LWL University Hospital Bochum, Ruhr-University Bochum, Germany 
${ }^{6}$ Institute of Biomedical \& Health Sciences, Hiroshima University, Hiroshima, Japan

7 McConnell Brain Imaging Centre, Montréal Neurological Institute, McGill University Montréal, Canada

${ }^{8}$ Faculté de médecine dentaire, Université de Montréal, Montréal, Canada

\section{Contribution statement}

I can confirm that Niall Duncan was responsible for all of the work described in this thesis, as laid out in the preceding contribution descriptions. He planned and conducted all research and analysis. All material included here was written by him.

Dr. Georg Northoff, M.D., Ph.D.

Research Director, Mind Brain Imaging and Neuroethics Unit

Institute of Mental Health Research, University of Ottawa

Adjunct Research Professor, Department of Neuroscience

Carleton University 


\section{Contents}

1 General introduction 1

1.1 The brain as a dynamic network . . . . . . . . . . . . . . 1

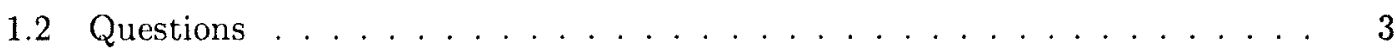

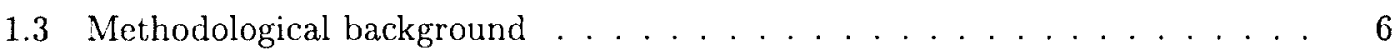

2 Glutamate in the mPFC predicts resting-state cortical-subcortical functional connectivity 11

2.1 Abstract . . . . . . . . . . . . . . . . . . . . 11

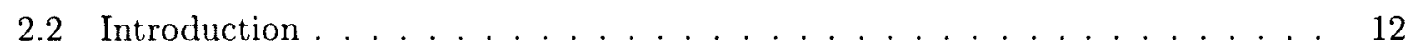

2.3 Materials and methods . . . . . . . . . . . . . . . . . . . 13

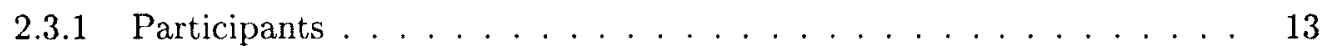

$2.3 .2 \quad$ MRS . . . . . . . . . . . . . . . . . . 14

2.3 .3 ROIs . . . . . . . . . . . . . . . . . . . . . 17

2.3 .4 fMRI . . . . . . . . . . . . . . . . . . 17

2.3 .5 DTI . . . . . . . . . . . . . . . . . . . 21

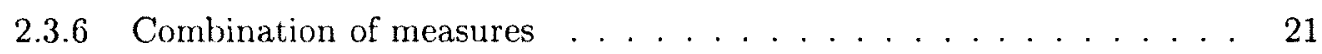

2.4 Results. . . . . . . . . . . . . . . . . . . . 22

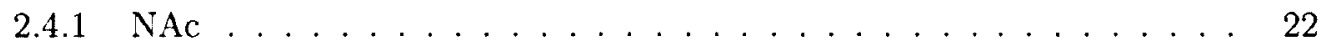

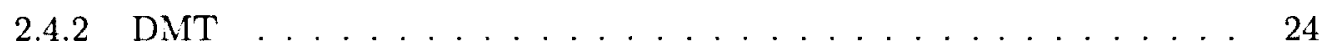

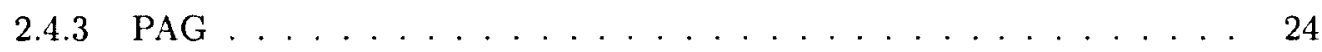

2.5 Discussion . . . . . . . . . . . . . . . . . . 26

2.5.1 mPFC glutamate and subcortical functional connectivity . . . . 26

2.5.2 Implications for MDD and schizophrenia . . . . . . . . . . 30

2.5 .3 Limitations \& conclusion . . . . . . . . . . . . . . . 31

2.6 Acknowledgements . . . . . . . . . . . . . . . . . . . . . 32

3 Involvement of glutamate in rest-stimulus interaction between perigenual and supragenual anterior cingulate cortex 
3.1 Abstract . . . . . . . . . . . . . . . . . . . . . 33

3.2 Introduction . . . . . . . . . . . . . . . . . 33

3.3 Methods . . . . . . . . . . . . . . . . . . 35

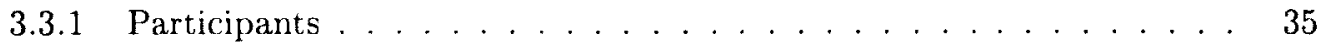

3.3 .2 Experimental paradigm . . . . . . . . . . . . 36

3.3 .3 fMRI data acquisition and analysis . . . . . . . . . . 38

3.3.4 MRS data acquisition and analysis . . . . . . . . . . . 40

3.4 Results . . . . . . . . . . . . . . . . . . . . . . . 42

3.4 .1 Positive signal changes . . . . . . . . . . . . . . 42

3.4 .2 Negative signal changes . . . . . . . . . . . . . . 43

3.4 .3 Inter-regional connectivity . . . . . . . . . . . . . 43

3.4.4 Resting state Glx and regional signal changes . . . . . . . . . 45

3.5 Discussion . . . . . . . . . . . . . . . . . . . . 48

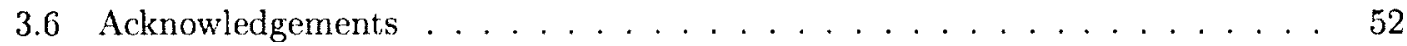

4 Glutamate and intra-regional activity 53

5 Glutamate related entropy in the MPFC alters between eyes-open and eyesclosed rest $\quad 59$

5.1 Abstract . . . . . . . . . . . . . . . . . . . . 59

5.2 Introduction . . . . . . . . . . . . . . . . . . 59

5.3 Methods . . . . . . . . . . . . . . . . . . . 61

5.3 .1 Dynamic measures . . . . . . . . . . . . . . . 61

5.3 .2 Participants . . . . . . . . . . . . . . . . 62

5.3 .3 EEG acquisition and analysis ................ 63

5.3 .4 MRI . . . . . . . . . . . . . . . . . . . . 64

5.4 Results . . . . . . . . . . . . . . . . . . . . 67

5.4 .1 EEG - entropy EO \& EC . . . . . . . . . . . . . 67

5.4 .2 fMRI - entropy EO \& EC . . . . . . . . . . . . . 68

5.4 .3 MRI - entropy and biochemistry . . . . . . . . . . . 72

5.5 Discussion . . . . . . . . . . . . . . . . . . . . . . . 72

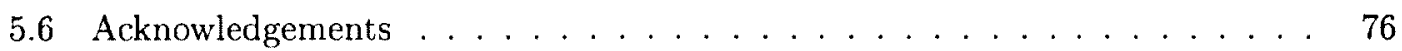

6 Considerations for ${ }^{18} \mathrm{~F}$-flumazenil PET imaging of GABAA receptors 77

6.1 PET imaging . . . . . . . . . . . . . . . 77 
6.2 The $\mathrm{GABA}_{\mathrm{A}}$ receptor and flumazenil $\ldots \ldots \ldots \ldots \ldots$

6.3 Comparison of reference region location and volumes $\ldots \ldots \ldots \ldots$

6.4 A marker of receptors or of neurons? . . . . . . . . . . . . . . 83

7 Grey matter density and $\mathrm{GABA}_{A}$ binding potential show a positive linear rela$\begin{array}{ll}\text { tionship across cortical regions } & \mathbf{8 4}\end{array}$

7.1 Abstract . . . . . . . . . . . . . . . . . . . . 84

7.2 Introduction . . . . . . . . . . . . . . . . 84

7.3 Methods . . . . . . . . . . . . . . . . . . 86

7.3 .1 Participants . . . . . . . . . . . . . . 86

$7.3 .2 \quad$ MRI . . . . . . . . . . . . . . . . . . . 86

$7.3 .3 \quad$ PET . . . . . . . . . . . . . . . 86

7.3 .4 Regions of interest . . . . . . . . . . . . . . 88

7.3.5 Comparison of $\mathrm{GM}$ and $\mathrm{BP}_{\mathrm{ND}} \ldots \ldots \ldots . \ldots \ldots$

7.4 Results . . . . . . . . . . . . . . . . . . . . . . 89

$7.4 .1 \quad \mathrm{GM}$ and $\mathrm{BP}_{\mathrm{ND}}$ values $\ldots \ldots \ldots \ldots \ldots . \ldots \ldots$

7.4 .2 Global BP $\mathrm{ND}-\mathrm{GM}$ correlation . . . . . . . . . . . 89

7.4 .3 Regional $\mathrm{BP}_{\mathrm{ND}}-\mathrm{GM}$ correlation . . . . . . . . . . . 90

7.5 Discussion . . . . . . . . . . . . . . . . . . . . . . . . . 92

7.6 Acknowledgements $\ldots \ldots \ldots \ldots \ldots$

8 Cortical $\mathrm{GABA}_{\mathrm{A}}$ receptor binding potential patterns display small-world characteristics $\quad 95$

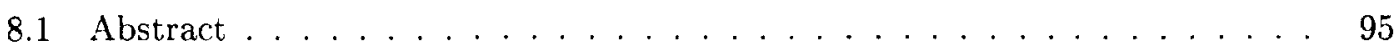

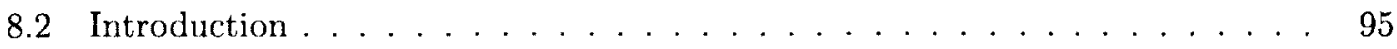

8.3 Methods . . . . . . . . . . . . . . . . . . . 97

8.3 .1 Participants . . . . . . . . . . . . . . . . 97

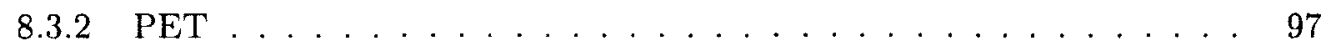

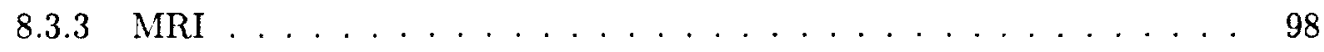

8.3 .4 Network creation . . . . . . . . . . . . . . . . 99

8.3 .5 Network metrics . . . . . . . . . . . . . . . . . . 100

8.4 Results . . . . . . . . . . . . . . . . . . . . 101

8.5 Discussion . . . . . . . . . . . . . . . . . . . 104

8.6 Acknowledgements . . . . . . . . . . . . . . . . . 108 
9 Differential insula structural connectivity patterns predict BOLD responses to $\begin{array}{lr}\text { intero- and exteroception } & 109\end{array}$

9.1 Abstract . . . . . . . . . . . . . . . . . . . . . 109

9.2 Introduction $\ldots \ldots \ldots \ldots \ldots \ldots$

9.3 Methods . . . . . . . . . . . . . . . . . . . . . . . . . . 111

9.3 .1 Participants . . . . . . . . . . . . . . . 111

9.3 .2 fMRL . . . . . . . . . . . . . . . . . . 111

9.3 .3 Regions of interest . . . . . . . . . . . . . . . . . . . . . . . . . . . . . . . . . 112

9.3 .4 DTI . . . . . . . . . . . . . . . . . . 113

9.3.5 Model construction . . . . . . . . . . . . . . 113

9.4 Results . . . . . . . . . . . . . . . . . . . . . 117

9.5 Discussion . . . . . . . . . . . . . . . . . . . . . . . 121

9.6 Acknowledgements . . . . . . . . . . . . . . . . . . . . . . . 124

10 Reported early-life stress predicts mPFC resting-state glutamate and entropy with concomitant effects on aversion-related brain responses in healthy adults $\mathbf{1 2 5}$

10.1 Abstract . . . . . . . . . . . . . . . . . . . . 125

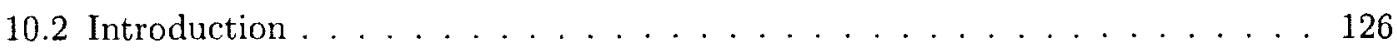

10.3 Methods . . . . . . . . . . . . . . . . . . . . 127

10.3.1 Participants . . . . . . . . . . . . . . . . 127

10.3.2 Psychological scales . . . . . . . . . . . . . . . 128

10.3 .3 MRS . . . . . . . . . . . . . . . . . . . . . . . . 129

10.3.4 fMRI parameters and pre-processing . . . . . . . . . . . . 129

10.3.5 Resting-state fMRI . . . . . . . . . . . . . . . 130

10.3 .6 Aversion task . . . . . . . . . . . . . . . . 132

10.3.7 Combination of measures . . . . . . . . . . . . . 133

10.4 Results . . . . . . . . . . . . . . . . . . . . . . . 134

10.4.1 CTQ \& biochemistry . . . . . . . . . . . . . . . 134

10.4.2 CTQ \& resting-state MRI . . . . . . . . . . . . . . . 134

10.4.3 Aversion response \& CTQ, biochemistry and resting-state fMRI . . . 135

10.5 Discussion . . . . . . . . . . . . . . . . . . . . 137

10.6 Acknowledgements . . . . . . . . . . . . . . . 145

11 Discussion $\quad 146$ 
$\begin{array}{ll}\text { A Supplementary material for Chapter } 2 & 215\end{array}$

B Supplementary material for Chapter $3 \quad 221$

$\begin{array}{ll}\text { C Supplementary material for Chapter } 7 & 226\end{array}$

$\begin{array}{ll}\text { D Supplementary material for Chapter } 9 & 229\end{array}$

E Supplementary material for Chapter $10 \quad 237$ 


\section{List of Figures}

1.1 Example timecourses and the correlational functional connectivity between them. . . . . . . . . . . . . . . . . . . 2

2.1 mPFC and left insula MRS voxels. . . . . . . . . . . . . . . 15

2.2 Sample LCModel quantification of MRS spectra. . . . . . . . . . . . 16

2.3 Target ROI locations. . . . . . . . . . . . . . . . . . . . 18

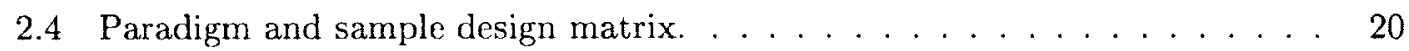

2.5 Results of FC vs glutamate and FC vs DTI analyses. . . . . . . . . 23

2.6 Overview of results. . . . . . . . . . . . . . . 27

3.1 Empathy meta-analysis overlap. . . . . . . . . . . . . . . 38

3.2 MRS voxel locations. . . . . . . . . . . . . . . . . 41

3.3 Empathy task activations and deactivations. . . . . . . . . . . . . . 44

3.4 pgACC to sgACC correlation and connectivity results. . . . . . . . 46

4.1 Correlation between fixation signals and $\mathrm{pgACC} \mathrm{Glx} / \mathrm{Cr} \ldots \ldots \ldots . \ldots 55$

4.2 SD and entropy. . . . . . . . . . . . . . . . . . . 57

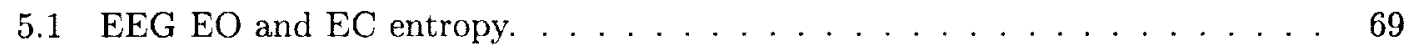

5.2 fMRI EO and EC entropy and SD . . . . . . . . . . . . 70

5.3 mPFC entropy and glutamate. . . . . . . . . . . . 73

6.1 Reference region location and sizes. . . . . . . . . . . . 81

6.2 Comparison of PVC-BP $\mathrm{ND}_{\text {and }} \mathrm{SNR}$ values. . . . . . . . . 82

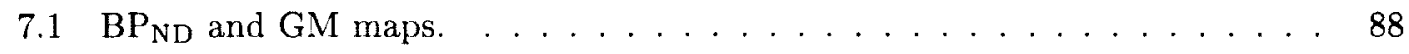

7.2 Correlation between regional $\mathrm{BP}_{\mathrm{ND}}$ and $\mathrm{GM} . \ldots \ldots \ldots$

8.1 Cortical GABA BP $_{\mathrm{ND}}$ distribution. $\ldots \ldots \ldots \ldots \ldots$

8.2 Node connections and distance dependencies. . . . . . . . . . . . . 102 
8.3 Node-specific metrics. . . . . . . . . . . . . . . . . . . 103

8.4 Network modules. . . . . . . . . . . . . . . . . . . . 105

9.1 Overview of analysis. . . . . . . . . . . . . . . . . 115

9.2 Group contrast images. . . . . . . . . . . . . . . . . . 116

9.3 Predicted and actual fMRI responses for $[$ int $>$ ext $] \ldots \ldots \ldots \ldots$

9.4 Model coefficients for $[$ int $>$ ext contrast. . . . . . . . . . . . . . . 119

9.5 Model coefficients for $[$ int $>$ fix $]$ and $[$ ext $>$ fix $] \ldots \ldots \ldots \ldots . \ldots . \ldots 122$

10.1 Overview of study design. . . . . . . . . . . . . . . . . 131

10.2 Relationship between mPFC Glu/entropy and CTQ . . . . . . . . . . 136

10.3 Whole-brain regressions of $[\mathrm{ACA}>\mathrm{Safe}]$ and study measures. . . . . . . 138

10.4 Overview of study results. . . . . . . . . . . . . . . . . 142

A.1 Distribution of FC z-scores. . . . . . . . . . . . . . . 216

A.2 Correlations between FC and mPFC Glu/NAA . . . . . . . . . . 217

A.3 Correlations between FC and track count. . . . . . . . . . . . . . 218

C.1 Linear fits between GM and BP $P_{N D}(A) \ldots \ldots \ldots \ldots 227$

C.2 Linear fits between $\mathrm{GM}$ and $\mathrm{BP} \mathrm{P}_{\mathrm{ND}}(\mathrm{B}) . \ldots \ldots \ldots \ldots . \ldots . \ldots . \ldots 28$

D.1 Group images for contrasts $[$ int $>f i x]$ and $[$ ext $>f i x] \ldots \ldots \ldots . \ldots 230$

E.1 Childhood trauma questionnaire. . . . . . . . . . . . . . 238

E.2 [AUA > Safe] responses with CTQ, Glu/NAA, and entropy regressions. . 239 


\section{List of Tables}

2.1 mPFC FC correlations. . . . . . . . . . . . . . . . 25

3.1 Regions showing correlation with pgACC Glx/Cr. . . . . . . . . 47

5.1 Regions showing change in entropy and SD . . . . . . . . . 71

7.1 ROI volumes, binding potentials, and correlations. . . . . . . . . 91

8.1 Graph small world properties. . . . . . . . . . . . . . . 103

8.2 FMZ-BP $_{\mathrm{ND}}$ network sub-modules. . . . . . . . . . . 107

9.1 MAEs for left and right insula models. . . . . . . . . . . . . . . . . . 119

9.2 Model coefficients for $[$ int $>$ ext] contrast. . . . . . . . . . . 120

10.1 Subject demographics. . . . . . . . . . . . . . . . 128

10.2 Summary of score/RS measure correlations. . . . . . . . . . . . 135

10.3 Results for contrast $[\mathrm{ACA}>\mathrm{Safe}] \ldots \ldots \ldots \ldots \ldots$

10.4 Results for $[\mathrm{ACA}>\mathrm{Safe}]$ vs $\mathrm{CTQ}$ regression. . . . . . . . . . . . 140

10.5 Results for $[\mathrm{ACA}>\mathrm{Safe}]$ vs $\mathrm{mPFC}$ Glu/NAA regression. . . . . . . . . . 140

10.6 Results for $[\mathrm{ACA}>\mathrm{Safe}]$ vs mPFC Ent regression. . . . . . . . . . . 140

A.1 MRS values for mPFC and left insula. . . . . . . . . . . . . . 219

A.2 Regional and biochemical control correlations. . . . . . . . . . 220

B.1 Empathy task signal changes. . . . . . . . . . . . . . . . . 222

B.2 Empathy task deactivations. . . . . . . . . . . . . . . 223

B.3 Regions showing positive connectivity with $\operatorname{pgACC} \ldots \ldots \ldots \ldots . . .224$

B.4 Regions showing positive connectivity with sgACC. . . . . . . . . . 224

B.5 Correlations between task response and $\mathrm{sgACC}$ Glx $/ \mathrm{Cr} . \ldots \ldots \ldots . . . .225$

D.1 Activations for contrast $[$ int $>$ ext $] \ldots \ldots \ldots \ldots \ldots \ldots \ldots \ldots \ldots \ldots$ 
D.2 Activations for contrast $[$ ext $>$ int $] \ldots \ldots \ldots \ldots 232$

D.3 Activations for contrast $[$ int $>$ fix $] \ldots \ldots \ldots \ldots \ldots \ldots \ldots \ldots \ldots$

D.4 Activations for contrast $[\mathrm{ext}>\mathrm{fix}] \ldots \ldots \ldots \ldots . \ldots . \ldots 234$

D.5 Model coefficients for [int $>$ fix] and [ext $>$ fix] responses in left insula regions. 235

D.6 Model coefficients for [int $>$ fix] and [ext $>$ fix] responses in right insula regions. 236

E.1 Activations for $[\mathrm{AUA}>\mathrm{Safe}]$ contrast. . . . . . . . . . . . 240

E.2 Cluster locations for [ACA vs Safe] vs CTQ regression. . . . . . . . . . . 241

E.3 Cluster locations for [AUA vs Safe] vs entropy regression . . . . . . . . . 241 


\title{
List of abbreviations
}

\author{
$\mathrm{ACC}$ - anterior cingulate cortex \\ AC-PC - anterior commissure to posterior commissure \\ BOLD - blood oxygenation-level dependent \\ $\mathrm{BP}_{\mathrm{ND}}$ - binding potential \\ Cr - creatine
}

CRLB - Cramér-Rao lower bounds

CSF - cerebrospinal fluid

CTQ - childhood trauma questionnaire

dACC - dorsal anterior cingulate cortex

DMN - default-mode network

DMT - dorso-medial thalamus

DTI - diffusion tensor imaging

EC - eyes closed

EEG - electroencephalography

ELS - early-life stress

EO - eyes open

${ }^{18} \mathrm{~F}$ - fluorine- 18 
FA - fractional anisotropy

fALFF - fractional amplitude of low-frequency fluctuations

$\mathrm{FC}$ - functional connectivity

FDR - false discovery rate

fMRI - functional magnetic resonance imaging

FMZ - flumazenil

FoV - field of view

FWE - family-wise error

FWHM - full-width half-maximum

GABA - $\gamma$-aminobutyric acid

Glu - glutamate

Gln - glutamine

Glx - glutamate+glutamine

GLM - general linear model

GM - grey matter

ITI - inter-trial interval

LI - left insula

MDD - major depressive disorder

mPFC - medial prefrontal cortex

MRI - magnetic resonance imaging

MRS - magnetic resonance spectroscopy 
NAA - N-acetylaspartate

NAc - nucleus accumbens

NBR - negative BOLD response

N.MR - nuclear magnetic resonance

PAG - periaqueductal grey

PBR - positive BOLD response

PCC - postcrior cingulate cortex

PET - positron emission tomography

PFC - prefrontal cortex

pgACC - perigenual anterior cingulate cortex

PPI - psychophysiological interaction

ROI - region of interest

$\mathrm{RF}$ - radiofrequency

SD - standard deviation

sgACC - supragenual anterior cingulate cortex

SNR - signal-to-noise ratio

$\mathrm{T}_{\mathrm{E}}$ - excitation time

$\mathrm{T}_{\mathrm{R}}$ - repetition time

VBM - voxel-base morphometry

VOI - volume of interest

WM - white matter 


\section{Chapter 1}

\section{General introduction}

\subsection{The brain as a dynamic network}

For much of the past 150 years, the main paradigm for studying the brain has been one of localisation. Under this conception, the brain is seen as a collection of modular regions onto which distinct psychological processes can be mapped (Catani et al., 2012). More recently, however, a shift towards investigating the brain in terms of networks has taken place (Bressler and Menon, 2010). Instead of processes being mapped to particular individual locations, this network-based conception holds that their substrates are distributed groups of inter-connected regions throughout the brain (Bartolomeo, 2011, Catani et al., 2012). For example, a cross-species core network in aversive processing has been described (Hayes and Northoff, 2011), whilst functional imaging has been used to identify similar core networks for, amongst other processes, emotion (Lindquist et al., 2012) and empathy (Fan et al., 2011) in humans. Of course, it should be noted that the lived brain must exist in a dynamic admixture of these possible process-related network states.

The neuroimaging work that has played a large role in describing these networks in humans has mainly exploited carefully designed tasks in order to target particular psychological processes. Using such an approach, regions (and subsequently networks) are identified by comparing the brain in one process-state to another, isolating the differences. An example of this would be the mapping of an aversion network, where tasks involving the comparison of, for example, painful to non-painful tactile stimuli have been used (Hayes and Northoff, 2012). At the same time as such stimulus-oriented research was being undertaken, it became increasingly obvious that the brain displayed a high level of activity that was independent of any particular stimulus (Northoff et al., 2010a, Raichle, 2009). First described in this 


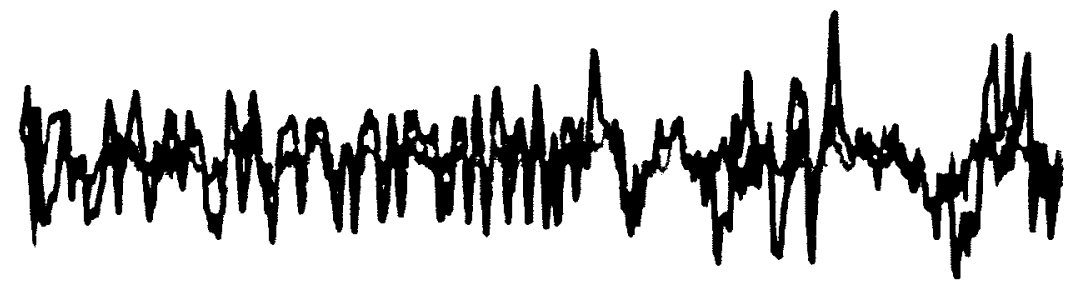

Figure 1.1: Example timecourses and the correlational functional connectivity between them.

context was is set of regions. termed the default-mode network (D.M), whose activity levels are higher when a subject is not participating in any specific task. but are instead lying at rest (Raichle et al., 2001)(leading to them being described, arguably misloadingly (Golland et al., 2007, Sadaghiani et al., 2010), as "resting-state" networks). These DMN regions include the posterior cingulate, precuneus, and medial prefrontal cortex.

Investigations into this task-independent activity revealed spontaneous fluctuations at low frequencies of less than $0.1 \mathrm{~Hz}$ that are correlated across the network. Such ongoing fluctuations are not limited to the DMN, however, and similar properties can be seen in other anatomical subdivisions of the brain that map broadly with functions (such as a somatosensory network or visual network)(Smith et al., 2009). Importantly: this organisational property of the brain appears to be conserved to varying degrees across states of consciousness (Heine et al., 2012), and across species (Hutchison and Everling, 2012), suggesting that it is in some way fundanental. Also important to note. however, is that these large-scale networks can be further divided into more fine-grained and apparently functionally relevant components (Margulies et al., 2009, Zhang and Li, 2012) and that the delineations between intrinsic activity networks are not rigid (Cohen et al., 2008. Gonzalez-Castillo et al., 2012, Sridharan et al., 2008).

These temporal correlations in activity fluctuations (Figure 1.1) have been termed functional connectivity and must be distinguished from effective comnectivity. where a causative link between two regions can be established (Friston et al., 1995). To begin to bridge this gap between correlational and causative networks, the large-scale patterns of ongoing activity in the brain have been compared to information about structural connectivity between 
regions. Such studies have indeed shown that the the networks arising out of intrinsic activity fluctuations map well to patterns of axonal links between regions (Greicius et al., 2009, Honey et al., 2009, Skudlarski et al., 2010). However, the correlation between the two is not perfect, something that is in line with a mutability of functional inter-relations between network regions over time and in response to changing psychological contexts (Honey et al., 2009, Sadaghiani et al., 2010).

Do these ongoing fluctuations have any impact upon our experience, however? Switching from a large-scale network view, ongoing activity has been shown to have direct effects upon stimulus-induced activity within particular regions. One of the first demonstrations of this was that made by Arieli and colleagues (Arieli et al., 1996), in which ongoing activity fluctuations in cat visual cortex activity determined the size of the response in that region to a stimulus. In humans, more complex interactions between ongoing activity fluctuations and stimuli have been seen, with, for example the image perceived in optical illusions and the perception or not of an at-threshold auditory stimulus being predicted by the prestimulus activity level in the fusiform face area and auditory cortex, respectively (Boly et al., 2008, Hesselmann et al., 2008, Sadaghiani et al., 2010). In addition, differences in pre-stimulus neural activity strengths and coherences at various frequencies, as measured across the scalp, have similar predictive power of perceptual or behavioural responses to particular stimuli (Busch et al., 2009, Linkenkaer-Hansen et al., 2004). Such findings thus add to those from large-scale network fluctuations to underline the potential importance of ongoing activity to cognition.

\subsection{Questions}

From the described background, the question arises as to the potential mediators of the dissociation of structural and functional network properties and of ongoing activity itself. It may be that the differences between structural and functional network measures are down to methodological limitations or that they represent true divergence between structure and function. At the same time, it is possible that the measuring of static (within short timescales) structural properties along with functional activity does not capture fully the necessary mediating link between the two, giving rise to the apparent disconnect. In the case of the brain, the most important links between pure structure (i.e., the gross layout 
of neurons and connections) and the functional activity arising from it are the transmitters and receptors that are the effectors of activity. At present few studies in humans have investigated such biochemical links between structural brain networks and the functional activity that they support (Northoff et al., 2007, Kapogiannis et al., 2012). It is the making of initial such links that is the target of the experiments described here. At the same time, whilst some work has begun to describe the biochemical underpinnings of ongoing activity in humans (Donahue et al., 2010, Muthukumaraswamy et al., 2009, Northoff et al., 2007), this remains an under-investigated area with many outstanding questions.

In order to approach these points, this work presents a set of multimodal imaging studies in humans. Magnetic resonance imaging (MRI) was combined with positron emission tomography (PET) and electroencephalography (EEG) in order to take advantage of the different strengths of the different techniques. MRI allows for excellent spatial localisation of functional changes, as well as allowing some biochemical measurements and detailed analysis of the structural pathways within the brain. Rather than information about spatial properties, EEG provides greatest insight into the temporal properties of brain activity, giving resolution of brain responses at the millisecond level. It will be used here to investigate the temporal structure of ongoing activity. PET was used to measure neural receptors in order to investigate biochemical underpinnings of the brain's intrinsic activity. (Details of the methods used are given at the relevant point below.)

The work is presented here as a series of stand-alone papers that have been published or have been submitted for publication in peer-reviewed journals. Each paper can be taken independently from the others; however, to outline the continuity between them and to presents additional details or analyses, additional introductory or concluding sections have been added (where necessary). Where the paper has supplementary information that may be referred to, this is include in a series of appendices at the end of the work and is referenced within the paper's text. Note that whilst each chapter is hypothesis driven, the whole work is bound by the themes outlined above rather than an overarching hypothesis. The work is thus intended to contribute to the generation of future hypotheses, as outlined in each chapter's discussion section and in the final discussion in Chapter 11. The chapters included can be summarised as follows:

- Chapter 2 consists of an analysis linking biochemical measures (specifically of glutamate) from the medial prefrontal cortex (mPFC) with functional and structural 
connectivity between that region and a set of subcortical regions during rest.

- Chapter 3 advances the findings of Chapter 2 by investigating the link between mPFC biochemistry and functional connectivity with that region during a specific task (as opposed to during rest).

- Chapter 4 and 5 provide complementary analyses of the role of glutamate in activity dynamics within the mPFC. Chapter 4 used a "classic" general linear model approach, whilst Chapter 5 employs more complex linear and non-linear measures.

- Chapter 6 and 7 outline relevant background for positron emission tomography (PET) using the $\mathrm{GABA}_{\mathrm{A}}$ receptor ligand flumazenil. A preliminary analysis for selecting reference regions is presented, followed by an analysis clarifying the utility of flumazenil imaging for cross-subject receptor comparisons. This analysis provides evidence for the validity of the analysis presented in Chapter 8 .

- Chapter 8 uses graph-theoretical measures to describe GABAA $_{A}$ receptor networks in the cortex and discusses these as a potential bridge between network descriptions at the structural and functional level.

- Chapter 9 combines an intero- and exteroception fMRI task with diffusion tensor imaging to investigate how structural networks relate to functional responses.

- Chapter 10 investigates the effect of early-life stress on brain biochemistry and intrinsic activity and how changes in these can affect individual responses to aversive stimuli in adulthood.

- Finally, Chapter 11 provides an overview of the work, along with general conclusions and some suggestions for future research.

Taken together, the work thus aims to approach the brain from an network perspective (Catani et al., 2012) in order to provide additional understanding of such a view of the brain. Centrally, it will do this by providing new information about brain networks at a biochemical level, something that is missing from the current human literature. In addition, it will build on existing knowledge of brain networks at the structural and functional level by combining these two domains in different task contexts. A final underlying aim is to 
provide background knowledge for the study of psychiatric disorders. To this end, findings in each chapter shall be discussed in their potential clinical context.

\subsection{Methodological background}

As described, a number of imaging modalities were employed in this work. In the first group of papers, different MRI scan types are used, along with EEG. A brief, basic overview of these approaches is given here. Relevant background to PET imaging is given in Chapter 6 prior to the introduction of analyses involving such data.

\section{Principles of MRI}

The fundamental physical property on which MRI is built is known as the spin of an atomic nucleus. Taking hydrogen $-{ }^{1} \mathrm{H}-$ as an example, the spin is the rotational motion of the proton in the nucleus around an axis. As the proton rotates, its positive charge means that an electric current is produced on its surface which, as with currents in macroscopic systems, creates a magnetic source that is aligned with the axis of rotation. When this magnetic source is placed into a magnetic field that is not aligned with the axis of rotation, a force is created (to illustrate this we can think of the force that one can feel whilst holding a magnet close to another). The strength of this force, which is proportional to the strength of the magnetic source, is known as the magnetic moment. At the same time as producing a magnetic source, the rotation of the proton also results in a second property of the proton, known as angular momentum, in a manner directly analogous to any other rotating mass, whether it be a bicycle wheel or a planet. With both an angular momentum and magnetic

moment, hydrogen protons are said to have the property of nuclear magnetic resonance (NMR) - a property shared with all other nuclei that have an odd atomic mass.

When located in a static magnetic field, such as that in an MRI scanner, these spinning nuclei can align either parallel or anti-parallel to the longitudinal axis of the field. Those that are aligned against the axis have more energy that those that are parallel to it, meaning that when they flip to being aligned, they release energy and vice versa. Under normal conditions, the numbers of nuclei aligned with the field is slightly higher than those anti-parallel; 
this equilibrium alters, however, as energy is taken or added to the system, increasing or decreasing the number of nuclei anti-parallel to the field and so decreasing or increasing the net magnetisation. In an MRI scanner energy is added to the system by means of a radiofrequency $(R F)$ pulse which knocks a proportion of the nuclei out of alignment with the axis of the field - a high-energy state. When this RF pulse is then turned off, these nuclei return to lying along the axis of the field, releasing photons of energy in the process. It is these photons that are detected by the MRI scanner, their different frequencies and decay times giving information about the surroundings from which they originated. This information is in turn processed in order to produce the particular MRI image that is being created.

\section{Magnetic resonance spectroscopy}

Early in the development of MRI, it was established that the magnetic resonance properties of a nucleus are dependant upon its chemical environment (Proctor, 1950). This discovery provided the ground for the technique of magnetic resonance spectroscopy (MRS). As described, in the scanner's magnetic field nuclei fall into alignment. A radio pulse can then be used to excite them into a position perpendicular to the field, from which they will then relax again once the radio pulse has stopped. It is differences in this relaxation caused by the particular chemical environment that are detected in MRS - the hydrogen nuclei in water molecules $\left(\mathrm{H}_{2} \mathrm{O}\right)$, for instance, will have different relaxation properties to those that are bound in a more complex molecule such as choline $\left(\mathrm{C}_{5} \mathrm{H}_{14} \mathrm{NO}\right)$. By repeated measures of the relaxation signals from within a region of interest it is possible to estimate with a high degree of accuracy the concentrations of particular molecules within that region(Castillo et al., 1996). In a typical human imaging study this process takes between 10 and 15 minutes and gives information for a region of interest with a volume of around 3 $\mathrm{cm}^{3}$. The set of molecules that can be discriminated is limited, however, but includes the neurotransmitters glutamate and $\gamma$-aminobutyric acid (GABA) (Henry, 2010), making the

technique potentially valuable for studying the functional role of these substances in the human brain (Northoff et al., 2007, Horn et al., 2010). 


\section{The BOLD response}

The most common approach for measuring neural activity through MRI uses what is known as blood oxygenation-level dependent (BOLD) contrast. This technique follows a not dissimilar approach to MRS in that it takes advantage of a change in a substance's magnetic properties according to it's chemical context. Specifically, BOLD contrast results from the fact that haemoglobin, the molecule that transports oxygen in the blood, has no magnetic moment when it is carrying an oxygen molecule but does have one when no oxygen molecule is present. This means that deoxygenated haemoglobin will interact more with the scanner's magnetic field, in turn meaning that the ${ }^{1} \mathrm{H}$ nuclei (generally) in its vicinity will produce slightly different MRI signals from those that are not in the vicinity of deoxygenated haemoglobin.

Of course, knowing that blood is oxygenated or deoxygenated does not give one an obvious link to information about neurons firing; indeed, the link remains debated, although is becoming increasingly clear (Buxton, 2010, Kim and Ogawa, 2012). In brief, the firing of neurons requires energy, which, in the brain, comes from glucose being metabolised in the presence oxygen (usually). Both glucose and oxygen are brought to the neurons in the blood supply, meaning that when a region has an increase in firing, it requires an increased amount of new blood in order to provide these. As this new blood is brought in to the region, it displaces old, deoxygenated blood. This movement of deoxygenated blood then increases the magnetic interaction of the regions that it moves into, whilst the new, oxygenated, blood reduces the interaction of the active region. It is this change in magnetic interaction that is detected in a functional BOLD contrast image. As the BOLD response is one of blood movement, it occurs quite slowly and lasts for a prolonged period. In general, there is a lag of approximately a second between the firing event and the beginning of the BOLD response, with its peak not occurring until between four and six seconds post-stimulus. This means that BOLD imaging does not have a particularly good temporal resolution. The changes in blood flow that are measured occur in the micro-vascularture, however, meaning that a spacial resolution in the millimetre range is possible (Logothetis, 2002). 


\section{Diffusion tensor imaging}

Axons wrapped in myelin sheath form the major connections between different regions of the brain. These axons, generally referred to as white matter, can be thought of as a dense network of pipes running in bundles through which neural signals are transmitted. As pipes, it is easier for liquids to run along them than it is for the liquid to seep out through the walls. It is this property of axons that diffusion tensor imaging (DTI) exploits in order to create images that reveal the directions in which they travel. In essence, the magnetic field in the scanner is used to measure the movement of water molecules within the brain as a result of their natural Brownian motion - those molecules that are within white matter will move most easily along the direction of the axon, whilst those in other tissues will be constrained by their surrounding cell walls. When sent, the magnetic field pulses interact with the NMR properties of the nuclei in a manner that can be corrected by a reversal of the field. However, those nuclei that have been able to move between field reversals will not be returned to exactly the same state, as their change in position alters the way that the returning field will interact with them, in turn affecting the signals that are received by the scanner's detectors. By taking many measurements of these changes from different directions, it is possible to build up a three-dimensional model - a tensor of the diffusion properties within any particular voxel within the brain, where the model is elongated approximately along the line that the axon travels in.

\section{EEG}

The firing of neurons represents a movement of ions in and out of the cell, producing a small electrical potential. Although the potential produced by a single neuron is minuscule, the synchronised firing of groups of neurons produces enough of a combined electrical potential to be detected by sensitive instrumentation. In electroencephalography (EEG), a net of electrodes placed against the scalp are used to pick-up these neuronal electrical potentials in humans in vivo. The number of electrodes used can range from two up to 128 and beyond, depending on the application they are to be used for.

By directly detecting the electrical potentials produced by neural activity, EEG can have an extremely high temporal resolution, in the millisecond range. However, because the 
electrical potentials are propagating in all directions through the head and are interacting with each other as they do so, it is not possible for the location of a particular source to be determined exactly. This means that EEG has a poor spatial resolution, although techniques do exist that allow good approximations to be made. 


\section{Chapter 2}

\section{Glutamate in the mPFC predicts resting-state cortical-subcortical functional connectivity}

\subsection{Abstract}

Communication between cortical and subcortical regions is integral to a wide range of psychological processes and has been implicated in a number of psychiatric conditions. Studies in animals have provided insight into the biochemical and connectivity processes underlying such communication. However, to date no experiments that link these factors in humans in vivo have been carried out. To investigate the role of glutamate in individual differences in communication between the cortex - specifically the medial prefrontal cortex ( $\mathrm{mPFC}$ ) - and subcortical regions in humans, a combination of resting-state $\mathrm{fMRI}$, DTI and MRS was performed. The subcortical target regions were the nucleus accumbens (NAc), dorsomedial thalamus (DMT), and periaqueductal grey (PAG). It was found that functional connectivity between the $\mathrm{MPFC}$ and each of the NAc and DMT was positively correlated with $\mathrm{MPFC}$ glutamate concentrations, whilst functional connectivity between the $\mathrm{mPFC}$ and PAG was negatively correlated with glutamate concentration. The correlations involving $\mathrm{mPFC}$ glutamate and $\mathrm{FC}$ between the $\mathrm{mPFC}$ and each of the DMT and PAG were mirrored by correlations with structural connectivity, providing evidence that the glutamatergic relationship may, in part, be due to direct connectivity. These results are in agreement with existing results from animal studies and may have relevance for MDD and schizophrenia. 


\subsection{Introduction}

Effective communication between cortical and subcortical regions is likely essential for all psychological functions, as well as for the intrinsic brain networks seen at rest. For example, recent reports have underscored the importance of such communication in higher order emotional functioning as well as in more fundamental reward and aversion-related processing (Hayes and Northoff, 2011, Liu et al., 2011, Ochsner and Gross, 2005). Detailed knowledge of the systems involved in such cortical-subcortical communication has been obtained through many years of non-human animal studies which have provided an increasing understanding of the networks and numerous neurotransmitters involved, highlighting, for example, the roles of the medial prefrontal cortex (mPFC) and transmitters such as glutamate (Del Arco and Mora, 2009, Cummings, 1995, Floresco et al., 2009, Tekin and Cummings, 2002).

Given the obvious experimental challenges, in vivo studies of cortical-subcortical connections in humans have, in contrast, been less common. However, advances in imaging technology now make it possible for studies to be carried out that link structure, function, and biochemistry (Duncan et al., 2011, Hao et al., 2011, Honey et al., 2009). In addition to advancing our basic understanding of the brain, such research may be of particular interest as changes in cortical-subcortical communication have been proposed to underlie a variety of psychiatric disorders, including depression and schizophrenia (Del Arco and Mora, 2009, Kühn and Gallinat, 2011, Northoff et al., 2011). In the latter context, glutamatergic communication presents a promising target as this transmitter has been implicated in each of these disorders (Hashimoto, 2009, Schmidt and Pierce, 2010, Stone et al., 2012).

The current study aimed to undertake a preliminary investigation of the biochemical underpinnings of inter-individual differences (Kanai and Rees, 2011) in resting-state corticalsubcortical communication in humans, focusing on glutamate in the mPFC. Using a combination of resting-state MRI, diffusion-tensor imaging (DTI), and magnetic resonance spectroscopy (MRS), functional and structural connectivity between the mPFC (centred on the perigenual anterior cingulate; $\mathrm{pgACC}$ ) and three selected subcortical regions were measured. These regions were the nucleus accumbens (NAc), dorsomedial thalamus (DMT), and periaqueductal grey (PAG). Given the exploratory nature of the investigation, this small number of target regions was used to minimise the problem of multiple comparisons. 
The target regions chosen were selected, firstly, due to their known anatomical connections with the $\mathrm{mPFC}$, and secondly due to these connections being known to involve glutamate to some degree (Butler et al., 2011, Coutinho and Menescal-de Oliveira, 2010, Dawson et al., 2011, Groenewegen et al., 1999, Jodo et al., 2010, Price, 2007). In addition, the regions were selected as they are components of what has been termed the core and para-core limbic systems that underlie fundamental stimulus processing (Morgane et al., 2005, Panksepp, 1998).

MRS was used to measure resting-state glutamate and glutamine concentrations within the mPFC. Glutamatergic measures were then correlated with the degree of resting-state functional connectivity (FC) between the mPFC and each of the target regions. DTI was used to establish if the FC observed was related to structural connectivity, and thus direct inter-regional communication. In order to provide some degree of specificity to any observed relationship between $\mathrm{mPFC}$ glutamatergic measures and $\mathrm{FC}$, glutamatergic MRS measures from the left insula were used as regional control values.

Although the study was mainly exploratory, it was hypothesised, given the known glutamatergic connections between the regions and that this transmitter has been implicated in inter-regional communication in vivo in humans (Duncan et al., 2011), that glutamate levels in the mPFC would be positively related to resting-state functional connectivity. As the relationship between functional and structural connectivity is complex (Aslan et al., 2011, Honey et al., 2009), no hypotheses were proposed in this regard.

\subsection{Materials and methods}

\subsubsection{Participants}

Twenty-eight healthy participants were scanned using fMRI and MRS at two different MRI centres (fMRI - Montreal Neurological Institute, McGill University; MRS - Unité de neuroimagerie fonctionelle, Université de Montréal). Siemens 3 Tesla Trio MRI scanners were used at both locations. The data from six subjects were rejected due to excessive head movement during the fMRI scan, four subjects due to deviance from the task, and from five subjects due to poor quality MRS data. The analyses were carried out on the rest of 
the data from 13 subjects ( 5 females; mean age $=22.1$ years, range: $18-32$ years). The mean time between scans for the subjects to be included was 3.6 days (range: 1-10 days). Subjects were screened for psychiatric or neurological disorders, recreational drug use, and depression, the latter using the Beck Depression Inventory-II with a cut-off score of four (Beck et al., 1996). All subjects gave their written informed consent and were compensated financially for their participation. Approval for the study was obtained from the ethics committees at both McGill University and the Université de Montréal.

\subsubsection{MRS}

Single voxcl edited $1 \mathrm{H}$ MR spectra were acquired using the MEGA-PRESS method (Marjanska et al., 2007, Mescher et al., 1998) with a body coil transmit and 12-channel receive headcoil. Using a high resolution $T_{1}$-weighted anatomical image (MPRAGE; FoV $=205 \mathrm{x}$ $205 \mathrm{~mm}^{2}$; spatial resolution $=1 \times 1 \times 1 \mathrm{~mm}^{3} ; \mathrm{T}_{\mathrm{E}}=3.02 \mathrm{~ms} ; \mathrm{T}_{\mathrm{R}}=2000 \mathrm{~ms}$; flip angle $=$ $5^{\circ}$ ), volumes of interest (VOI) were located in the $\mathrm{mPFC}$ and the left insula (Figure 2.1). In order to achieve consistent VOI positioning, placement was done by the same investigator for all subjects according to easily identifiable anatomical landmarks: mPFC VOIs $(48 \times 21$ x $21 \mathrm{~mm}^{3}$ ) were placed anterior to the genu of the corpus callosum, parallel to the AC-PC plane; left insula VOIs $\left(23 \times 48 \times 27 \mathrm{~mm}^{3}\right)$ were aligned with the line of the insula cortex in an anterior-posterior direction with the most anterior edge of the VOI aligned to the anterior limit of the insula.

First- and second-order shim terms were adjusted using FASTMAP with echo-planar imaging readout (Gruetter and Tkác, 2000). MRS data were acquired using a MEGA-PRESS sequence (Mescher et al., 1996, 1998) with double-banded pulses used to simultaneously suppress water signal and edit the gamma-CH2 resonance of $\mathrm{GABA}$ at $3 \mathrm{ppm}$. Additional water suppression using variable power with optimised relaxation delays (VAPOR) and outer volume suppression (OVS) techniques (Tkác et al., 1999) was optimised for the human 3 Tesla system and incorporated prior to MEGA-PRESS. The final spectra were obtained by subtracting the signals from alternate scans with the selective double-banded pulse applied at $4.7 \mathrm{ppm}$ and $7.5 \mathrm{ppm}$ ('EDIT OFF') and the selective double-banded pulse applied at $1.9 \mathrm{ppm}$ and $4.7 \mathrm{ppm}$ ('EDIT ON'). MEGA-PRESS data were acquired in four interleaved blocks of 32 ('EDIT OFF', 'EDIT ON') scans each with frequency adjustment

between each block. FIDs were stored separately in memory for individual frequency and 


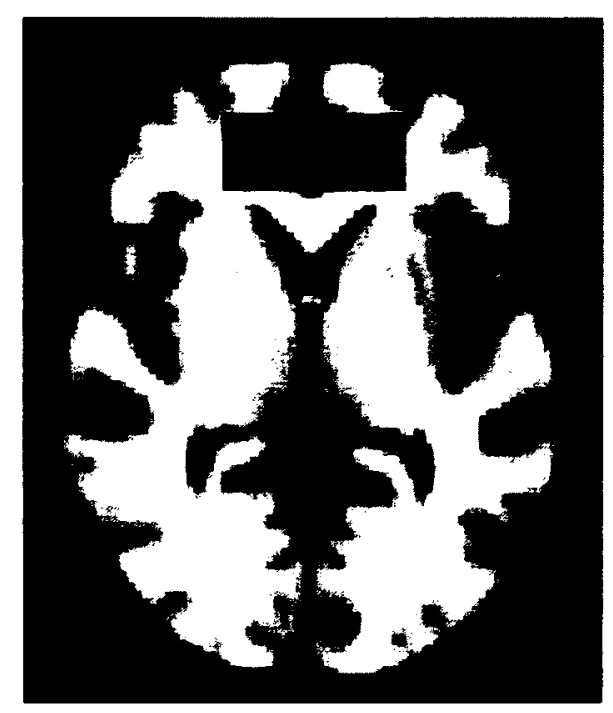

Figure 2.1: mPFC (blue) and left insula (red) MRS voxel locations shown on the MNI template.

phase correction using the tCr signal at 3.03 ppm. as well as correction for residual eddycurrent using unsuppressed water signal obtained from the same voxel.

Difference spectra were analysed using LCModel 6.2-1A (Provencher, 1993. 2001) using the basis set which included an experimentally measured metabolite-nulled macromolecular spectrum (average from 10 subjects) and the experiment ally measured spectra from $100 \mathrm{mM}$ phantoms of $\mathrm{N}$-acetylaspartate ( $\mathrm{NAA}$ ), $\gamma$-aminobutyric acid (GABA), glutamate (Glu). and glutamine ( $\mathrm{Gln}$ ) with $\mathrm{pH}$ adjusted to 7.2 and at $37^{\circ} \mathrm{C}$. The LCMIodel fitting was performed over the spectral range from 0.5 to $4.0 \mathrm{ppm}$. No baseline correction, zero-filling, or apodization functions were applied to the in vivo data prior to LCModel analysis.

Only results with the Cramer-Rao lower bounds (CRLB) below 20\% were included in the analysis. Concentrations with CRLB $=20 \%$ were classified as not detected. Although the estimated correlation cocfficients, derived from a standard least-squares variance-covariance matrix of LCModel analysis, indicated a strong negative correlation between Glu and Gln, both Glu and Gln were reliably quantified in all subjects. LCModel quantification of the representative spectrum is shown in Figure 2.2.

The mPFC, centred on the perigenual anterior cingulate cortex (pgACC), was the target 


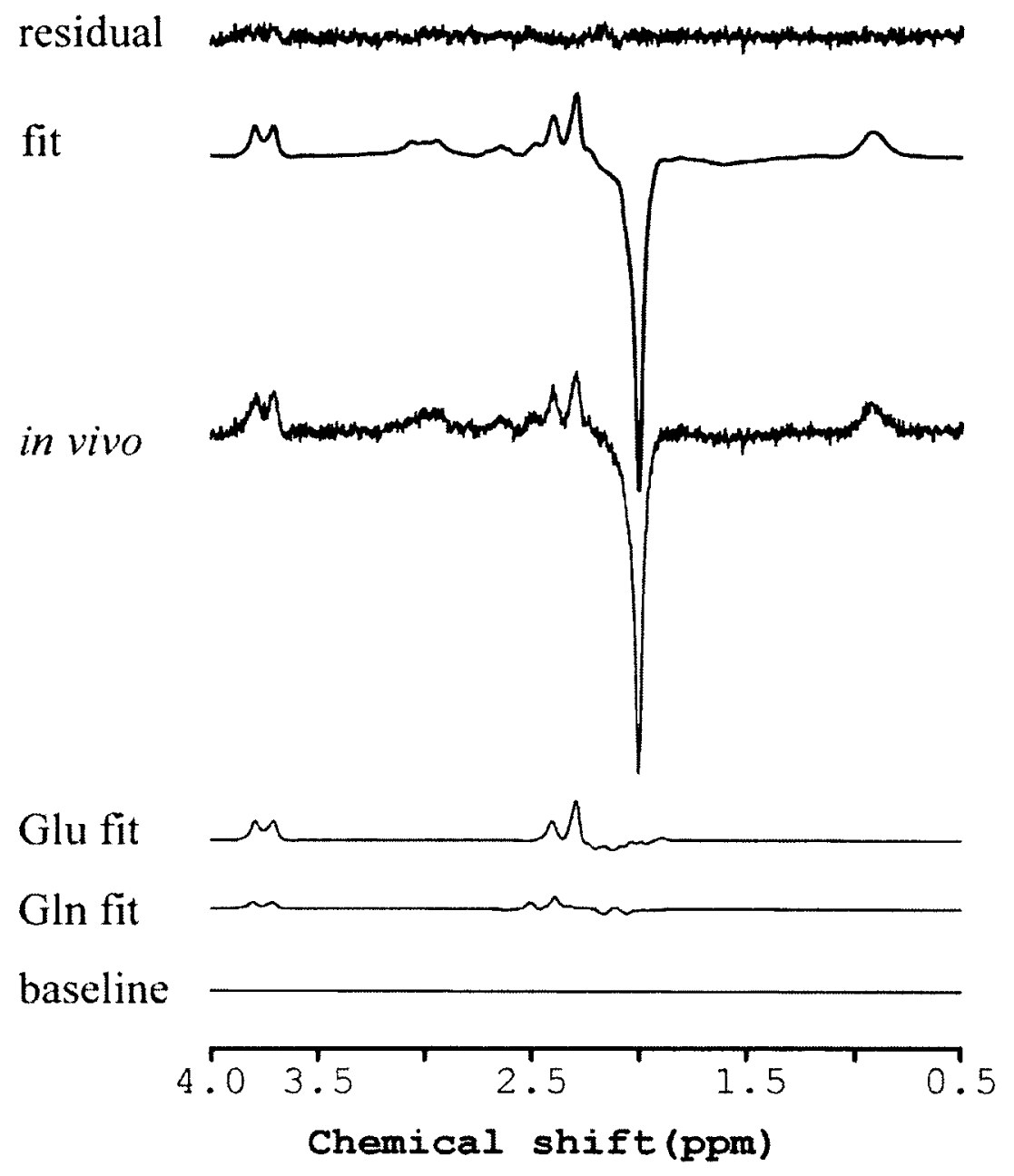

Figure 2.2: LCModel quantification of the representative ${ }^{1} \mathrm{H}$ NMR spectrum obtained using MEGAPRESS sequence from a $48 \times 21 \times 21 \mathrm{~mm}^{3}$ voxel placed in the $\mathrm{mPFC}$ region of the brain. The contribution of Glu and Gln are shown. 
region for the study and the left insula was used as a regional specificity control. The first step in each analysis was to use the combined Glu $+\mathrm{Gln}(\mathrm{Glx})$ concentrations, as a ratio to $\mathrm{NAA}$, for correlation with functional connectivity (Glx/NAA). In a second step, functional connectivity was correlated with the individual values for Glu/NAA and Gln/NAA.

\subsubsection{ROls}

The MPFC MRS region was used as the seed ROI. Left and right NAc ROIs were defined as the NAc region from the Harvard-Oxford subcortical atlas (probability 0.5 ) (http://ww . cma.mgh.harvard.edu/fsl_atlas.html). Spherical target ROIs with a radius of $4 \mathrm{~mm}$ were made in the DMT and PAG bilaterally in standard space (MNI). Coordinates for the DMT ROIs (left $=12-188$; right $=-10-188$ ) were obtained by identifying the thalamus on the mean group anatomical image using the Harvard-Oxford atlas and then visually centring the spheres within the dorso-medial portion. Coordinates for the PAG ROIs (left $=-4289$; right $=4-28-6$ ) were adapted from the literature (Kong et al., 2010). See Figure 2.3 for ROI locations.

\subsection{4 $\mathrm{fMRI}$}

Functional EPI scans were acquired using a body coil transmit and 32-channel receive headcoil. Forty-seven slices aligned at $-30^{\circ}$ from the AC-PC plane and covering the whole brain were acquired per volume, with a total of 467 volumes being acquired (1060 s) over the task run ( $\mathrm{FoV}=205 \times 205 \mathrm{~mm}^{2}$; spatial resolution $=3.2 \times 3.2 \times 3.2 \mathrm{~mm}^{3} ; \mathrm{T}_{\mathrm{E}}=25 \mathrm{~ms}$; $\mathrm{T}_{\mathrm{R}}=2270 \mathrm{~ms}$; flip angle $\left.=90^{\circ}\right)$. The first five volumes were discarded. A high-resolution $\mathrm{T}_{1}$-weighted anatomical image was also acquired (MPRAGE; FoV $=205 \times 205 \mathrm{~mm}^{2}$; spatial resolution $=1 \times 1 \times 1 \mathrm{~mm}^{3} ; \mathrm{T}_{\mathrm{E}}=3.02 \mathrm{~ms} ; \mathrm{T}_{\mathrm{R}}=2000 \mathrm{~ms} ;$ flip angle $\left.=5^{\circ}\right)$.

The session consisted of two long eyes-open (EO) and two long eyes-closed (EC) periods (2 x $120 \mathrm{~s}, 212$ volumes total, alternating and counterbalanced across subjects; Figure 2.4a), that followed on from a sequence of short EO and EC blocks (255 volumes), the data for which were not analysed here. EC conditions were indicated by a single short tone and EO by a short double tone (100 ms and $2 \times 100 \mathrm{~ms}$ ). Small EO and EC icons were also displayed 


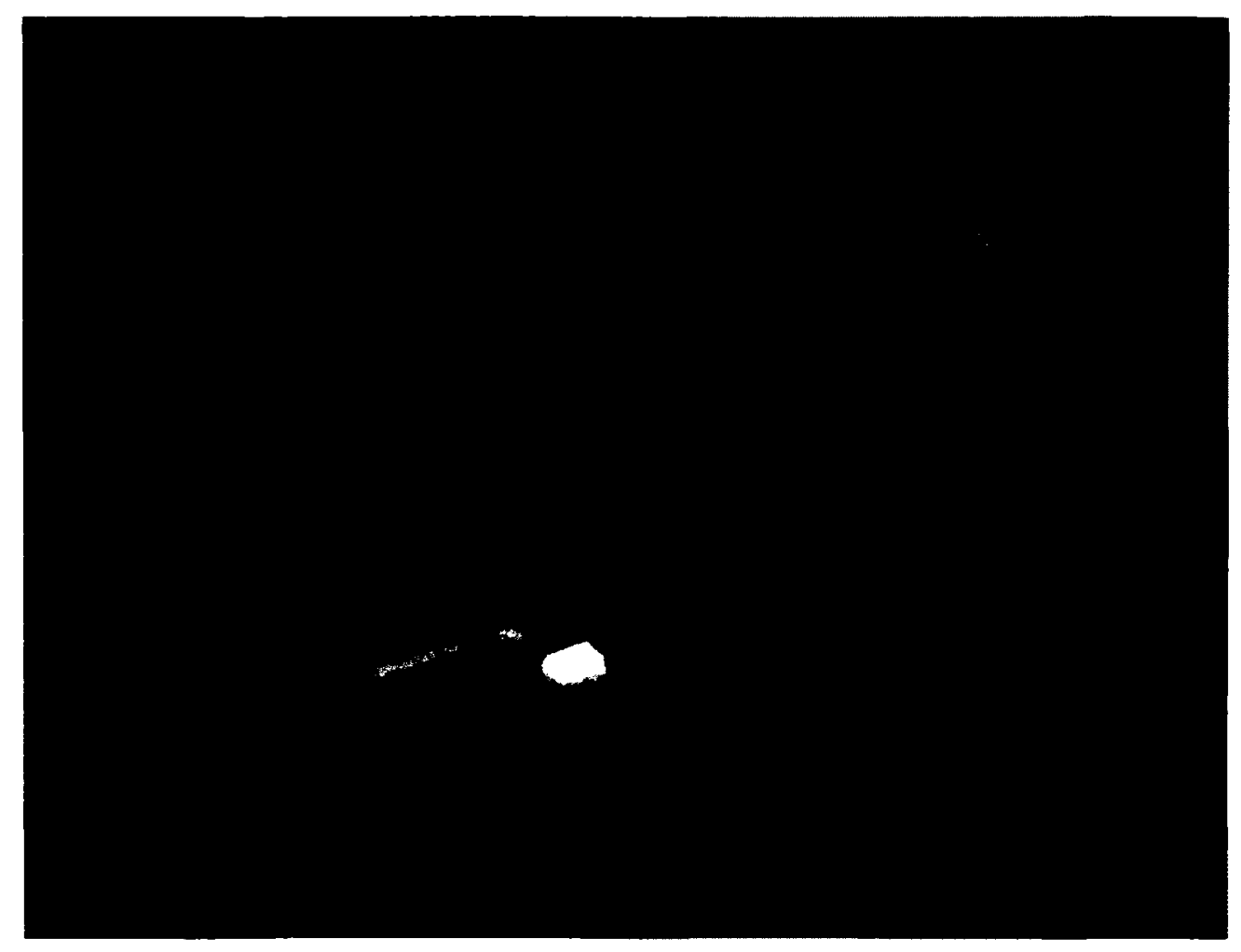

Figure 2.3: Location of ROIs, displayed on group mean anatomical image. Orange - mPFC, yellow NAc, blue DMT, red PAG.

to inform subjects of the current condition should they be confused. Subjects were observed using a simple camera setup to ensure that they followed the task instructions.

The processing of all AMRI data was carricd out with the FSL suite of tools (Smith ct al., 2004. Woolrich et al. 2009). Functional images were corrected for head movement, brainextracted, high-pass filtered with a $100 \mathrm{~s}$ cut-off, and smoothed with a $6 \mathrm{~mm}$ at FWHM Gaussian kemel. Functional data was then variance normalised by dividing each roxel by its standard deviation over time. Anatomical images were brain-extracted prior to segmentation into white matter (WM), grey matter (GM), and cerebrospinal fluid (CSF) mays using FAST (Zhang et al., 2001). Linear aligmments between the functional images and the MNI template were calculated via the high-resolution anatomical image.

Masks corresponding to the mPFC and left insula MRS boxes were applied to the individual 
G.M maps, thresholded at 0.95 , to produce subject specific masks of GM within the MRS boxes (MRS-GM). The proportion of grey matter within the MRS box was included in all group-level analyses as a confounding variable. Following binarisation, these masks were transformed into individual functional space and used as ROIs for the functional connectivity analyses. Mean timecourses were extracted from within the MRS-GM ROIs (from the unsmoothed pre-processed data). These were then mean-centred, variance normalised, and split into the EO and EC conditions (Figure 2.4b).

Segmented WM and CSF maps were made into binary mask images using a threshold of 0.99 and then eroded by one voxel to ensure that there was no overlap between the masks and GM. These WM and CSF ROIs were transformed into functional space and the first eigenvariate of the timecourse in each ROI extracted for each subject (from the unsmoothed pre-processed data). These CSF and WM timecourses were included in the functional connectivity model as nuisance regressors along with the six head movement parameters obtained during pre-processing. These regressors were included to minimise the effect of head movement and physiological and other noise sources on the functional connectivity calculations.

A model consisting of the $\mathrm{EO}$ and $\mathrm{EC}$ timeseries from the mPFC (Figure 2.4b), along with the eight nuisance regressors, was constructed and entered into a multiple regression analysis with the variance normalised functional data (Greicius et al., 2003, Jolles et al., 2011, Margulies et al., 2007). In this way, functional connectivity during the EO and EC condition was determined, along with the difference in connectivity between these two states $(\mathrm{EO}>\mathrm{EC})$. As age has been observed to affect functional connectivity measures (Tomasi and Volkow, 2011, Damoiseaux et al., 2008), subject age was included in all group-level analyses.

Target ROIs were converted from standard space into each subject's functional space using previously calculated linear transformations. Mean z-values for the functional connectivity between the mPFC and target ROIs were then extracted. 
A)

EC

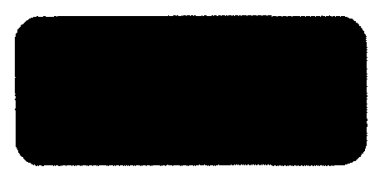

$120 \mathrm{~s}$
EO

$120 \mathrm{~s}$
EC

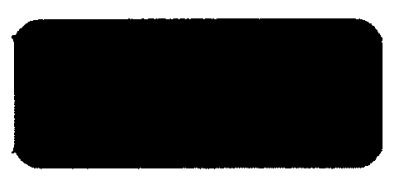

$120 \mathrm{~s}$
EO

$120 \mathrm{~s}$

B)

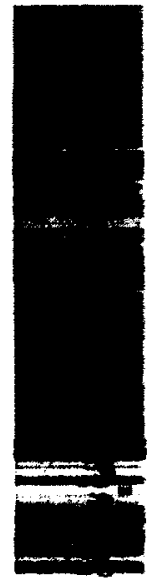

open

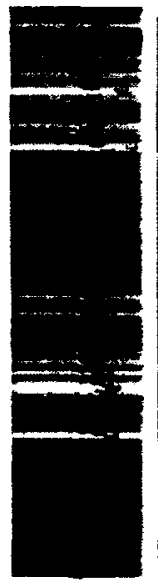

close

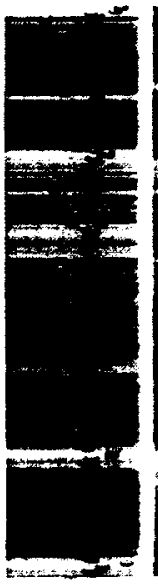

conf

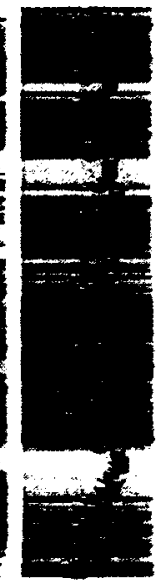

sonif

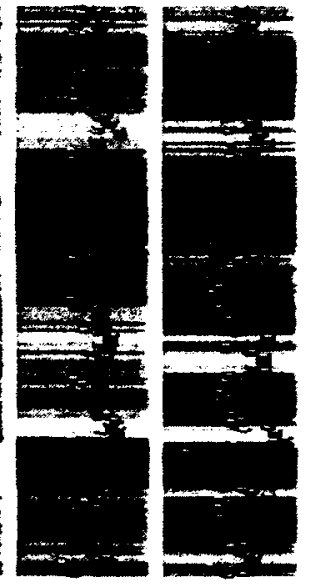

$\cos f$ conf

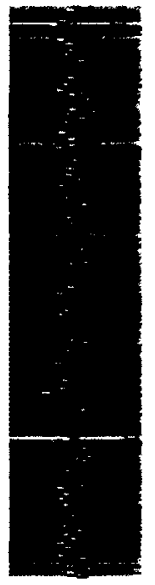

cont

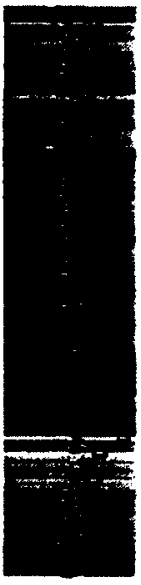

cont

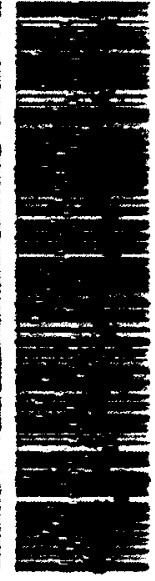

conf conf

Figure 2.4: A) Subjects carried out alternating $120 \mathrm{~s}$ blocks of EO and EC resting-state. B) Sample fMRI analysis design showing mPFC timecourse for EO and EC, along with eight misance regressors (6x head motion (HM). white matter (WM) timecourse. and cerebrospinal fluid (CSF) timecourse). 


\subsubsection{DTI}

Diffusion-weighted images were acquired using a body coil transmit and a 32-channel receive headcoil. Sixty-four slices were aligned parallel to the AC-PC axis and data acquired for 99 diffusion weighting directions with a resolution of $1.9 \times 1.9 \times 1.9 \mathrm{~mm}^{3}$ ( FoV $=243 \mathrm{x}$ $243 \mathrm{~mm}^{2}$, matrix $=128 \times 128$, slice thickness $=1.9 \mathrm{~mm}, \mathrm{~T}_{\mathrm{E}}=89 \mathrm{~ms}, \mathrm{~T}_{\mathrm{R}}=8300 \mathrm{~ms}$, Fourier factor $=6 / 8,99$ acquisitions with $b=1000 \mathrm{~s} / \mathrm{mm}^{2}, 10$ acquisitions with $b=0$ ). In addition, a field map was acquired to correct for field distortions (FoV $=256 \times 256 \mathrm{~mm}^{2}$, matrix $=128 \times 128$, slice thickness $=2 \mathrm{~mm}, \mathrm{~T}_{\mathrm{E} 1}=5.09 \mathrm{~ms}, \mathrm{~T}_{\mathrm{E} 2}=7.55 \mathrm{~ms}, \mathrm{~T}_{\mathrm{R}}=1000$ $\mathrm{ms}$, Fourier factor $=6 / 8$ ).

Raw data were corrected for field distortions, eddy current distortions, and motion artifacts using FSL's FUGUE and eddy_correct tools. DTIFIT was then used to fit a diffusion tensor model at each voxel. Fiber tracking was carried out using Diffusion Toolkit (Wang et al., 2007 ) and an interpolated streamline approach with an angle threshold of $35^{\circ}$. Standard space ROIs were converted to DTI space using linear transformations and the tracks between the mPFC MRS ROI and each of the target ROIs visualised using Trackvis (Wang et al., 2007). The number of tracks, and mean fractional anisotropy (FA) within tracks (weighted by track length), for each ROI pair was obtained from these images.

\subsubsection{Combination of measures}

For each target ROI, the mPFC to ROI FC z-values for each of the EO, EC, and EO>EC conditions were firstly correlated with the level of Glx/NAA in the mPFC, followed by Glu/NAA from the same region. All regions that showed significant correlations with mPFC Glu/NAA were then also tested with left insula Glu/NAA and mPFC Gln/NAA values to show regional and biochemical specificity. Where subjects had tracks linking the $\mathrm{mPFC}$ and a particular target ROI, FC z-values were, in a second step, correlated with both the number of tracks and the mean FA within tracks.

Partial non-parametric correlations were used throughout (Rousselet and Pernet, 2012), taking into account subject age, mPFC ROI grey matter volume, and the proportion of the mPFC ROI in the relevant hemisphere. Significance was set at $p<0.05$ and was tested 
using Monte Carlo simulation (1,000 repetitions). All statistical analyses were carried out in Octave (http://ww. gnu.org/software/octave/).

\subsection{Results}

Correlation details for functional connectivity with mPFC Glu/NAA and DTI measures are given in Table 2.1. Distributions of FC z-values and all scatter plots for mPFC Glu/NAA, and track numbers can be found in the supplementary material (Supplementary figures A.1, A. 2 \& A.3). No regions that showed correlations with mPFC Glx/NAA or Glu/NAA also showed correlations with either of the control mPFC Gln/NAA and left insula Glu/NAA measures (see Supplementary table A.2 for control correlation details).

\subsubsection{NAC}

FC values between the mPFC and the right NAc showed a trend towards a positive correlation with mPFC Glx/NAA ( $\mathrm{r}=0.47, p=0.10)$ during the EO condition that became significant with Glu/NAA $(\mathrm{R}=0.67, p=0.012)$. There were no correlations with the EC condition (although a trend towards a significant positive correlation with Glu/NAA was observed $-\mathrm{R}=0.48, p=0.098$ ), nor with the EO $>\mathrm{EC}$ difference. No correlations with $\mathrm{mPFC}$ Glx/NAA or Glu/NAA were seen in the left NAc during EO, EC, or EO>EC.

Tracks linking the mPFC and the right NAc were observed in twelve subjects and in thirteen with the left NAc, with a mean track number of 33.1 ( \pm 7.9 S.E.M) in the right NAc and 74.4 ( \pm 9.3 S.E.M.) in the left. No correlation was seen between $\mathrm{FC}$ values and any structural measures in either the right or left $N A c$, although a trend to significance was seen between EO FC and mean FA in the left NAc $(\mathrm{R}=-0.52, p=0.068)$.

The degree of FC between the $\mathrm{mPFC}$ and right NAc during the EO condition was thus found to increase with increasing concentrations of $\mathrm{mPFC}$ glutamate. The relationship between FC and Glu/NAA was not found to be mirrored by a correlation between FC and the measures used of structural connectivity between the mPFC and NAc (see Figure 2.5). 


\section{R NAC}
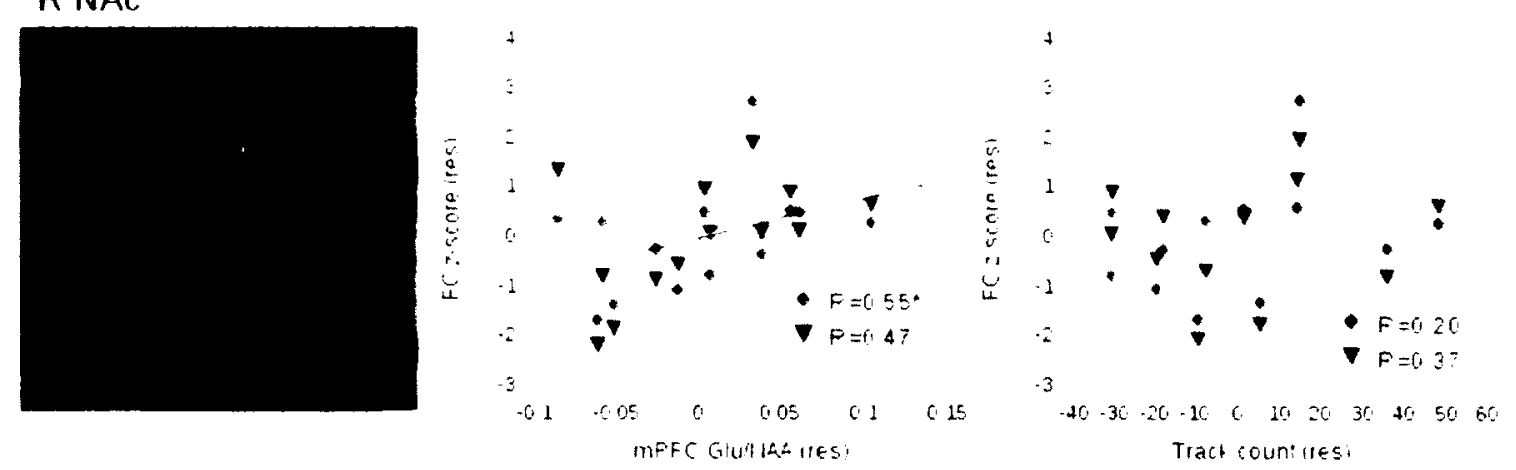

\section{R DMT}
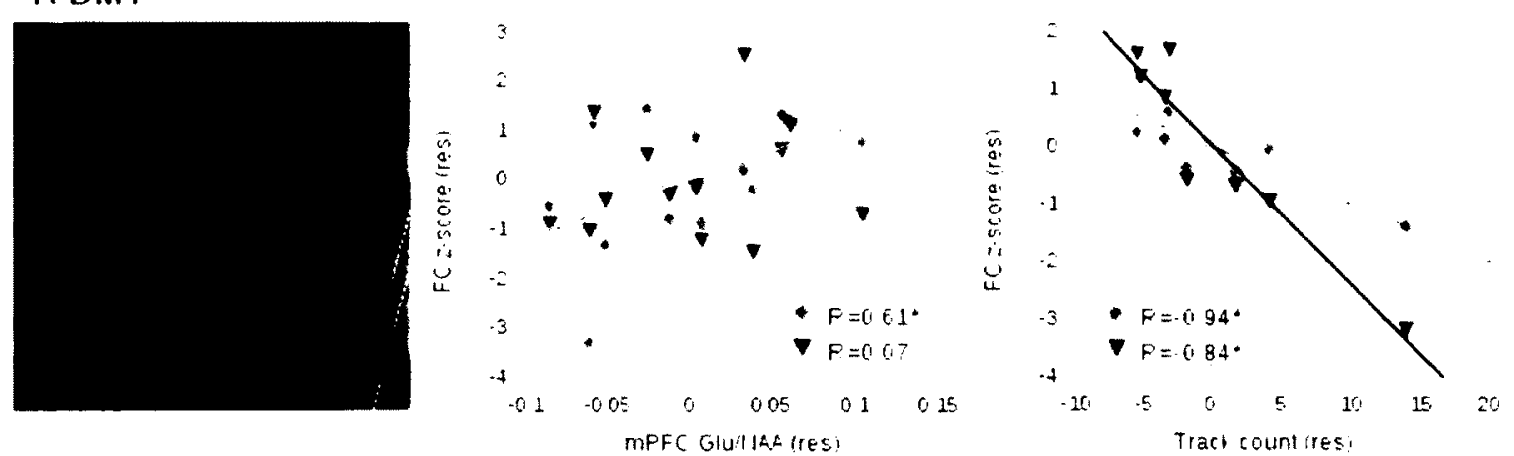

\section{R PAG}
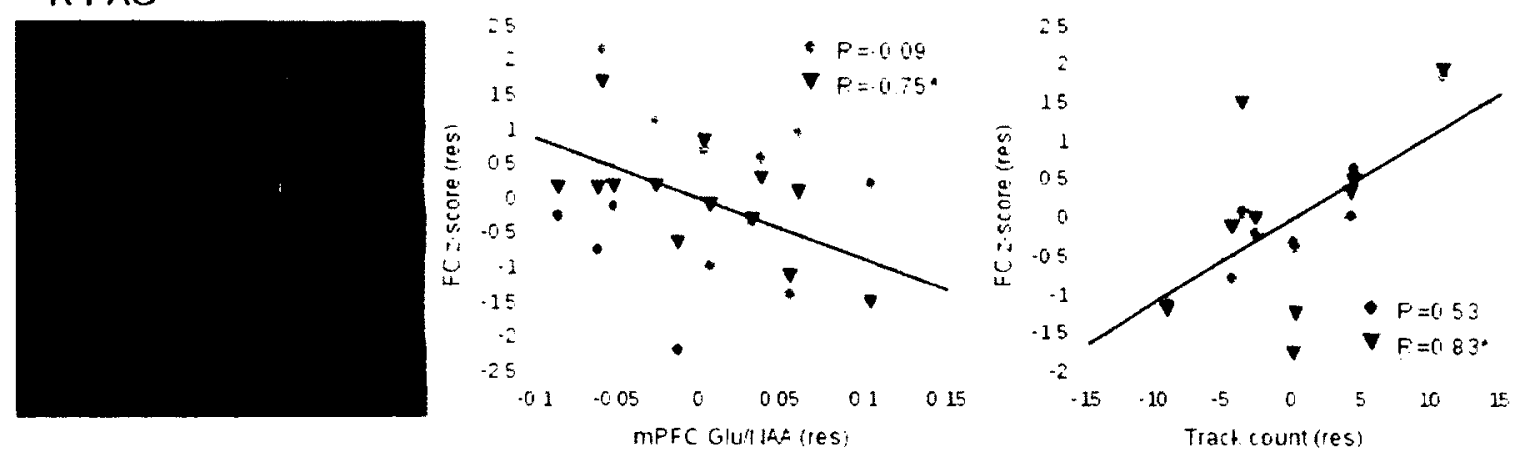

- $E O \quad \nabla E C$

Figure 2.5: Example tracts between the mPFC and each of the target regions are shown along with partial correlation graphs from the right hemisphere. Correlations between FC and Glu are shown, followed by correlations between FC and number of tracts. EO FC correlates with Glu/NAA in both the NAc and DNT whilst EC FC correlates with this in the PAG. Correlations with structural measures can be seen in DNT and PAG but not NAc. Note that values represent residuals after confounding variables have been regressed out of the data in the partial correlation (see Methods). Red diamonds $=$ EO, blue triangles $=$ EC. * indicates $p \therefore 0.05$. 


\subsubsection{DMT}

FC values between the $\mathrm{mPFC}$ and right DMT showed a trend towards a correlation with $\mathrm{mPFC} \mathrm{Glx} / \mathrm{NAA}(\mathrm{R}=0.48, p=0.093)$, and were positively correlated with $\mathrm{mPFC} \mathrm{Glu} / \mathrm{NAA}$ $(\mathrm{R}=0.59, p=0.031)$, during the $\mathrm{EO}$ condition but not the $\mathrm{EC}$ condition. The $\mathrm{EO}>\mathrm{EC}$ difference was also found to be positively correlated with $\mathrm{mPFC}$ Glu/NAA $(\mathrm{R}=0.68, p=$ $0.011)$, with a trend to significance with Glx/NAA $(\mathrm{R}=0.49, p=0.089)$. No correlations were seen in the left DMT, although trends towards significance were seen with Glu/NAA and $\mathrm{EO}(\mathrm{R}=0.41, p=0.16)$ and $\mathrm{EO}>\mathrm{EC}(\mathrm{R}=0.52, p=0.07)$ functional connectivity.

Eight out of thirteen subjects had tracks linking the mPFC and right DMT (mean track number $=22.4 \pm 6.09$ S.E.M), whilst nine out of thirteen subjects had tracks linking the $\mathrm{mPFC}$ and left DMT (mean track number $=57.9 \pm 13.7$ S.E.M). FC values in the right DMT were found to negatively correlate with the number of tracks present, as well as positively with the mean FA within these tracks, during the EO and EC conditions. No relationship between $\mathrm{EO}>\mathrm{EC} \mathrm{FC}$ difference and any structural measure was observed in this region. No correlation was seen between FC in any condition and any of the biochemical or structural measures in the left DMT (see Table 2.1).

As with the NAc, the degree of FC between the mPFC and right DMT during the EO condition was seen to increase with increasing concentrations of mPFC Glu/NAA. In addition, the difference in $\mathrm{FC}$ between the $\mathrm{EO}$ and $\mathrm{EC}$ conditions was also found to increase with increasing Glu concentrations. This relationship was mirrored by a correlation between EO FC and structural measures, with the degree of FC decreasing with a greater number of tracks and increasing as track FA increases (see Figure 2.5).

\subsubsection{PAG}

Right PAG FC correlated negatively with $\mathrm{mPFC}$ Glx/NAA $(\mathrm{R}=-0.62 p=0.024)$ and Glu/NAA in the EC condition ( $\mathrm{R}=-0.75, p=0.0025)$. Positive correlations between the $\mathrm{EO}>\mathrm{EC}$ difference and Glx/NAA $(\mathrm{R}=0.61, p=0.026)$ and Glu/NAA were also seen $(\mathrm{R}$ $=0.7, p=0.0053)$. EC FC in the left PAG was found to correlate negatively with mPFC Glu/NAA $(\mathrm{R}=-0.64, p=0.014)$. 


\begin{tabular}{|c|c|c|c|c|c|c|}
\hline & Glu/NAA & No. Tracks & Mean FA & Glu/NAA & No. Tracks & Mean FA \\
\hline & \multicolumn{3}{|c|}{ Left NAc } & \multicolumn{3}{|c|}{ Right NAc } \\
\hline $\mathrm{EO}$ & $-0.05(0.083)$ & $0.28(0.34)$ & $-0.44(0.11)$ & $0.66(0.012)^{*}$ & $0.2(0.5)$ & $0.07(0.81)$ \\
\hline EC: & $0.28(0.34)$ & $-0.01(0.97)$ & $-0.42(0.13)$ & $0.48(0.098) \dagger$ & $0.37(0.21)$ & $0.08(0.79)$ \\
\hline \multirow[t]{2}{*}{$\mathrm{EO}>\mathrm{EC}$} & $-0.40(0.17)$ & $0.25(0.38)$ & $-0.09(0.77)$ & $0.41(0.16)$ & $-0.32(0.29)$ & $-0.02(0.94)$ \\
\hline & \multicolumn{3}{|c|}{ Left DMT } & \multicolumn{3}{|c|}{ Right DMT } \\
\hline EO & $0.41(0.15)$ & $-0.62(0.08) \dagger$ & $0.38(0.31)$ & $0.59(0.031)^{*}$ & $-0.94(<0.001)^{*}$ & $0.97(<0.001)^{*}$ \\
\hline EC & $0.22(0.45)$ & $-0.61(0.08) \dagger$ & $0.97(<0.001)^{*}$ & $0.07(0.82)$ & $-0.84(<0.001)^{*}$ & $0.96(<0.001)^{*}$ \\
\hline \multirow[t]{2}{*}{$\mathrm{EO}>\mathrm{EC}$} & $0.52(0.07) \dagger$ & $0.08(0.83)$ & $-0.45(0.23)$ & $0.67(0.011)^{*}$ & $-0.67(0.06) \dagger$ & $0.55(0.15)$ \\
\hline & \multicolumn{3}{|c|}{ Left PAG } & \multicolumn{3}{|c|}{ Right PAG } \\
\hline EO & $-0.28(0.39)$ & $0.23(0.5)$ & $-0.4(0.23)$ & $-0.09(0.74)$ & $0.5(0.14)$ & $0.19(0.59)$ \\
\hline EC & $-0.64(0.021)^{*}$ & $0.05(0.89)$ & $-0.23(0.49)$ & $-0.75(0.006)^{*}$ & $0.83(0.003)^{*}$ & $-0.08(0.83)$ \\
\hline $\mathrm{EO}>\mathrm{EC}$ & $0.24(0.50)$ & $0.37(0.27)$ & $-0.48(0.13)$ & $0.67(0.011)^{*}$ & $-0.47(0.17)$ & 0.29 \\
\hline
\end{tabular}

Table 2.1: Correlations between $\mathrm{mPFC}$ to target FC and mPFC Glu/NAA, number of connecting tracts, and mean tract $\mathrm{FA}$, in the left and right NAc, DMT, and PAG. R-values are given with $p$-values in parenthesis. * indicates statistical significance, $\dagger$ denotes a trend to significance. 
Nine out of thirteen subjects had tracks linking the mPFC and right PAG (mean track number $=6.2 \pm 1.98 \mathrm{~S} . \mathrm{E} . \mathrm{M}$ ), with the same number having tracks linking the mPFC and left PAG (mean track number $=14.36 \pm 6.21$ S.E.M). Right PAG EC FC was positively correlated with track number $(\mathrm{R}=0.83, p=0.0028)$, although no relationship was seen with FA values. No correlations between left PAG FC and structural measures were observed (Table 2.1).

In summary, the degree of FC between the mPFC and right PAG during the $\mathrm{EC}$ condition decreased with greater mPFC Glu/NAA concentrations. With an increase in the number of tracks between the MPFC and PAG there was an increase in the degree of FC (see Figure 2.5). The same indirect relationship between Glu/NAA and track number in relation to FC was thus seen in the PAG as in the DMT (i.e., lower track counts with higher Glu/NAA).

\subsection{Discussion}

We here show that the concentration of glutamate in the mPFC predicted the degree of FC between this region and various subcortical regions (the right NAc, DMT, and PAG). FC was also found to correlate with measures of structural connectivity between the MPFC and the DMT and PAG, but not NAc, hence providing evidence that the FC with these regions may be a result of direct anatomical communication (see Figure 2.6 for an overview of results). The inclusion of the left insula as a control region further supports the regional specificity of the results. These findings show, for the first time in humans, that the strength of cortical-subcortical connectivity is associated, in part, with cortical glutamate concentrations. This complements previous studies using non-human animals (see below) and may have important implications for our understanding of brain networks and psychiatric disorders.

\subsection{1 mPFC glutamate and subcortical functional connectivity}

Both structural (Roberts et al., 2007, Groenewegen et al., 1999, Johansen-Berg et al., 2005, Hadjipavlou et al., 2006) and functional connectivity (Zhang et al., 2008, Mainero et al., 2011, Margulies et al., 2007) between the mPFC and each of the subcortical target 


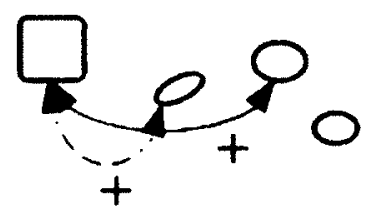

EC

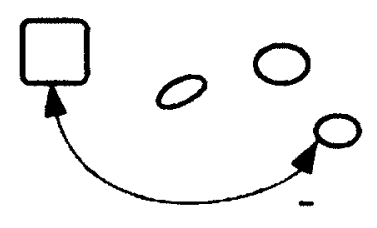

\section{$E O>E C$}
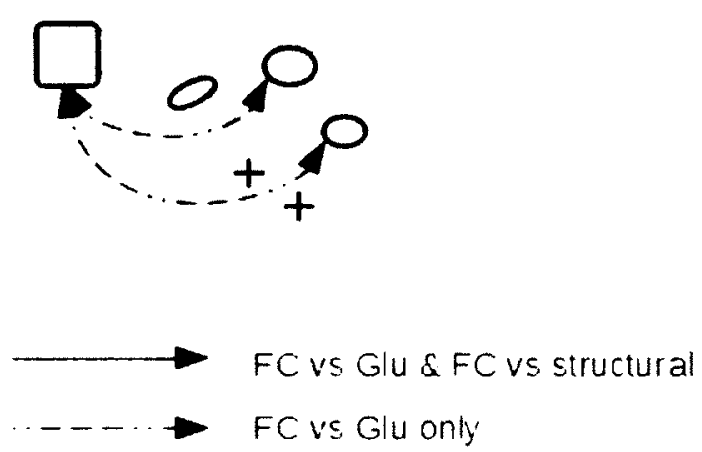

Figure 2.6: Overview of comnectivity in EO. EC and EO EC conditions. Shown are the mPFC, NAc, DMT, and PAG. Black lines denote that a relationship between FC and both Glu NAA and structural measures are seen in the relevant condition. Dashed lines denote that a relationship between FC and Glu NAA only is seen. symbol beside arrow denotes a positive correlation with Glu/NA. - symbol beside arrow denotes a negative correlation with Glu NAA. 
regions has been demonstrated previously through a combination of human and non-human imaging and post-mortem studies. The mPFC itself is rich in glutamatergic cells and innervation (Bozkurt et al., 2005, Palomero-Gallagher et al., 2009) and has been identified as an important site for the mediation of the behavioural effects of glutamatergic agents (Feenstra et al., 2002). In both humans and animals, mPFC task-related activity is altered when glutamatergic agents (such as ketamine) are administered (Musso et al., 2011, Fu et al., 2005, Salvadore et al., 2010, Jodo et al., 2010, Katayama et al., 2007, Jackson et al., 2004), as is functional connectivity between the mPFC and other brain regions (Dawson et al., 2011, Salvadore et al., 2010). The current findings thus fit well into the patterns of connectivity and biochemistry known for the mPFC. In addition, these previous findings lend weight to the conclusion that the relationship between Glu/NAA and FC seen here is at least partly related to direct connectivity in the PAG and DMT (the regions that show a correlation between FC and structural measures).

Glutamate has also been shown to play in important role in the function of each of the target regions (often in conjunction with other transmitters). In the NAc, glutamatergic inputs come from multiple cortical and subcortical sites, including the mPFC (Groenewegen et al., 1999, Margolis et al., 2006, Morgane et al., 2005). The role of glutamate within the NAc in relation to behaviour has been well established, as has the effect of glutamatergic agents on its biochemical functioning, such as on dopamine release, and on it's effect on behaviour, such as on movement arousal (Del Arco and Mora, 2009, Schmidt and Pierce, 2010, Feenstra et al., 2002). Important in this modulation of NAc functioning appears to be the interaction between the mPFC and NAc (Gruber et al., 2009), where glutamatergic communication plays a key role (Del Arco and Mora, 2005, 2009, Feenstra et al., 2002). Glutamate also plays a role in communication between the DMT and mPFC, as is demonstrated in a number of animal studies, and by glutamatergic substances producing correlated changes in the activity of each region (Jodo et al., 2005, Kargieman et al., 2007, Dawson et al., 2011, Lå ngsjö et al., 2004). Within the DMT itself, glutamate is a key neurotransmitter (Kargieman et al., 2007). Finally, the $\mathrm{mPFC}$ has been identified as an important source of glutamateric projections to the PAG (Coutinho and Menescal-de Oliveira, 2010, An et al., 1998, Butler et al., 2011). These connections appear to be important for the cortical modulation of pain and fear responses mediated by the PAG (Mainero et al., 2011, Coutinho and Menescal-de Oliveira, 2010).

Resting-state functional connectivity itself is a measure of the temporal synchronisation 
between the activity in different brain regions. In humans, FC studies have focused on a range of frequencies, from spontaneous BOLD fluctuations in low frequency ranges (Cole et al., 2010, Smith et al., 2011) to higher frequencies in, for example, EEG or MEG (Martini et al., 2012, Chen et al., 2012). The role of glutamate in functional connectivity in humans has not been widely studied to date, although some preliminary studies have been carried out (Duncan et al., 2011, Horn et al., 2010). In animals, both ionotropic NMDA and metabotropic glutamate receptors have been shown to be involved in brain-wide synchronisation of activity at a range of fluctuation frequencies (Whittington et al., 1995, Papatheodoropoulos, 2007, Fellin et al., 2004), although it is worth noting that this action is likely to be in conjunction with other transmitters, such as GABA (Louvel et al., 2001). In addition, glutamatergic agents like phencyclidine and ketamine have been shown to reduce cortical synchrony in humans (Kargieman et al., 2007, Salvadore et al., 2009). The current study thus fits into a background of evidence that glutamate is involved in the temporal synchronisation between regions across the brain and builds on it by demonstrating such a role in relation to specific regions.

The correlations present between mPFC Glu and FC during the EC condition no longer held during EO. Such changes in brain activity properties between the EO and EC resting-state have been described previously in humans (Donahue et al., 2011, ?, Marx et al., 2004, ?, Wiesmann et al., 2006, Yang et al., 2007). Interestingly, not all of the changes observed are due to a simple increase in visual stimulation in EO, as changes are also seen in complete darkness and in congenitally blind subjects (Hüfner et al., 2008, 2009, Wiesmann et al., 2006). Compared to the EC condition, EO induces a reduction in global alpha activity, along with an increase in skin-conductance (Barry et al., 2007, 2011), increased activity in the DMT (Marx et al., 2003), and a brain-wide (although mPFC focused) increase in FC (Chen et al., 2012). Such changes, particularly those in alpha activity, are likely to be linked to an increase in arousal or attention, broadly construed, as the eyes are opened, with a concurrent change in functional organisation across the brain (Barry et al., 2011, Chen et al., 2012, Klimesch, 1999). Given this, one could hypothesise that the changes in the relationship between mPFC Glu and FC between EO and EC seen here are due in part to a switching in arousal or attention, and as such in the organisation of neural activity, as the eyes are opened or closed. This would be in line with the established role for the $\mathrm{mPFC}$ in the modulation of arousal in humans (Nagai et al., 2004, Zhang et al., 2012) and with $\mathrm{mPFC}$ glutamate-related modulation of arousal in rats (David, 2009, Feenstra et al., 
2002). Such a process would also provide a possible explanation for the notable rightlateralisation of the current $\mathrm{NAc}$ and thalamus results, as previous studies have shown the importance a strongly right-lateralised network in arousal and attention, that includes the mPFC, thalamus and striatum, in arousal and attention (Coull, 1998, Marx et al., 2003). Research with direct measures of arousal, such as skin conductance, are however required to explore this possibility further.

\subsubsection{Implications for MDD and schizophrenia}

Both major depressive disorder (MDD) and schizophrenia have been related to changes in glutamatergic function (Corti et al., 2011, Hashimoto, 2009, Volk et al., 2010) and to altered activity in the mPFC (Alcaro et al., 2010, Kühn and Gallinat, 2011). In the case of MDD, hyperactivity in the mPFC during the resting state has been described in humans and animals, with this hyperactivity being concurrent with hyperactivity in a set of subcortical regions that includes the NAc, DMT, and PAG (Alcaro et al., 2010, Northoff et al., 2011). In

addition to these activity changes in MDD, changes in $\mathrm{MPFC}$ Glu and glutamate receptors in MDD have been observed in humans and animals (Gibbons et al., 2012, Hasler et al., 2007, Walter et al., 2009, Zhao et al., 2012). In this context, our findings that mPFC glutamate is related to $\mathrm{FC}$ between the $\mathrm{MPFC}$ and subcortical regions is suggestive of a role for this transmitter in altered cortical-subcortical regulation in MDD. The anti-depressant effects of glutamatergic substances such as ketamine could then be hypothesised to involve a re-balancing action on such alterations (Hashimoto, 2009).

As with MDD, glutamatergic alterations and the action of glutamatergic substances have been linked to schizophrenia (Stone et al., 2012). In the mPFC specifically, changes in glutamatergic receptors and uptake proteins have been reported (Corti et al., 2011, Volk et al., 2010). However, conversely to MDD, mPFC resting activity appears to be decreased in schizophrenia (Kühn and Gallinat, 2011, Lesh et al., 2011). These mPFC changes have then been suggested to impact upon cortico-striatal-thalamic loops, producing imbalances in brain networks that lead to the symptoms of the condition (Cronenwett and Csernansky, 2010, Marek, 2010). The involvement of the thalamus implied by such modulation of corticostriatal-thalamic loops links with the current findings and is supported by a wide range of evidence, including structural and biochemical (Clinton and Meador-Woodruff, 2004, Cronenwett and Csernansky, 2010, Spoletini et al., 2011), as well as changes in resting-state FC 
(Skudlarski et al., 2010). In addition to the thalamus, striatum involvement in schizophrenia, and specifically the $\mathrm{NAc}$, has been indicated by altered cortico-striatal dopamine regulation, with this in turn linked to schizophrenic-type symptoms (Aalto et al., 2005, George et al., 2011). The connection made here between glutamate and cortico-striatal communication could thus be taken in the context of mPFC-NAc glutamate-dopamine interaction (Del Arco and Mora, 2005, 2009, Feenstra et al., 2002) to provide a tentative link between the roles of these transmitters in schizophrenia. As with MDD, then, the current findings fit into a pathology scenario that involves dysregulation of cortical-subcortical networks and dysfunctional glutamatergic communication (Menon, 2011, Northoff et al., 2011), and as such point towards potentially interesting avenues of research into glutamate-related cortico-subcortical network function in humans.

\subsubsection{Limitations \& conclusion}

A number of limitations of the current study must be taken into account. Firstly it is worth noting that the $\mathrm{mPFC}$ region studied was quite large (to allow the use of the MEGA-PRESS method), being mainly centred upon the pgACC, extending forward to include a portion of BA 10 , and also covering the white matter lateral to the ACC. The anatomical specificity of the results is therefore somewhat limited. Though the current interpretation may benefit from future studies using smaller MRS regions (when feasible) or higher fields (like $7 \mathrm{~T}$ ), the present findings are nonetheless largely supported by findings in both the human and animal literature. Similarly, with the current resolution we cannot differentiate between, for example, the shell and the core of the NAc, or different DMT nuclei. The lack of resolution in the NAc may explain the dissociation between the glutamate and DTI results through the differences in innervation between these subdivisions(Asher and Lodge, 2011, Groenewegen et al., 1999), although this is speculative. Secondly, FC is not a directional or causative measure, nor does it give any information about the relative intensity of activity in different regions. Instead it gives information only about whether the BOLD signal is more or less synchronised with that in the seed region. These features mean that one must be hesitant in making too strong a claim about the meaning of any $\mathrm{FC}$ result. For example, although in the current study a relationship between mPFC Glu/NAA and NAc FC is seen, the NAc also receives input from the DMT (Jones, 2009). The relationship seen in the NAc may therefore be due to an indirect synchronisation in the BOLD signal mediated 
by a third region, such as the DMT (or indeed by a region with input to both seed and target not analysed here, such as the ventral tegmental area (Björklund and Dunnett, 2007, Margolis et al., 2006)). Finally, the final study group was somewhat small as the data from a number of participants had to be rejected due to problems with either head-movement or with spectral quality.

To conclude, a role for mPFC glutamate in functional connectivity between the cortex and subcortical regions (specifically the NAc, DMT, and PAG) in humans was shown. In the DMT and PAG this was mirrored by a correlation between FC and structural connectivity measures, suggesting that the glutamatergic relationship is due to direct anatomical connectivity in at least these regions. These results extend existing results from non-human animal studies and may have relevance to a range of psychiatric conditions, including MDD and schizophrenia.

\subsection{Acknowledgements}

Many thanks to A. Perna and K. Dedovic for their help with subject recruitment, Edward J. Auerbach, Ph.D. (Centre for Magnetic Resonance Research, University of Minnesota) for implementing the MEGA-PRESS sequence on Siemens, the staff at the UNF and MNI for their skillful assistance, and Romain Valabregue, Ph.D. (Centre de Neurolmagerie de Recherche, Paris, France) for developing processing tools. The work was supported by grants to G.N. from the Canadian Institutes of Health Research and the Michael Smith Foundation. 


\section{Chapter 3}

\section{Involvement of glutamate in rest-stimulus interaction between perigenual and supragenual anterior cingulate cortex}

\subsection{Abstract}

The brain shows a high degree of activity at rest. The significance of this activity has come increasingly into focus. At present, however, the interaction between this activity and stimulus-induced activity is not well defined. The interaction between a task-negative (perigenual anterior cingulate cortex, pgACC) and task-positive (supragenual anterior cingulate cortex, sgACC) region during a simple task was thus investigated using a combination of AMRI and MRS. Negative BOLD responses in the pgACC were found to show a unidirectional functional connectivity with task-induced positive BOLD responses in the sgACC. This connectivity was shown to be related specifically with glutamate levels in the pgACC. These results demonstrate an interaction between deactivation from resting-state and resting-state glutamate levels in a task-negative region $(\mathrm{pgACC})$, and task-induced activity in a task- positive region ( $\mathrm{sg} A \mathrm{CC}$ ). This provides insight into the neuronal and biochemical mechanisms by means of which the resting state activity of the brain potentially impacts upon subsequent stimulus-induced activity.

\subsection{Introduction}

The significance of high resting-state activity in the brain has been the focus of increasing interest (Carhart-Harris and Friston, 2010, Northoff et al., 2010a, Raichle, 2009). Prominent in these investigations have been the set of regions, known as the default-mode network 
(DMN), that show a consistent pattern of deactivation from this high resting-state activity in response to the presentation of tasks or stimuli, as well as a high degree of functional connectivity during rest (Beckmann et al., 2005, Broyd et al., 2009, Buckner et al., 2008, Raichle et al., 2001). This pattern of deactivation has led to these regions being referred to as task-negative regions, distinguishing them from task-positive regions, in which positive BOLD responses are seen in response to specific task types.

Studies have demonstrated that resting-state activity has an impact upon stimulus-induced responses within both task-negative (Northoff et al., 2007) and task-positive regions (Arieli et al., 1996, Boly et al., 2007, Fox and Raichle, 2007, Maandag et al., 2007, Muthukumaraswamy et al., 2009) in animals and humans. In addition, the existence of resting-state functional interconnection between task-positive and task-negative regions is increasingly well supported (Hampson et al., 2010, Taylor et al., 2009). These functional connectivity findings build on the described anti-correlations between activity in task-positive and tasknegative regions (Cole et al., 2010, Fox et al., 2005). What remains unclear, however, is how resting-state dependant activity in task-negative regions impacts and interacts with stimulus-induced activity in task-positive regions. Similarly, the biochemical mechanisms underlying such interactions remain to be investigated.

The general aim of this study consisted in the investigation of the neuronal and biochemical mechanisms underlying the interaction between the deactivation from resting-state in a task-negative region and stimulus-induced activity in a task-positive region. The tasknegative region of interest, the perigenual anterior cingulate cortex (pgACC), forms part of the DMN and displays a characteristic task-induced deactivation (Buckner et al., 2008, Grimm et al., 2009, Northoff et al., 2007). In contrast, the task-positive region of interest, the supragenual anterior cingulate cortex ( $\mathrm{sgACC}$ ), displays positive BOLD responses in response to a number of task types (e.g. Aron, 2007, Carter and van Veen, 2007, Preston and de Waal, 2002). These regions were selected due to their identification as typical tasknegative and task positive regions, respectively, that display close functional and anatomical connectivity (Ongür and Price, 2000). A simple empathy task was selected as a probe, with this task type having been consistently observed to elicit a negative BOLD response in the pgACC (Grimm et al., 2009) and a positive BOLD response in the sgACC (Prehn-Kristensen et al., 2009, Singer and Lamm, 2009).

The contrast between task-induced positive and negative responses in the regions of interest 
reflects the well documented variation in anatomy and connectivity throughout the anterior cingulate cortex (ACC) (Beckmann et al., 2009, Gittins and Harrison, 2004, Margulies et al., 2007, Palomero-Gallagher et al., 2008, Vogt et al., 1995). Both regions studied here do, however, display a similarly rich glutamatergic innervation (Bozkurt et al., 2005, Palomero-Gallagher et al., 2009). This neurotransmitter system, as the main excitatory neurotransmitter in the cortex, is thus likely to play a role in any interaction between the two regions. As such, glutamatergic function in both pgACC and sgACC was indirectly measured (as a combined value for glutamate and its precursor, glutamine) using magnetic resonance spectroscopy (MRS) to test for the biochemical mechanisms of possible reststimulus interaction between both regions.

Based on the above described evidence, it was hypothesised that task-induced negative $B O L D$ responses in the $\mathrm{pg} A C \mathrm{C}$ would interact with task-induced positive BOLD responses in the sgACC, and that this interaction would be mediated by glutamatergic communication. To ensure that the results gained were specific to both the regions and task-type studied, rather than being examples of global effects, a control MRS region (the left anterior insula) and control tasks (a simple reward task and an emotion evaluation task) were employed.

\subsection{Methods}

\subsubsection{Participants}

Thirteen healthy subjects with no psychiatric, neurological or medical illnesses were studied (9 female; average age 31.6 years, range 22 to 59 years; 11 right handed). After a detailed explanation of the study design and potential risks, all subjects gave their written informed consent. fMRI and MRS sessions were carried out on subsequent days in a randomised order. Participants had taken no medication or caffeine prior to either scanning session. Each of the 13 subjects completed both fMRI and MRS. The study was approved by the institutional review board of the University of Magdeburg, Germany. 


\subsubsection{Experimental paradigm}

The fMRI scanning session was divided into six scanning runs. In three of these runs $(2,4$ and 6) the empathy task was displayed, alternating with three runs (1,3 and 5) in which a reward task was shown. Prior to entering the scanner each subject completed a number of trial presentations of the tasks in order to familiarise them fully with them. In the scanner, images were displayed using the Presentation software package (Neurobehavioural Systems, Albany, CA), and were projected onto a screen visible through a mirror mounted on the headcoil via an LCD projector.

\section{Empathy task}

In the first component of each empathy trial, subjects were presented for $5 \mathrm{~s}$ with pictures from the Matsumoto and Ekman's Japanese and Caucasian Facial Expressions of Emotion (Matsumoto and Ekman, 1988), with a balanced number of Caucasian and Japanese faces being displayed. Subjects were instructed to view the pictures and empathise with the person represented. Faces showed either a happy, angry, disgusted, or neutral expression, ordered randomly. All instances of these four emotion types, and both Caucasian and Japanese faces, were grouped in the analysis as a single emotional viewing condition (empathy). Also displayed were smoothed versions of these pictures to provide a control condition with no emotional content (view smoothed) that was matched for colour composition and intensity, as well as for overall visual structural properties. Pictures without any emotional content were used as the comparison condition as, following a recent hypothesis, the mere perception of emotional faces, including neutral, should be sufficient for inducing an empathic response (Preston and de Waal, 2002).

Following picture presentation, an evaluation condition (empathy evaluation) was displayed for $5 \mathrm{~s}$. This consisted of a German text ("Ich konnte mich in die gezeigte Person hineinversetzen") asking to what degree the subject could empathise with the person in the prior picture, along with a sliding visual analogue scale. Subjects moved the indicator to the left (not at all) or to the right (completely) to indicate how well or otherwise they could empathise using a two-button feedback device. 
An inter-trial interval of 2-3 s occurred after each picture display and evaluation sequence was presented. These consisted of a dark cross on a light background, with participants being instructed to fixate on the cross during the ITI. In addition, five fixation-cross periods of 6-8 s were located randomly in each run. These conditions were included in the design matrix as a separate condition (fixation), and used in the subsequent analysis as an operationalisation of the resting state condition.

Each of the three runs contained 32 instances of emotional faces, 8 instances of smoothed faces, and 5 instances of the long fixation cross condition, giving a total number of instances of each of 96,24 and 15, respectively (one subject aborted the final empathy task run before its completion - the data for the two empathy task runs that this subject did complete were included in the analysis).

A comparison between the results obtained using the above described paradigm and a meta-analysis of empathy studies was carried out to ensure the paradigm's validity. The meta-analysis was carried out using the MKDA software package (Kober et al., 2008), and included coordinates from a total of 42 studies of empathy in healthy adults. A close match between the regions identified in the current study from the contrast [view empathy $>$ view control] and those identified in the meta-analysis was observed (Figure 3.1), providing some validation for the paradigm. (See Fan et al. (2011) for a full report of this meta-analysis.)

\section{Reward task}

The reward task was a modified version of the well-established monetary incentive delay task (Knutson et al., 2000), requiring that the subject press a button with the index finger of their right hand within a certain time of a target image (a black square in the centre of the screen) being displayed. The reward task was used only as a control task in this analysis, allowing the specificity of the particular findings discussed here for empathy to be tested. 


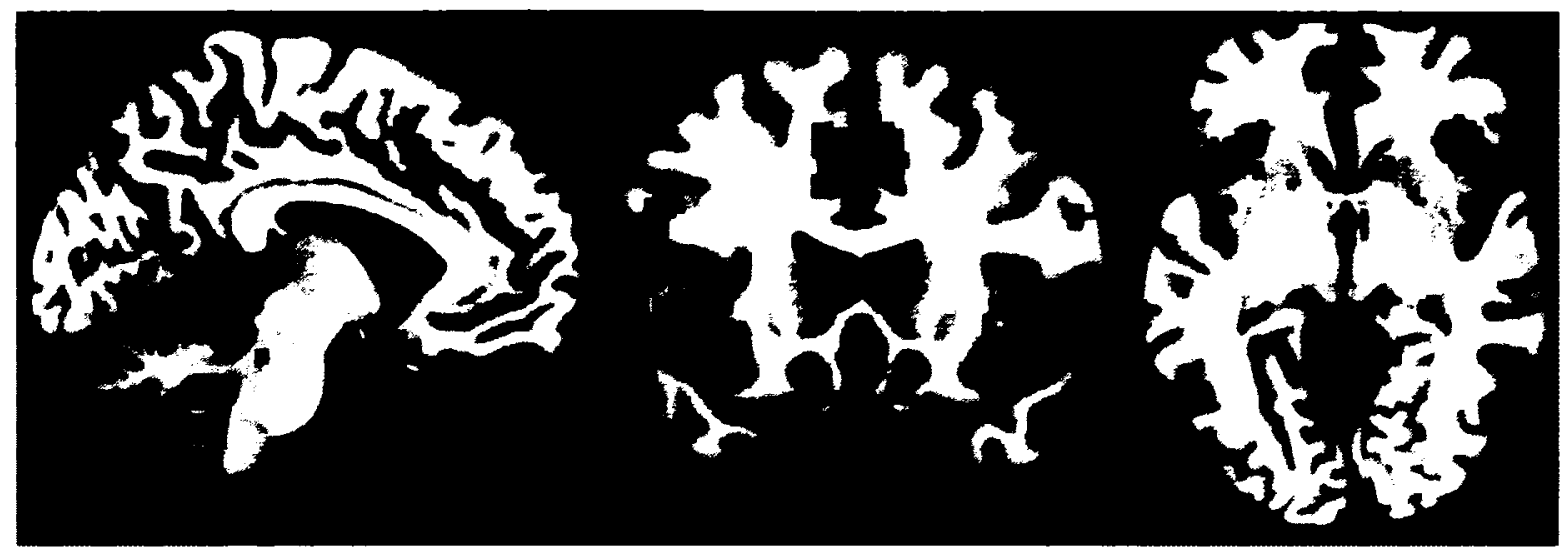

Figure 3.1: Overlap between fMRI experiment and meta-analysis results. fMIRI results shown in red, meta-analysis results shown in blue, overlap shown in purple. An extensive overlap between the fAIRI and meta-analysis in both the sgACC, including the sgACC ROI used in the analysis of the fMRI data. and anterior insula. Due to the small number of studies reporting deactivations, no metaanalytic: data is arailable for the pgACC.

\subsection{3 fMRI data acquisition and analysis}

The fMRI component of the study was carried out on a 1.5 Tesla MR scanner (General Electric Sigma Horizon) using the standard circular polarised headcoil. Using a midsagittal scout image. a stack of 23 slices was aligned parallel to the bicomissural plane. During each functional run 320 whole brain volumes were acquired (gradient echo EPI, $T_{R}-2 s: T_{E} \quad 35$ ms: flip angle -- $80^{\circ}$; FoV - $200 \times 200 \mathrm{~mm}$ : slice thickness - $5 \mathrm{~mm}$, interslice gap - $1 \mathrm{~mm}$, spatial resolution $=3.125 \times 3.125 \times 5 \mathrm{~mm}^{3}$ ). Image processing and statistical analyses were carried out according to the general linear model approach using the SPM8 sof ware package (Wellcome Department of Imaging Neuroscience, London, CK) ruming on MATLAB (The Mathworks, LSA). The first five volumes were discarded due to saturation effects. All functional images were slice-time corrected with reference to the first slice acquired. corrected for motion artifacts by realigmment to the first volume (with the movement parameters obtained in this stage being included in each first-level analysis as separate regressors), and spatially normalised to a standard $T_{1}$-weighted SPMI template (Ashburner and Friston, 1999). The images were resampled to $2 \times 2 \times 2 \mathrm{~mm}^{3}$ and smoothed with an isotropic $8 \mathrm{~mm}$ full-width half maximum Gaussian kernel. The time-series fMRI data were filtered using a 
high pass filter and cut-off of $128 \mathrm{~s}$. A statistical model for each subject was computed by applying a canonical haemodynamic response function. Regionally specific condition effects were tested by employing linear contrasts for each subject and each condition of interest. The resulting contrast images were submitted to a second-level random-effects analysis by applying a one-sample t-test to the images acquired for all subjects in each condition. Resulting clusters of activation were only considered if they had a p-value of less the 0.05 following correction for multiple comparison errors (FWE-correction).

\section{Connectivity analysis}

Functional connectivity between the pgACC and sgACC during the empathy task was tested using psychophysiological interaction (PPI) analysis (Friston et al., 1997). Such analysis allows the identification of those regions that display an increased coupling with a specific seed region in response to a particular psychological factor. In this case, the NBR signal change from resting state within the pgACC in response to the viewing of emotional pictures was taken as the physiological variable of interest. By performing multiple correlation and regression analyses, a PPI analysis then searches for those signal changes in other regions that show a greater correlation with those in the seed region during the condition of interest, as compared to the contrasting psychological variable. This allows, in the case of this study, for the connectivity of a region which displays a negative BOLD response (NBR) during a task with those areas that display a positive BOLD response (PBR) to be determined. As the level of resting state activation has been shown to influence the NBR produced by stimuli (Northoff et al., 2010b), this approach also allows an indirect characterisation of the interaction between the resting state in the pgACC and stimulus-induced signal changes in other regions.

Seed volumes of interest (VOI) corresponding to the pgACC MRS voxel were used to extract the eigenvariate for each subject - the physiological variable - within this region from the relevant source contrasts ([fixation > empathy]; [fixation > evaluate empathy]; [fixation > reward]) using the SPM8 'eigenvariate' function. Having defined and extracted data from these regions, three separate PPI analyses were carried out for each subject. The first of these investigated the change in functional coupling with the pgACC seed region (using the

signal changes from [fixation > view empathy]) between the viewing of emotional pictures and the viewing of the control smoothed pictures. Statistical maps from the individual PPI 
analyses were combined into a group analysis through a one sample t-test in SPM. This process thus identified those regions that display functional connectivity with the pgACC during empathy. Finally, the same procedure was followed using the sgACC MRS voxel as the seed region.

In order to determine the specificity of any connectivity, two further PPI analyses were then carried out. The specificity of the connectivity between the pgACC and sgACC for empathy, as opposed to the evaluation of empathy, was tested by carrying out a PPI using the pgACC signal change from the [fixation > evaluate empathy] contrast as physiological variable and the evaluation of emotional pictures and evaluation of smoothed pictures as psychological variables. Finally, to determine the specificity of the connectivity for empathy, as opposed to other psychological functions, a PPI using the signal changes in the pgACC from the contrast [fixation > reward] as the physiological variable and the anticipation of reward and the anticipation of no outcome as psychological variables.

\subsubsection{MRS data acquisition and analysis}

Single voxel ${ }^{1} \mathrm{H}$ MR spectra were acquired during the resting state using a 3 Tesla whole body MRI system (Siemens Trio) using an eight channel head coil (PRESS, $\mathrm{T}_{\mathrm{R}}=2 \mathrm{~s} ; \mathrm{T}_{\mathrm{E}}=$ $80 \mathrm{~ms}$ ). Voxels were prescribed on a high resolution $\mathrm{T}_{1}$-weighted $3 \mathrm{D}$ data set (MPRAGE, $\mathrm{T}_{\mathrm{R}}=2 \mathrm{~s} ; \mathrm{T}_{\mathrm{I}}=1.1 \mathrm{~s} ; \mathrm{T}_{\mathrm{E}}=4.8 \mathrm{~ms} ;$ Alip angle $=7^{\circ} ; \mathrm{FoV}=256 \times 256 \times 192 ;$ spatial resolution $=1 \times 1 \times 1 \mathrm{~mm}^{3}$ ). One voxel of $20 \times 10 \times 20 \mathrm{~mm}^{3}$ was placed in the bilateral pgACC, whilst a second was placed in the bilateral $\operatorname{sgACC}\left(20 \times 10 \times 20 \mathrm{~mm}^{3}\right)$. A third voxel was placed in the left anterior insula $\left(15 \times 10 \times 20 \mathrm{~mm}^{3}\right)$ in order to provide a control region to establish if any potential relationship between ACC activity and global Glx/Cr levels existed. Voxels were located in relation to relevant anatomical landmarks, as identified by the researcher on a high resolution $T_{1}$-weighted anatomical image (Figure 3.2)

Spectra were eddy current corrected and analysed using LC Model version 6.1.0. Included in the model build were creatine, $\mathrm{N}$-acetyle aspartate, choline, glutamate plus glutamine, and (myo-)inositol. Spectra with full-width-half-maximum line widths larger than $8 \mathrm{~Hz}$ and quantification results with a Cramér-Rao lower bound higher than $20 \%$ were excluded from further analysis. The measurements for one subject in each of the pgACC, sgACC and left insula were discarded for these reasons $(n=12)$. Metabolite concentrations are given 

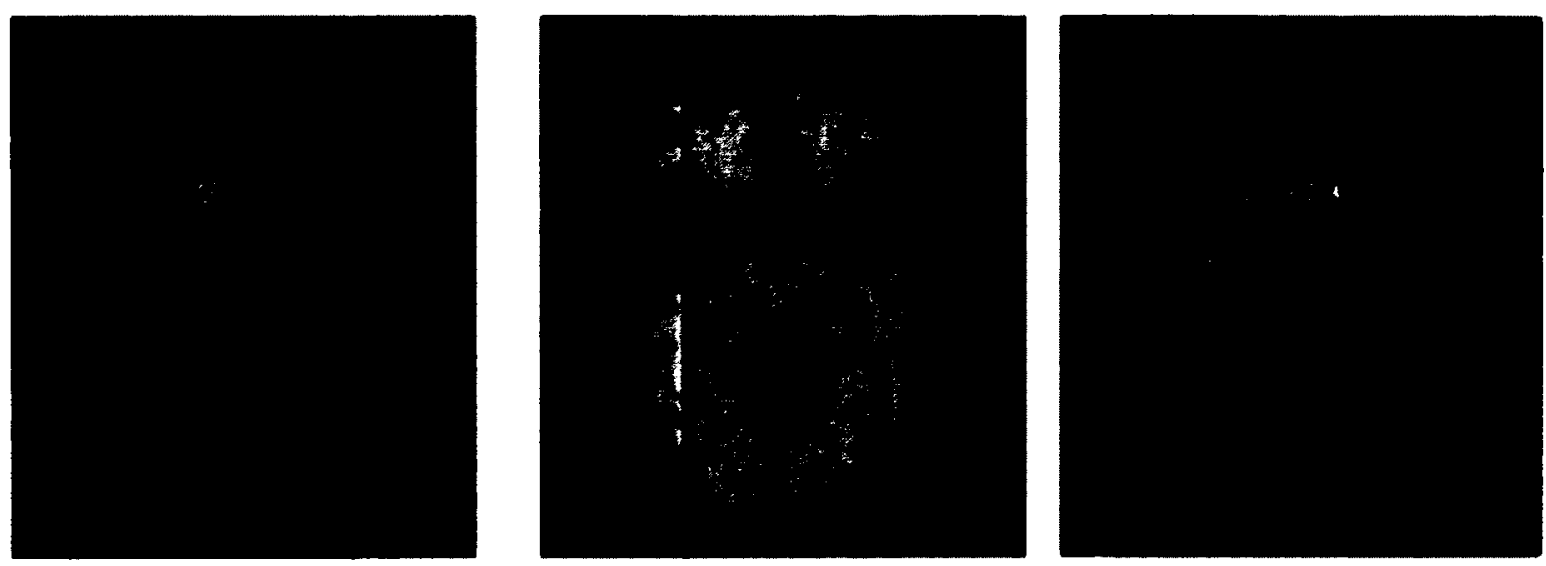

Figure 3.2: Positioning of MRS voxels for perigenual anterior cingulate cortex (left). left. anterior insula (middle), and supragenual anterior (ingulate cortex (right). Each voxel is superimposed on a $T_{1}$-weighted anatomical image from a single subject.

as their ratio to the measured creatine concentration. As a slight interdependence, due to a spectral overlap in their resonances, exists between quantification results for glutamate and glutamine these were quantified together. This combined concentration ratio of glutamate glutamine is referred to henceforth as Glx and is entered into analyses as a ratio to creatine (Cr). Correlation analyses between subject age and Glx Cr levels in each region were carried out (Spearman's rho, two tailed) no relationship between age and $\mathrm{Glx} / \mathrm{Cr}$ concentration was found in any of the MRS regions.

MRS measurements were carried out during the resting state for both methodological and conceptual reasons. The acquisition of MRS data with a suitably high resolution requires a long acquisition time - approximately 12 minutes in this case. Such protracted timescales, with large numbers of encoding steps. make a functional MRS approach (Mangia et al., 2007 ) technically extremely difficult in the context of the task type used, if not impossible. Most importantly; our hypothesis was aimed at the impact of resting state related signal changes in the pgACC on stimulus-induced signal changes in other regions. By searching for correlations of resting state levels of glutamate in the pgACC with stimulus-induced signal changes in the insula we were able to experimentally investigate this hypothesis. It should be noted. however. that the MRS technique does not allow one to distinguish between intraand extra-cellular metabolite pools. Nor does it allow the differentiation of neuronal and 
non-neuronal metabolite concentrations. As such, it is not possible to directly assess the level of transmitter within the synaptic cleft during stimulation using MRS.

\section{Combination of $\mathrm{FMRI}$ and MRS data}

Individuals' measures of $\mathrm{Glx} / \mathrm{Cr}$ concentration in the $\mathrm{pgACC}$ were included in a secondlevel SPM-based regression analysis with the statistical maps of each subject for each of the contrasts of interest. These regression analyses thus identified those brain regions in which the signal changes during the particular condition of interest are related to the level of $\mathrm{Glx} / \mathrm{Cr}$ in the pgACC MRS region. Only those clusters significant after correcting for multiple comparison errors (FWE-correction) were considered.

\subsection{Results}

Results for each stage of the analysis are given. Peak voxel coordinates in MNI-space are given in parenthesis $\left(\begin{array}{lll}x & y & z\end{array}\right)$, along with the related cluster's p-value. Unless otherwise stated, all p-values given are FWE-corrected for multiple comparisons.

\subsubsection{Positive signal changes}

The empathy task evoked positive signal changes (i.e., a task-induced activation) in the $\operatorname{sgACC}\left(-81842, p_{\mathrm{FWE}}<0.001\right)$. This cluster of activation includes the region defined as the sgACC MRS voxel (Figure 3.3 and Supplementary table B.1). This region overlaps with the sgACC region of activity observed in the task-validation meta-analysis (Figure 3.1). No activation in the sgACC was observed during the empathy evaluation task or during the reward task, demonstrating that the activation in this region is task-specific.

Activation in the left anterior insula was observed $\left(-3416-2, p_{\mathrm{FWE}}=0.005\right)$, in line with previous studies of empathy (Singer et al., 2009). No significant activation was seen in the right anterior insula. The empathy task was also seen to induce activity in, amongst other regions, the bilateral amygdala, extending to the ventral striatum $\left(-18-18-20, p_{\mathrm{FWE}}=\right.$ 
$\left.0.014 ; 22-6-16, p_{\mathrm{FWE}}=0.001\right)$, again consistent with previous studies of empathy (Ruby and Decety, 2004)(see Supplementary table B.1 for full activation details).

\subsubsection{Negative signal changes}

As described above, the pgACC has been consistently observed to display a stimulusinduced negative BOLD response, and so the presence of empathy-task induced deactivation in this region was investigated. The contrast [fixation $>$ empathy] does reveal such deactivation from rest in the pgACC as a whole, with the peak voxel of this cluster $(-6408$, $\left.p_{\text {FwE }}<0.001\right)$ lying within the specific portion of this region defined as the pgACC MRS voxel (See Figure 3.3 and Supplementary table B.2). A similar region of deactivation was observed in the pgACC in response to the empathy evaluation task, as well as the reward task. This demonstrates that the deactivation in this region is task non-specific.

Other regions of what has been described as the default mode network (Raichle et al., 2001), which includes that pgACC, were also seen to display a task-induced deactivation during the empathy task, specifically a cluster containing the posterior cingulate cortex (PCC) and precuneus $\left(-10-6212, p_{\mathrm{FWE}}<0.001\right)$ (Figure 3.3 and Supplementary table B.2).

\subsubsection{Inter-regional connectivity}

Connectivity between the pgACC and sgACC was tested using psychophysiological interaction (PPI) analyses. Taking the pgACC MRS voxel as the seed region, functional connectivity from this region with the sgACC was observed $\left(02232, p_{\mathrm{FWE}}=0.004\right.$; Supplementary table B.3). The reciprocal connectivity, investigated using the sgACC MRS voxel as seed region, between the sgACC and pgACC was not observed (Supplementary table B.4). Hence, only when taking the pgACC as seed was a relationship with the signal changes in the sgACC observed, with this not being the case in the reverse direction: this indicates the uni-directional connectivity from the $\mathrm{pgACC}$ to the sgACC during empathy.

The pgACC also displayed functional connectivity with, amongst other regions, the right

hippocampus, extending to the amygdala $(22-30-6, p<0.001)$, the bilateral posterior 


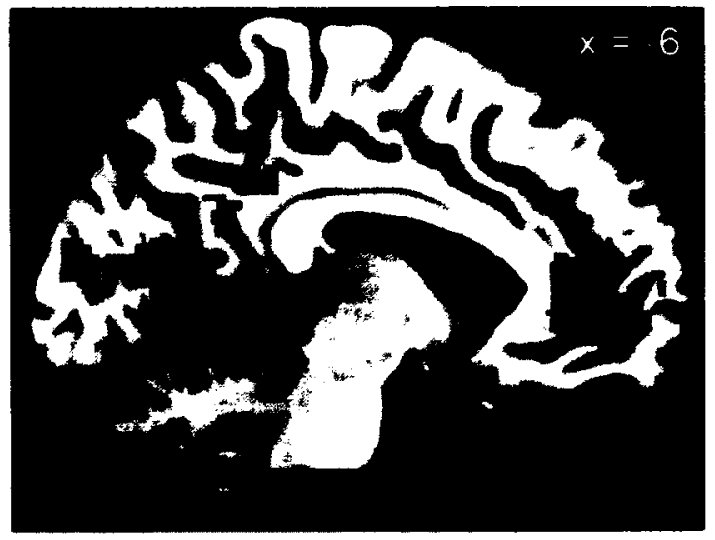

fixation > empathy

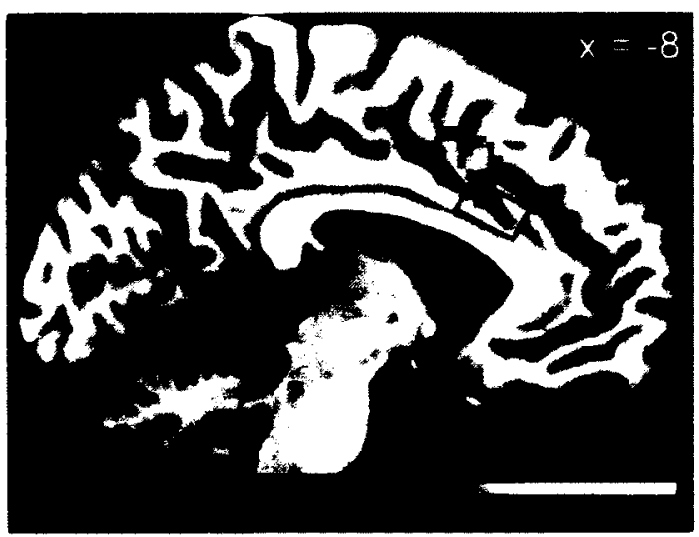

empathy > view smoothed
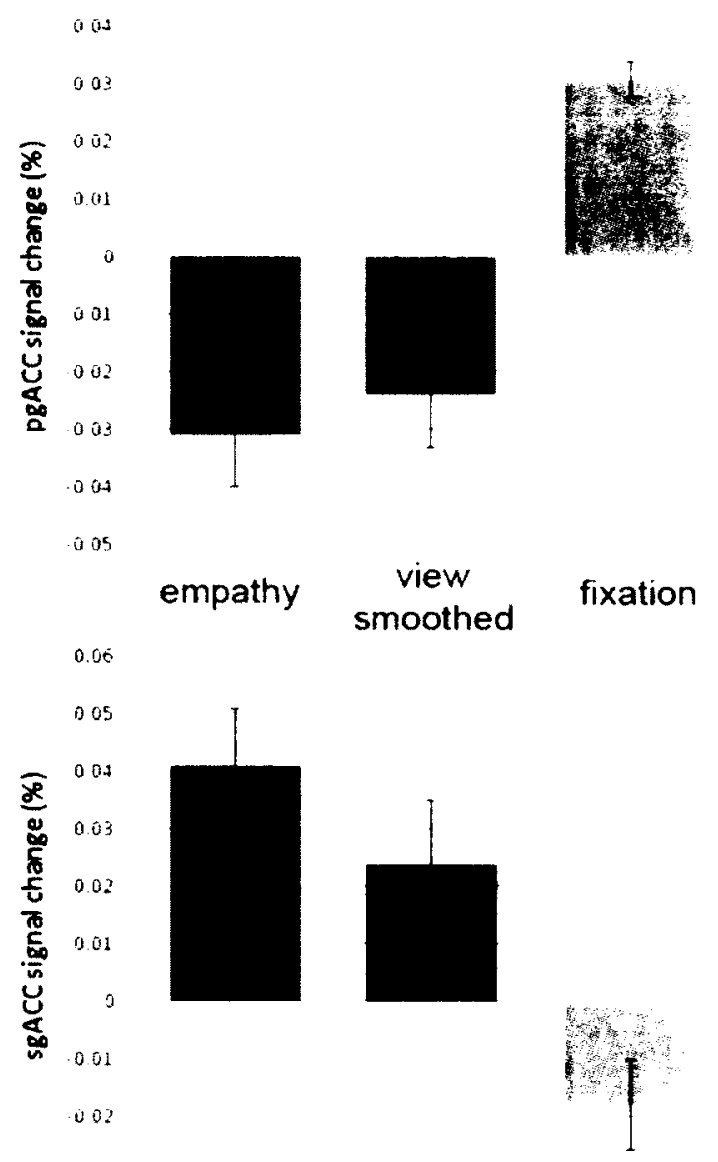

Figure 3.3: Areas of deactivation from rest in response to the empathy task are shown (contrast [fixation empathy]), along with activations in response to the empathy task (contrast [empathy smoothed]). Mean percent signal changes are shown for the viewing of emotional pictures, the viewing of smoothed pictures, and the fixation period in the pgACC (red box) and sg.ACC (green box) MRS voxels. Mean percentage signal changes were calculated using the Marsbar toolbox (available at: http://mars-bar sourceforge .net/). Error bars represent SEMI. Images are shown with a threshold of $p_{\text {unc }} \quad 0.005$ for the purpose of illustration. 
cingulate cortex $\left(-12-4232, p_{\mathrm{FWE}}<0.001\right)$, the left thalamus $\left(-12-142, p_{\mathrm{FWE}}=0.001\right)$, and the right thalamus extending to the ventral striatum $\left(10-86, p_{\mathrm{FWE}}<0.001\right)$ (see Supplementary table B.3 for full details).

Functional connectivity between the sgACC and, amongst other regions, the bilateral posterior cingulate cortex $\left(-6022, p_{\mathrm{FWE}}<0.001\right)$ was observed (see Supplementary table B.4 for full details). This cluster runs posterior from the sgACC, via the mid-cingulate, following approximately the line of the corpus callosum.

The specificity of the functional connectivity between the pgACC and sgACC for empathy was tested by carrying out two additional PPI analyses using the pgACC MRS voxel as seed region. Neither the PPI analysis using emotional evaluation as the psychological factor, nor that using reward, showed any connectivity between the pgACC and the sgACC.

\subsubsection{Resting state $\mathrm{Glx}$ and regional signal changes}

SPM-based regression analyses of the BOLD response to the empathy task and the $\mathrm{Glx} / \mathrm{Cr}$ concentration in both the pgACC and $\mathrm{sgACC}$ were carried out. These analyses identified in which regions of the brain the neural response to the task is related to the level of $\mathrm{Glx} / \mathrm{Cr}$ in the particular MRS voxel.

A relationship between the concentration of $\mathrm{Glx} / \mathrm{Cr}$ in the $\mathrm{pgACC}$ and the empathy-task induced BOLD response was observed in the $\operatorname{sgACC}\left(-121236, p_{\mathrm{FWE}}<0.001\right)$, with this cluster overlapping with the sgACC MRS voxel (Figure 3.4). In addition, there was a relationship between the concentration of $\mathrm{Glx} / \mathrm{Cr}$ in the pgACC and the neural response in the left precuneus $\left(-26-6648, p_{\mathrm{FWE}}=0.001\right)$, as well as with the response in the bilateral amygdala $\left(-26-2-10, p_{\mathrm{FWE}}<0.001 ; 306-14, p_{\mathrm{FWF}}<0.001\right)$ (Table 3.1$)$.

No relationship was observed between the concentration of $\mathrm{Glx} / \mathrm{Cr}$ in the sgACC and taskinduced BOLD responses in the rest of the brain when results were corrected for multiple comparison errors. An uncorrected correlation between the sgACC Glx/Cr level and the BOLD response in the right superior frontal gyrus (24 $4426, \mathrm{p}=0.006$ unc.), right fusiform gyrus (44 -60 -16, $p=0.01$ unc.) and left mid-insula $(-408-8, p=0.032$ unc.) was observed, however (Supplementary table B.5). 
Negative signal changes (pgACC)

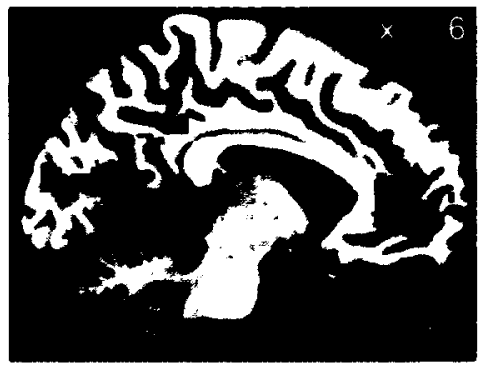

fixation > empathy

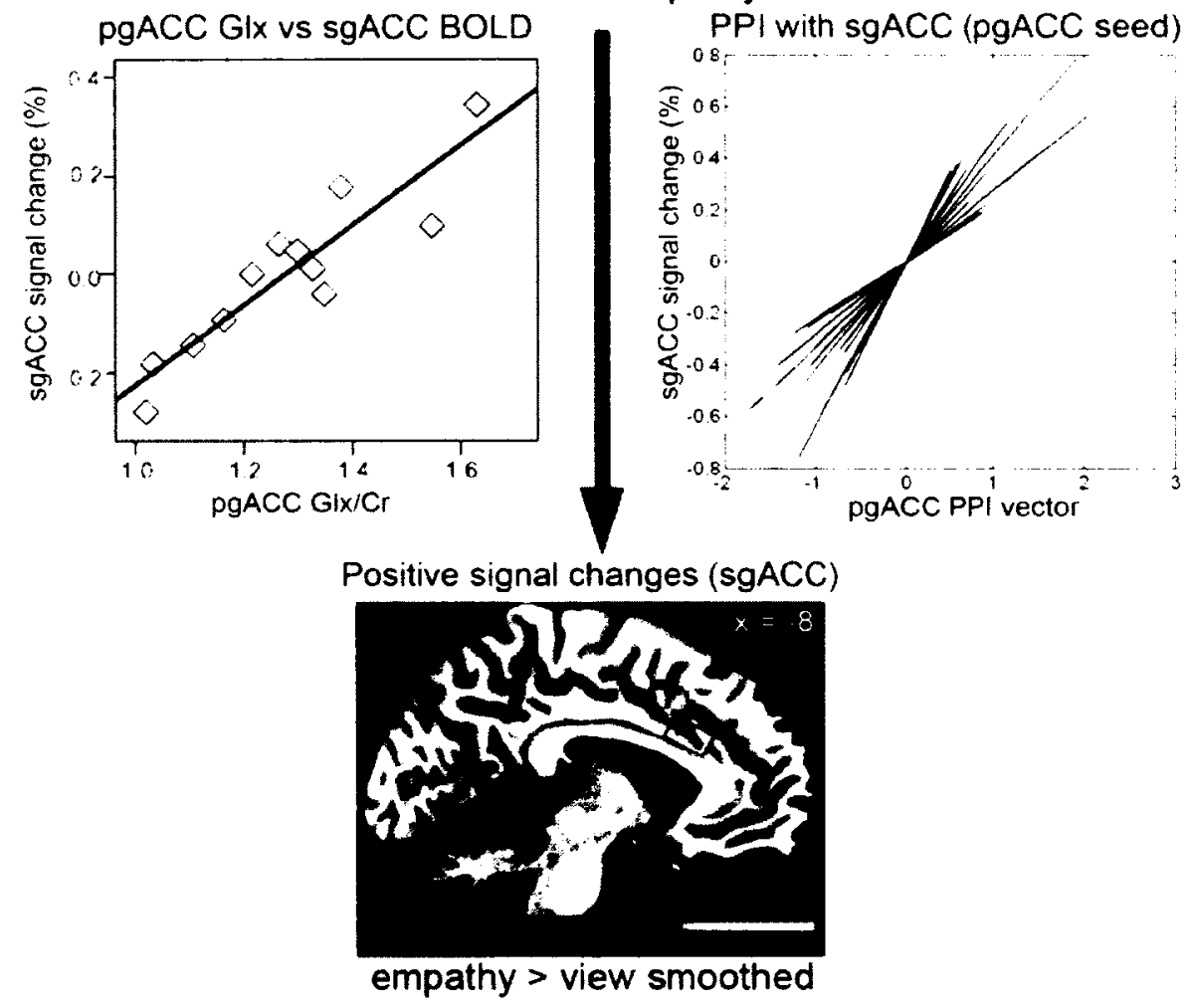

Figure 3.4: Unidirectional connectivity between negative signal changes in the pgACC MRS region (red box) and positive signal changes in the sgACC MRS region (green box) during the empathy task was demonstrated using PPI analyses. A relationship between the BOLD response during the same task in the sgACC and the level of glutamate in the pgACC was demonstrated using regression analyses. A plot of the regression between the mean $\operatorname{sgACC} B O L D$ response and pgACC glutamate at the peak regression voxel within the sgACC is shomn. A combined plot of the PPI regression results for each individual subject obtained from their first level PPI analysis at the peak voxel within the $\mathrm{sgACC}$ (from the group level analysis) is also shown. These results demonstrate glutamatergically mediated communication between the task-negative pgACC and task-positive sgACC during empathy. 


\begin{tabular}{|c|c|c|c|c|}
\hline Region & & $x, y, z$ & voxels & Z-score \\
\hline \multirow[t]{6}{*}{ Amygdala/putamen } & $\mathrm{L}$ & $-26-2-10$ & 829 & 4.54 \\
\hline & & $-286-10$ & & \\
\hline & & $-35-2-2$ & & \\
\hline & $\mathrm{R}$ & $306-14$ & 766 & 4.26 \\
\hline & & $30-4-8$ & & \\
\hline & & $32-12-8$ & & \\
\hline \multirow[t]{3}{*}{$\mathrm{sgACC} / \mathrm{MCC}$} & $\mathrm{L} / \mathrm{R}$ & -121236 & 1034 & 4.35 \\
\hline & & -8230 & & \\
\hline & & 82622 & & \\
\hline Superior temporal gyrus & $\mathrm{R}$ & 6066 & 275 & 4.25 \\
\hline \multirow[t]{2}{*}{ Precuneus } & $\mathrm{L}$ & $-26-6648$ & 295 & 3.92 \\
\hline & & $-42-6438$ & & \\
\hline \multirow[t]{2}{*}{ Supramarginal gyrus } & $\mathrm{R}$ & $68-1824$ & 249 & 3.73 \\
\hline & & $56-1430$ & & \\
\hline
\end{tabular}

Table 3.1: Regions showing correlation between BOLD response during empathy task (contrast (empathy $>$ view smoothed]) and Glx/Cr levels in pgACC. Peaks and cluster sub-peaks are shown. Coordinates are given in MNI space. Cluster $p_{\mathrm{FWE}}<$ 0.05. (sgACC $=$ supragenual anterior cingulate cortex; $\mathrm{MCC}=$ mid cingulate cortex) 
To test the specificity of the relationship between the concentration of $\mathrm{Glx} / \mathrm{Cr}$ in the $\mathrm{pg} \mathrm{ACC}$ and neural responses in the sgACC during empathy, regression analyses of $\mathrm{pgACC} \mathrm{Glx} / \mathrm{Cr}$ with the BOLD response during both the empathy evaluation task and the reward task were carried out. Neither regression showed any relationship between $\mathrm{pg} A \mathrm{AC} \mathrm{Glx} / \mathrm{Cr}$ and the neural response in the sgACC during the respective conditions. In addition, regression analyses of the neural response during the empathy, empathy evaluation and reward tasks with the concentration of $\mathrm{Glx} / \mathrm{Cr}$ in the left anterior insula were carried out. No areas within the cingulate cortex were identified by these analyses.

\subsection{Discussion}

The involvement of glutamate in the interaction between resting state related activity in a task-negative region and stimulus-induced activity in a task-positive region is reported here. This provides insight into the neuronal and biochemical mechanisms by means of which the resting state activity of the brain potentially impacts upon subsequent stimulus-induced activity.

The first main finding of the study concerns the relationship between signal changes in task-negative and task-positive regions. Positive BOLD responses (activations) in the taskpositive sgACC were reported, along with a negative BOLD response (deactivation) when compared to rest in the task-negative pgACC. These negative BOLD responses in the $\mathrm{pgACC}$ were seen to display functional connectivity with the positive BOLD responses in the sgACC. The functional connectivity between the pgACC and sgACC was shown to be uni-directional and specific to the empathy task, as compared to control empathy-evaluation and reward tasks.

The second main finding of the study concerns apparent glutamatergic modulation of reststimulus interaction between the two target regions. Glutamate concentrations in the two regions of interest within the $\mathrm{ACC}$, along with a control region in the left anterior insula, were measured using MRS (as a combined measure of glutamate and glutamine). It was found that signal changes during the empathy task in the sgACC were related to the level of glutamate in the pgACC. No reciprocal relationship between the signal changes in the

pgACC and the level of glutamate in the sgACC was found. Similarly, no relationship was 
found between signal changes in either the pgACC or sgACC and the level of glutamate in the left anterior insula, suggesting that the relationship observed with the $\mathrm{pgACC}$ was not due to a global effect of glutamate concentrations in the brain.

The observed interaction between the pgACC and sgACC during empathy can be interpreted as an instance of task-induced deactivation in one region interacting with a separate area of task-induced activation. In agreement with previous findings (e.g., Grimm et al., 2009, Northoff et al., 2007, Raichle et al., 2001, Simpson et al., 2001), the task-induced deactivation in the $\mathrm{pgACC}$ was seen to not be task-specific, with a similar extent of NBR seen in response to empathy, empathy evaluation and reward. In contrast, the task-induced activation in the sgACC was demonstrated to be specific to empathy, with no activation in this region in response to the two control tasks.

A relationship between task-negative and task-positive regions has been described in the resting state (Fox et al., 2005, Hampson et al., 2010), whilst resting connectivity within the task-negative DMN has been shown to affect task induced BOLD responses in task-positive regions (Mennes et al., 2010). The current study thus extends these findings by demonstrating that negative BOLD responses in the DMN are connected to task-specific BOLD responses in other brain regions. More specifically, a relationship between the positive and negative signal changes in the two regions of interest was demonstrated through the observed functional connectivity. This suggests that task-induced activation in the sgACC is related to the degree of task-induced deactivation in the pgACC. The uni-directionality of the relationship further supports the hypothesis that the level of resting state activity in the pgACC impacts upon, and potentially modulates, stimulus-induced activity in the sgACC. Such a situation would lend support to the concept of rest-stimulus interaction, where resting state and stimulus-induced activity are functionally interrelated (Northoff et al., 2010b).

Both subregions of the ACC studied have rich glutamatergic innervation (Bozkurt et al., 2005, Palomero-Gallagher et al., 2009), and so this neurotransmitter system, the main excitatory neurotransmitter system and important in cortico-cortical communication, was deemed likely to play a role in communication between the two regions. This was confirmed in this study through the combination of functional connectivity analyses and the measuring of glutamate using MRS. That the relationship between glutamate concentration and interregional signal changes was found only in the case of pgACC glutamate levels further 
supports the uni-directional nature of the communication between the pgACC and sgACC described here.

Previous studies have demonstrated the involvement of GABA in task-induced deactivation in both task-positive and task-negative regions (Muthukumaraswamy et al., 2009, Northoff et al., 2007). These findings, however, leave open the question as to how interactions between task-negative and task-positive regions are mediated. The implication of glutamate in the interaction between the task-negative $\mathrm{pgACC}$ and task-positive sgACC in the current study thus provides initial evidence for a role for this neurotransmitter system in reststimulus interaction.

One may also speculate as to the relationship between glutamate, an excitatory neurotransmitter, and deactivation from rest in the pgACC. The high level of resting state in this region, and by extension other task-negative regions, may be an outcome of high ongoing glutamatergic activity in these regions. A reduction in this glutamatergic activity, or a counteraction of it by an inhibitory neurotransmitter such as GABA, may then lead to the observed task-induced deactivations. The level of tonic glutamatergic activity would thus determine the level of resting state activity, and from this, would indirectly determine the potential for deactivation during a given task. This hypothesis remains speculative at present and requires further investigation.

In addition to their significance to our understanding of the interaction between tasknegative and task-positive regions, this study's findings may be of some interest in the context of empathy. Both of the main findings of the study were specific to the empathy task employed, as distinct from the control empathy evaluation and reward tasks. This is in accordance with the reported central role of the sgACC in empathy (Prehn-Kristensen et al., 2009, Singer and Lamm, 2009). The current findings extend these previous observations by showing that task-induced activity in the sgACC during empathy interacts with task-induced deactivation in the pgACC, providing evidence that such deactivation in the pgACC may play a similarly important role in empathy processing. Finally, it was demonstrated that the interaction between the pgACC and sgACC during empathy was related to resting-state glutamate levels in the pgACC. This lends further support to the observed glutamatergic modulation of emotional and neural function related to the pgACC and sgACC by glutamatergic substances such as ketamine (Etkin and Wager, 2007, Salvadore et al., 2009,2010 ). These observations in the context of empathy must, of course, be treated as 
speculative and need to be investigated further.

The observation of glutamatergic modulation of emotional processing as in empathy may also be relevant in psychiatric disorders. Changes in resting state activity and connectivity have been linked to a number of psychiatric disorders, including schizophrenia and major depressive disorder (Broyd et al., 2009, Buckner et al., 2008, Garrity et al., 2007, Greicius et al., 2007). Particularly in depression, abnormally elevated resting state activity and glutamatergic abnormalities have been observed in the pgACC (Alcaro et al., 2010, Buckner et al., 2008, Walter et al., 2009). This study's findings thus raises questions as to how this abnormally elevated resting state activity in the pgACC relates to glutamatergic function, as well as to the modulation of stimulus-induced activity in the $\mathrm{sg} A C C$, a region that has been observed to show decreased activity during emotional and cognitive tasks in depression (Anand et al., 2005, Hooley et al., 2009). Hence, it may be of interest in the future to investigate depressed patients using a similar design to that reported here.

Some limitations of the present study should be considered. Resting state activity in the pgACC is inferred indirectly via the occurrence of a negative BOLD response elicited by stimuli, while glutamate levels were measured directly in the resting state. Though previous studies pursued a similar approach (Northoff et al., 2007), future studies may wish to take a more direct approach to measuring resting state activity itself, relating this then to glutamate levels. Measurements of changes in human glutamate concentration during stimulus-induced activity are also needed to further confirm the assumption that the direct and indirect measurements can safely be compared. In a similar vein, it should be noted that glutamate was measured here as a single value along with its precursor glutamine, as represented by the designation Glx. Replication using a more sensitive MRS analysis that allows for a distinction between glutamate and glutamine would be desirable. It should also be mentioned that the focus of the analysis was on glutamate, and as such the role of GABA was not investigated. The role of GABA in the context of rest-stimulus interactions thus needs to be investigated in further detail in the future. The fixation cross period taken as a representative resting state condition in the study was rather short, and so can only be taken to be an approximation of a true resting state; future studies may thus wish to replicate these findings with longer resting state periods. Finally, the current sample size is not large, and so results should be treated as preliminary. Replication with a larger sample size is required. 
In summary, the present study demonstrates an interaction between resting state dependant responses in the task-negative pgACC and task-induced activations in the task-positive sgACC. Connectivity between the regions was further shown to be related to glutamate concentrations in the pgACC specifically. These results provide preliminary evidence that glutamate plays a central role in rest-stimulus interaction in the human brain.

\subsection{Acknowledgements}

Many thanks to M. de Greck for his invaluable help with the study design and analysis, D. Hayes for his useful comments, and E. Stockum and C. Ulrich for their assistance with the recruitment of subjects and conducting of scanning sessions. Thanks also to the staff at the Department of Neurology at the Universitätsklinikum Magdeburg for their skillful assistance. The work was supported by grants to G.N. from the Hope of Depression Research Foundation and German Research Foundation (DFG/SFB 776 A6). 


\section{Chapter 4}

\section{Glutamate and intra-regional activity}

In the previous chapter, it was demonstrated that the level of Glx/Cr in the pgACC correlated positively with the activity contrast between the viewing of emotional faces versus the viewing of smoothed faces ([empathy $>$ smoothed]) in the sgACC. Thus, with a greater concentration of $\mathrm{Glx} / \mathrm{Cr}$ in the $\mathrm{pgACC}$, there was a greater activity difference between the control smoothed faces condition and the emotional faces condition. In addition, it was shown that there was a functional coupling between the resting-state related activity in the pgACC (as measured during the fixation cross period) and the stimulus-induced activity in the $\operatorname{sgACC}$.

What was not shown, however, was a relationship between the level of $\mathrm{Glx} / \mathrm{Cr}$ in the $\mathrm{pgACC}$ and measures of activity within the same region. This raises the question as to how glutamatergic measures are related to intra-regional activity in the pgACC, as opposed to inter-regional connections (in the context of the empathy task employed, at least). To investigate this question, the data was reanalysed in order to obtain measures of the activity within the pgACC during each of the three conditions, as opposed to the difference in activity between the conditions (as is accessed by the contrasts employed in the paper). The values for the individual conditions were then compared to the Glx/Cr measures from the region.

The same data and basic analysis approach were used as in the previous chapter. Briefly, an emotional face viewing task was employed in fMRI, along with MRS measurements from the pgACC. The contrast [fixation > view empathy] was made to demonstrate a negative BOLD response (NBR) to the empathy task in the pgACC. Signal changes for each of the conditions of interest (fixation, view empathy, evaluate empathy) were then extracted from the region corresponding to the pgACC MRS voxel (box, $20 \times 10 \times 20 \mathrm{~mm}^{3}$ ) 
for each subject using the Marsbar toolbox (http://marsbar. sourceforge net/). These signal changes were then correlated with the level of $\mathrm{Glx} / \mathrm{Cr}$ in the pgACC (Spearman, two-tailed) to identify those conditions that showed a relationship with this measure.

The contrast [fixation > view empathy] demonstrated a NBR in the pgACC in response to the empathy task (Figure 4.1A). NBR was also seen in the posterior cingulate cortex. The same response was seen in the [fixation > evaluate empathy] contrast. No correlation was seen between the level of Glx/Cr and signal changes during either the empathy viewing ( $R$ $=0.07, p>0.9)$ or empathy evaluation $(\mathrm{R}=-0.32, p>0.3)$ conditions (Figure 4.1B). In contrast, a correlation between baseline activity (as represented by the fixation condition) and the level of $\mathrm{Glx} / \mathrm{Cr}$ in the pgACC was seen $(\mathrm{R}=0.62, p=0.03)$.

To provide information about the localisation within the MRS voxel of the correlation between signal changes and $\mathrm{Glx} / \mathrm{Cr}$ levels, whole-brain regression analyses were carried out (Figure 4.1C). The regression of signal changes during the empathy task ([fixation > empathy]) with Glx/Cr revealed a significant cluster within the MRS voxel (10 $420, p_{\text {FWE }}$ $=0.03)$. This cluster extends laterally from the pgACC voxel $(\mathrm{k}=18)$. A second cluster $(-$ $1038-12, p_{\mathrm{FWE}}=0.016$ ) was observed in the opposing hemisphere that overlaps to a small degree with the MRS voxel $(k=78)$. A significant cluster was also identified within the $\mathrm{pgACC}$ MRS voxel in the regression of [fixation > evaluate empathy] with $\mathrm{pgACC}$ Glx $/ \mathrm{Cr}$ levels $\left(10422, p_{\mathrm{FWE}}=0.024\right)$. This cluster extends laterally in a similar manner to the cluster identified in the [fixation $>$ empathy] regression $(\mathrm{k}=24)$.

In summary, the empathy task induced deactivation in the pgACC, as well as in the PCC. A positive correlation was seen between $\mathrm{pgACC} \mathrm{Glx} / \mathrm{Cr}$ and the percent signal change value during the fixation period. This correlation was specific to the fixation period, with no relationship seen with the percent signal change values for either the view empathy or evaluate empathy conditions. In addition, a positive correlation between the contrast [fixation > view empathy] and pgACC Glx/Cr was seen within the MRS voxel.

As described in the previous chapter, the observed task-induced NBR would be expected, given that the identified regions lie within the so-called DMN and as such generally show high levels of activity at rest (operationalised here as 'fixation'), along with decreased activity when an attention requiring task is performed (Grimm et al., 2009, Gusnard and Raichle, 2001, Northoff et al., 2010b). The specific correlation between the pgACC fixation 

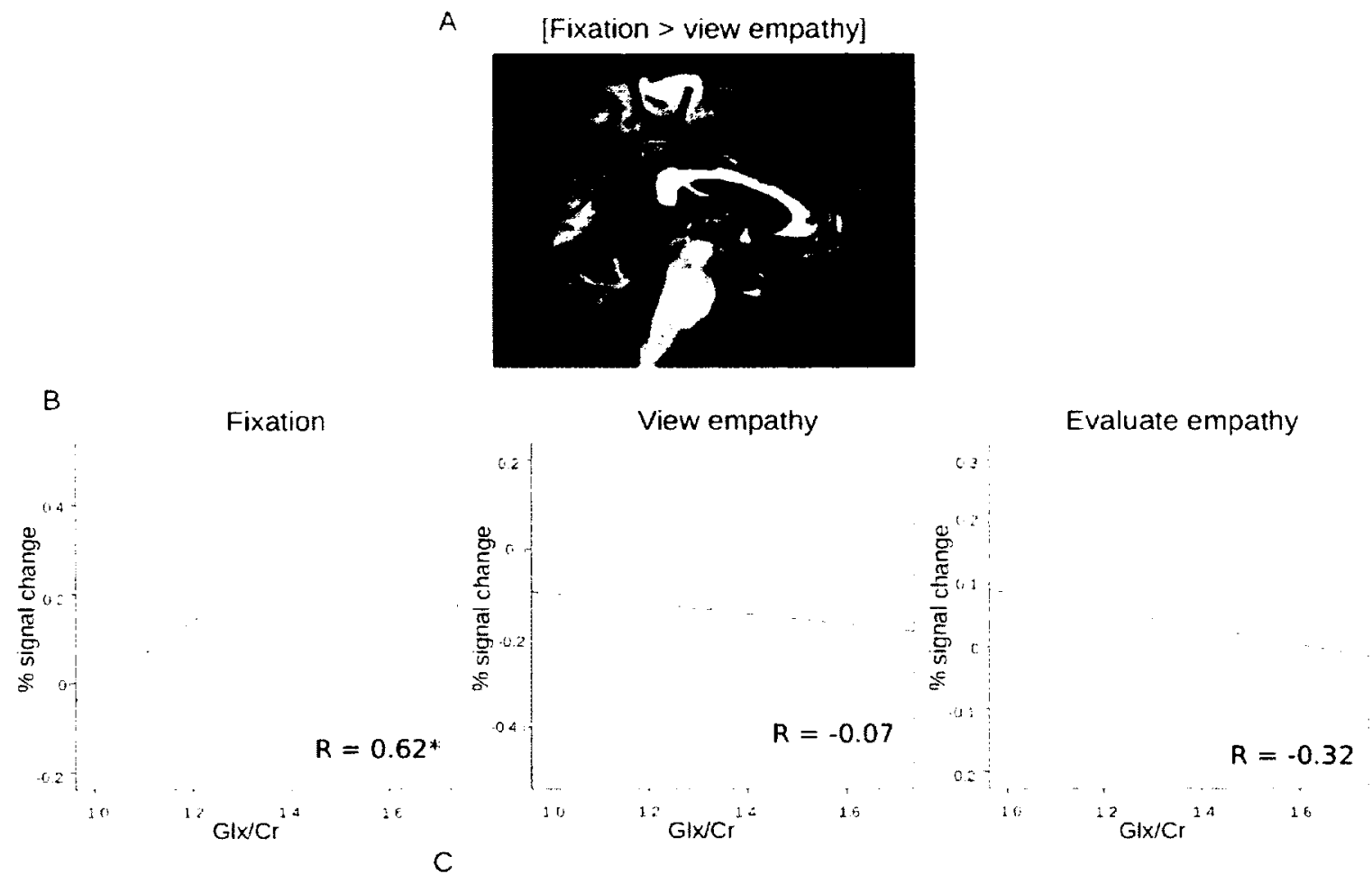

C

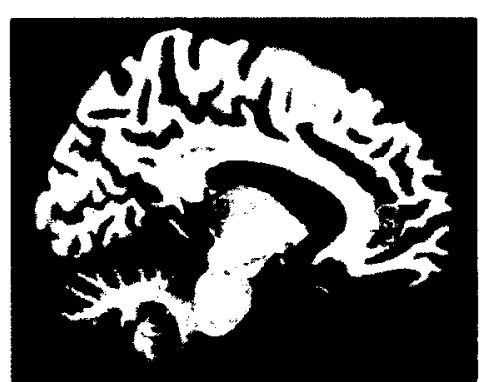

[fixation > view empathy] - Glx

Figure 4.1: (A) Deactivations induced by the empathy task are shown along with the pgACC MRS region in green. (B) Correlations between fixation, empathy viewing, and empathy exaluation signal changes and pgACC Glx Cr. * indicates p 0.05 . (C) Voxel-wise regression of pgACC Glx $\mathrm{Cr}$ and empathy-induced deactivations showing a significant cluster within the MRS region. pgACC $\mathrm{Glx} / \mathrm{Cr}$ is correlated with fixation only and not empathy-related activity. 
percent signal change and $\mathrm{Glx} / \mathrm{Cr}$ within the same region then suggests that the level of activity from which such NBR occurs is influenced by the concentrate of $\mathrm{Glx} / \mathrm{Cr}$ within the region. In addition, the fixation-related contrast correlation with $\mathrm{Glx} / \mathrm{Cr}$ ([fixation > view empathy] vs pgACC $\mathrm{Glx} / \mathrm{Cr}$ ) points towards this $\mathrm{Glx} / \mathrm{Cr}$ related resting activity level influencing the degree of intra-regional response to the empathy task. More specifically, a higher $\mathrm{Glx} / \mathrm{Cr}$ concentration correlates with a higher level of activity during fixation, coupled with a larger degree of NBR in response to the task.

[Note that the analysis described above is a confirmation of a similar analysis published by the author and colleagues as Enzi et al. (2012).]

The final interpretation of these results requires some caution, however, as the fMRI approach adopted does not give an absolute measure of neural activity. Instead, the activity level is indirectly inferred, firstly via the BOLD response, and then secondly from the outcome of the SPM analysis. This second inference is the one of greater relevance here, as the "percent signal change" is the result of a series of calculations on the beta values produced in the SPM linear regression, rather than a direct measure of brain activity or activity changes. A number of factors can thus influence the "percent signal change" values, including the degree of noise or signal-related variance in the data (affecting the fit to the model), or the particular length of events in the model. The event lengths here are identical across subjects, and so this factor can be discounted; the variance in the data is an interesting issue in itself, however, that is not amenable to investigation using the current approach but which may contain information about neural function (Garrett et al., 2010, 2011). The "percent signal change" value could then represent either a true increase in activity level, or alternatively differential resting variance across subjects that is reflected in different signal change values for the fixation period (or, most likely, a combination of these plus other factors). In order to provide a more full insight into the relationship between glutamate and intra-regional activity, then, more sophisticated approaches are required that give information as to the dynamic properties of the signals themselves, rather than their relationship to any particular observer-imposed model (Northoff et al., 2010a) or to patterns of activity in other regions (Smith et al., 2011). This is the aim of the following chapter, where specific dynamic measures are applied to task-independent activity timecourses and combined with glutamate MRS.

Two different dynamic measures are employed in Chapter 5 - standard deviation (SD) and 


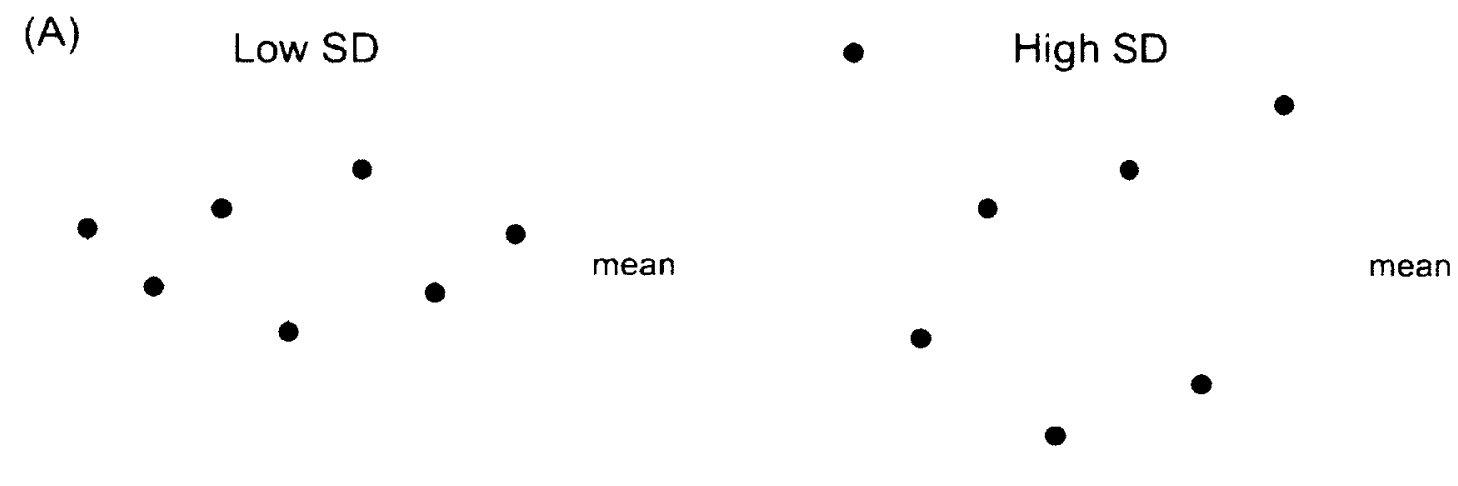

(B)

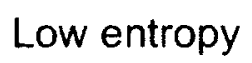

High entropy
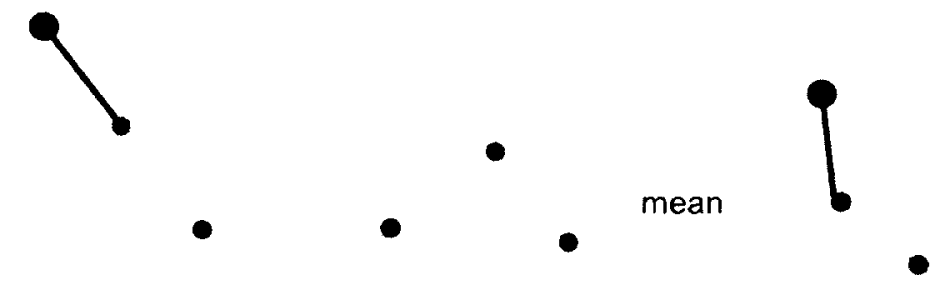

- mean

Figure 4.2: (A) Illustration of a set of values that hare a low (left) and high (right) SD, where the latter set of values vary more around the mean. (B) Timeseries that have low (left) and high (right) entropy. In the low entropy timeseries, regular patterns can be seen, meaning that sets of values (green) are predictable from the order of values preceeding them (red). In the high entropy series, no such structure exists, making proceeding values hard to predict based on any one set (red). 
entropy. The first of these is a widely used measure of how much the individual members of a set of values vary in relation to their mean; a set of data with a large SD will vary a lot in relation to the mean across them, whilst a set of data with a small SD will be clustered more closely around the mean value (i.e., will be less variable; see Figure $4.2 \mathrm{~A}$ ). It should be noted that, strictly speaking, SD is a static rather than dynamic measure, as the order in which values appear in the set is not relevant to the SD; however, it can be classed as a dynamic measure here as it applies to the full activity timeseries per se, rather than to a fitting of a timeseries with a particular model. In contrast, entropy represents a more true dynamic measure as it takes into account the way that values relate to each other over time. Entropy can be described as a measure of how easy it is to predict proceeding values in a series given the distribution of the values that have come before. A timeseries that consisted of all the same values would thus have zero entropy (as it is possible to predict with absolute certainty the next value given the ones prior), whilst random noise would have high entropy (as it is, by definition, very uncertain what value comes next in a random series). All timeseries will lie between these extremes, entropy increasing as the irregularity of their structures increase (see Figure $4.2 \mathrm{~B}$ for an illustration). 


\section{Chapter 5}

\section{Glutamate related entropy in the mPFC alters between eyes-open and eyes-closed rest}

\subsection{Abstract}

Studies of ongoing activity in the brain have provided insight into its organisation and functioning. However, fewer studies have investigated the dynamic properties of brain activity in humans in vivo. We thus used a combination of EEG and fMRI scanning in eyesopen and eyes-closed rest to quantify activity dynamics and the effect of contextual shifts (i.e., from eyes-closed to eyes-open) on them. Standard deviation and sample entropy were used as complementary linear and non-linear measures. It was found that both measures altered between eyes-open and eyes-closed rest, but that these changes were in different regions of the brain. In addition, MRS was used to measure glutamate concentrations in the medial prefrontal cortex (mPFC). It was found that $\mathrm{mPFC}$ glutamate correlated with entropy in the same region during eyes-closed rest, but not eyes-open. These results provide insights into the dynamical organisation of the brain and its biochemical underpinnings.

\subsection{Introduction}

The brain is a dynamic system in which activity produced by stimuli external to it interact with ongoing internal activity. Whilst this ongoing internal activity has for a long time been treated as uninteresting noise, it is becoming increasingly apparent that this 'noise' is important to brain function (Deco et al., 2011, Northoff et al., 2010b, Raichle, 2009). For example, the interaction between ongoing activity and particular external stimuli has been 
shown to determine the physical and psychological response to said stimuli (Sadaghiani et al., 2010). Many studies have investigated the manner in which ongoing activity is structured on an inter-regional level, revealing detailed aspects of intrinsic brain networks (Hutchison and Everling, 2012). Similarly, a growing body of literature has investigated the potential underlying sources of ongoing activity and its structure (Deco et al., 2011, Deco and Jirsa, 2012). Fewer studies, however, have focused on the intra-regional properties of ongoing activity, leaving open questions as to the intrinsic dynamics of such activity.

In the context of in vivo human brain activity, two of the measures for investigating timecourse dynamics that have been adopted are standard deviation (SD) (Garrett et al., 2010) and entropy (Costa et al., 2005, Richman and Moorman, 2000). Although both measures generally give an estimation of complexity, entropy differs in respect to SD in that the measure takes into the temporal order of the signal being investigated. Alternately, SD is determined by timepoint values alone, and is independent of the order in which they occur. This means that two signals with the same SD can have differing entropies and thus leaves open the possibility that these two measures, when applied to neural activity, may access somewhat different aspects of brain dynamics. To date, SD has mostly been used in the context of fMRI. Such studies have revealed that signal variability is task and region specific, and that a larger degree of neural activity variability is typically correlated with an improved performance in a range of tasks (Garrett et al., 2010, McIntosh et al., 2008). Entropy, in contrast, has mainly been used in the context of EEG and MEG (Abásolo et al., 2006, ?). Results from such studies have, on the whole, supported those from SD analyses, showing similar relationships between entropy and task performance. The task-sensitivity of each of the measures highlights their sensitivity to situational context, raising the question as to the contextual sensitivity of ongoing (i.e., task-independent) activity dynamics. To investigate this, the eyes open and eyes closed resting-state provides a convenient and simple contextual modulation. Previous studies have shown changes in resting-state brain activity between these two states (Duncan and Northoff, 2012, Hüfner et al., 2009, Yan et al., 2009), whilst the change itself represents a minor undertaking for the participant and so minimises task-effects.

As well as the dynamics of ongoing activity, the biochemistry underlying it is of key interest. Simulation studies have suggested that regional excitation/inhibition balance may play a central role, as manifest through glutamatergic and GABAergic activity (Deco and Jirsa, 2012). This idea is supported by human imaging studies that have linked rest-related 
activity to regional concentrations of these transmitters. For example, resting activity levels and rest-related signal changes are associated with glutamate levels in the mPFC (Duncan et al., 2011, Enzi et al., 2012). Similarly, rest-related signal changes have been found to correlate with GABA concentrations in the same region (Northoff et al., 2007). The link between transmitter levels and signal dynamics remains unclear, however.

Based on the above background, the current study sought to approach a number of questions: Firstly, whether or not the complexity of the neural signal altered between the eyes-open and eyes-closed conditions, taking these as two distinct sensory contexts and using SD and entropy as dynamic metrics; secondly, whether or not entropy values from fMRI data follow similar patterns to those obtained with EEG; thirdly, do entropy and SD measure different aspects of the neural signal, as measured in fMRI; and finally, how might measures of signal complexity relate to neural biochemistry. To approach these questions, we combined an existing resting-state EEG dataset with resting-state fMRI and MRS from a separate group of participants. Resting data were acquired in both the EO and EC condition, allowing a typification of ongoing signal dynamics in both conditions and the identification of any differences. SD and entropy were additionally linked to regional transmitter concentrations through correlation with the MRS measures.

\subsection{Methods}

\subsubsection{Dynamic measures}

The measures of signal dynamics used were SD and sample entropy. The first of these methods quantifies the degree to which individual timepoints differ in value from the mean across the whole timecourse. A timecourse with a greater SD thus displays a wider range of potential values than one in which the SD is lower, and can be linked to a more dynamic signal (Garrett et al., 2011). SD was calculated using the relevant function native to MATLAB (v 7.8.0, The Mathworks Inc.). The second approach, sample entropy, gives an estimation of the randomness of a particular signal, measuring the extent to which regular structures appear within it. This is done by calculating the $\log$ likelihood that a series of points of a length $m$ will also be the same at a particular matching threshold, $r$, at a length of $m+1$ (Richman and Moorman, 2000). An increase in entropy is generally (though not 
always) associated with an increase in complexity (Costa et al., 2005).

Sample entropy was applied in two different ways: It was firstly calculated at a single timescale (i.e., applied to the raw timeseries) in fMRI and EEG using the SampEn toolbox for MATLAB (http://ww.physionet.org/physiotools/sampen/matlab) (Richman and Moorman, 2000). Secondly, it was calculated as multiscale entropy (Costa et al., 2005), where the original timeseries is iteratively downsampled and the entropy values calculated at each progressively more coarse timescale (scale 1 being the original timeseries and scale $\mathrm{n}$ being the timeseries downsampled $\mathrm{n}$ times). This allows a characterisation of the complexity that exists across different frequency ranges within a single timeseries. Multiscale entropy was calculated using the MSE tool, as implemented in C (http://physionet.org/physiotools/mse). In both the SampEn and MSE calculations, a template length, $\mathrm{m}$, of 2 and a matching threshold, $r$, of $20 \%$ of the standard deviation of the original timecourse was used, as in previous studies involving biological signals (Bruce et al., 2009, Hauge et al., 2011). Timeseries were normalised to have a mean of 0 and variance of 1 prior to entropy calculation.

\subsubsection{Participants}

Data from two separate groups of participants were used: Group 1 underwent EEG only and consisted of twenty-four participants ( 12 female; mean age $=22.2$ years, age range $=$ 18 - 38 years); Group 2 underwent fMRI and MRS and consisted of twenty-eight participants (10 female; mean age $=22.3$ years, age range $=18-32$ years). Eight participants from group 2 were excluded due to them not following the task, along with four who had excessive head movement, leaving 16 for the analysis ( 6 female; mean age $=23.6$ years, age range $=19-32$ years). Both groups were healthy volunteers recruited via advertisements at universities in Montreal and Ottawa. All participants were right-handed, with no history of neurological or psychiatric disorders. Participants were screened for regular recreational drug use, including heavy alcohol usage. All participants gave their written informed con-

sent and were paid for their participation. Approval for the study was obtained from the ethics committees at McGill University, the Université de Montréal, and the Institute of Mental Health Research. 


\subsubsection{EEG acquisition and analysis}

Participants performed counterbalanced four minute resting eyes-closed (EC) and eyesopen (EO) periods. Participants were instructed to relax and allow their mind to disengage during these periods.

EEG were recorded using 30 silver-silver chloride cup electrodes attached to an electrocap (Quik-Cap; NeuroScan), with clectrodes placed at Fp1, Fp2, F7, F3, Fz, F4, F8, FT7, FC3, $\mathrm{FCz}, \mathrm{FC} 4, \mathrm{FT} 8, \mathrm{~T} 7, \mathrm{C} 3, \mathrm{Cz}, \mathrm{C} 4, \mathrm{~T} 8, \mathrm{TP} 7, \mathrm{CP} 3, \mathrm{CPz}, \mathrm{CP} 4, \mathrm{TP} 8, \mathrm{P} 7, \mathrm{P} 3, \mathrm{Pz}, \mathrm{P} 4, \mathrm{P} 8$, $\mathrm{O} 1, \mathrm{Oz}$, and $\mathrm{O} 2$ according to the extended International 10-20 Systems. The reference electrode was positioned on the tip of the nose. Blink and eye movements were monitored with electrodes above and below the left eye (vertical electrooculogram, VEOG) and at the right and left outer canthi of the eyes (horizontal electrooculogram, HEOG). The electrode impedance was maintained as less than $5 \mathrm{k} \Omega$. The EEG and EOG signals were amplified using a low-pass filter of $100 \mathrm{~Hz}$ and a notch filter of $60 \mathrm{~Hz}$, and digitized at a $1000 \mathrm{~Hz}$ sampling rate using the EEG recorder (Neuroscan SynAmps RT amplifier; NeuroScan Inc,, Charlotte, NC, USA).

EEG data analysis was performed using EEGLAB toolbox (http://sccn. ucsd.edu/eeglab/) (Delorme and Makeig, 2004) running under MATLAB. Data were filtered using a low-pass filter of $60 \mathrm{~Hz}$ and a high-pass filter of $1 \mathrm{~Hz}$. Epochs with irregular noise were identified and rejected using a computer algorithm and by inferences from visual inspection (Delorme et al., 2007). Typical physiological artifacts (e.g., eye blinks, eye movement, and muscle potentials) were retained and an extended infomax independent component analysis (ICA) performed to obtain 32 ICs from EO and EC in each participant. For each IC, an equivalent current dipole was estimated (DIPFIT 2.2, EEGLAB plug-in using Fieldtrip toolbox functions; Robert Oostenveld). ICs representing the typical physiological artifacts and electrode artifacts were identified by visual inspection of their time course data, multi-trial ERP-image plots, the power spectrum, scalp topography, and dipole. On average, 12.38 ICs were rejected from each participant's data. The remaining ICs were back-projected onto the scalp electrodes to obtain artifact-free EEG data. Finally, the de-noised EO and EC data were split into $8 \times 30 \mathrm{~s}$ segments per subject.

Data were analysed after being resampled at two different frequencies - 60 and $500 \mathrm{~Hz}$. Sample entropy at a single timescale was calculated using the $60 \mathrm{~Hz}$ data to match the 
band-pass filter frequency range. Multiscale entropy was calculated using the $500 \mathrm{~Hz}$ data, as this higher frequency gives a large number of datapoints for the coarse-graining procedure to be carried out on, and thus a better frequency resolution than would be possible with the lower frequency data.

Entropy measures were calculated for each 30 second segment in both the EO and EC conditions for all electrodes and then averaged across segments to give one value per electrode per subject. EO and EC entropy values for each electrode were compared across participants (paired t-tests, two tailed). In a similar manner, multiscale entropy values were calculated with a maximum timescale of 40 . For ease of interpretation of the multi-dimensional results, values for electrodes combined into anterior (F3, FZ, F4, FC3, FCZ, FC4), mid (C3, CZ, $\mathrm{C} 4, \mathrm{CP} 3, \mathrm{CPZ}, \mathrm{CP} 4)$, and occipital (O1, OZ, O2, P3, PZ, P4) groups were then compared between the EO and EC conditions (paired Student's t-tests, two-tailed). The significance level for EEG analyses was $p<0.05$. FDR correction was used where relevant to account for multiple comparisons.

\subsubsection{MRI}

MRI scanning was carried out at two different centres (fMRI - Montreal Neurological Institute, McGill University; MRS - Unité de neuroimagerie fonctionelle, Université de Montréal). Siemens 3 Tesla Trio MRI scanners were used at both locations. The mean time between scans for the analysed participants was 3.6 days (range $=1-10$ days).

\section{MRI - MRS acquisition and analysis}

Single voxel edited ${ }^{1} \mathrm{H}$ MR spectra were acquired using the MEGA-PRESS method (Marjanska et al., 2007, Mescher et al., 1998) with a body coil transmit and 12-channel receive headcoil. Using a high resolution $\mathrm{T}_{1}$-weighted anatomical image (MPRAGE; FoV $=205 \mathrm{x}$ $205 \mathrm{~mm}^{2}$; spatial resolution $=1 \times 1 \times 1 \mathrm{~mm}^{3} ; \mathrm{T}_{\mathrm{E}}=3.02 \mathrm{~ms} ; \mathrm{T}_{\mathrm{R}}=2000 \mathrm{~ms}$; flip angle $=$ $5^{\circ}$ ), volumes of interest (VOI) were located in the $\mathrm{mPFC}$ and the left insula (see Figure 2.3 for locations). In order to achieve consistent VOI positioning, placement was done by the same investigator for all participants according to easily identifiable anatomical landmarks: mPFC VOIs $\left(48 \times 21 \times 21 \mathrm{~mm}^{3}\right)$ were placed anterior to the genu of the corpus callosum, 
parallel to the AC-PC plane; left insula VOIs $\left(23 \times 48 \times 27 \mathrm{~mm}^{3}\right)$ were aligned with the line of the insula cortex in an anterior-posterior direction with the most anterior edge of the VOI aligned to the anterior limit of the insula.

Difference spectra were analysed using LCModel 6.2-1A (Provencher, 1993, 2001) using the basis set which included an experimentally measured metabolite-nulled macromolecular spectrum (average from 10 participants) and the experimentally measured spectra from 100 $\mathrm{mM}$ phantoms of $\mathrm{N}$-acetylaspartate (NAA), gamma-amino butyric acid (GABA), glutamate (Glu), and glutamine (Gln) with $\mathrm{pH}$ adjusted to 7.2 and at $37^{\circ} \mathrm{C}$. The LCModel fitting was performed over the spectral range from 0.5 to $4.0 \mathrm{ppm}$. No baseline correction, zerofilling, or apodization functions were applied to the in vivo data prior to LCModel analysis. Only results with the Cramér-Rao lower bounds below $20 \%$ were included in the analysis. Concentrations with CRLB $>20 \%$ were classified as not detected.

Metabolite of interest concentrations as a ratio to NAA were used in all subsequent steps. Of the 16 participants with usable fMRI data, 13 had usable glutamate+glutamine (Glx) results. Unfortunately, too few participants had usable GABA data for each region to allow correlation with this metabolite $(\operatorname{mPFC} n=6$, insula $n=9)$.

\section{MRI - FMRI acquisition and analysis}

Functional EPI scans were acquired using a body coil transmit and 32-channel receive headcoil. Forty-seven slices aligned at $-30^{\circ}$ from the AC-PC plane and covering the whole brain were acquired per volume, with a total of 467 volumes being acquired (1060 s) over the task run $\left(\mathrm{FoV}=205 \times 205 \mathrm{~mm}^{2}\right.$; spatial resolution $=3.2 \times 3.2 \times 3.2 \mathrm{~mm}^{3} ; \mathrm{T}_{\mathrm{E}}=25 \mathrm{~ms}$; $\mathrm{T}_{\mathrm{R}}=2270 \mathrm{~ms}$; flip angle $=90^{\circ}$ ). The first five volumes were discarded. High-resolution $\mathrm{T}_{1^{-}}$weighted anatomical image were also acquired (MPRAGE; FoV $=205 \times 205 \mathrm{~mm}^{2}$; spatial resolution $=1 \times 1 \times 1 \mathrm{~mm}^{3} ; \mathrm{T}_{\mathrm{E}}=3.02 \mathrm{~ms} ; \mathrm{T}_{\mathrm{R}}=2000 \mathrm{~ms} ;$ flip angle $=5^{\circ}$ ).

The session consisted of two long eyes-open (EO) and two long eyes-closed (EC) periods (2 x $120 \mathrm{~s}, 212$ volumes, alternating and counterbalanced across participants), that followed on from a sequence of short EO and EC blocks (255 volumes), the data for which were not analysed here. EC conditions were indicated by a single short tone and EO by a short double tone (100 ms and $2 \times 100 \mathrm{~ms}$, respectively). Small EO and EC icons were also displayed to 
inform participants of the current condition in case of confusion. Participants were observed using a simple camera setup to ensure that they followed the task instructions.

Preprocessing of the fMRI data was done using the FSL suite of tools (Smith et al., 2004, Woolrich et al., 2009). Functional images were, realigned, slice-time corrected, brainextracted, and high-pass filtered (100 s). Data not to be analysed (short EO and EC task) were then discarded. Each subject's data was entered into an ICA (MELODIC) to identify noise components resulting from head-movement, breathing, heart-rate, etc (Kelly et al., 2010). These noise component timecourses, along with the six head-motion parameters, were then regressed out of the functional data. Finally, data were split into EO and EC segments. There was no difference in mean absolute head displacement (Van Dijk et al., 2011 ) between the EO and EC condition $(\mathrm{t}=0.46, \mathrm{p}=0.65)$, nor in heart-rate peak-topeak variability $(\mathrm{t}=-0.06, \mathrm{p}=0.95)$, as estimated from the timeseries within the PESTICA cardiac map (http://www.nitrc.org/projects/pestica/), thresholded to include only the arteries.

The grey-matter within the MPFC and insula MRS voxels were taken as regions of interest (ROIs). To create the grey matter ROIs for each subject, segmented grey-matter maps were thresholded at 0.95 to produce binary images that were then masked with the mPFC MRS region.

Sample entropy was calculated in fMRI at a single timescale only, as a relatively small number of data-points were available per block, meaning that reliable entropy estimation would not be possible with any degree of downsampling. As a comparison with the entropy measure, the SD of each signal (EO \& EC, mPFC and insula) was also calculated as an alternative, linear (as compared to the logarithmic entropy measure), measure of signal complexity.

In a first step, entropy and SD values were calculated for each grey-matter voxel across the whole brain for EO and EC. Grey-matter masks were produced for each subject from their segmented anatomical maps, binarised with a threshold of 0.51 (in a range of 0 to 1). The timecourse from each voxel for each $\mathrm{EO}$ and $\mathrm{EC}$ block was then used to calculate the entropy and SD values. Voxel entropy and SD values were normalised within subjects by subtracting the whole brain grey-matter mean and dividing by the standard deviation (i.c., using the standardised z-score). The z-maps for each measure were then averaged 
for each condition and smoothed with an $8 \mathrm{~mm}$ FWHAM Gaussian kernel. Finally, EO and EC conditions were compared using a whole-brain paired-sample t-test (cluster significance level pFWE $=0.05, \mathrm{z}$-threshold $=2.3$ ). The same procedure was used to calculate entropy and SD values for the grey matter within the mPFC and insula MRS voxels for the EO and EC conditions. Mean entropy and SD values were then calculated for each condition and compared for each ROI (paired t-tests, two-tailed).

In a second step, partial correlations (Spearman) were calculated between $\mathrm{MPFC}$ and insula entropy and SD values, and MRS measures from the same regions, with subject age and the density of grey-matter within the MRS regions included as control variables. The correlations between regional entropy and the alternate region's MRS values were also calculated. Correlations were firstly calculated using the combined glutamate+glutamine concentrations (in relation to NAA), followed by correlations with each metabolite separately.

Finally, to investigate the likelihood of obtaining from a set of random signals (with the same mean and standard deviation) the same mean entropy values as that which were measured here within the $\mathrm{mPFC}, \mathrm{mPFC}$ and insula ROI timeseries were randomly permuted ( 5000 permutations) and the entropy value calculated each time to produce a distribution against which the experimental value was compared (Student's t-test, two-tailed). In a similar way, the correlation between entropy and glutamate was tested against the entropy values generated by the random permutations to establish the likelihood of obtaining the same correlation by chance.

\subsection{Results}

\subsubsection{EEG - entropy EO \& EC}

Averaging entropy across all electrodes, single scale entropy was higher in the EC condition than in $\mathrm{EO}(\mathrm{EC}=1.93 \pm 0.11, \mathrm{EO}=1.86 \pm 0.11, \mathrm{t}=4.56, p<0.001)$. When each electrode was taken individually, the same effect was seen at all electrodes other than FC4, F4, T7, and FT7 $\left(p_{\mathrm{FDR}}<0.05 ;\right.$ Figure $\left.1 \mathrm{~A}\right)$.

Multiscale entropy was higher in the EC than EO condition for each of the electrode groups 
at somewhat different temporal scales (Figure 1B), although all beginning at a scale of 11 or 12 (anterior $=$ scale $11-24 ; \mathrm{mid}=$ scale $12-19 ;$ occipital $=$ scale $12-20$ ). In the case of the occipital group, EO entropy becomes larger than EC at a temporal scale of 33 , continuing until a scale of 40 (excluding 34 ).

\subsection{2 fMRI - entropy EO \& EC}

Comparing entropy values across the whole brain, at a significance level of $p_{\text {FWE }}<0.05$ entropy was found to be higher during EO than EC in the lingual gyrus, intracalcarine cortex, and precuneus; at a lower, uncorrected, threshold of $p_{\text {unc }}<0.005$ (cluster extent $(\mathrm{k})$ $>50$ voxels), entropy was also seen to be higher in the regions including the left precentral gyrus and right temporal pole (Figure 5.2, Table 5.1A). No regions were found to have higher entropy in EC compared to EO at the $p_{\text {FWE }}<0.05$ threshold; however, at the lower threshold clusters of higher entropy were seen in the right dorsal anterior cingulate cortex (dACC) and bilateral lateral prefrontal cortex (PFC) (Figure 5.2, Table 5.1A).

At a threshold of $p_{\mathrm{FWE}}<0.05$, no regions displayed altered SD between the conditions. However, at the lower threshold of $p_{\text {unc }}<0.005$ ( $\mathrm{k}>50$ ), multiple regions differed between

the two conditions. In the eyes-open condition, SD was higher in regions such as the bilateral lateral occipital cortex and right inferior frontal gyrus (Figure 5.2, Table 5.1B). In the eyes-closed condition, SD was higher in bilateral auditory cortex, precuneus and PCC, amongst other regions (Figure 5.2, Table 5.1B).

Within the mPFC ROI, entropy was seen to be higher during the EC condition when compared to EO ( $t=2.27, p=0.038$; Figure 5.3). Similarly, in the left insula entropy was marginally higher during $\mathrm{EC}(\mathrm{t}=2.16, p=0.047)$. There was no difference in standard deviation in either region between $\mathrm{EO}$ and $\mathrm{EC}$ ( $\mathrm{mPFC} \mathrm{t}=-0.87, p=0.39$; insula $\mathrm{t}=-1.37$, $p=0.19)$.

Entropy values within the mPFC and insula were significantly different from values obtained from random permutations in all subjects for both the EO and EC conditions $(p<0.001)$. Correlation coefficients from the comparison of random mPFC EC entropy values and mPFC Glu/NAA were significantly lower than the experimental value $(p<0.001)$. 
A)
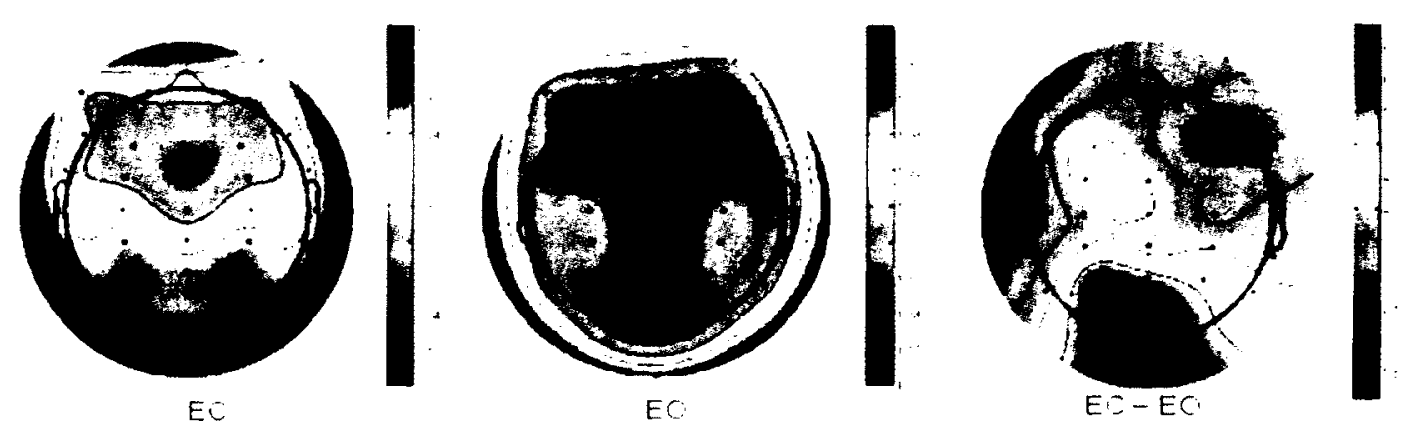

B)

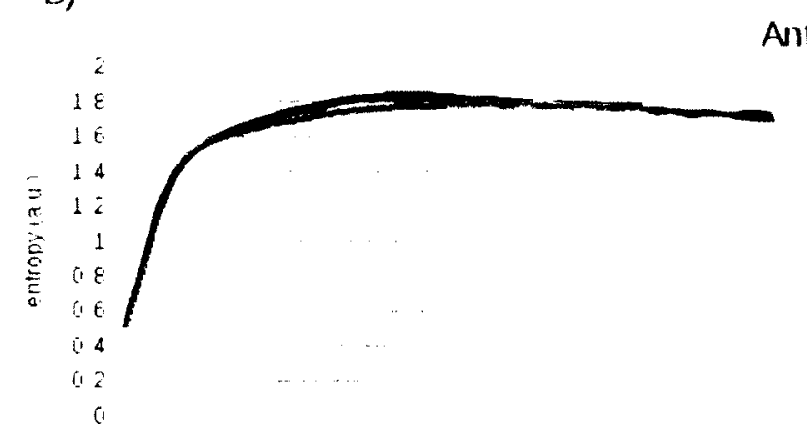

Anterior

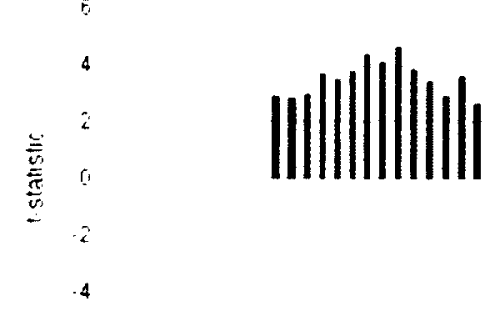

Middle
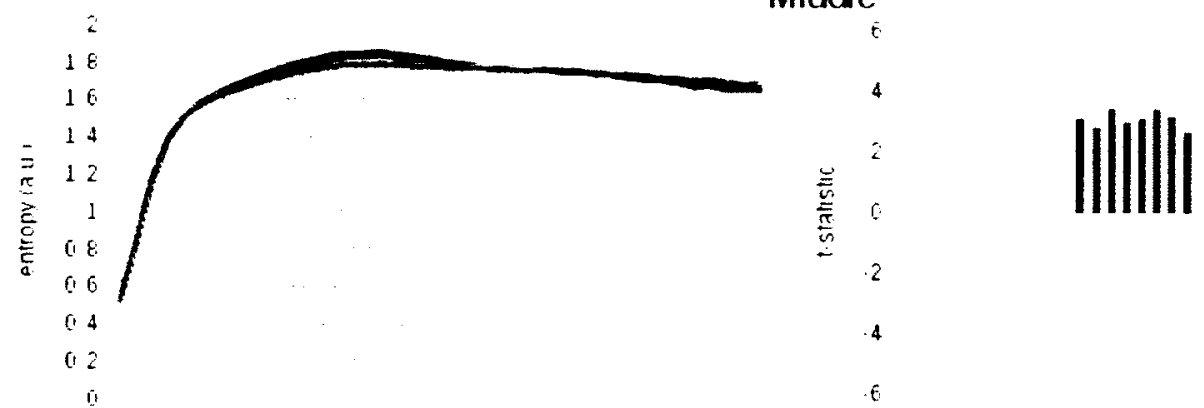

EC

Posterior
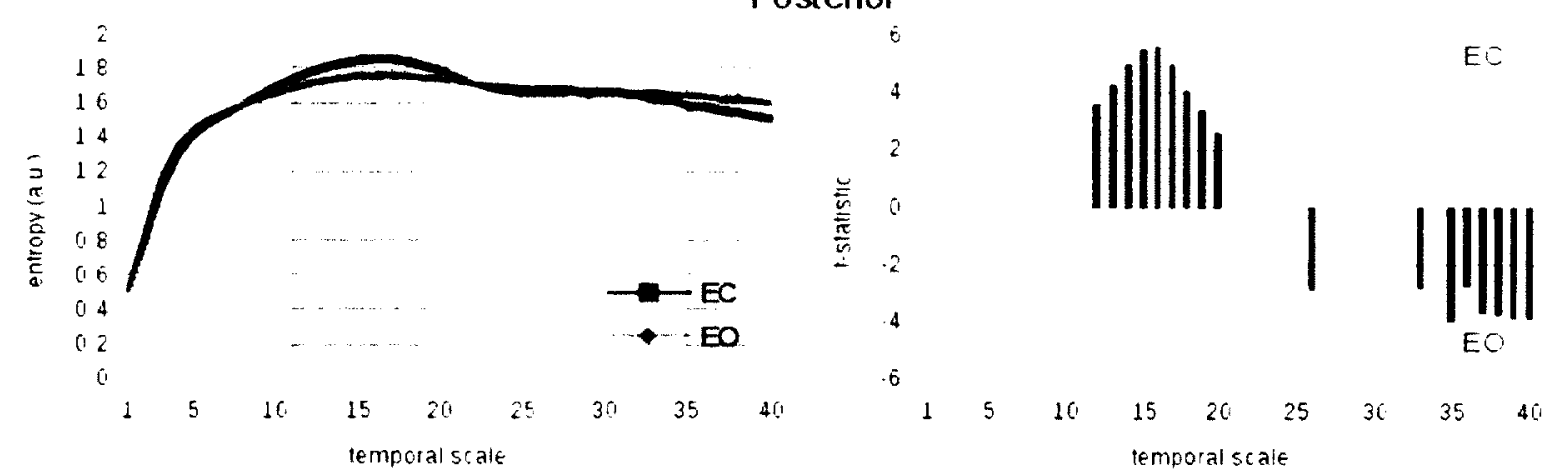

Figure 5.1: A) Single temporal scale entropy values for all clectrodes in eyes-closed and eyes-open conditions, along with t-statistics from paired-sample t-test between conditions. The largest difference between EC and EO can be seen in occipital and anterior midline regions. (B) Multiscale entropy comparison between eyesclosed and eyes-open for anterior, middle, and posterior electrode groupings (see Methods for definitions). Entropy can be seen to be higher during the EC than EO condition, other than in posterior regions at high temporal scales where EO shows higher entropy. 


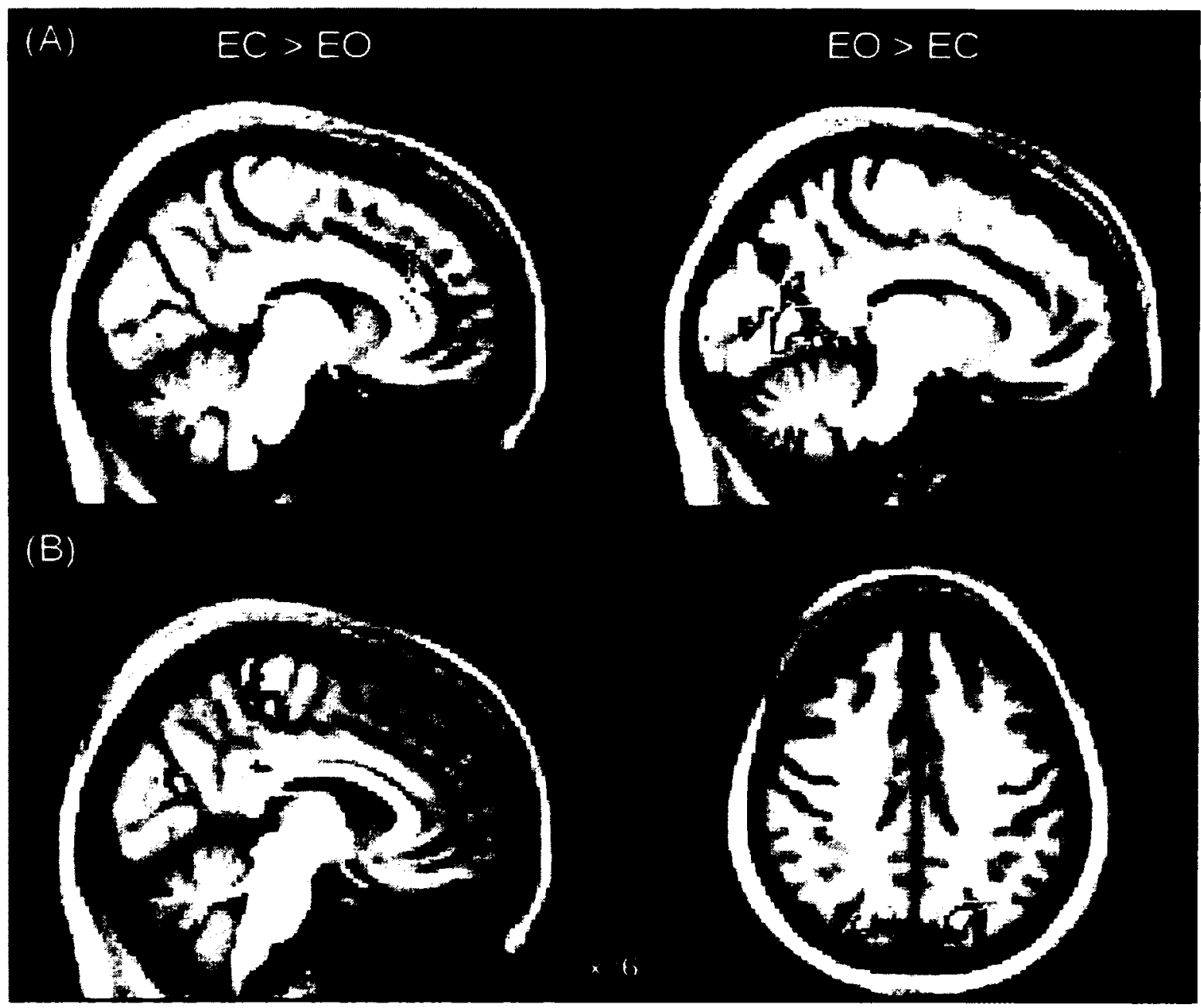

Figure 5.2: (A) Contrast between eyes-closed and eyes-open entropy (right), showing increased entropy in the ACC during eyes-closed, and the converse contrast (left) showing increased entropy in the precuneus and occipital cortex during eyesopen. (EC EO $p_{\text {unc }} 0.005 . \mathrm{k} .50$; EO EC $p_{\text {FWE }} 0.05 . z \cdots 2.3$ ).

Contrast between eyes-closed and eyes-open SD (right). showing greater SD during eyes-closed in the $\mathrm{PCC}$ precuneus and greater SD in the lateral occipital cortex during eyes-open $\left(p_{\text {une }} \quad 0.005, \mathrm{k}, 50\right)$. Results are displayed on group mean anatomical image in MNI space. 


\begin{tabular}{|c|c|c|c|c|c|}
\hline Region & K & Z-score & $\mathrm{x}$ & $y$ & $\mathrm{z}$ \\
\hline \multicolumn{6}{|l|}{ (A) entropy EC > EO } \\
\hline L IFG & 186 & 3.48 & -52 & 20 & 6 \\
\hline \multirow[t]{2}{*}{ R LPFC } & 175 & 3.76 & 28 & 40 & 12 \\
\hline & 53 & 3.12 & 58 & 38 & 0 \\
\hline R ACC & 123 & 3.2 & 10 & 34 & 22 \\
\hline \multicolumn{6}{|l|}{ entropy $\mathrm{EO}>\mathrm{EC}$} \\
\hline $\mathrm{L} / \mathrm{R}$ ICC/lingual & 860 & 4.57 & -2 & -54 & 6 \\
\hline \multirow[t]{2}{*}{ gyrus/precuneus } & 138 & 3.39 & -10 & -54 & 60 \\
\hline & 112 & 3.3 & 0 & -40 & 66 \\
\hline L PSG & 90 & 3.35 & -58 & -18 & 44 \\
\hline cerebellum & 85 & 3.24 & -8 & -64 & -38 \\
\hline $\mathrm{R}$ temporal pole & 74 & 4.03 & 36 & 6 & -32 \\
\hline R operculum & 50 & 3.53 & 50 & -10 & 24 \\
\hline \multicolumn{6}{|l|}{ (B) $\mathrm{SDEC}>\mathrm{EO}$} \\
\hline $\mathrm{L}$ auditory cortex & 1298 & 4.58 & -34 & -18 & 30 \\
\hline $\mathrm{R}$ precuneus & 729 & 3.78 & 12 & -42 & 56 \\
\hline L lingual gyrus & 474 & 3.55 & -20 & -48 & 4 \\
\hline $\mathrm{R}$ auditory cortex & 200 & 3.93 & 34 & -24 & 16 \\
\hline $\mathrm{R}$ cuncus & 171 & 4.02 & 8 & -68 & 18 \\
\hline \multirow[t]{2}{*}{ PCC } & 120 & 3.35 & -2 & -34 & 28 \\
\hline & 107 & 3.41 & -12 & -26 & 36 \\
\hline \multicolumn{6}{|l|}{$\mathrm{SD} \mathrm{EO}>\mathrm{EC}$} \\
\hline $\mathrm{R}$ IFG & 383 & 4.1 & 56 & 30 & 24 \\
\hline \multirow[t]{2}{*}{ R lat. occ cortex } & 252 & 3.6 & 18 & -72 & 48 \\
\hline & 77 & 3.72 & 40 & -80 & 16 \\
\hline L lat. occ. cortex & 200 & 3.67 & -16 & -76 & 42 \\
\hline $\mathrm{L}$ temporal pole & 82 & 3.76 & -36 & 10 & -28 \\
\hline
\end{tabular}

Table 5.1: Regions showing differences in (A) entropy, and (B) standard deviation (SD) between the EO and EC condition. Coordinates are in MNI standard space. $\mathrm{IFG}=$ inferior frontal gyrus, $\mathrm{LPFC}=$ lateral prefrontal cortex, $\mathrm{ACC}=$ anterior cingulate cortex, $\mathrm{ICC}=$ intra-calcarine cortex, $\mathrm{PSG}=$ precentral gyrus, $\mathrm{PCC}=$ posterior cingulate cortex, lat. occ. = lateral occipital. 


\subsubsection{MRI - entropy and biochemistry}

The correlation between entropy in the mPFC during EC and Glx/NAA had a trend towards significance $(\mathrm{R}=-0.51, p=0.073)$. Entropy in the $\mathrm{mPFC}$ during $\mathrm{EC}$ was found to correlate with Glu/NAA concentrations in the same region $(\mathrm{R}=-0.76, p=0.0068$; Figure 4), but not with $\mathrm{Gln} / \mathrm{NAA}(\mathrm{R}=0.11, p=0.72)$. During the EO condition, no correlation was found between mPFC entropy and any of mPFC Glx/NAA $(\mathrm{R}=-0.39, p=0.19)$, Glu/NAA (R $=-0.41, p=0.16$; Figure 5.3) or $\mathrm{G} \ln (\mathrm{R}=0.02, p=0.96)$. No correlation was observed between $\mathrm{mPFC}$ entropy during either $\mathrm{EO}$ or $\mathrm{EC}$ and the concentration of Glu/NAA or Gln/NAA in the left insula. Similarly, no correlation was found between metabolites and mPFC SD during either condition.

In the left insula, no correlation was found between entropy and insula metabolite levels during either condition. Similarly, no correlation was seen between signal standard deviation and metabolite levels. During the EC condition, entropy within the insula was found to correlate with Glx/NAA, but not with either metabolite separately, suggesting that the Glx/NAA correlation may be spurious.

\subsection{Discussion}

Pattens of entropy changes between EO and EC rest were broadly comparable in the context of both EEG and fMRI. Single timescale entropy in EEG was higher over the majority of electrodes in EC than EO. This difference was mirrored in the multiscale entropy results at low-to-medium timescales (corresponding to high-to-medium frequencies), switching to greater entropy in the $\mathrm{EO}$ condition in the posterior electrodes at lower timescales. EC entropy remains higher than EO at lower timescales in the anterior electrodes than it does in the middle and posterior ones. The fMRI results thus fit with the multiscale entropy results at medium-to-low timescales in that EC entropy is higher than EO in the anterior regions, whilst posterior regions show higher entropy in the EO condition.

More specifically, the AMR results localise the differences in entropy to the $\mathrm{dACC}$ and lateral PFC (EC $>$ EO), and the intracalcarine cortex/lingual gyrus $(E O>E C)$. The entropy increase in the visual areas during eyes-open would seem to fit well with the increased 


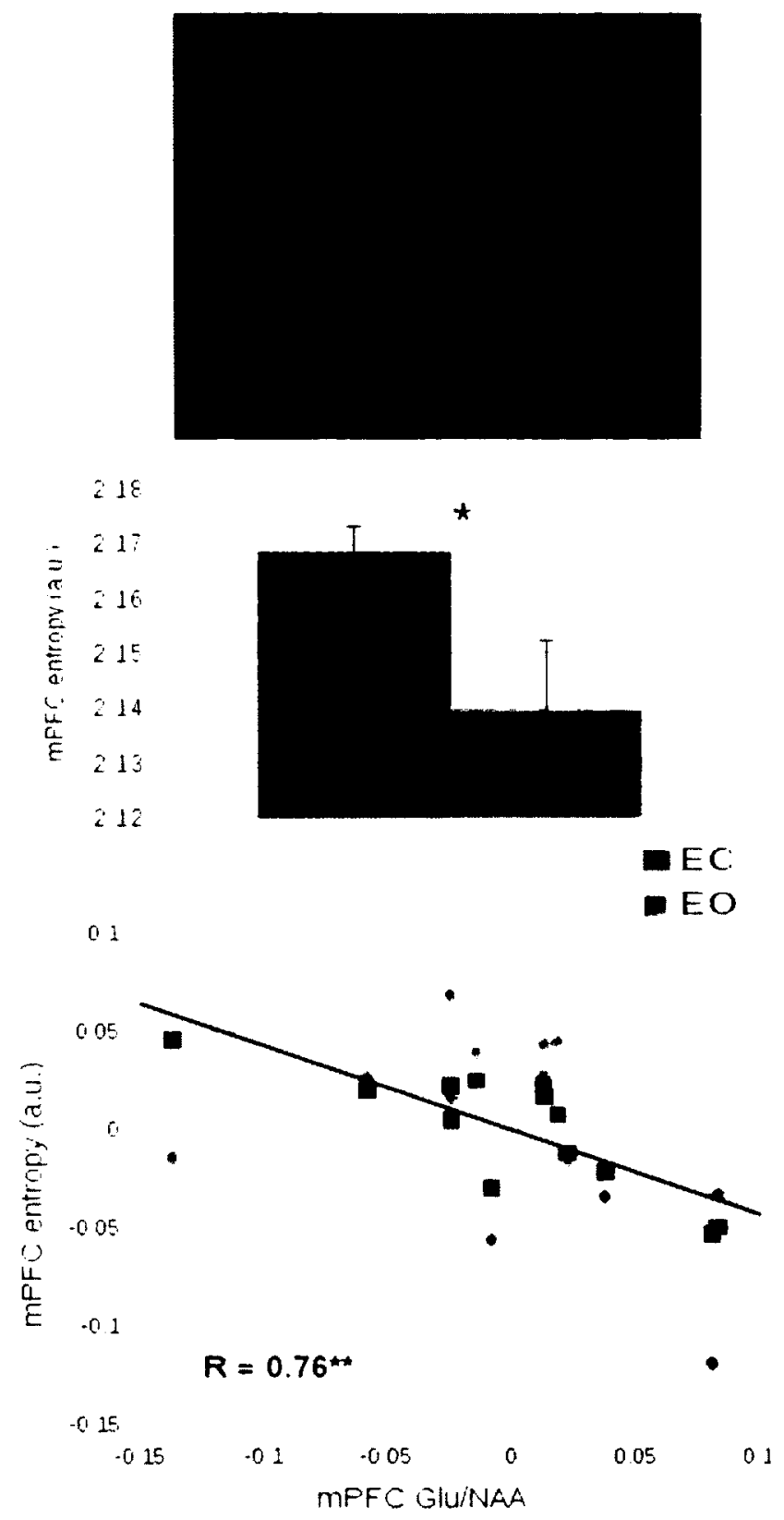

Figure 5.3: Entropy results from mPFC ROI (shown in red on group mean anatomical image). $\mathrm{mPFC}$ is higher during the eves-closed than eyes-open condition ( $p$ 0.05). Eyes-closed entropy values correlate with mPFC Glu NAA concentrations $(p \cdot 0.01)$. 
sensory input to this region in this condition. Following a similar line, the increase in entropy in the dACC and lateral PFC during EC may be linked to the proposed role that these regions play in uncertain contextualisation (Reynolds et al., 2012, Roy et al., 2012). The EC condition may represent a more 'uncertain' context than the EO condition (in which the participant has external cues for reference), and as such activity complexity is increased in line with the greater regional involvement. The conclusion that entropy increases are linked to 'task-related' activity is supported by previous studies that have found just such region-specific increases in entropy during particular tasks (Misić et al., 2010).

Variance fMRI results differed from those obtained using entropy. During the EC condition, SD was higher in, amongst other regions, the PCC and precuneus. Higher SD in these regions may be linked to the role that these regions play in the so-called default mode network (DMN), potentially reflecting increased inwardly directed thoughts or mindwandering (Christoff, 2012) that is suppressed during the eyes-open condition. During this latter condition, SD was increased in regions that have been described as being components of the visual and attention networks (Smith et al., 2009), both of which would be understandable in the context of increased visual input and a switching of attention to the external environment.

It is thus notable that both SD and entropy changes appear to be context-relevant but that the regions highlighted differ between the two measures. An explanation for this may lie in the linear versus nonlinear nature of each identifying differing activity characteristics across the distinct regions. Support for this position comes from recent work by Gultepe and He (Gultepe and He, 2012) that identified a greater non-linearity to the resting-state activity in, for example, the visual cortex, with the greatest linearity of activity within the DMN. This pattern fits well with the current results, where nonlinear entropy changes are seen in the visual cortex whilst linear SD changes are seen in the PCC (a component of the DMN).

Within the mPFC ROI, entropy was found to be higher during eyes-closed than eyes-open, with no difference in SD. The functional source of these FMRI entropy values is supported by previous work that has linked increased PFC entropy to increased oxygen turnover using near-infrared spectroscopy (Jausovec and Jausovec, 2010). In addition, eyes-closed entropy correlated negatively with mPFC Glu/NAA concentrations (specific to Glu/NAA and to the $\mathrm{MPFC}$, as compared to insula), meaning that with increasing glutamate within the 
region there was a reduction in entropy. Although no significant correlation was found with eyes-open entropy, the scatter plot (Figure 5.3) shows that this is likely to be due to a larger degree of variability in entropy values across subjects (Levene's test, $\mathrm{p}=0.014$ ) within a similar relationship trend. No correlation was found between SD values and transmitter concentrations (although the unfortunate lack of GABA values leaves open the intriguing possibility that a correlation may have been found between SD and that transmitter given the inhibition/excitation balance important in neural activity (Lauritzen et al., 2012). The link between entropy and glutamate may represent a link between simulation studies that incorporate realistic excitation/inhibition properties to model brain dynamics (Deco and Jirsa, 2012), although this suggestion is very preliminary.

Finally, a number of limitations of the present study must be considered. The use of single timescale entropy analysis in fMRI was necessary with the data available; it would be desirable, however, to apply multiscale entropy to this data type, for which longer resting periods would seem necessary. The unfortunate lack of GABA MRS results means that the potential links between activity dynamics and this transmitter remain to be investigated. Similarly, the fact that the EEG and fMRI datasets came from different participant groups meant that EEG dynamics and biochemistry could not be compared. Future studies where both EEG and MRS are carried out in the same people are thus necessary. Finally, the final number of fMRI participants that could be used was somewhat low, meaning that repetition of the current results in a larger group is desirable.

To conclude, ongoing activity dynamics in the eyes-closed resting-state differed from those in the eyes-open resting-state. This was shown in both EEG and fMRI using entropy and $\mathrm{SD}$ as metrics. The results gained with the two techniques were found to be comparable and complementary, with the EEG results giving greater temporal detail to the differences and the fMRI allowing accurate regional localisation. Entropy and SD results were localised to different brain regions in a manner that suggests that they quantify different dynamics - entropy capturing nonlinear changes whilst SD captures linear. Finally, entropy values in the $\mathrm{mPFC}$ were found to correlate with glutamate concentrations in the same region, providing the first human in vivo link between activity dynamics and biochemistry. 


\subsection{Acknowledgements}

Many thanks to A. Perna and K. Dedovic for their help with subject recruitment, Prof. A. Longtin for his valuable input, Edward J. Auerbach, Ph.D. (Centre for Magnetic Resonance Research, University of Minnesota) for implementing MEGA-PRESS sequence on Siemens, the staff at the UNF and MNI for their skillful assistance, and Romain Valabregue, Ph.D. (Centre de NeuroImagerie de Recherche, Paris, France) for developing processing tools. The work was supported by grants to G.N. from the Canadian Institutes of Health Research and the Michacl Smith Foundation. 


\section{Chapter 6}

\section{Considerations for ${ }^{18} \mathrm{~F}$-flumazenil PET imaging of $\mathrm{GABA}_{\mathrm{A}}$ receptors}

\subsection{PET imaging}

Positron emission tomography - PET - exploits gamma rays to produce images of body structures and processes. Dating back to the 1950s, the procedure involves the injection of a positron-emitting nucleotide that has been introduced into a substance that is either used by the body or binds to some receptor site within the body. The exact nature of the drug used is determined by the structure or process that is of interest. After injection, the drug circulates with the blood to the target region, where it accumulates. As positrons (anti-electrons) are emitted by the tracer, they travel through the bodily tissues, eventually interacting with an electron and annihilating with the release of gamma waves in opposing directions. These waves are then detected by the PET camera, which generally takes the form of a ring around the body of the subject. Since the two waves were travelling in opposite directions, the point in space from which they originated can be calculated from the positioning and timing of the two detection points (Bailey et al., 2003). The totality of all the positions calculated can then be used to produce the PET image.

As an example, a commonly used tracer is radioactive fluorine-18 $\left({ }^{18} \mathrm{~F}\right)$. This has a half-life of approximately 110 minutes and can be inserted into a wide range of bio-active substances, including fluorodeoxyglucose (FDG). As FDG is an analogue of glucose, which the body takes up for energy production, ${ }^{18}$ F-FDG is ideal for imaging regions of the body that have high energy requirements, such as the brain and malignant tumours, as the tracer will accumulate there (as it cannot be used in energy metabolism) (Gallagher et al., 1978). Additionally, differences in accumulation levels give information regarding the amount of 
energy that is being used within a particular region. This information can then be used to infer, for example, differences in tumour development (Vallabhajosula, 2007) or neural activity (Phelps, 1981).

\subsection{The $\mathrm{GABA}_{\mathrm{A}}$ receptor and flumazenil}

As well as brain energy consumption, radiotracers can be inserted into compounds that bind to neural receptors. PET imaging can then be used to provide information about a particular receptor's distribution or functioning (Heiss and Herholz, 2006). In the context of the current work, the $\mathrm{GABA}_{\mathrm{A}}$ receptor is of interest. This receptor, for the neurotransmitter $\gamma$-aminobutyric acid (GABA), is distributed throughout the cortex and is the major inhibitory receptor in the brain (Olsen, 1981, Jacob et al., 2008). Composed of five subunits, the $\mathrm{GABA}_{\mathrm{A}}$ receptor has, in addition to the binding site for GABA itself, a modulatory benzodiazepine site. Occupation of this site increases the affinity of the receptor for GABA, this being the mode of action of the benzodiazepine class of indirect GABA agonists (Hanson and Czajkowski, 2008). In addition to the psychoactive benzodiazepine class of drugs, there is also a set of competitive benzodiazepine antagonists that will reversibly bind to the benzodiazepine site but produce little or no psychological effects in humans (Hunkeler et al., 1981), making them ideal for PET imaging (where one generally wishes to measure the brain in a 'natural' state without manipulating its activity pharmacologically). Most commonly used for this is flumazenil, radiolabelled with either ${ }^{11} \mathrm{C}$ or ${ }^{18} \mathrm{~F}$, the latter of these having the advantage of a longer half-life (la Fougère et al., 2011). Using this ligand, as is done in the current work, highly detailed maps of $\mathrm{GABA}_{\mathrm{A}}$ receptor density can be created.

Following injection, a radiolabelled ligand, such as flumazenil, is distributed in the body approximately according to a basic three-compartment model. This model consists of the free ligand in the blood plasma $\left(\mathrm{C}_{\mathrm{a}}\right)$, ligand that is non-specifically bound to tissue $\left(\mathrm{C}_{\mathbf{f}}\right)$, and ligand that is specifically bound to the tissue of interest $\left(\mathrm{C}_{\mathrm{b}}\right)$. With knowledge of the rate of transfer of the ligand across the blood brain barrier $\left(\mathrm{K}_{1}\right.$ and $\left.\mathrm{k}_{2}\right)$, and of the association/dissociation rate from the receptor $\left(\mathrm{k}_{3}\right.$ and $\mathrm{k}_{4}$ ), the change in concentrations across these compartments can be approximately calculated. However, acquiring the necessary

measurements to calculate the amount of ligand bound to the target receptor $\left(\mathrm{C}_{\mathrm{b}}\right)$ using 
this model requires the sampling of arterial blood (to obtain $\mathrm{C}_{\mathbf{a}}$ ), something that is impractical in most circumstances. As such, an approach that simplifies to a two-compartment model is often employed in human studies (Innis et al., 2007). This simplified reference tissue approach takes advantage of the very low doses of ligand used in comparison to receptor availability to assume a linear receptor ligand-associate rate, coupled with an assumption that non-specific binding is constant in the tissue being studied, to obtain an approximation of $\mathrm{C}_{\mathrm{b}}$ known as the binding potential (BP). In the current study the specific binding potential value used is the binding potential as compared to non-displaceable uptake - the $\mathrm{BP}_{\mathrm{ND}}$. This value can be calculated by comparing the concentration of of radioligand in the receptor-rich region of interest with a reference region that has no receptors once the system has reached an approximate equilibrium of transfer between the compartments (i.e, $\mathrm{k}_{3}$ and $\mathrm{k}_{4}$ reach a steady state) (Heiss and Herholz, 2006, Logan et al., 1996). In the case of flumazenil, equilibrium is reached after approximately 30 minutes, meaning that scans must be longer than this (usually 60 minutes). It should be noted that the $\mathrm{BP}_{\mathrm{ND}}$ does not represent a pure count of receptors but instead represents a function of receptor density and the affinity of the receptor for the ligand (Innis et al., 2007).

\subsection{Comparison of reference region location and volumes}

The simplified reference tissue method described is predicated on the reference region having no specific ligand binding. The pons was originally taken to fit this requirement for $\mathrm{GABA}_{\mathrm{A}}$ benzodiazepine sites, and so has been used in a number of FMZ-PET studies (Odano et al., 2009, Pearl et al., 2009). However, other studies have identified low but measurable benzodiazepine specific binding in this region (Frankle et al., 2012, Heiss and Herholz, 2006), leading to a switch to some studies using the cortical white matter as their reference tissue (Klumpers et al., 2008, la Fougère et al., 2011). This region itself has limitations, however, as it is not clear that the non-specific binding in white matter is directly comparable to that in cortical grey matter (Salmi et al., 2008). It is thus an open question as to which region is optimal for FMZ-PET binding potential estimation. In addition, as well as the particular

region to use, the optimal size of the reference region has not been much discussed in the literature, with the details of the region used in a particular study (i.e., the shape and size of the reference region) rarely reported. 
Given these questions, a brief comparison of reference region location and size was carried out. Spheres of various radii were located in the pons and cerebral white matter. Binding potentials were calculated for each and compared, along with the resulting signal-to-noise ratio. Results were then used to identify an optimal region location size and location for subsequent analyses.

FMZ-PET data was acquired using a Siemens ECAT-HRRT PET scanner. ${ }^{18} \mathrm{~F}$-flumazenil was synthesised as previously described (Massaweh et al., 2009). Head movement was minimised with a head-restraining adhesive band. A 6-minute transmission scan $\left({ }^{137} \mathrm{C}\right.$ point source) was first acquired for attenuation correction followed by an intravenous tracer injection (over $60 \mathrm{~s}$ ) of $260.7 \mathrm{MBq}\left( \pm 21.24 \mathrm{SD}\right.$ ) of ${ }^{18} \mathrm{~F}$-flumazenil. Subjects were instructed to close their eyes and remain awake. List-mode data was acquired for a period of 60 minutes and then binned into a series of 26 sequential sinograms of increasing duration, ranging from $30 \mathrm{~s}$ to $5 \mathrm{~min}$. PET data was reconstructed using a 3D OP-OSEM algorithm (10 iterations and 16 subsets) with correction for scatter, random coincidences, attenuation, decay, deadtime, and frame based motion correction (Costes et al., 2009, Hong et al., 2007, Hudson and Larkin, 1994). The resulting images had a resolution of $1.22 \times 1.22 \times 1.22 \mathrm{~mm}^{3}(256 \times$ $256 \times 207$ voxels).

In a first step, individual $\mathrm{T}_{1}$-weighted anatomical images (see proceeding chapter for MRI image acquisition details) were linearly aligned to the MNI template brain (all image-based analyses were carried out using the FSL suite of tools (Smith et al., 2004)). Anatomical images were then segmented into grey matter (GM), white matter (WM) and cerebrospinal fluid maps and the GM maps convolved with a $2.44 \mathrm{~mm}$ Gaussian kernel to simulate the resolution of the HRRT PET scanner (i.e., the minimum resolvable structure which equals $2 \times 1.22 \mathrm{~mm}$, the raw resolution of the scanner). Raw PET images were aligned to this 'simulated' PET image and the PET to anatomical and anatomical to MNI transforms combined and inverted.

Reference region centres were defined in MNI space on the standard MNI template. The central points were then converted into individual PET space through the previously calculated MNI to PET transformations. Spheres with diameters of incremental increases equal to the scanner resolution were then defined for each subject, starting at two times the minimum resolution and proceeding to the maximum radius that remained within the anatomical region $(2.5 \mathrm{~mm}, 3.75 \mathrm{~mm}, 5 \mathrm{~mm}, 6.25 \mathrm{~mm}$; see Figure 6.1 for sample refer- 


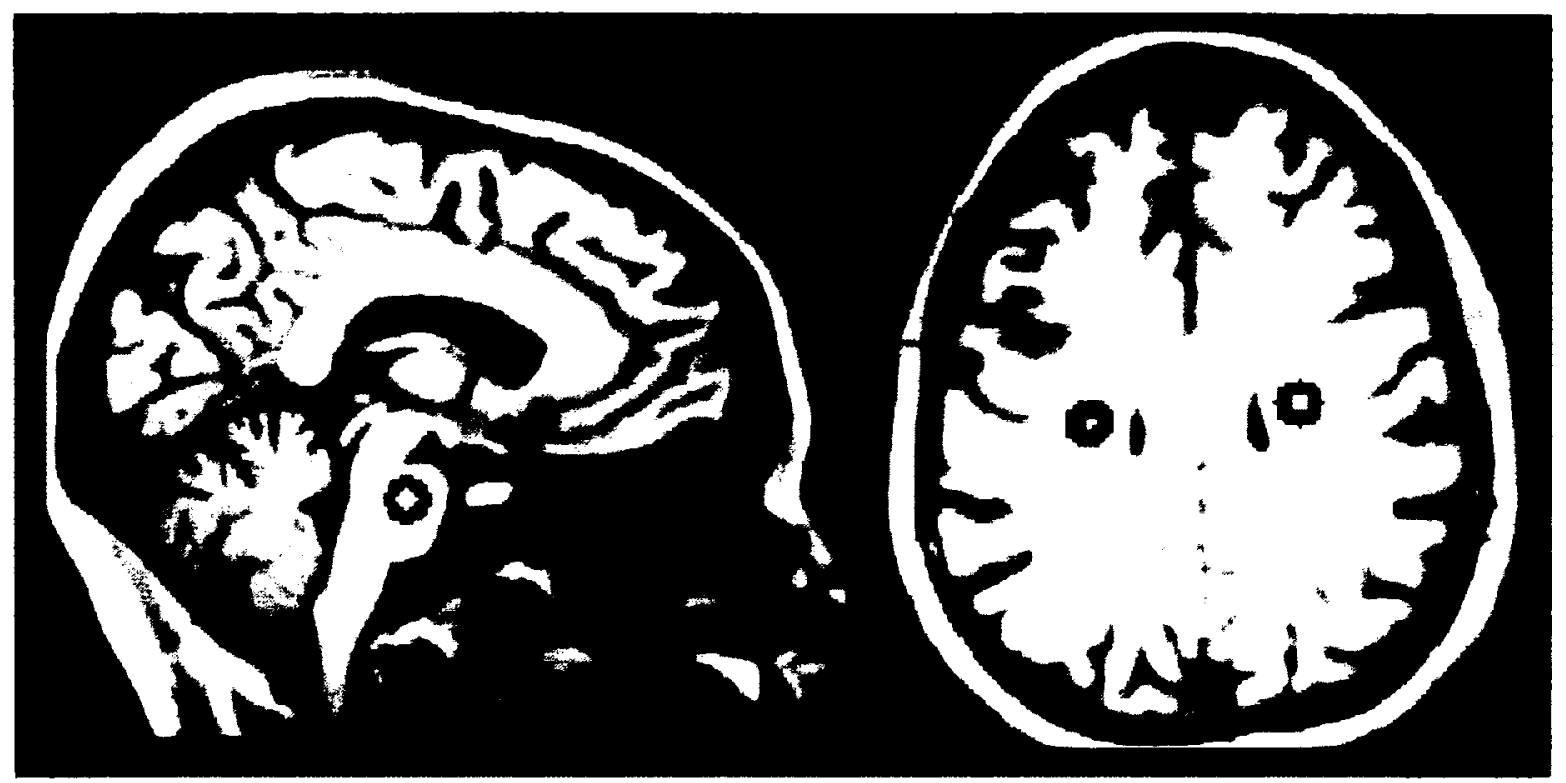

Figure 6.1: Reference region loration and sizes. Spheres are shown on a single subject's anatomical image.

ence regions). Whole brain $B P_{X D}$ majs were then (alculated for each reference region size using the standard Logan reference tissue graphical analysis method (Logan et al., 1996), implemented in MATLAB.

To compare reference regions. the BP.:D maps were firstly segmented into 48 bilateral anatomical ROIs, as defined in the Harvard-Oxford atlas (http://www . cma.mgh harvard. edu/fsl_atlas.html). ROI masks were aligned to individual PET space where they were used to extract the mean regional $B P_{x D}$ values and the standard deviation. BP $x \mathrm{D}$ values within each ROI were corrected for partial volume effects (PVC-BPND) using an inage based approach utilising the segmented anatomical MRI inages (Thiel et al., 2002). The signal-to-noise ratio (SNR), which represents the ratio of "true" signal to the level of noise in the acquired data. was then defined as the mean PVC-BP $\mathrm{ND}$ SD for each ROI (Hofheinz et al., 2011). Mean BP XD and SNR values for the whole brain were calculated for each subject by combining the values for the separate ROIs. Differences in whole-brain mean $B P_{X D}$ and SNR between reference regions and reference region sizes were tested via a two-way ANOTA and post-hoc Tukeys HSD tests. 
(a)

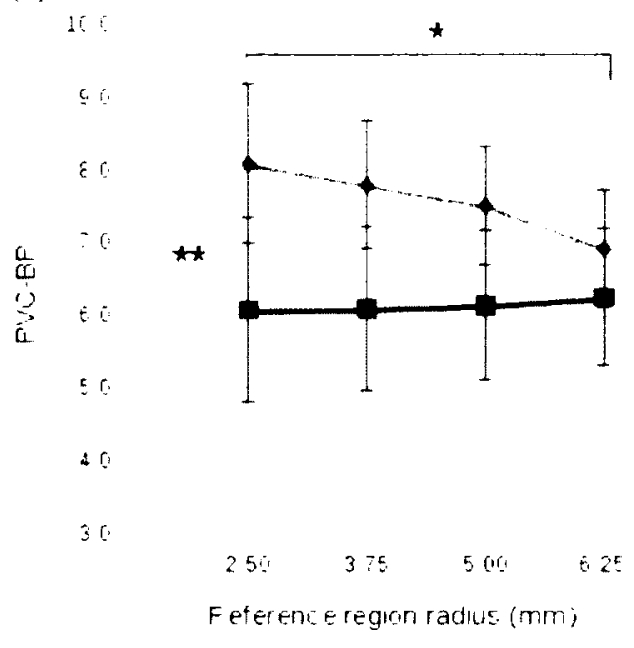

(b)

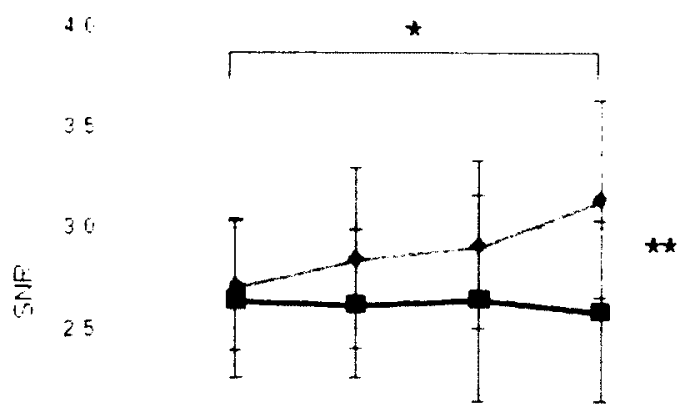

20

15

$250 \quad 375 \quad 506 \quad 625$

Fieference region radus (mm)

Figure 6.2: Comparison of (a) PVC-BPND and (b) SNR values across reference region location and sizes for the whole brain. The WM reference region gives a better SNR than the pons. ${ }^{*}$ denotes $p \cdot 0.01,{ }^{* *}$ denotes $p=0.001$.

To establish if the difference in $\mathrm{BP}_{\mathrm{ND}}$ values between reference regions reflects a change in the values obtained for each subject or instead represents a scaling of values that retains their distribution, a correlation between the values obtained for each ROI using each of the two reference regions was carried out (5 $\mathrm{mm}$ radius). All statistical analyses were carried out in MATLAB. The significance level was set at $\mathrm{p}$-0.05. FDR correction was used to rontrol for multiple comparisons.

Taking the cortex as a whole, an effect of both reference region $\left(\mathrm{F}_{1.192}-185, p \cdots 0.001\right)$ and reference region size on PYC-BP ND values was observed $\left(F_{3.192}=7.39, p=0.0085\right)$. Mean $\mathrm{PVC}-\mathrm{BP}_{\mathrm{ND}}$ values calculated using the pons reference region were lower than those calculated using WM (Figure 6.2a). Using the pons, mean PVC-BP ND values remain similar across all reference region sizes: in contrast, mean PVC-BP $\mathrm{ND}$ values were significantly. different between reference region sizes when the WM was used (see Figure 6.2a).

SNR ratio was higher with the $\mathrm{WM}$ reference region than the pons (Figure $2 \mathrm{~b} ; \mathrm{F}_{1,192}=$ $21.57, p=0.001$ ). With the WM. the SNR for the $6.25 \mathrm{~mm}$ region was significantly higher than that for the $2.5 \mathrm{~mm}$ region $\left(F_{3,192}=4.46, p=0.0056\right)$. No significant difference in 
SNR between region sizes was seen with the pons reference region.

Based on these results, $5 \mathrm{~mm}$ spheres in cortical W.M were chosen as the reference region for use in the subsequent analyses. WM was chosen rather than the pons given the slightly higher SNR that was obtained with this region. The $5 \mathrm{~mm}$ radius was selected instead

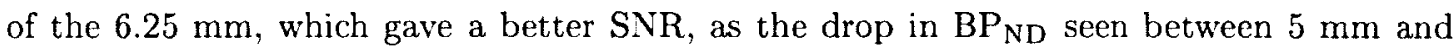
$6.25 \mathrm{~mm}$ towards the values obtained using the pons is suggestive of a seeping-in of specific benzodiazepine binding as the edge of the reference region gets closer to the cortical GM with the larger sphere (given that the pons has some specific binding (Frankle et al., 2012, Heiss and Herholz, 2006)). The $5 \mathrm{~mm}$ sphere thus appears to give 'cleaner' results.

\subsection{A marker of receptors or of neurons?}

The $\mathrm{GABA}_{\mathrm{A}}$ receptor is present on a large proportion of neurons throughout the cortex (Olsen, 1981). This ubiquity has led it to be suggested as being a useful marker of neural density (Heiss et al., 1998, la Fougère et al., 2011), supported by observations of reduced FMZ binding linked to neuronal loss in cases of brain damage such as stroke (Heiss et al., 1998 ) and the vegetative state (Rudolf et al., 2002). Of course, any such strong correlation between FMZ binding and neural density within regions and across subjects would present a problem for drawing links between FMZ-PET measures and functional imaging results. This is because a low degree of variability between FMZ binding and neural counts between subjects would make a dissociation between the impact of the receptor itself and the impact of simple changes in numbers of neurons on function difficult. As it is aimed here to study the functional profile of the $\mathrm{GABA}_{\mathrm{A}}$ receptor, distinct from neural density, an analysis of the relationship between FMZ binding and GM density was undertaken to clarify the closeness of the relationship between the two across subjects and is reported in the following chapter. The main analysis of the $\mathrm{GABA}_{\mathrm{A}}$ receptors is then reported in Chapter 8. 


\section{Chapter 7}

\section{Grey matter density and $\mathrm{GABA}_{A}$ binding potential show a positive linear relationship across cortical regions}

\subsection{Abstract}

Voxel based morphometry (VBM) is a widely used technique for studying the structure of the brain. Direct comparisons between the results obtained using VBM and the underlying histology are limited, however. To circumvent the problems inherent in comparing VBM data in vivo with tissue samples that must generally be obtained post-mortem, we chose to consider $\mathrm{GABA}_{\mathrm{A}}$ receptors, measured using ${ }^{18} \mathrm{~F}$-flumazenil $\mathrm{PET}$, as non-invasive neural markers to be compared with VBM data. Consistent with previous cortical thickness findings, $\mathrm{GABA}_{\mathrm{A}}$ receptor binding potential $\left(\mathrm{BP}_{\mathrm{ND}}\right)$ was found to correlate positively across regions with grey matter $(\mathrm{GM})$ density. However, at the level of inter-subject relations between $\mathrm{GM}$ and $\mathrm{BP} \mathrm{ND}_{\mathrm{ND}}$, the relationship was less clear, some regions of the brain showing a correlation between the two measures and others showing none. These findings confirm that there is a general positive relationship between MRI-based GM density measures and $G_{A B A}$ receptor $B P_{N D}$ between regions (i.e., regions with more $G M$ tend to have higher $\mathrm{BP}_{\mathrm{ND}}$ ) and extend this finding into the context of VBM. They also suggest that inter-subject variability in $\mathrm{GABA}_{\mathrm{A}}$ receptors may be dissociated from GM densities.

\subsection{Introduction}

Voxel-based morphometry (VBM) is a widely used MRI technique for studying brain structure. It has been used in many different research contexts, including social neuroscience 
(Kanai et al., 2011), memory (Kanai and Rees, 2011), depression (Bora et al., 2011), and Alzheimer's disease (Ferreira et al., 2011). However, the relationship between VBM measures and actual neural structure has not been well defined.

One approach to linking VBM measures and the underlying histology would be to compare in vivo MRI scans with post-mortem histology. However, given the obvious practical issues involved in such an approach, a non-invasive measure of neural structure is required that can then be compared with VBM data. In this context it has been suggested that ${ }^{18} \mathrm{~F}$ Flumazenil PET (Ryzhikov et al., 2005) (FMZ-PET) could be utilised as an indicator for neuronal density and integrity (Heiss et al., 1998, Hammers et al., 2001, la Fougère et al., 2011). This technique allows the imaging of $\mathrm{GABA}_{\mathrm{A}}$ receptors in vivo in humans (Hammers et al., 2003, Salmi et al., 2008) as Flumazenil binds to the benzodiazepine site on the $\mathrm{GABA}_{\mathrm{A}}$ receptor (Sigel, 2002). With the $\mathrm{GABA}_{\mathrm{A}}$ receptor found widely across the human cortex, playing a major role in neural inhibition, the inference can potentially be drawn between $\mathrm{GABA}_{\mathrm{A}}$ receptor density and the density of neurons.

Adapting this approach, la Fougère and colleagues (la Fougère et al., 2011) compared cortical thickness, as obtained through the analysis of MRI images, with GABA $\mathrm{A}$ receptor density, finding a correlation between these measures across the regions studied. This suggests that the link between GABA $_{\mathrm{A}}$ receptors and morphological measures is valid in the context of cortical thickness analyses. It is not clear, however, if these findings can be extended to VBM.

We thus compared $\mathrm{GABA}_{\mathrm{A}}$ receptor density and VBM measures to establish whether this approach can be applied to VBM also. It was hypothesised that receptor density would correlate with VBM measurements across cortical regions. As a second, exploratory, aim we also investigated whether grey matter measurements within individual regions of interest correlated with $\mathrm{GABA}_{\mathrm{A}}$ receptor density within the same region. 


\subsection{Methods}

\subsubsection{Participants}

Twenty-five healthy subjects (10 female; mean age $22.67 \mathrm{yrs}$, range 18-32 yrs) underwent both FMZ-PET and MRI scanning. PET (Montreal Neurological Institute, McGill) and MRI (Unité de neuroimagerie fonctionelle, Université de Montréal) scans took place on different days (mean time between scans $=2.44$ days, range $=1-6$ days). Subjects were screened for psychiatric or neurological disorders, recreational drug use, and depression, the latter using the Beck Depression Inventory-II with a cut-off of four (Beck et al., 1996). All subjects gave their written informed consent and were compensated financially for their participation. Approval was obtained from the ethics committees at both McGill University and the Université de Montréal. Image analyses were carried out using the FSL suite of tools (Smith et al., 2004).

\subsubsection{MRI}

$\mathrm{T}_{1}$-weighted anatomical images were acquired on a 3 Tesla Siemens Trio scanner using a 16-channel headcoil (MPRAGE, resolution $=1 \times 1 \times 1 \mathrm{~mm}^{3}$ ). Anatomical images were processed in accordance with the FSL-VBM pipeline as follows: Anatomical images were brain extracted and segmented into GM, WM, and CSF partial volume images. The GM images were aligned with the MNI template using a non-linear registration and averaged across subjects to produce a study-specific template. A non-linear registration was then performed between the original GM images and this study-specific template. Finally, the registered partial volume images were modulated (to correct for local expansion or contraction) by dividing by the Jacobian of the warp field.

\subsubsection{PET}

FMZ-PET data was acquired using a Siemens ECAT HRRT PET scanner. ${ }^{18}$ F-flumazenil was synthesised as previously described (Massaweh et al., 2009). Head movement was 
minimised with a head-restraining adhesive band. A 6-minute transmission scan $\left({ }^{137} \mathrm{C}\right.$ point source) was first acquired for attenuation correction followed by an intravenous tracer injection (over $60 \mathrm{~s}$ ) of $260.7 \mathrm{MBq}\left( \pm 21.24 \mathrm{SD}\right.$ ) of ${ }^{18} \mathrm{~F}$-Flumazenil. Subjects were instructed to close their eyes and remain awake during the scan.

List-mode data was acquired for a period of 60 minutes and then binned into a series of 26 sequential sinograms of increasing duration, ranging from $30 \mathrm{~s}$ to $5 \mathrm{~min}$. PET data was reconstructed using a 3D OP-OSEM algorithm (10 iterations and 16 subsets) with correction for scatter, random coincidences, attenuation, decay, dead-time, and frame based motion correction (Costes et al., 2009, Hong et al., 2007, Hudson and Larkin, 1994). The resulting images had a resolution of $1.22 \times 1.22 \times 1.22 \mathrm{~mm}^{3}(256 \times 256 \times 207$ voxels $)$. GABA binding potential $\left(\mathrm{BP}_{\mathrm{ND}}\right)$ maps were then calculated according to the simplified reference tissue method, using the cerebral white matter as the reference tissue region (Logan et al., 1996).

The following steps were adopted to minimise partial volume effects: WM and CSF maps (where each voxel has a value of between 0 and 1, representing the estimated proportion of that voxel that can be assigned to the relevant tissue type) were firstly each thresholded at a tissue proportion of 0.95 and used to produce binary masks. The masks were eroded by two voxels to ensure that only the tissue type of interest (and not GM) was covered, and the mean $\mathrm{BP}_{\mathrm{ND}}$ within these WM and CSF regions calculated. The original, non-eroded, WM and CSF maps were then convolved with a $2.5 \mathrm{~mm}$ FWHM Gaussian kernel to simulate the scanner resolution and were multiplied by the mean $\mathrm{BP}_{\mathrm{ND}}$ value for the appropriate tissue (WM or CSF). These WM and CSF $\mathrm{BP}_{\mathrm{ND}}$ maps were then subtracted from the original $\mathrm{BP}_{\mathrm{ND}}$ maps to give GM $\mathrm{BP}_{\mathrm{ND}}$ images corrected for WM and CSF signal spill over. To further reduce partial volume effects the atlas ROIs were eroded to produce a separation between each one (see below). The BP ${ }_{\text {ND }}$ values obtained were broadly comparable to those obtained in other studies (la Fougère et al., 2011, Odano et al., 2009).

$\mathrm{BP}_{\mathrm{ND}}$ images were aligned to the study GM template in a two-step process. Firstly, the GM map in anatomical space was convolved with a $2.5 \mathrm{~mm}$ FWHM kernel to produce a "simulated PET" image. BP $\mathrm{ND}$ images were then linearly aligned to this image. This linear transform was then combined with the previously calculated non-linear anatomicalto-template warp to transfer the $\mathrm{BP}_{\mathrm{ND}}$ images into the template space. Alignment between the $\mathrm{BP}_{\mathrm{ND}}$ and GM images can be seen in Figure 7.1. 


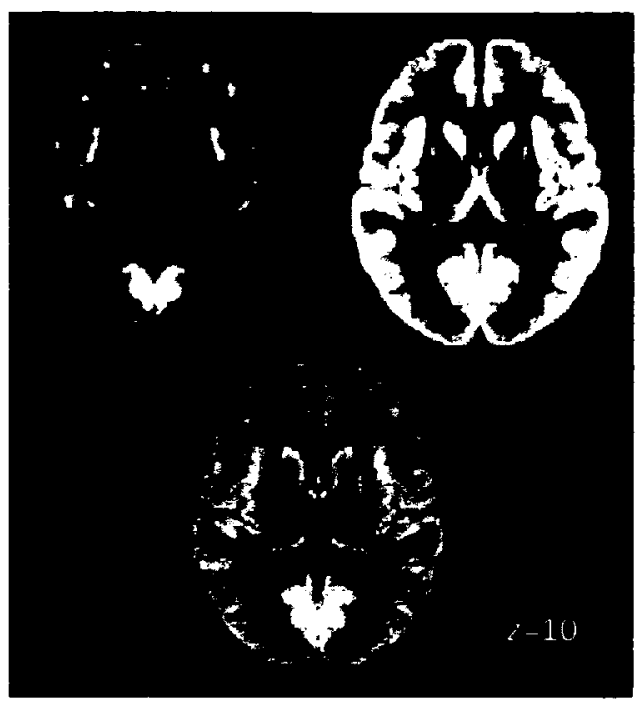

Figure 7.1: Sample BP ND map and GM density image, plus the super-imposition of these to demonstrate alignment between modalities.

\subsubsection{Regions of interest}

Regions of interest were taken from the Jülich histological atlas (http://www. fmrib.ox. ac.uk/fsl/data/atlas-descriptions.html). This atlas consists of 52 grey matter regions (and 10 white matter regions, not included here), bilaterally, and was created using post-mortem crto-architectural segmentations. Following the creation of all 104 ROIs, the masks were eroded by one voxel in order to reduce partial volume effects, the roxel size (2 mm) corresponding reasonably well to the resolution of the PET scamner (FWHMI approx $2.5 \mathrm{~mm}$ ). This erosion also served to reduce the effects of misalignment at the borders of regions. Atlas ROIs were then masked with the study-specific brain template to ensure that no out-of-brain voxels were included in the calculations. ROIs consisting of fewer than 50 voxels after erosion were excluded from the analysis, leaving 59 out of 104 ROIs (see Table 7.1 for list of ROIs included).

The GM volume for each ROI from each subject was extracted from the registered grey matter maps. Mean $\mathrm{BP}_{\mathrm{ND}}$ values were calculated for each $\mathrm{ROI}$ across subjects in the same manner. 


\subsubsection{Comparison of $\mathrm{GM}$ and $\mathrm{BP}$ ND}

Analyses of GMI and BP $P_{N D}$ values were carried out using MATLAB. In a first step, mean $\mathrm{BP}_{\mathrm{ND}}$ and GM values across all regions were compared using partial correlations, controlling for ROI size.

In an exploratory second step, the relationship between $\mathrm{BP}_{\mathrm{ND}}$ and GM was then tested within each individual region. Weighted least squares fits were employed to test for a linear relationship between the two measures, using the inverse variance of each particular ROI's $\mathrm{BP}_{\mathrm{ND}}$ values (i.e., the variance of the values of all voxels within each $\mathrm{ROI}$ for each subject) as a weighting factor to account to some degree for PET measurement error.

\subsection{Results}

\subsubsection{GM and $\mathrm{BP}_{\mathrm{ND}}$ values}

Mean $\mathrm{BP}_{\mathrm{ND}}$ values ranged from 3.7 in the right subiculum of the hippocampus to 9.18 in the right primary visual cortex. Mean GM ranged from 0.34 in the left anterior intra-parietal sulcus to 0.82 in the right entorhinal cortex. Details for all regions can be found in Table 7.1. No significant difference in mean $\mathrm{BP}_{\mathrm{ND}}$ or mean $\mathrm{GM}$ values between hemispheres was observed (paired t-test, $\mathrm{BP}_{\mathrm{ND}} p=0.57, \mathrm{GM} \mathrm{p}=0.64$ ). There were no correlations between ROI size and mean $\mathrm{BP}_{\mathrm{ND}}(\mathrm{R}=0.096, p=0.45,95 \% \mathrm{CI}=-0.16-0.39)$ or mean $\mathrm{GM}(\mathrm{R}$ $=-0.06, p=0.67,95 \% \mathrm{CI}=-0.33-0.21)$.

\subsubsection{Global BP $\mathrm{ND}_{\mathrm{G}} \mathrm{GM}$ correlation}

A scatter-plot of the $\mathrm{BP}_{\mathrm{ND}}$ and $\mathrm{GM}$ data revealed two distinct groupings of regional values (Figure 7.2). The larger (51 ROIs) of these consisted of mainly cortical regions, whilst the smaller (8 ROIs) consisted of more subcortical regions (amygdala and hippocampus)(see Table 7.1). Separate correlations were carried out for each sub-group. In the larger group, mean $\mathrm{BP}_{\mathrm{ND}}$ and mean GM across regions were positively correlated $\left(\mathrm{R}=0.69, p_{\mathrm{FDR}}<\right.$ 


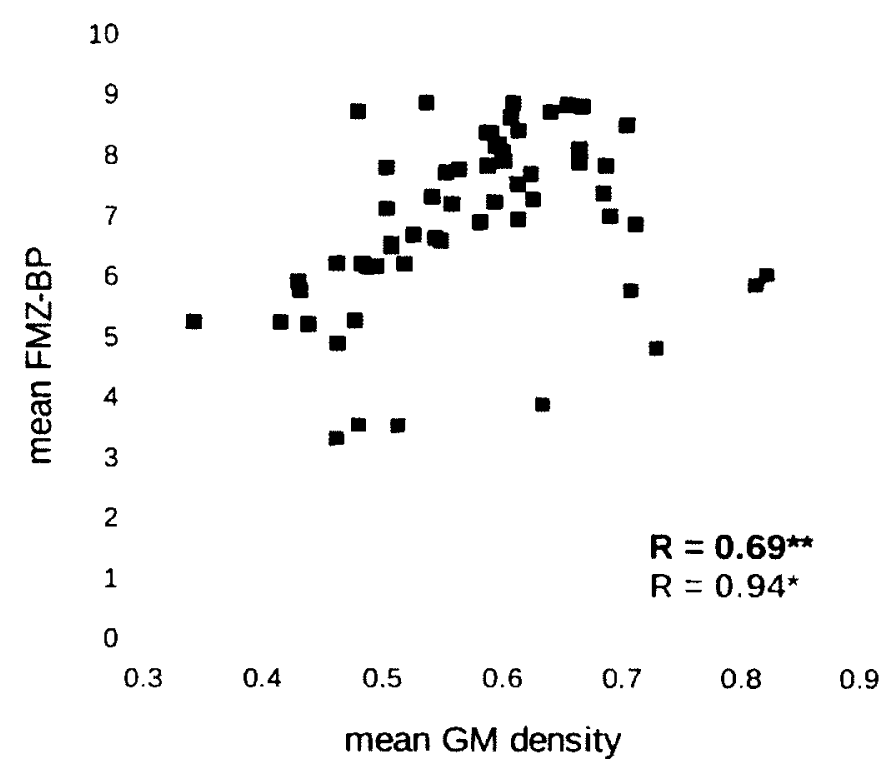

Figure 7.2: Correlation between mean BP ND and mean GM in each of the two identified groupings of ROIs. Blue points correspond to cortical regions (see Table 1 for full list). A linear relationship between mean $\mathrm{BP}_{\mathrm{ND}}$ and mean GM can be seen. Green points correspond to regions of the hippocampus and amygdala. * indicates $p_{\mathrm{FDR}} \cdot 0.05,{ }^{* *}$ indicates $p_{\mathrm{FDR}} \cdot 0.001$.

$0.0001,95 \%$ CI $-0.52-0.82)$. This was also the case in the smaller group $\left(\mathrm{R}=0.94, p_{\mathrm{FDR}}\right.$ $=0.018,95 \% \mathrm{CI}=0.69 \cdot 0.99)$.

\subsubsection{Regional BP ND $_{\text {-GM correlation }}$}

A positive correlation between $\mathrm{BP}_{\mathrm{ND}}$ and $\mathrm{GM}$ was seen in regions of the right anterior int raparietal sulcus, the right amygdala, bilateral hippocampus, and right V3. It is worth noting also that a trend to significance $(p, 0.1)$ was seen in several additional regions, including the left intra-parietal sulcus and left $\backslash 3$. Whilst the majority of significant relationships were positive, some regions displayed a negative correlation between the two measures, including sections of the inferior parital lobule and secondary somatosensory cortex (see Table 7.1 for details and Supplementary figure C.1a\&b for plots). 


\begin{tabular}{|c|c|c|c|c|c|c|c|c|}
\hline & Vol. & $B P_{N D}$ & GM & Vol. & $\mathrm{BP}_{\mathrm{ND}}$ & GM & \multicolumn{2}{|c|}{ BP NDvs G.M } \\
\hline Ant. IPS 1 & 125 & $5.64 \pm 0.98$ & $0.45 \pm 0.08$ & 84 & $5.36 \pm 1.08$ & $0.46 \pm 0.12$ & $1.57 \dagger$ & $2.72^{*}$ \\
\hline Ant. IPS 3 & 58 & $5.71 \pm 1.11$ & $0.34 \pm 0.10$ & - & - & - & $1.24 \dagger$ & - \\
\hline Broca's (BA44) & 1480 & $7.71 \pm 0.87$ & $0.55 \pm 0.08$ & 853 & $8.37 \pm 1.08$ & $0.6 \pm 0.08$ & 0.11 & 0.07 \\
\hline Broca's (BA45) & 1224 & $8.16 \pm 1.05$ & $0.60 \pm 0.09$ & 1148 & $7.38 \pm 1.02$ & $0.50 \pm 0.07$ & 0.97 & 0.16 \\
\hline IPL PF & 367 & $8.66 \pm 1.00$ & $0.61 \pm 0.10$ & 328 & $8.05 \pm 1.31$ & $0.69 \pm 0.1$ & $-1.73^{*}$ & $-1.46 \dagger$ \\
\hline IPL PFcm & 86 & $7.32 \pm 0.76$ & $0.61 \pm 0.19$ & - & - & 一 & -1.17 & - \\
\hline IPL PFm & 75 & $8.86 \pm 1.04$ & $0.61 \pm 0.12$ & 237 & $8.14 \pm 0.95$ & $0.66 \pm 0.10$ & -0.89 & -0.75 \\
\hline IPL Pga & 164 & $8.42 \pm 1.00$ & $0.59 \pm 0.11$ & 433 & $8.34 \pm 0.94$ & $0.66 \pm 0.10$ & 0.66 & $-1.71^{*}$ \\
\hline IPL PGp & 796 & $8.08 \pm 0.98$ & $0.59 \pm 0.08$ & 822 & $8.28 \pm 0.99$ & $0.60 \pm 0.08$ & 0.01 & -0.16 \\
\hline Premotor & 4285 & $7.19 \pm 0.93$ & $0.58 \pm 0.07$ & 4218 & $6.90 \pm 0.87$ & $0.55 \pm 0.05$ & -0.51 & -0.53 \\
\hline $1^{\circ}$ Motor & 178 & $6.44 \pm 0.91$ & $0.49 \pm 0.10$ & 136 & $6.18 \pm 0.91$ & $0.43 \pm 0.07$ & 0.05 & -0.49 \\
\hline $1 \cdot \mathrm{SS}(\mathrm{BA} 1)$ & 175 & $6.89 \pm 1.16$ & $0.54 \pm 0.09$ & 133 & $5.46 \pm 1.37$ & $0.48 \pm 0.08$ & 0.28 & -0.84 \\
\hline $1^{\circ} \mathrm{SS}(\mathrm{BA} 2)$ & 92 & $8.10 \pm 1.02$ & $0.50 \pm 0.12$ & 94 & $7.62 \pm 1.19$ & $0.54 \pm 0.09$ & 0.72 & 0.72 \\
\hline $2^{*} \mathrm{SS} O P 1$ & 171 & $8.97 \pm 1.02$ & $0.64 \pm 0.11$ & 133 & $9.06 \pm 1.20$ & $0.65 \pm 0.10$ & $-1.55 \dagger$ & 0.36 \\
\hline $2^{\circ}$ SS OP 3 & 121 & $7.51 \pm 0.77$ & $0.59 \pm 0.08$ & 140 & $7.00 \pm 0.87$ & $0.53 \pm 0.07$ & -0.93 & -1.21 \\
\hline $2^{*}$ SS OP4 & 183 & $7.82 \pm 0.97$ & $0.61 \pm 0.11$ & 156 & $6.50 \pm 1.01$ & $0.52 \pm 0.11$ & -0.55 & -1.01 \\
\hline SPL $5 \mathrm{Ci}$ & 54 & $8.46 \pm 1.17$ & $0.60 \pm 0.12$ & 123 & $8.64 \pm 1.06$ & $0.59 \pm 0.10$ & $1.43 \dagger$ & 0.53 \\
\hline SPL 5L & 65 & $6.78 \pm 1.24$ & $0.51 \pm 0.11$ & 97 & $5.93 \pm 1.19$ & $0.43 \pm 0.12$ & 0.36 & -0.03 \\
\hline SPL 5M & 110 & $9.11 \pm 1.18$ & $0.61 \pm 0.09$ & 56 & $8.05 \pm 1.21$ & $0.56 \pm 0.12$ & -0.68 & $-1.42 \dagger$ \\
\hline SPL 7A & 891 & $6.48 \pm 0.90$ & $0.48 \pm 0.07$ & 393 & $6.74 \pm 0.87$ & $0.51 \pm 0.09$ & -0.94 & -0.80 \\
\hline SPL $7 \mathrm{M}$ & - & - & - & 70 & $9.06 \pm 1.00$ & $0.67 \pm 0.11$ & - & $1.68 \dagger$ \\
\hline SPL 7P & 228 & $5.43 \pm 1.02$ & $0.41 \pm 0.07$ & 349 & $6.38 \pm 0.99$ & $0.49 \pm 0.10$ & 0.85 & -1.10 \\
\hline V1 & 513 & $9.00 \pm 1.19$ & $0.48 \pm 0.07$ & 783 & $9.18 \pm 1.18$ & $0.54 \pm 0.09$ & -0.45 & 1.23 \\
\hline $\mathrm{V} 2$ & 405 & $6.46 \pm 0.92$ & $0.46 \pm 0.06$ & 711 & $7.50 \pm 0.83$ & $0.56 \pm 0.08$ & -0.32 & $1.38 \dagger$ \\
\hline V3 & 70 & $7.63 \pm 0.90$ & $0.62 \pm 0.11$ & 89 & $7.32 \pm 1.03$ & $0.62 \pm 0.11$ & $1.67 \dagger$ & $2.51^{*}$ \\
\hline V4 & 481 & $7.66 \pm 0.82$ & $0.68 \pm 0.07$ & 416 & $7.14 \pm 0.92$ & $0.71 \pm 0.11$ & -0.38 & 0.67 \\
\hline V5 & 285 & $8.00 \pm 0.82$ & $0.62 \pm 0.13$ & 315 & $8.73 \pm 1.10$ & $0.62 \pm 0.13$ & -0.24 & 0.93 \\
\hline Amyg. LBG & 56 & $5.00 \pm 0.85$ & $0.73 \pm 0.06$ & 62 & $3.85 \pm 0.56$ & $0.51 \pm 0.06$ & $1.60 \dagger$ & $1.88^{*}$ \\
\hline Hippo. CA & 111 & $6.00 \pm 0.76$ & $0.81 \pm 0.09$ & 134 & $4.15 \pm 0.59$ & $0.63 \pm 0.08$ & $1.71 \dagger$ & $2.48^{*}$ \\
\hline Hippo. EC & 334 & $5.91 \pm 0.73$ & $0.71 \pm 0.15$ & 402 & $6.17 \pm 0.66$ & $0.82 \pm 0.16$ & 0.80 & 0.88 \\
\hline Hippo. Sub & 90 & $3.90 \pm 0.66$ & $0.48 \pm 0.10$ & 112 & $3.70 \pm 0.57$ & $0.46 \pm 0.08$ & $2.63^{*}$ & $1.92^{*}$ \\
\hline
\end{tabular}

Table 7.1: ROIs used, with ROI volume (voxels), and mean $B_{N D}$ and GM values ( \pm S.D.) for the left and right hemisphere. Also shown are t-statistics for the slope of the weighted linear fit between within-region BP ND and GM (* indicates $p<0.05 ; \dagger$ indicates $p<0.1$ ). Amyg. = amygdala, Ant. IPS = anterior intra-parietal sulcus, Hippo. $=$ hippocampus, $\mathrm{IPL}=$ inferior parietal lobule, $1^{\circ}$ Motor $=$ primary motor cortex, Premotor $=$ premotor cortex, $1^{\circ} \mathrm{SS}=$ primary somatosensory cortex $2^{\circ} \mathrm{SS}=$ secondary somatosensory cortex, $\mathrm{SPL}=$ superior parital lobule, $\mathrm{V}=$ visual cortex. 


\subsection{Discussion}

Using a combination of high resolution FMZ-PET and VBM, we investigated the relationship between $\mathrm{GABA}_{\mathrm{A}}$ receptor density and $\mathrm{GM}$ density in regions of the cortex defined according to a histological atlas. $\mathrm{GABA}_{\mathrm{A}} \mathrm{BP}_{\mathrm{ND}}$ was found to correlate with GM density across regions. This finding echoes previous findings that show a correlation between $\mathrm{GABA}_{\mathrm{A}}$ receptor density and cortical thickness across regions (la Fougère et al., 2011). The direction of the correlation is reversed in VBM as compared to cortical thickness, however, with a positive correlation compared to a negative one, respectively. To explain this we can consider the nature of the two measurements. VBM gives information as to the volume of grey matter that is present in a region: The positive correlation thus represents the relationship between the total volume of GM and the number of receptors to bind with more GM there are more receptors. In contrast, and as discussed by la Fougère and colleagues, cortical thickness could be described as giving a measure of the distribution of the grey matter within a region - with greater cortical thickness there is more space, so to speak, in which to distribute neurons and hence lower densities (although this will be variable across different regions Collins et al. (2010)). With greater cortical thickness, and hence lower density, one would then expect a lower $\mathrm{BP}_{\mathrm{ND}}$ value within a specific region. This can be illustrated by taking the primary visual cortex as an example. In the present study this region was found to have both a large GM density and high BP (see Table 7.1), whilst la Fougère and colleagues observed high $\mathrm{BP}_{\mathrm{ND}}$ but low cortical thickness (i.e., high GM density).

Taking each of the Jülich atlas regions separately, a positive linear relationship between $\mathrm{BP}_{\mathrm{ND}}$ and $\mathrm{GM}$ was seen in some regions, although other regions showed no effect. A number of regions displayed a negative relationship (see Table 7.1) This apparent complexity of relationships between $\mathrm{BP}_{\mathrm{ND}}$ and GM across different regions (which must, however, be treated as tentative due to potential confounding factors - see limitations, below) is in agreement with the results of the one prior study directly comparing within-region VBM measures with histology (Eriksson et al., 2009). This study involved temporal lobe epilepsy patients undergoing surgical ablation of the seizure focus - the temporal lobe was scanned and a standard VBM analysis carried out before the anterior temporal lobe was then surgically removed and the neuronal density directly calculated. No correlation was found between MRI measures of GM and actual neuronal density, nor with a range of other 
histopathological measures. The apparent variability across regions of the relationship between regional F.MZ-PET and GMI is also in accordance with the plasticity of the GABAA receptor (Arancibia-Carcamo and Kittler, 2009, Vithlani et al., 2011), as well as with prior work showing that the functional state of GABA-ergic neurons (i.e., the release and uptake of the transmitter during activity) alters the affinity of the benzodiazepine site to which flumazenil binds (Frankle et al., 2012, Miller et al., 1988).

Some limitations of the current study must be considered. Firstly, PET and MRI scans were carried out on different days for practical reasons. As the retest reliability of binding potentials calculated using the simplified reference tissue approach adopted here is not ideal (Salmi et al., 2008) (although it should be noted that the data used in Salmi et al. was acquired using ${ }^{11} \mathrm{C}$-flumazenil rather than ${ }^{18} \mathrm{~F}$-flumazenil), this may introduce some error into the measurements. Replication using data acquired more closely together is required. Secondly, as the histological atlas used does not cover the whole brain, there may be differences in results when a segmentation of the entire cortex is used. An analysis of this dataset using the whole-brain Harvard-Oxford atlas (http://www.cma.mgh.harvard. edu/fsl_atlas.html) produced analogous results to those obtained with the Jülich atlas (inter-regional correlation, $\mathrm{R}=0.44, \mathrm{p}<0.0001,95 \% \mathrm{CI}=0.22-0.56$ ); however, as there must be questions regarding the closeness of match between such regional atlases and the underlying cortical organisation (Craddock et al., 2011), the possibility must remain open that different results would be found with a whole-brain histological atlas. Finally, as described above, the lack of correlation within individual regions may be due to the noise level in the data at the subject level being too high. The reasonable subject number (n $=25$ ) and high resolution data, coupled with the use of weighting to try and account for this factor, would suggest that this is not the case, but replication with a larger sample is warranted.

To conclude, using a combination of VBM and ${ }^{18} \mathrm{~F}$-flumazenil PET it was shown that there is a general positive relationship between $\mathrm{GABA}_{\mathrm{A}} \mathrm{BP}_{\mathrm{ND}}$ and $\mathrm{GM}$ across histologically delineated regions of the brain. A more complex relationship between regional FMZ-PET and GM at the subject level was, however, suggested by there being a linear relationship between the two measures in some but not all regions studied. This complex relationship may reflect functionally relevant variation in $\mathrm{GABA}_{\mathrm{A}}$ receptors between subjects. 


\subsection{Acknowledgements}

Many thanks to K. Dedovic and A. Perna for their invaluable help with subject recruitment, as well the staff at the MNI and UNF for their assistance. The study was supported by grants to G.N. from the Canadian Institutes of Health Research and Hope for Depression Research Foundation. 


\section{Chapter 8}

\section{Cortical $\mathrm{GABA}_{\mathrm{A}}$ receptor binding potential patterns display small-world characteristics}

\subsection{Abstract}

Neuroimaging has revealed a picture of the brain as a massively connected network, giving insight into its functional and structural organisation and specialisations. More recently, graph theoretical approaches have been applied to the brains structural and functional connectivity. However, the link between these domains remains indirect. In order to provide a bridge between them, ${ }^{18} \mathrm{~F}$-flumazenil $\mathrm{PET}$ was used to map $\mathrm{GABA}_{\mathrm{A}}$ receptor binding potential $\left(\mathrm{BP}_{\mathrm{ND}}\right)$ in healthy humans. In line with structural and functional networks, it was found that the regional $\mathrm{BP}_{\mathrm{ND}}$ connectivity network followed a small-world organisation. In addition, the overall network was found to be composed of four sub-modules, including a visual module and a midline-insular module. These findings thus provide information as to the organisation of $\mathrm{GABA}_{\mathrm{A}}$ receptors in the human brain and provide a potential link between structure and function.

\subsection{Introduction}

The brain can be described as a massively connected network. Neuroimaging has proved a productive approach for classifying the functional configurations and properties of this network that underlie particular processes, such as reward (Liu et al., 2011), aversion (Hayes and Northoff, 2011), and empathy (Fan et al., 2011). Similarly, imaging has been used to identify intrinsic activity networks that are present even in the absence of any particular 
stimulus (Fox and Raichle, 2007, Raichle, 2009). In addition, brain networks have been described at the level of structural connections (Achard et al., 2006, He et al., 2007, Hagmann et al., 2008), with such anatomical networks corresponding to a large extent to those seen at a functional level (Honey et al., 2008, 2009) (although with potentially important divergence (Sadaghiani et al., 2010)).

More recently, graph theoretical approaches have been applied to brain networks to describe their structure and the transfer of information across them (Bullmore and Bassett, 2011, Rubinov and Sporns, 2010). Graph theoretical analyses stem from the mathematical investigation of the inter-relations between members of a particular group of entities, where each entity is defined as a "node" and the links between them as "edges", and have been applied to a wide range of abstract and practical applications (Barabási, 2009). Using such approaches, it has been found that the anatomical and functional networks in the brain are organised according to what has been termed a "small-world" arrangement. This means that regions that are close to each other have dense connections whilst at the same time efficient connections between different such local clusters exist. This combination of local clustering and efficient long range connections is thought to provide an optimal balance between the need for local areas to functionally specialise and the need for the brain as a whole to integrate as a network (Rubinov and Sporns, 2010). At the same time, particular regions have been identified as being particularly important for the transfer of information across the brain network, generally being located in the posterior cortex around the cortical midline (Achard et al., 2006, Hagmann et al., 2008, van den Heuvel and Sporns, 2011).

However, whilst an increasing amount is known about structural and functional networks, far less is known about the comparable organisation of the receptors for the neurotransmitters. As such receptors could be said to represent a link between the structural and functional domain, and so knowledge at this level may provide important insights into brain function. Of the various receptor types in the human cortex, the GABAA receptor - the primary inhibitory receptor - is particularly widely distributed (Jacob et al., 2008, Olsen, 1981), making it a promising candidate for receptor network investigations. We therefore employed radiolabelled flumazenil PET (FMZ-PET) to measure whole-brain $\mathrm{GABA}_{\mathrm{A}}$ receptor distributions in vivo in healthy humans (Frankle et al., 2012, Odano et al., 2009, Salmi et al., 2008). This data was used to create a $\mathrm{GABA}_{\mathrm{A}}$ receptor connectivity matrix upon which standard graph-theory measures were applied. It was predicted that, in line with functional and structural brain networks, the GABA $\mathrm{A}$ connectivity network 
would display small-world characteristics (Bullmore and Bassett, 2011). In addition, it was predicted that network properties of $\mathrm{GABA}_{\mathrm{A}}$ receptor connectivity, such as network modularity and the location of hubs, would mirror to a large extent those reported for functional and structural networks in the human brain.

\subsection{Methods}

\subsubsection{Participants}

Twenty-five healthy subjects (10 female; mean age $22.67 \mathrm{yrs}$, range $18-32$ yrs) underwent both PET and MRI scanning. PET (Montreal Neurological Institute, McGill) and MRI (Unité de neuroimagerie fonctionelle, Université de Montréal) scans took place on different days (mean time between scans $=2.44$ days, range $=1-6$ days). Subjects were screened for psychiatric or neurological disorders, as well as for heavy recreational drug use (including alcohol). All subjects gave their written informed consent and were compensated financially for their participation. Approval was obtained from the ethics committees at both McGill University and the Université de Montréal. Image analyses were carried out using the FSL suite of tools (Smith et al., 2004).

\subsubsection{PET}

FMZ-PET data was acquired using a Siemens ECAT HRRT PET scanner. ${ }^{18} \mathrm{~F}$-flumazenil was synthesised as previously described (Massaweh et al., 2009). Head movement was minimised with a head-restraining adhesive band. A 6-minute transmission scan $\left({ }^{137} \mathrm{C}\right.$ point source) was first acquired for attenuation correction followed by an intravenous tracer injection (over $60 \mathrm{~s}$ ) of $260.7 \mathrm{MBq}\left( \pm 21.24 \mathrm{SD}\right.$ ) of ${ }^{18} \mathrm{~F}$-flumazenil. Subjects were instructed to close their eyes and remain awake.

List-mode data was acquired for a period of 60 minutes and then binned into a series of 26 sequential sinograms of increasing duration, ranging from $30 \mathrm{~s}$ to $5 \mathrm{~min}$. PET data was 


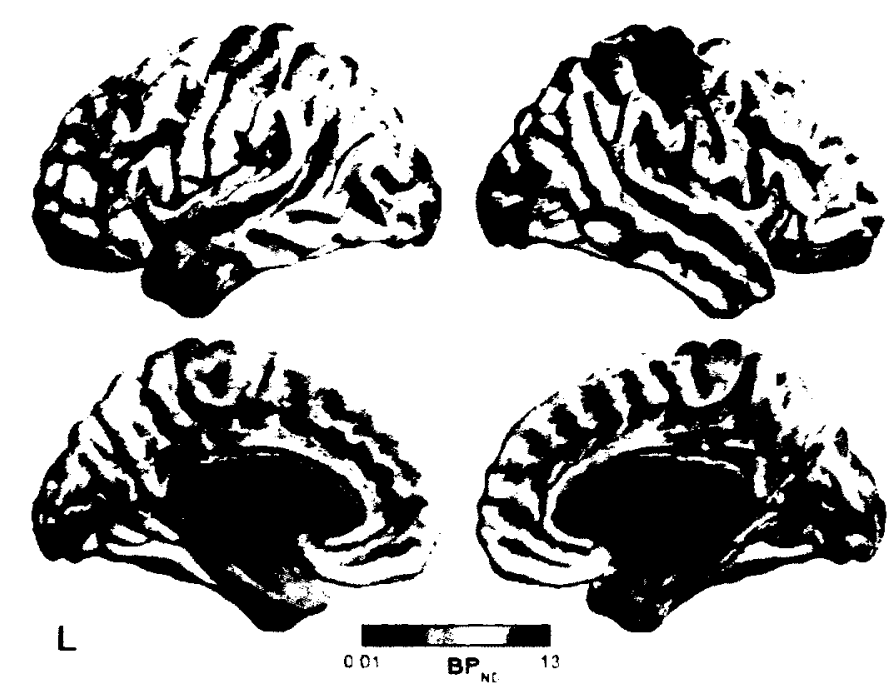

Figure 8.1: Cortical GABA $\mathrm{BP}_{\mathrm{ND}}$ distribution. Image represents mean BP $\mathrm{ND}$ across all subjects aligned to MNI spare. Greater BPND approximates to greater receptor density:

reconstructed using a 3D OP-OSEM algorithm (10 iterations and 16 subsets) with correction for scatter, random coincidences, attenuation, decay, dead-time, and frame based motion correction (Costes et al., 2009, Hong et al., 2007, Hudson and Larkin, 1994). The resulting images had a voxel size of $1.22 \times 1.22 \times 1.22 \mathrm{~mm}^{3}(256 \times 256 \times 207$ voxels $)$. GABA binding potential $\left(\mathrm{BP}_{\mathrm{ND}}\right)$ maps were then calculated according to the simplified reference tissue model, using the cerebral white matter as the reference tissue region (Logan et al., 1996) (see Figure 8.1 for cortical $\mathrm{BP}_{\mathrm{ND}}$ distribution).

\subsubsection{MRI}

$\mathrm{T}_{1}$-weighted anatomical images were acquired with a 3 Tesla Siemens Trio scanner using a 16-channel headcoil (MPRAGE: FoV $=205 \times 205 \mathrm{~mm}^{2}$ : spatial resolution - $1 \times 1 \times 1$ $\mathrm{mm}^{3}: \mathrm{T}_{\mathrm{E}}-3.02 \mathrm{~ms}: \mathrm{T}_{\mathrm{R}}-2000 \mathrm{~ms}$; flip angle $\left.-5^{\circ}\right)$.

Anatomical images were aligned to the MNI standard-space template provided with FSL. Non-linear alignments were calculated using the FNIRT tool. PET images were aligned to 
the same standard-space template through a multi-step process. Firstly, anatomical images were segmented into grey-matter, white-matter, and cerebrospinal fluid. Grey matter maps were convolved with a $2.5 \mathrm{~mm}$ FWHM Gaussian kernel (reflecting the resolution of the PET scanner) to produces 'simulated' PET images. The BP ND images were then linearly aligned to this 'simulated' image and the transformation matrices from this step combined with the anatomical-to-MNI warps previously calculated to create a PET-to-MNI transformation.

\subsubsection{Network creation}

$\mathrm{T}_{1}$-weighted anatomical images were segmented into 68 individually defined cortical regions of interest (ROIs) using Freesurfer (Desikan et al., 2006, Fischl et al., 2002, 2004). These were transformed into the MNI standard space using the previously calculated transformations. The mean $\mathrm{BP}_{\mathrm{ND}}$ values from each ROI were then extracted for each subject. In the same way, mean grey-matter densities for each ROI were extracted from the grey-matter density maps produced previously. $\mathrm{BP}_{\mathrm{ND}}$ values for each $\mathrm{ROI}$ were then corrected across subjects for potentially confounding variables by regressing out subject age, sex, total brain volume, whole-brain mean $\mathrm{BP}_{\mathrm{ND}}$ values, and ROI grey matter density.

Using these corrected values, a connectivity matrix was created. This was done by calculating the linear correlation between $\mathrm{BP}_{\mathrm{ND}}$ values across subjects for all ROIs, resulting in a $68 \times 68$ matrix. This matrix was then binarised, firstly by assigning a value of one to significant correlations and zero to all others using a conservative significance threshold of $p<0.05$, FDR corrected for multiple comparisons (He et al., 2007), and secondly in an process that created a range of graphs with sparsities (i.e. the fraction of connections that are retained out of all connections) that ranged from $14-26 \%$ in increments of $1 \%$. Graph measures were calculated at each sparsity and integrated across them. This latter step was employed as the level of sparsity influences the graph properties, but as thresholding is to some extent arbitrary, it is possible that 'true' network properties could be missed at any one level. Integrating over a range of thresholds thus improves the robustness of the network characterisation (Dennis et al., 2012). The range of $14-26 \%$ sparisty was chosen as it is both biologically plausible (Dennis et al., 2012) and contains the sparsity of the conservative FDR thresholded graph that represents statistically significant connections between regions (16\% - see Results). In the resulting graphs, each node thus represented a brain region and each edge the correlation of $B P_{N D}$ values between regions. 
As well connectivity between regions, the anatomical distances (in $\mathrm{mm}$ ) between ROI centres were calculated as $D_{i j}=\sqrt{\left(x_{\mathrm{i}}-x_{\mathrm{j}}\right)^{2}+\left(y_{\mathrm{i}}-y_{\mathrm{j}}\right)^{2}+\left(z_{\mathrm{i}}-z_{\mathrm{j}}\right)^{2}}$, where $D_{i j}$ is the distance between two ROIs, $i$ and $j$, and $x, y$, and $z$ represent the Euclidean coordinates of the ROIs (Achard et al., 2006, He et al., 2007). The likelihood of a connection between regions in the single FDR thresholded graph was compared to the distances between them by calculating the frequency of significant connectivity for any particular distance, binned in $10 \mathrm{~mm}$ increments.

\subsubsection{Network metrics}

A range of graph theory metrics were calculated for the 68 node unweighted and undirected graphs resulting from the preceding steps. The Brain Connectivity Toolbox (https:// sites.google.com/site/bctnet/) was used for all analyses (Rubinov and Sporns, 2010). The measures calculated for the graph as a whole were the mean clustering coefficient, characteristic path length and global efficiency. Clustering coefficient is a measure of the extent to which nodes cluster together, meaning that graphs that have a large coefficient have a lot of connections between regions that are close to each other. Path length represents the average number of connections that must be traversed in order to get from any one region to another.

In addition to these global metrics, degree and betweenness centrality were calculated at the level of individual nodes (Bullmore and Bassett, 2011, Rubinov and Sporns, 2010). The degree of a particular node is equal to the number of edges that originate from it, meaning that a region with numerous connections to other regions will have a high degree. Betweenness centrality represents how many of the shortest paths across the network must pass through a particular node. This means that a region that has a high betweenness centrality plays an important role in connections between a large number of other regions. Mathematical definitions of each measure can be found in (Rubinov and Sporns, 2010).

The small-worldness of the network was tested by calculating its small-world index $(\sigma)$. This is defined as the mean clustering coefficient normalised relative to that of a random graph $(\gamma)$ divided by the characteristic path length, also normalised relative to that of a random graph $(\lambda) .100$ random graphs with the same number of nodes and edges as the $B P_{N D}$ graphs were created for the comparisons and the average $\gamma$ and $\lambda$ across all used. A $\sigma$ 
greater than one resulting from a $\gamma$ that is also greater than one and a $\lambda$ of approximately one is generally taken to denote a small-world network (Bullmore and Bassett, 2011, Humphries et al., 2006).

Finally, the overall network was split into sub-networks by calculating the partition whereby the ratio between connections within sub-networks compared to connections between subnetworks was maximal (Newman, 2006). The intra-modular degree and participation coefficient for the sub-networks were also calculated (Guimerà et al., 2007). Intra-modular degree is a measure of how many connections a node makes to other nodes within its module. A region with a high intra-modular degree would thus be highly connected within the module it is a part of. In contrast, the participation coefficient is calculated as the ratio between the number of connections that a node makes within its module to the number of connections it makes to other modules. A region with a high participation would thus be important for information transfer between modules, as opposed to information transfer within modules (where a region with a high intra-modular degree would be important).

\subsection{Results}

Using $\mathrm{p}_{F D R}<0.05$ as the threshold, significant connectivity was observed between 358 pairs of regions (Figure 8.2), producing a connectivity matrix with a sparsity of approximately $16 \%(411 / 2278)$. As with previous studies of brain networks, the likelihood of a connection being found decreased with increasing Euclidean distance between regions, following an apparent exponentially truncated power distribution (Figure 8.2) (He et al., 2007). The FDR-thresholded network had a sigma of 1.74 , whilst the integrated sigma value was 1.86 (Table 8.1), values that are comparable to anatomical and functional studies (Achard and Bullmore, 2007, He et al., 2007, van den Heuvel and Sporns, 2011). This indicates that the distribution of $\mathrm{GABA}_{\mathrm{A}}$ receptors in the brain follows a small-world model.

Node-specific metrics - degree and betweenness centrality - are displayed in Figure 8.3.More nodes of a high degree (greater than the mean $+1 \mathrm{SD}$ ) were located in the left hemisphere. In both hemispheres, high degree nodes were concentrated in the frontal and temporal cortices. In contrast, nodes with high betweenness centrality were concentrated along the cortical midline. 

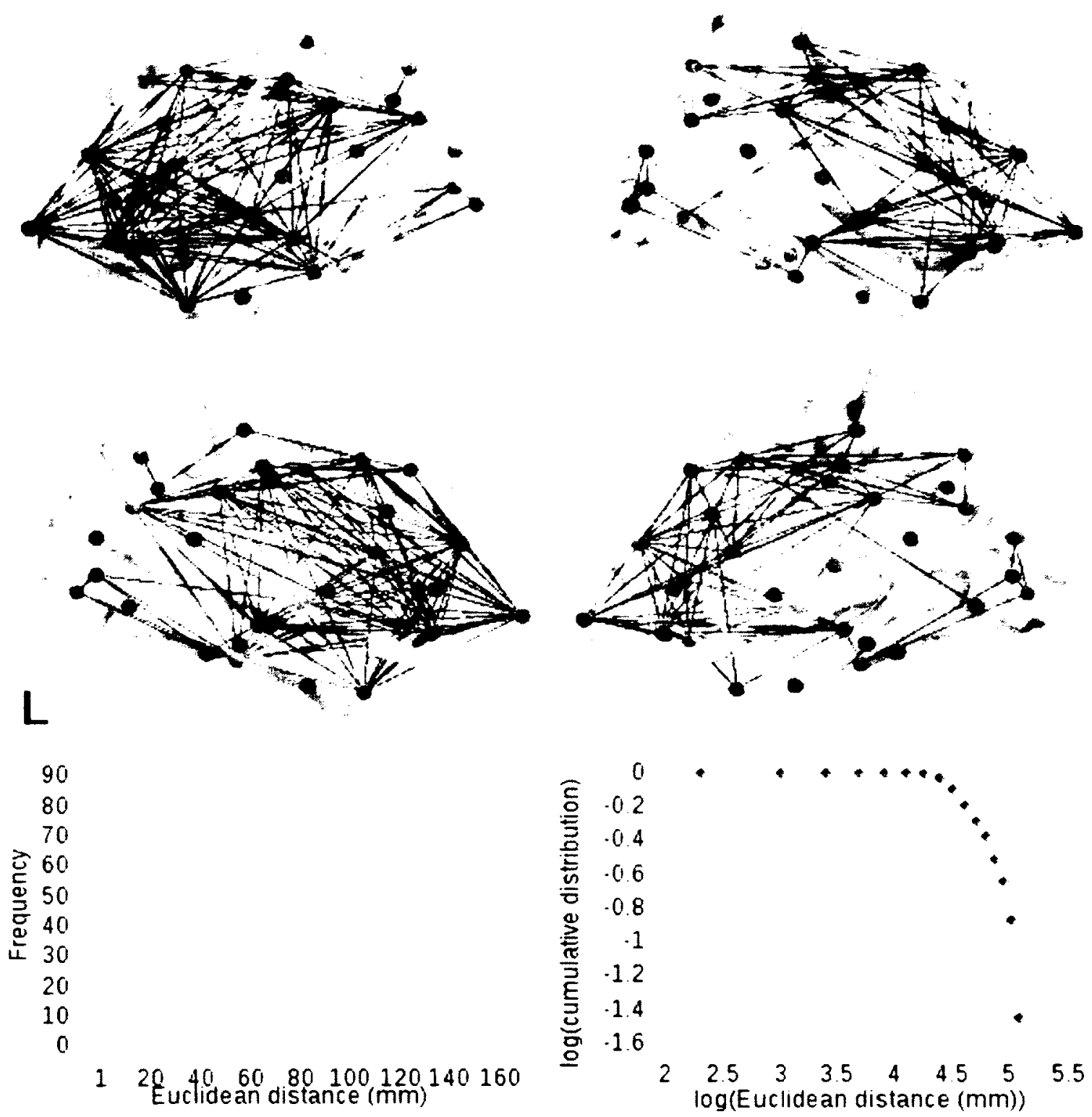

Figure 8.2: Connection between all nodes in the FDR-corrected graph and the dependence on distance of connections between nodes. Frequency value represents number of connections over a given distance. This probability reduces with increasing distance according to an apparent truncated power distribution, indicating a sinall-world topology: 


\begin{tabular}{cccccc} 
& $\mathrm{CC}$ & $\mathrm{L}$ & $\gamma$ & $\lambda$ & $\sigma$ \\
\hline FDR & 0.56 & 2.55 & 1.41 & 0.83 & 1.74 \\
Integrated & 0.68 & 2.70 & 1.64 & 1.06 & 1.86
\end{tabular}

Table 8.1: Small world properties for FDR-corrected and integrated graphs. CC - clustering coefficient, L characteristic path length. $\gamma$ normalised clustering coefficient, $\lambda$ normalised path length, $\sigma-$ small world index.

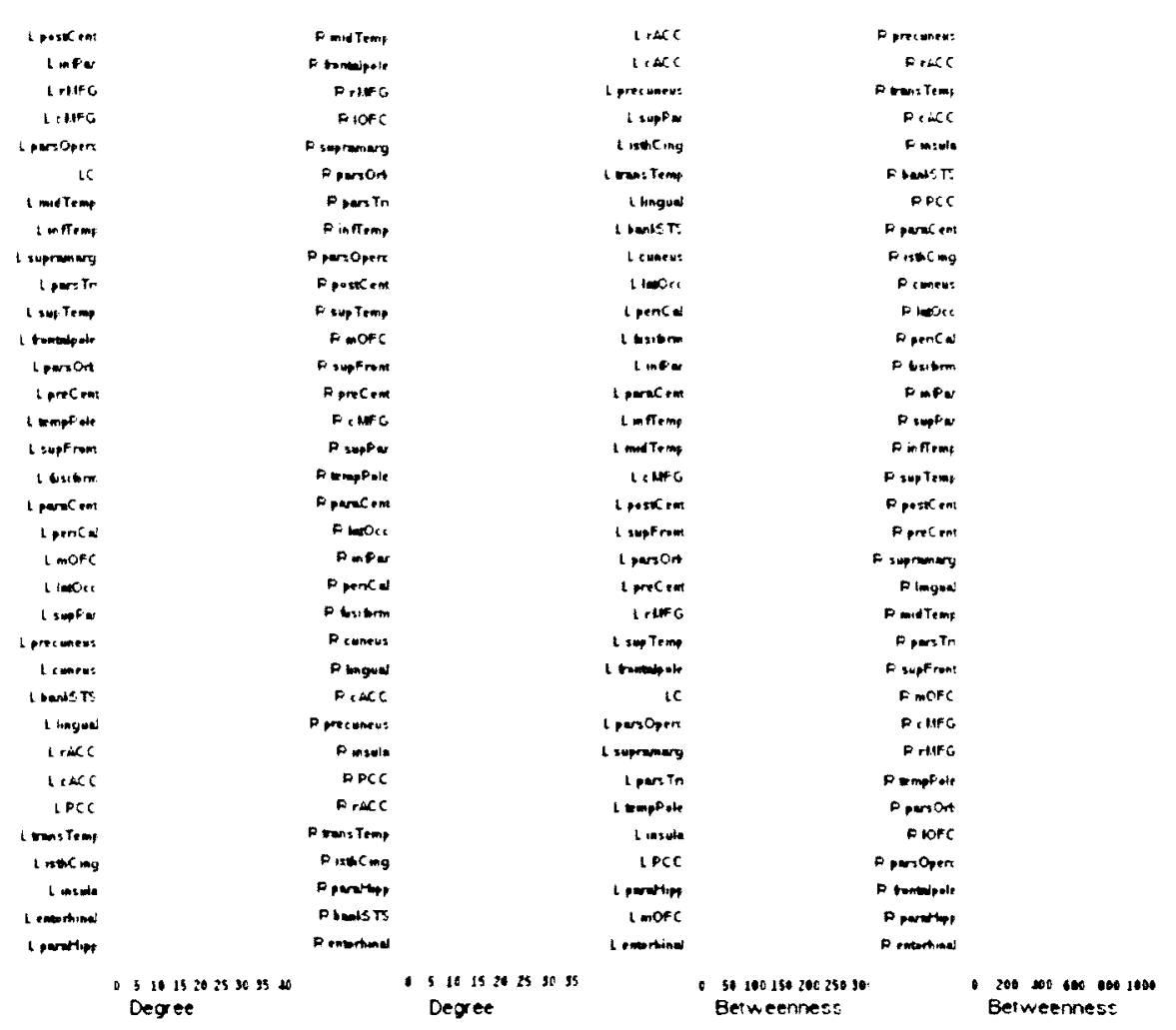

Figure 8.3: Degree and betweemess centrality for each node. Those with high values (mean - $1 \mathrm{SD}$ ) are indicated in red. These nodes represent hubs in the graph and are of particular importance to information transfer across it. 
The cortical network was divided into four sub-networks (Figure 8.4, Table 8.2). The first and largest of these was formed by the temporal lobes and the orbitofrontal cortex. In this module the left pars triangularis, right frontal pole, and left fusiform gyrus had the highest participation indexes, whilst the bilateral entorhinal cortex, left middle temporal, and right pars orbitalis had the largest within-module degree Z-score. The second module was composed of the majority of the frontal and parietal lobes, with the right supramarginal gyrus, pars triangularis, and pars opercularis having the highest participation index and the left superior parietal the highest degree Z-score. Vision-related regions such as the occipital cortex and lingual gyrus formed a third, whilst the final network module was composed of the majority of the cingulate gyrus and the bilateral insulae. In the visionrelated module, the bilateral lateral occipital cortices had the highest participation index, whilst the left lingual gyrus had the largest degree Z-score. In the final module, the right posterior cingulate had the highest participation index and the right posterior cingulate the highest degree $\mathrm{Z}$-score. Those regions that have high participation indexes are those that interact most between modules whilst a high degree $\mathrm{Z}$ suggests that the region plays the most significant role within a particular network (Guimerà et al., 2007).

\subsection{Discussion}

Cortical $\mathrm{GABA}_{\mathrm{A}}$ receptor $\mathrm{BP}_{\mathrm{ND}}$ distributions were measured using FMZ-PET and intersubject correlations in regional $\mathrm{BP}_{\mathrm{ND}}$ used to create a connectivity graph. This graph identifies networks of brain regions where binding potentials vary across subjects in the same way. It was found that this receptor network shows small-world properties and that it can be subdivided into separate modules.

$\mathrm{GABA}_{A}$ receptors are dynamic entities. Their numbers and binding properties are altered by stimulus-related neural excitation (Arancibia-Carcamo and Kittler, 2009, Jacob et al., 2008, Jacobson-Pick and Richter-Levin, 2012, Jaffer et al., 2012, Liefaard et al., 2009) and by drug-induced changes in GABA concentrations (Frankle et al., 2012, Juhász et al., 2001, Prevett et al., 1995). Thus, in an individual, activity within any particular network will have effects on the receptors within that network. In this way variance across subjects that is coherent amongst different brain regions, such as is seen here, can arise. Patterns of receptors are necessarily constrained by anatomy, however, leading to their proposed role 

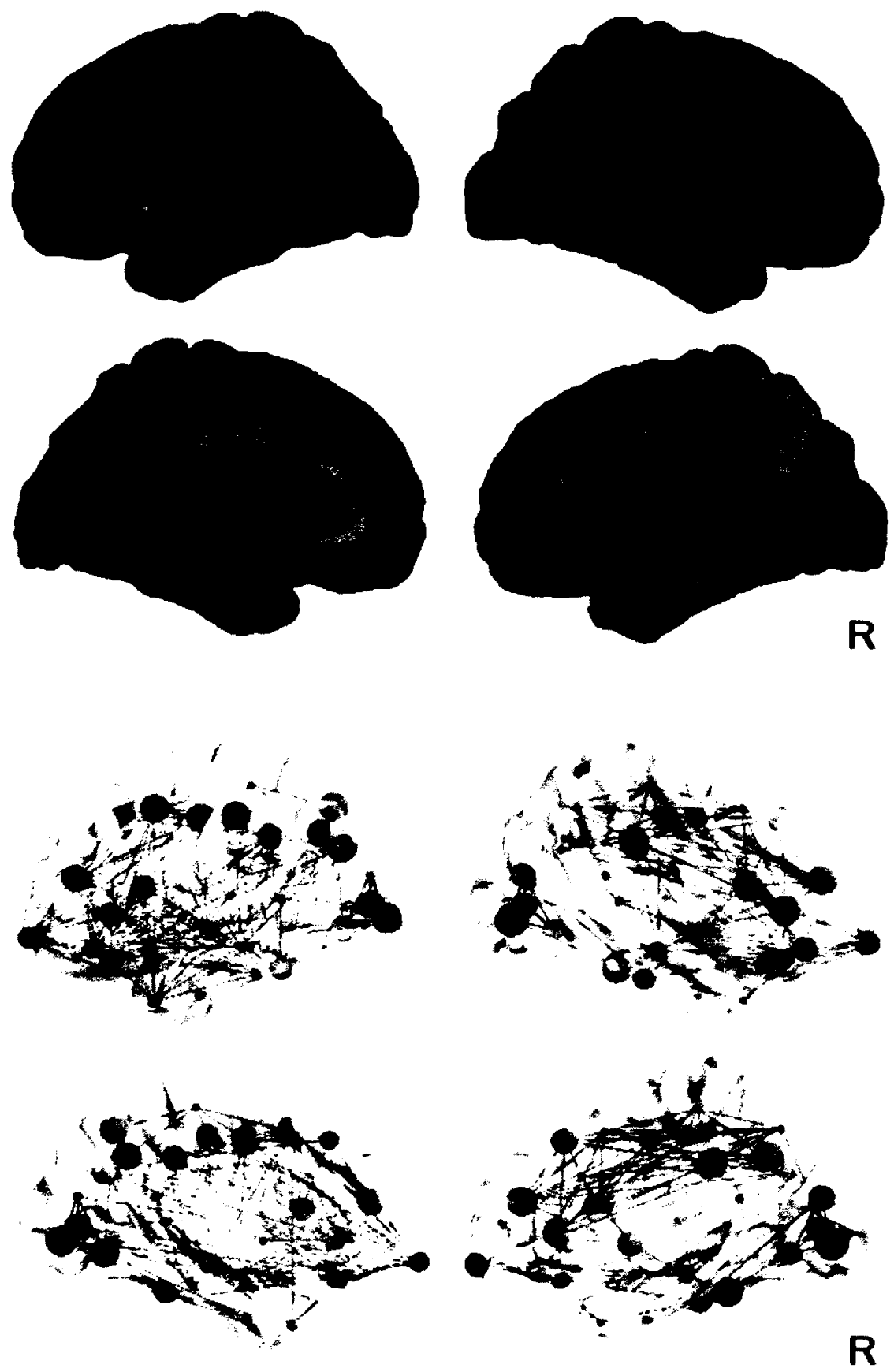

Figure 8.4: Network modules. Red - orbitofrontal-temporal, blue - vision-related, green - fronto-parietal, light blue - cingulo-insular. Sphere sizes indicate participation index magnitude. The modules identified fit well with known functional modules. 
as a bridge between the functional and anatomical domains. Support for this proposition comes from studies in rats, where, for example, stimulation of subcortical regions leads to altered $\mathrm{GABA}_{\mathrm{A}}$ receptor numbers in the cortex (Liefaard et al., 2009), as well as from studies of humans with epilepsy, where seizure-linked decreases in $\mathrm{GABA}_{\mathrm{A}}$ receptor numbers are seen in distributed regions of the brain (Juhász et al., 2009, Savic et al., 1996).

The small-world organisation observed is analogous to that seen with structural and functional brain networks (Achard et al., 2006, Bullmore and Bassett, 2011, Hagmann et al., 2008). Closer to the receptor level, such a network structure has also been identified at the synaptic level in the C. Elegans nervous system (Jarrell et al., 2012, Varshney et al., 2011 ) and at the cellular level in the human brainstem (Humphries et al., 2006). Betweenness centrality values identified cortical midline regions such as the anterior cingulate and precuneus as network hubs. This is also consistent with previous studies of functional and structural brain networks in humans, where the posterior midline in particular has been identified as a hub region (Hagmann et al., 2008). Those regions identified as hubs by their degree are also generally consistent with such studies, highlighting more frontal and temporal regions (Achard et al., 2006, He et al., 2007). These similarities suggest that the same fundamental structures are being measured at the the different levels of structure, function, and receptors.

Four sub-modules were found to exist within the receptor network. These are comparable to prior functional network studies in both number and general composition (AlexanderBloch et al., 2010, Meunier et al., 2010). Two modules were highly specific, consisting of vision-related regions around the occipital lobe and cortical midline regions plus the insula and parahippocampal gyrus, respectively. The other two could be described as frontoparietal and tempero-orbitofrontal and covered the majority of the cortex between them. Some lateralisation of these modules was evident, with the tempero-orbitofrontal module extending more dorsally in the left hemisphere (see Figure 8.4). This may be related to the handedness of the subjects, although this, whilst fitting with the left lateralisation of high degree regions seen, is speculative.

Focussing on the module composed of the cortical midline regions and insulae, the anterior midline regions and insulae have been identified as forming a network involved in multiple psychological contexts (Cauda et al., 2011, Craig, 2009, Seeley et al., 2007). Similarly, the anterior and posterior midline form the backbone of what is referred to as the default mode 


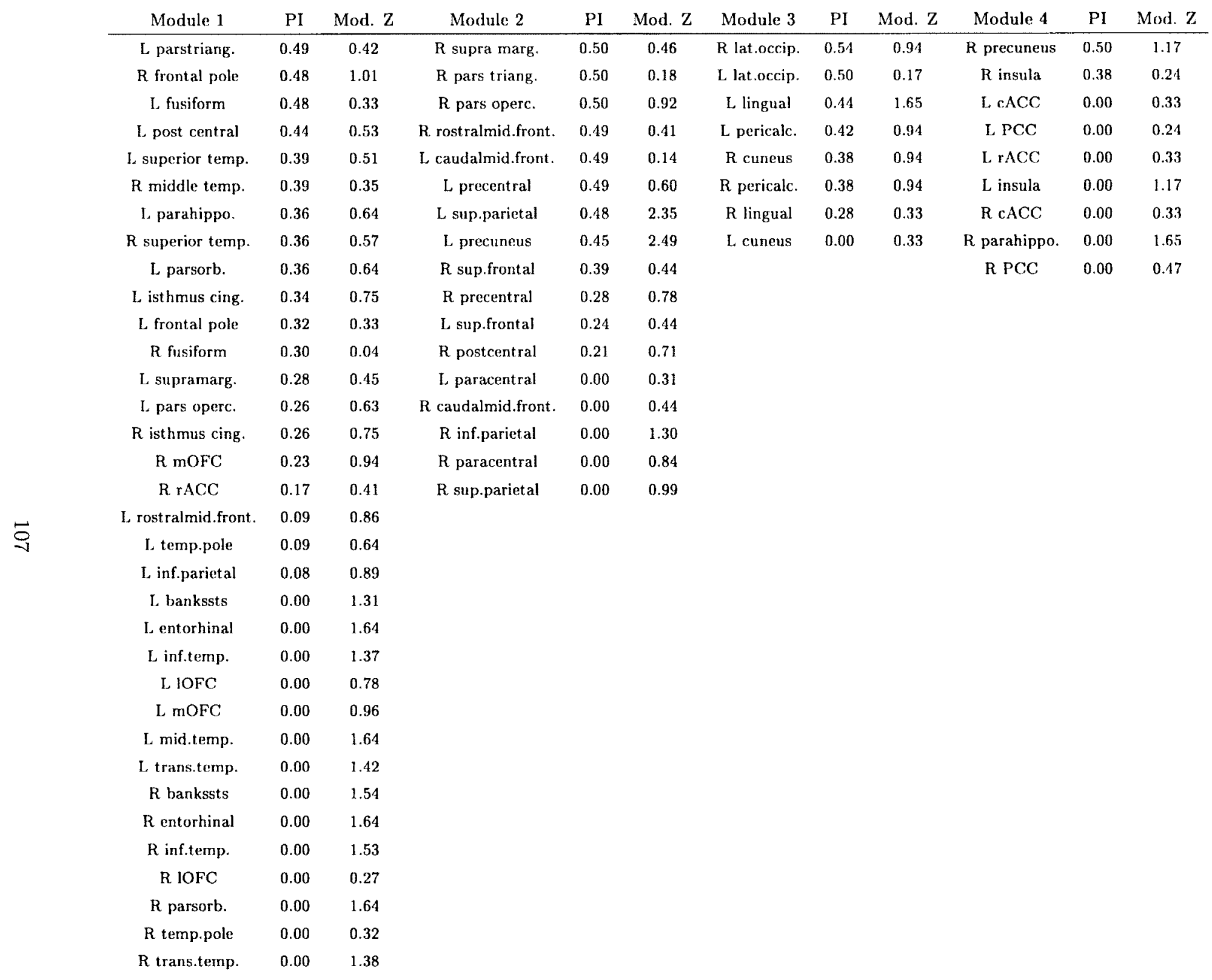

Table 8.2: Regions forming each of the four network sub-modules identified along with participation index (PI) and within-module degree z-score (Mod. Z) for each. 
network, which in turn shows a close interaction with the insula (particularly the right insula, which has a high participation index here - see Table 8.2)(Sridharan et al., 2008). These links between the regions composing this module thus provide indirect evidence that the receptor correlations seen are related to functional interrelations. This is bolstered by findings that show that task-induced activity within the cortical midline regions identified can be linked to inter-subject differences in $\mathrm{GABA}_{\mathrm{A}}$ receptors in humans (Wiebking et al., 2012).

To conclude, GABAA receptor networks in humans were shown to follow a small-world distribution and could be sub-divided into functionally meaningful modules. This provides a potential bridge between the functional and structural domain. Future studies may wish to investigate other receptor types or $\mathrm{GABA}_{\mathrm{A}}$ networks in disorders that are linked to changes in GABA-ergic function and functional or structural network properties, such as depression (Alcaro et al., 2010, Lord et al., 2012) or epilepsy (Fedi et al., 2006, Zhang et al., 2011).

\subsection{Acknowledgements}

Many thanks to A. Perna and K. Dedovic for their help with subject recruitment and to Dr. Z. Muñoz-Torres for her helpful comments. The study was supported by grants to G.N. from the Canadian Institutes of Health Research and Hope for Depression Research Foundation. 


\section{Chapter 9}

\section{Differential insula structural connectivity patterns predict BOLD responses to intero- and exteroception}

\subsection{Abstract}

The insula has been identified as an important region in intero- and exteroceptive awareness. Neuroimaging studies have additionally revealed a set of regions that co-activate with the insula in intero- and exteroceptive tasks, such as the supplementary motor area and thalamus. In addition, functional connectivity results have revealed patterns of correlated ongoing activity in many of the same regions. However, structural relationships between the insula and this network of regions during intero- and exteroception remain to be shown in humans. To provide such a link, we combined diffusion tensor imaging with a well established intero- and exteroception fMRI task. Combining these, models were trained to predict functional responses from structural connectivity. These models were shown to be effective and the regions of particular weight in them were taken to represent important structures in an intero- and exteroceptive network around the insula.

\subsection{Introduction}

The insula has been identified as being particularly involved in the processing of multimodal signals originating from both within and without the body (i.e., intero- and exteroceptive), with activation in this region reported in a large proportion of human neuroimaging studies (Chang et al., 2012, Sterzer and Kleinschmidt, 2010). Such functional imaging studies have also provided information as to the brain regions that along with the insula are concur- 
rently more active during intero- or exteroceptive processing. For example, interoceptive processing induced by heartbeat monitoring produces increased activity in, amongst other regions, the supplementary motor area (SMA) and thalamus (Critchley et al., 2004, Pollatos et al., 2007). Exteroceptive processing induced by attending to a visual stimulus, in contrast, is, in addition to the insula, associated with increased activity in, for example, the medial frontal gyrus and parietal cortex (Rees, 2007, Simmons et al., 2012). These patterns of co-activation suggest that the insula is an important locus within differentiable task-specific brain networks (Chang et al., 2012, Cauda et al., 2012, Craig, 2009).

This network role is supported by the anatomical connectivity of the insula, tracer and diffusion imaging studies having shown that it has interconnections with, for example, subcortical regions that directly receive bodily inputs (Craig, 2002), as well as with prefrontal regions that are implicated in 'higher-order' processes and with limbic regions thought to be involved in emotions (Cloutman et al., 2011, Mesulam and Mufson, 1982, Mufson and Mesulam, 1982, Ongür and Price, 2000). In addition, resting-state functional connectivity has been used to further elucidate the network role of the insula. Broadly in line with the structural connectivity, such studies have demonstrated correlational networks between the insula and limbic, prefrontal, and sensory-motor regions, amongst others (Deen et al., 2011, Farb et al., 2012, Simmons et al., 2012). However, although there is a strong indirect overlap between intero- and exteroceptive co-activation networks and the insula functional and anatomical networks that have been identified (Farb et al., 2012), no studies have directly investigated the relationship between task responses and insula network connectivity.

In order to bridge this gap, we sought to combine an intero- and exteroception fMRI task (Pollatos et al., 2007, Wiebking et al., 2010) with diffusion tensor imaging to investigate how anatomical connectivity to different brain regions impacts upon the functional responses seen within the insula. Anatomical connection probabilities from each voxel within the insula to all other regions in the brain were used to create a model that predicted the voxelwise functional response within the insula (Saygin et al., 2012). The model properties then give information as to which brain regions contribute most to the insula response to interoor exteroception. It was hypothesised that intero- and exteroceptive responses would be predicted by the connectivity from dissociable regions, and that these would reflect to a large degree the co-activation and functional connectivity patterns that have been reported previously (Farb et al., 2012, Wiebking et al., 2010). 


\subsection{Methods}

\subsubsection{Participants}

Twenty-six healthy participants were scanned using a Siemens 3 Tesla Trio magnet. The data from six participants were rejected due to excessive head movement during the functional task, leaving twenty participants included in the analysis ( 7 females; mean age $=$ 23.1 years, range: 19-32 years). Participants were screened for psychiatric or neurological disorders, recreational drug use, and depression, the latter using the Beck Depression Inventory-II with a cut-off score of four (Beck et al., 1996). All participants gave their written informed consent and were compensated financially for their participation. Approval for the study was obtained from the ethics committee at McGill University. A separate analysis of functional task is reported elsewhere (Wiebking et al., 2012).

\subsection{2 fMRI}

Functional EPI scans were acquired using a body coil transmit and 32-channel receive headcoil. Forty-seven slices aligned at $-30^{\circ}$ from the AC-PC plane and covering the whole brain were acquired per volume, with a total of 580 volumes being acquired (1316 s) over the task run $\left(\mathrm{FoV}=205 \times 205 \mathrm{~mm}^{2}\right.$; spatial resolution $=3.2 \times 3.2 \times 3.2 \mathrm{~mm}^{3} ; \mathrm{T}_{\mathrm{E}}=25 \mathrm{~ms}$; $\mathrm{T}_{\mathrm{R}}=2270 \mathrm{~ms} ;$ flip angle $\left.=90^{\circ}\right)$. The first five volumes were discarded to exclude saturation effects. A high-resolution $\mathrm{T}_{1}$-weighted anatomical image was also acquired (MPRAGE; FoV $=205 \times 205 \mathrm{~mm}^{2}$; spatial resolution $=1 \times 1 \times 1 \mathrm{~mm}^{3} ; \mathrm{T}_{\mathrm{E}}=3.02 \mathrm{~ms} ; \mathrm{T}_{\mathrm{R}}=2000 \mathrm{~ms}$; flip angle $=5^{\circ}$ ) for alignment purposes and the creation of subject-specific ROIs.

Participants took part in a well established intero/exteroception task (Critchley et al., 2004, Pollatos et al., 2007, Wiebking et al., 2010). This consisted of three separate conditions: an internally directed perception period (interoception), an externally directed perception period (exteroception), and fixation periods. Conditions lasted 6-10 seconds and were presented in a pseudo-random order. During the interoception conditions, subjects counted their own heartbeat. Analogously, during the exteroception condition subjects counted instances of a tone played through headphones attached to the scanner. Subjects reported the number of counted heartbeats or tones after each trial using a visual analogue scale (3.5 
s). In order to make the difficulty of both tasks comparable, tones were presented at an individually determined volume that was just noticeable, like the heartbeat. The general presentation frequency of the tones was adapted to correspond to each subject's pulse-rate. In order to reduce habituation effects, the onset time of the tones was jittered by $200 \mathrm{~ms}$ from this general frequency. During the fixation condition, subjects were instructed to relax and minimise any mental effort.

The processing of all MRI data was carried out with the FSL suite of tools (Smith et al., 2004, Woolrich et al., 2009). Functional images were slice-time corrected, realigned to the middle volume of the run, high-pass filtered $(90 \mathrm{~s})$, and smoothed with a $6 \mathrm{~mm}$ at FWHM Gaussian kernel. White matter and cerebro-spinal fluid (CSF) masks were created from segmented anatomical images (produced using FAST), thresholded for each tissue type at a probability of 0.95 , made into binary masks, and then eroded by two voxels to ensure no overlap with grey matter voxels. The masks were then aligned to the functional images and the average timecourse from each tissue extracted. These timecourses were included in the functional analysis model, along with the six parameters from the realignment step, in order to minimise physiological noise and head-movement effects. Using the FEAT5 tool, functional data were entered into a general linear model analysis (Friston et al., 1995). The model used consisted of each condition's timings convolved with a double-gamma haemodynamic response function, plus the eight confound regressors. Contrasts of [int $>$ ext], [int $>$ fix], and [ext $>$ fix] were calculated, producing a set of subject t-statistic maps. These maps were then aligned with individual subject's DTI images. Finally, the t-statistic maps were standardised for each seed region by dividing each voxel's value by the mean across voxels within the ROI and then dividing by the standard deviation.

\subsubsection{Regions of interest}

$\mathrm{T}_{1}$-weighted anatomical images were segmented into individually defined cortical and subcortical regions of interest (ROIs) using Freesurfer (Desikan et al., 2006, Fischl et al., 2002, 2004)(http://surfer.nmr.mgh.harvard.edu/). The parcellated brain maps, consisting of a total of 86 regions, were then registered to each subject's DTI image to allow them to be used as seed (left or right insula) and target regions (all other 85 grey matter regions) for the DTI analysis. A single region was constructed out of the multiple ventricle parcellations (i.e., $1^{\text {st }}$ to $4^{\text {th }}$ ventricle) for use as an exclusion mask in the DTI analysis. 


\subsubsection{DTI}

Diffusion-weighted images were acquired using a body coil transmit and a 32-channel receive headcoil. Sixty-four slices were aligned parallel to the AC-PC axis and data acquired for 99 diffusion weighting directions with a resolution of $1.9 \times 1.9 \times 1.9 \mathrm{~mm} 3$ ( FoV $=243 \times$ $243 \mathrm{~mm} 2$, matrix $=128 \times 128$, slice thickness $=1.9 \mathrm{~mm}, \mathrm{~T}_{\mathrm{E}}=89 \mathrm{~ms}, \mathrm{~T}_{\mathrm{R}}=8300 \mathrm{~ms}$, Fourier factor $=6 / 8,99$ acquisitions with $\mathrm{b}=1000 \mathrm{~s} / \mathrm{mm}^{2}, 10$ acquisitions with $\mathrm{b}=0$ ). In addition, a field map was acquired to correct for field distortions (FoV $=256 \times 256 \mathrm{~mm} 2$, matrix $=128 \times 128$, slice thickness $=2 \mathrm{~mm}, \mathrm{~T}_{\mathrm{E} 1}=5.09 \mathrm{~ms}, \mathrm{~T}_{\mathrm{E} 2}=7.55 \mathrm{~ms}, \mathrm{~T}_{\mathrm{R}}=1000$ $\mathrm{ms}$, Fourier factor $=6 / 8$ ).

Raw data were corrected for field distortions, eddy current distortions, and motion artifacts using FSL's FUGUE and eddy_correct tools. DTIFIT was then used to fit a diffusion tensor model at each voxel. A connectivity distribution model was calculated from each seed region voxel to each of the target ROIs using the probtrackx tool (25,000 streamlines). As described above, a ventricle exclusion mask was used to disregard tracks passing through the CSF.

\subsubsection{Model construction}

The construction of the predictive models followed the methods described by Saygin and colleagues (2012). For an overview, see Figure 9.1. Briefly, the DTI analysis resulted in an $\mathrm{N} \times \mathrm{M}$ matrix, $\boldsymbol{A}$, for each subject, where $\mathrm{N}$ is equal to the number of voxels within the seed region and $M$ was equal to the number of target ROIs (85). Each point in the matrix thus represents a connectivity weight from an individual seed voxel to a particular target ROI. The fMRI analysis resulted in an $\mathrm{N} \times 1$ matrix, $\boldsymbol{B}$, per subject, where $\mathrm{N}$ was again equal to the number of voxels within the seed region and each value was a standardised t-statistic for the particular contrast.

Adopting a leave-one-subject-out cross validation approach (LOOCV), least squares models were iteratively trained on the vertically concatenated $\boldsymbol{A}$ and $\boldsymbol{B}$ matrices for all but one subject. This model was then applied to the remaining subject's matrix, $\boldsymbol{A}$, to predict standardised t-statistics for each voxel (i.e., a simulated $\boldsymbol{B}$ matrix). The simulated and 
actual t-statistics were compared to give voxel-wise errors, which were then averaged to give a mean absolute error (MAE) for each subject, a lower MAE meaning a more accurate model.

To test the efficacy of the structural connectivity model, it was compared to a number of other approaches (all, again, using an LOOCV approach). Comparisons were made using appropriate Student's t-tests. Firstly, structural matrices were randomly permuted by voxel (5000 permutations) to give a distribution of random MAEs to which the actual MAE was compared. Secondly, matrices representing the Euclidean distance between each seed voxel and each of the target ROIs were used instead of the structural connectivity matrices and the resulting voxel-wise errors compared for each subject. Finally, the group fMRI results were used in a LOOCV fashion, comparing the standardised voxel-wise group results iteratively to the actual results of the remaining subject. This latter step was carried out in MNI space, all other analyses having been carried out in native DTI space. A significance threshold of $\mathrm{p}<0.001$ was used for comparisons between approaches (Saygin et al., 2012).

Model coefficients were used to identify potential differences between conditions in regional contributions to functional responses. The [int $>$ fix] and [ext $>$ fix] contrasts were focused upon as these individual contrasts provide information as to those region contributing to the intero- and exteroceptive processes themselves, rather than to the difference between them (i.e, the [int > ext] contrast). Mean coefficients were converted to z-scores - those with an absolute $z>1.3$ (i.e., the $90^{\text {th }}$ percentile) were deemed of interest for reporting and discussion.

As prior work has shown a distinction between the anterior and posterior insula in the context of intero- and exteroception (Craig, 2002, Farb et al., 2012), the calculated connectivity matrices of the insula seed regions were additionally divided into anterior and posterior regions by dividing the individual insula ROIs on the coronal plane at the approximate midpoint of the insula, as determined in MNI space (left division: -33 17 -1; right division $3717-4$ ). This division reflects a division of the insula into an anterior part, a posterior part, and a shared transition region between them, as described in previous studies (Cauda et al., 2011, Farb et al., 2012, Mesulam and Mufson, 1982). Additional models were then calculated using these connectivity matrices to give coefficients specific to the anterior and posterior insulae. A more fine-grained division of the insula was not employed in order to retain a large enough number of voxels for the training of each model. 

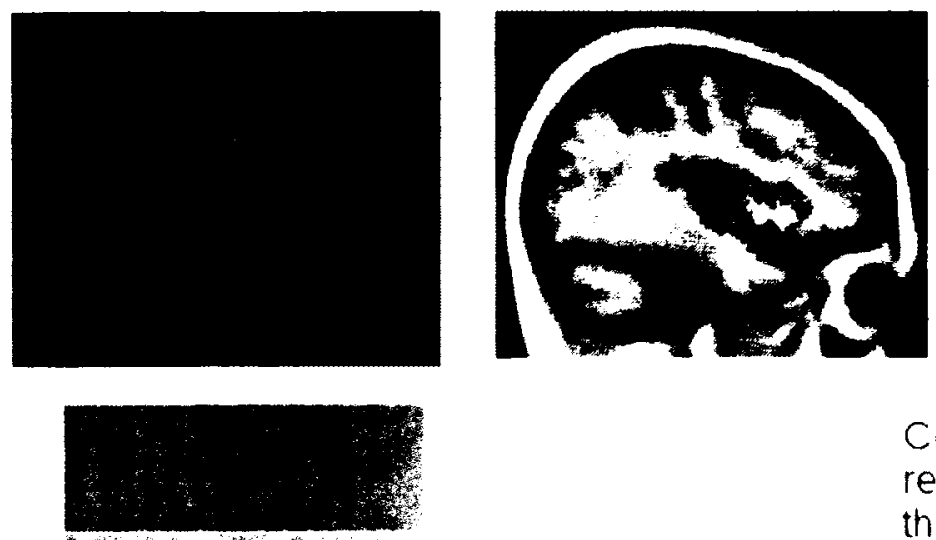

से का?

A

B

Connectivity and functional responses for each vorel within the insula for all subjects but one merged into matrices. $\boldsymbol{A}$ and $\boldsymbol{B}$.

Least squares model then trained on matrices.

i.e., $B=m A+e r r$

Fiesulting set of coefficients. $\boldsymbol{m}$. then applied to remaining subject's connectivity' matrix.

$m$
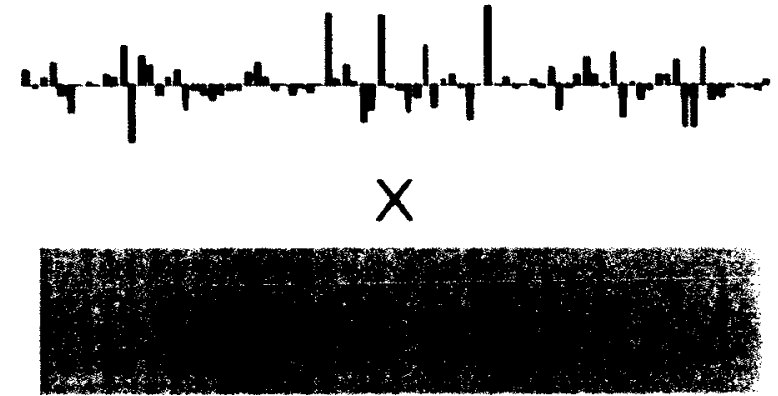

Gives predicted functional

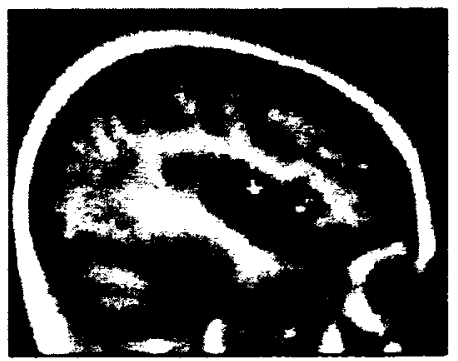
responses. Compared to real responses to give MAE.

Repeated for all subjects.

Figure 9.1: Overview of the analysis approach used. 


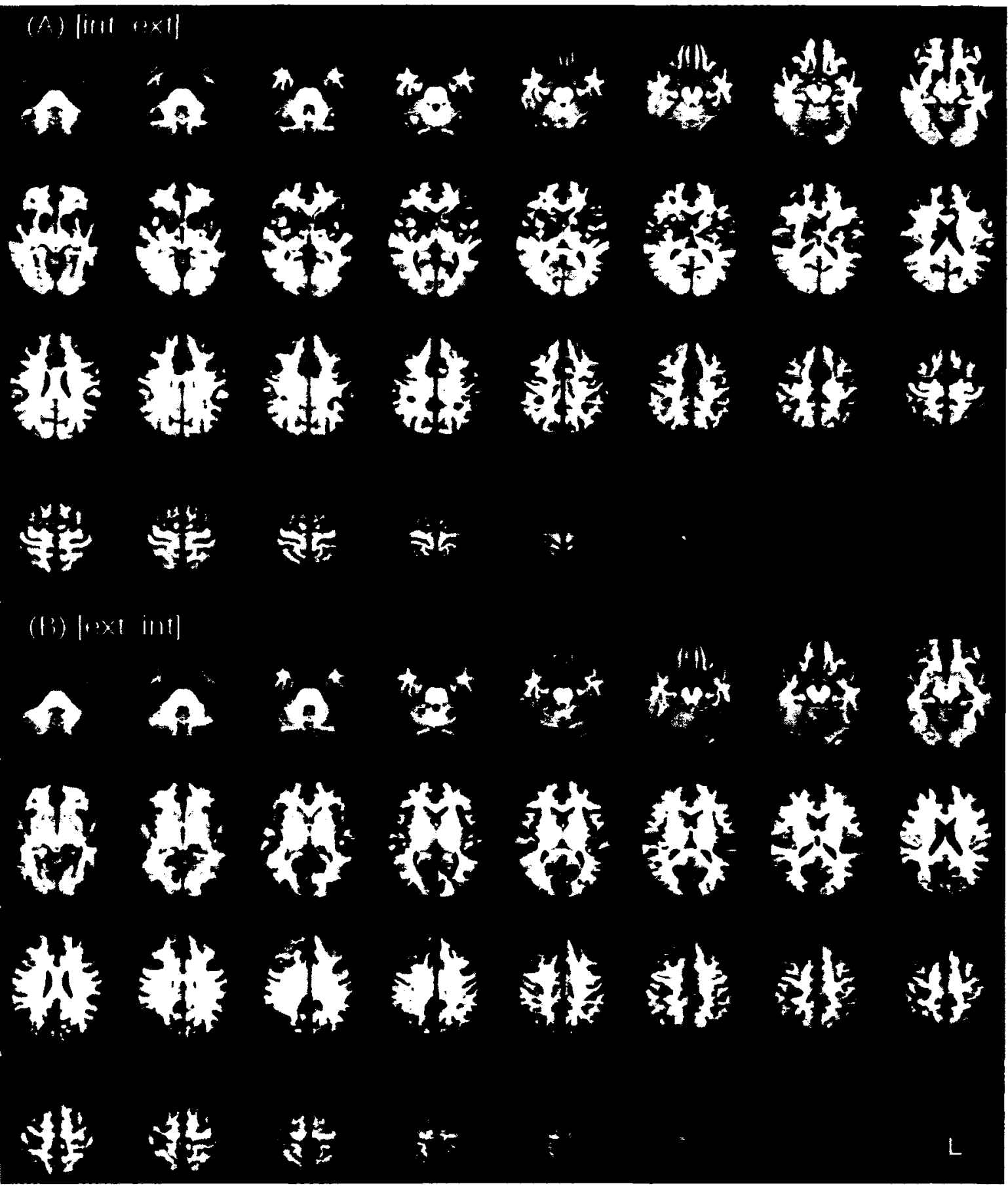

Figure 9.2: Group image for (A) contrast [int $\cdots$ ext] and (B) contrast [ext $\cdots$ int]. Results displayed on group mean anatomical image $\left(\mathrm{p}_{\mathrm{FWE}} \cdots 0.05\right)$. 


\subsection{Results}

The intero/exteroception task produced results that are consistent with prior studies (Pollatos et al., 2007, Wiebking et al., 2010). Increased activity was seen in the bilateral anterior insula in the contrast [int $>\mathrm{ext}$ ], along with motor areas, and the ventral striatum, amongst others (Figure 9.2A, Supplementary table $2 \mathrm{~A}$ ). The contrast [ext $>$ int] revealed areas of increased activity in, for example, the auditory and visual cortices, the $\mathrm{mPFC}$, and the precuneus (Figure 9.2B, Supplementary table 2B). Regions of increased activity consistent with these results were seen in the $[$ int $>$ fix] and $[$ ext $>$ fix] contrasts (Supplementary figure $1 \mathrm{C} \& D$, Supplementary table 2C\&D).

The structural model predicted functional responses (Figure 9.3) with a mean MAE across regions and conditions of $0.74 \pm 0.08$. This means that, on average, estimated t-statistics were 0.74 less or more than each subject's true normalised t-statistic. When compared to the standard fMRI analysis (mean MAE $=0.70 \pm 0.13$ ), the structural model predicted functional responses with equivalent accuracy for all regions and in all conditions other than [int > fix], where the fMRI model performed better (Table 9.1). The structural model performed better than random permutations and the Euclidean distance model in all regions and all conditions. The MAE values obtained were comparable to previous studies (Saygin et al, 2012).

Regions identified in the standard GLM results from the current dataset (Figure 9.2, Supplementary figure 1 and tables 1-4) and in prior studies of intero- and exteroception activity (Critchley et al., 2004, Pollatos et al., 2007, Simmons et al., 2012) were shown to have large influences in the structural models. For example, in addition to regions close to the the insula such as the operculum and pars triangularis, the precentral gyrus was found to have a high positive coefficient for the [int > ext] model for both the left and right insula, as did the middle frontal gyrus. In contrast, the precuneus and rostral ACC had large negative coefficients for the same model, equivalent to large positive coefficients in the inverse [ext $>$ int] contrast (see Figure 9.4 and Table 9.2).

Clear distinctions could be seen in model coefficients between the two conditions (intero- and exteroception) and four regions (left and right insula, anterior and posterior). Full results for the individual [int $>$ fix] and [ext $>$ fix] can be found in Figure 9.5 and Supplementary 


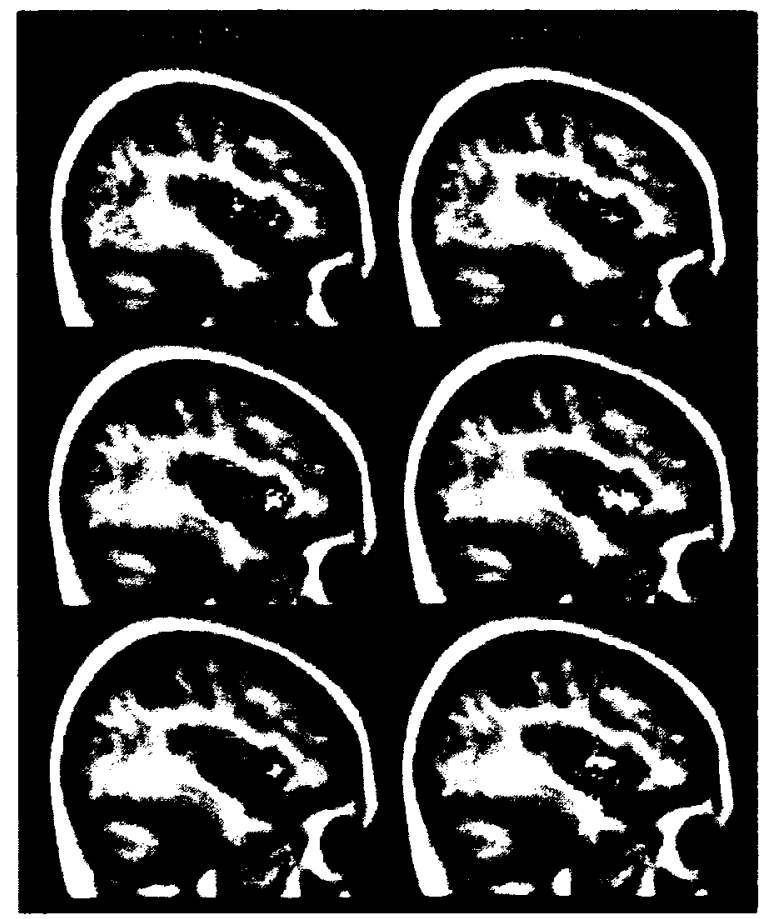

Figure 9.3: Predicted and actual right insula [int ext] functional responses for three participants. Modelled results can be seen to match well with actual values. $x$ : 38. shown on group mean anatomical image in MNI space. 


\begin{tabular}{|c|c|c|c|c|}
\hline Contrast & Structural & Group fMRI & Permutation & Distance \\
\hline \multicolumn{5}{|c|}{ Left insula } \\
\hline ext] & $0.78 \pm 0.12$ & $0.77 \pm 0.17$ & $0.81 \pm 0.03^{*}$ & $4.90 \pm 6.07^{*}$ \\
\hline $\mathrm{fix}]$ & $0.61 \pm 0.0 \bar{\tau}$ & $0.53 \pm 0.10 \dagger$ & $0.81 \pm 0.03^{*}$ & $3.33 \pm 4.28^{*}$ \\
\hline$[e x t$ & $0.73 \pm 0.14$ & $0.74 \pm 0.21$ & $0.79 \pm 0.04^{*}$ & $4.58 \pm 7.68^{*}$ \\
\hline \multicolumn{5}{|c|}{ Right insula } \\
\hline ext] & $0.75 \pm 0.09$ & $0.73 \pm 0.1 \tilde{\jmath}$ & $0.82 \pm 0.03^{*}$ & $2.86 \pm 2.38^{*}$ \\
\hline [int & $0.59 \pm 0.08$ & $0.48 \pm 0.11 \uparrow$ & $0.82 \pm 0.03^{*}$ & $2.82 \pm 3.19^{*}$ \\
\hline [ext & $0.68 \pm 0.13$ & $0.66 \pm 0.23$ & $0.80 \pm 0.04^{*}$ & $4.39 \pm 4.57^{*}$ \\
\hline
\end{tabular}

Table 9.1: Mean average errors (MAE) for left and right insula models. MAEs are given for the structural connectivity model, the group fMRI results, random permutations of structural comnectivity; and the Euclidean distance model. * indicates that these models performed less well than the structural model in predicting functional responses $(p, 0.001)$. $\dagger$ indicates models that performed better than the structural model $(p-0.001)$. Unmarked values are not significantly different from the structural model.

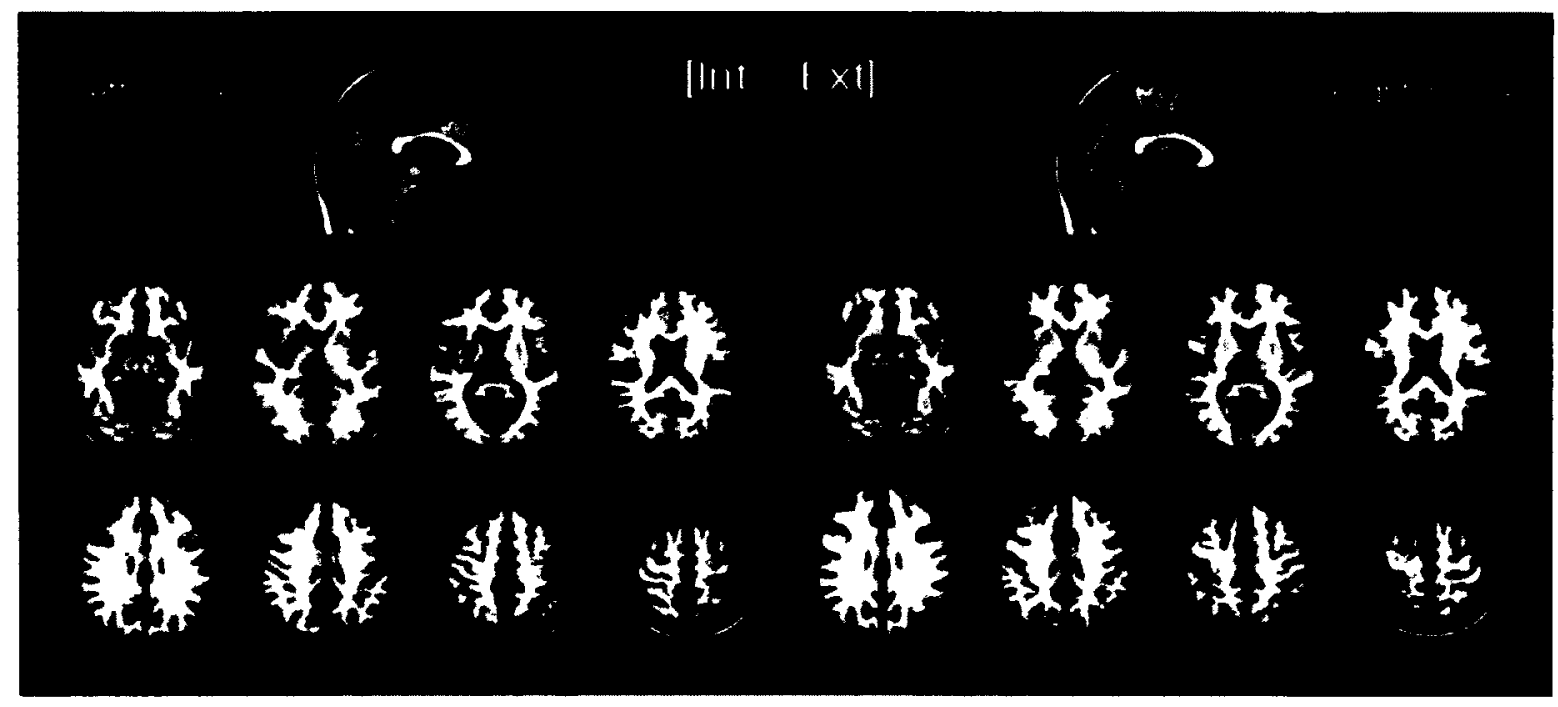

Figure 9.4: Model coefficients for [int ext] contrast in left and right insula. Warmer and colder colours represent larger positive and negative weights, respectively. 


\begin{tabular}{cccccccc} 
Region & \multicolumn{2}{c}{ Coefficient (C.I.) } & Region & \multicolumn{3}{c}{ Coefficient (C.I.) } \\
\hline & Left insula & & \multicolumn{4}{c}{ Right insula } \\
R cACC & 0.23 & $(0.18$ & $0.30)$ & R precentral & 0.23 & $(0.21$ & $0.24)$ \\
L pars.operc. & 0.21 & $(0.17$ & $0.23)$ & R pars.operc. & 0.17 & $(0.16$ & $0.18)$ \\
L precentral & 0.20 & $(0.19$ & $0.23)$ & R thalamus & 0.13 & $(0.10$ & $0.16)$ \\
R caudate & -0.18 & $(-0.24$ & $-0.14)$ & R bankssts & 0.12 & $(0.09$ & $0.12)$ \\
R precuneus & -0.13 & $(-0.16$ & $-0.08)$ & L inf.parietal & 0.12 & $(0.09$ & $0.14)$ \\
R rACC & -0.11 & $(-0.16$ & $-0.10)$ & brainstem & -0.10 & $(-0.13$ & $-0.06)$ \\
R thalamus & 0.11 & $(0.10$ & $0.12)$ & R sup.parietal & 0.10 & $(0.07$ & $0.13)$ \\
L sup.parietal & 0.11 & $(0.09$ & $0.17)$ & R sup.frontal & -0.10 & $(-0.12$ & $-0.08)$ \\
L postcentral & -0.11 & $(-0.13$ & $-0.09)$ & R sup.temp. & -0.10 & $(-0.11$ & $-0.09)$ \\
L trans.temp. & -0.11 & $(-0.12$ & $-0.10)$ & L pars.triang. & 0.09 & $(0.07$ & $0.11)$ \\
R rMFG & 0.11 & $(0.07$ & $0.16)$ & R peri.calc. & -0.09 & $(-0.10$ & $-0.07)$ \\
R putamen & 0.10 & $(0.06$ & $0.15)$ & R pars.triang. & 0.08 & $(0.07$ & $0.11)$ \\
R paracentral & 0.09 & $(0.07$ & $0.12)$ & R hippo. & -0.08 & $(-0.10$ & $-0.07)$ \\
R pars.operc. & -0.09 & $(-0.11$ & $-0.08)$ & R lOFC & -0.08 & $(-0.11$ & $-0.06)$ \\
brainstem & -0.09 & $(-0.12$ & $-0.07)$ & R NAc & 0.08 & $(0.07$ & $0.10)$ \\
L rMFG & -0.08 & $(-0.10$ & $-0.03)$ & R postcentral & -0.08 & $(-0.09$ & $-0.07)$ \\
R mOFC & 0.08 & $(0.06$ & $0.11)$ & R cACC & 0.07 & $(0.05$ & $0.11)$ \\
L PCC & -0.08 & $(-0.10$ & $-0.06)$ & R. lingual & 0.07 & $(0.05$ & $0.09)$ \\
R lat.occ. & -0.08 & $(-0.09$ & $-0.07)$ & R rACC & -0.06 & $(-0.09$ & $-0.06)$ \\
L sup.temp. & -0.07 & $(-0.09$ & $-0.06)$ & L sup.temp. & -0.06 & $(-0.08$ & $-0.05)$ \\
& & & & R isthmusCC & 0.06 & $(0.05$ & $0.10)$ \\
& & & & R lat.occ. & -0.06 & $(-0.07$ & $-0.05)$ \\
& & & & R precuneus & -0.06 & $(-0.10$ & $-0.03)$
\end{tabular}

Table 9.2: Coefficients and 95\% confidence intervals for the model predicting [int $>$ ext] functional responses in the left and right insula. 
tables D. 3 \& D.4. Perhaps most noticeable amongst the results was a right posterior focus of interoceptive input regions. For example, only the right posterior insula showed positive thalamic coefficients or large coefficients for the brainstem (although these were negative). In contrast, the models for activity in the left posterior insula were more influenced by connectivity to regions of, for instance, the posterior cingulate and sensory-motor regions (Figure 9.5 and Supplementary table D.5). Less lateralisation was seen for exteroception, with auditory regions around the left or right superior temporal cortex influencing their respective insulae.

\subsection{Discussion}

Subject BOLD responses within the insula to an intero- and exteroception task were predicted from the anatomical connectivity profile of the region. The overall accuracy of this prediction equalled that of a standard fMRI group analysis and was superior to control models. The regions that had the highest weighting in each of the predictive models were consistent with previous studies of task activation.

The effectiveness of the structural model, being equal to a standard GLM group analysis in all cases apart from the [int $>\mathrm{fix}$ ] contrast, provides evidence for the close link between structural networks in the brain and responses to specific conditions (Catani et al., 2012). Similarly, the overlap in the regions that had large weightings in the model and regions that are identified in standard GLM intero- and exteroception studies (Critchley et al., 2004, Pollatos et al., 2007), such as the precentral gyrus or precuneus, provides evidence for a potential causative interaction between these regions, beyond purely correlative co-activity (Saygin et al., 2012).

In the case of interoception, the right posterior insula model had a specifically high weighting for the thalamus (with this region not having a high positive weight for any other region or condition). This is consistent with the interoceptive pathway described by Craig (Craig, 2002), which is lateralised to the right and consists of the brainstem connecting to the thalamus and then onwards to the right posterior insula. From the right posterior insula it is then thought that interoceptive signals are transferred onwards to the right and left 


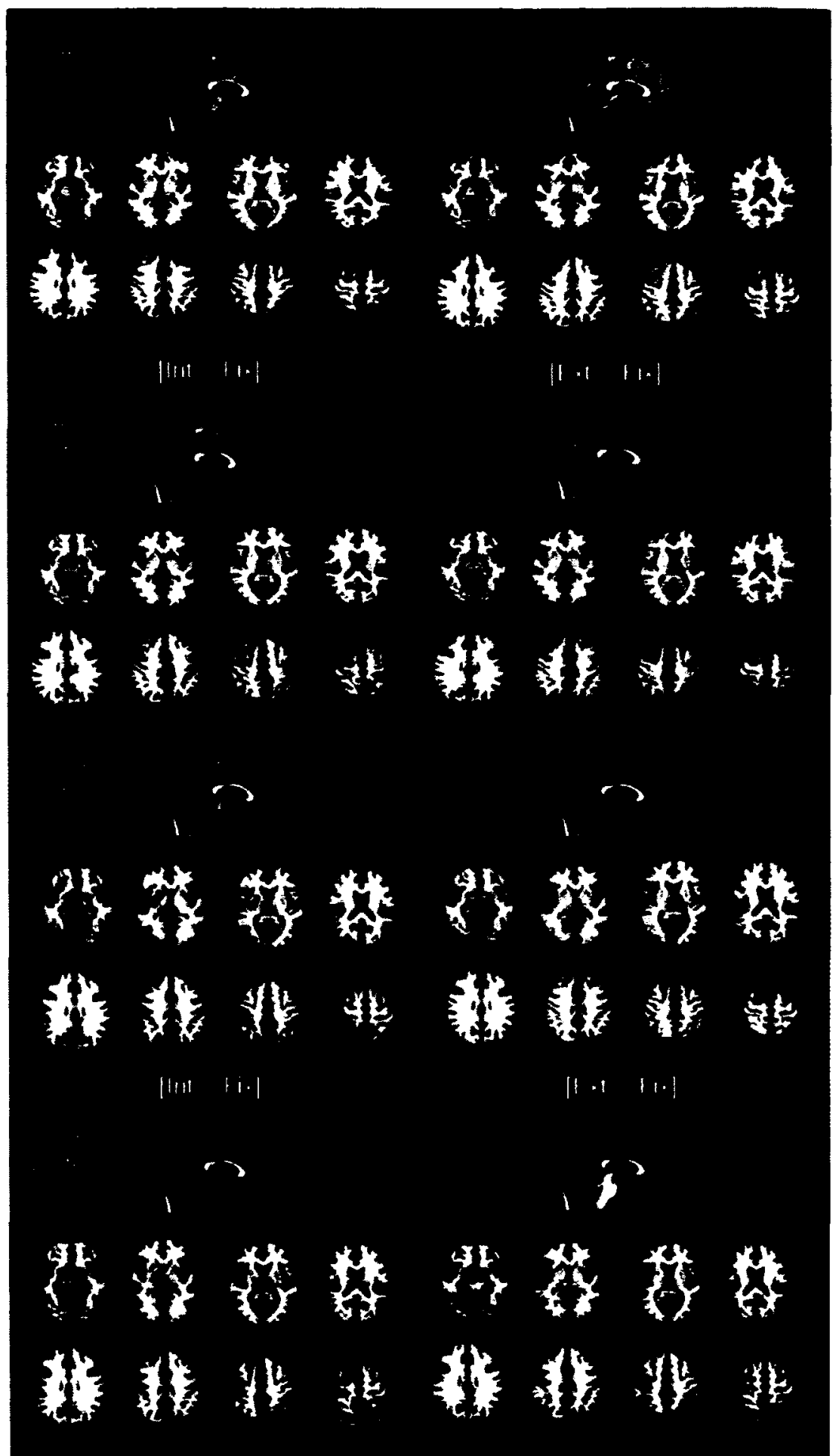

Figure 9.5: Model coefficients for left and right anterior and posterior insula for [int - fix] and $[$ ext fix] contrasts. Differential patterns can be seen between the left and right insula for both contrasts. Warmer and colder colours represent larger positive and negative weights, respectively. 
anterior insulae. This feeding forward from the right posterior insula is consistent with the pattern of regional weights seen in the left anterior insula in the [int > ext] contrast, where the $\mathrm{ACC}$, which may here act as a communicating region between the left and right insulae (Craig, 2009, Simmons et al., 2012), has a high positive weighting.

As well as conforming with previous intero- and exteroception task results, the regions identified as having task-relative structural connectivity with the insula overlapped well with regions identified in insula functional connectivity studies. For example, the high weightings of the the mid-cingulate cortex and superior frontal lobe (including the SMA) in the [int $>\mathrm{fix}$ ] contrast for the posterior insula is mirrored by a similar resting functional connectivity pattern (Cauda et al., 2011, Deen et al., 2011) and task-specific functional connectivity patterns (Simmons et al., 2012). Similarly, weightings of, for example, the ventral striatum or precuneus are in line with resting functional connectivity between the insula and these regions (Cauda et al., 2011, Deen et al., 2011).

Notable is that a wide range of regions contribute to the predictive model, suggesting that complex distributed networks are involved in the intero- and exteroceptive task employed, in line with the suggested integrative role of the insula (Critchley, 2005, Kimura et al., 2010, Sterzer and Kleinschmidt, 2010). Similarly, the pronounced involvement of the anterior cingulate in the models can be seen in light of the consistent co-activation of these regions across a wide range of tasks (Craig, 2009). This relationship between the regions has led to suggestions that they play a central role in contextualising stimuli (Cauda et al., 2012, Northoff, 2012, Roy et al., 2012, Seeley et al., 2007). The extended and often overlapping networks feeding into the insula that are seen here, coupled with the importance of cingulate connectivity in the predictive models, would fit with such a multi-domain role.

Disruptions in insular intero- and exteroceptive processing as been implicated in major depressive disorder (Nagai et al., 2007, Wiebking et al., 2010). At the same time, distributed alterations in grey (Konarski et al., 2008, Liu et al., 2012) and white (Liao et al., 2012) matter structures are seen in this condition, along with alterations in functional network properties (Lord et al., 2012, Northoff et al., 2011). An interesting next step would thus be to apply the method adopted here to a patient population, as it provides a potential bridge between these different domains, giving a link between structural and functional networks and task responses. 
A number of limitations of the current study must be considered. Firstly, the brainstem and thalamus ROIs did not differentiate between the constituent structures within these regions. This may explain the higher weighting of the brainstem in the anterior insula during exteroception than the posterior insula during interoception, as would perhaps be expected given the path that interoceptive signals take from the body (Craig, 2002). An effective segmentation of these regions is thus required, although this may be technically challenging. Secondly, the model was not tested on an independent group. Although the LOOCV approach helps to ameliorate over fitting of the model, this cannot be ruled out. Repetition with another sample is thus required. Finally, the paradigm used involves only one form of interoceptive awareness - heartbeat counting. Although studies using different paradigms show very similar functional results (Chang et al., 2012, Farb et al., 2012), comparing the networks seen here with those related to different interoceptive processes may be informative.

To conclude, a combination of structural and functional imaging was employed to identify brain networks that are involved in intero- and exteroception. The structural networks identified were found to overlap well with previously identified co-activating regions and functional connectivity networks. The results provide a potential linkage between the functional and structural domain in the context of intero- and exteroception.

\subsection{Acknowledgements}

Many thanks to A. Perna and K. Dedovic for their help with recruiting subjects, O. Lyttleton for his technical input, and the staff at the MNI for their skillful assistance. The work was supported by grants to G.N. from the Canadian Institutes of Health Research and the Michael Smith Foundation. 


\section{Chapter 10}

\section{Reported early-life stress predicts mPFC resting-state glutamate and entropy with concomitant effects on aversion-related brain responses in healthy adults}

\subsection{Abstract}

Traumatic aversive experiences in childhood have been linked to mental health problems in adulthood. Research in human and non-human animals has shown that early-life stress (ELS) has effects upon neural activity in the resting-state, on the glutamatergic system, and on brain responses to aversive stimuli. These effects can be linked to psychiatric disorders - such as post-traumatic stress disorder, depression, and anxiety disorders - that are greatly influenced by ELS. To investigate the effect of ELS on these systems, restingstate fMRI, aversion task FMRI, and glutamate MRS were combined with the Childhood Trauma Questionnaire (CTQ) in a group of non-clinical subjects. It was found that CTQ scores correlated negatively with resting-state glutamate levels in the medial prefrontal cortex (mPFC), and positively with resting-state activity entropy in the same region. CTQ scores, $\mathrm{mPFC}$ glutamate, and $\mathrm{mPFC}$ entropy were found to correlate with neural responses to the anticipation of aversion in regions within the aversion network. In particular, an overlap between all measures was seen in the sensory-motor cortex and left insula. Taken together, the results highlight the impact that ELS has on multiple inter-related brain

systems, even in healthy subjects with no history of psychiatric illness, and provide insight into the mechanisms underlying ELS effects on brain function in humans. 


\subsection{Introduction}

Traumatic experiences in childhood are sadly prevalent in society and have been linked to mental health problems in adulthood (Edwards et al., 2003, Kuo et al., 2011, Nelson et al., 2002, Turecki et al., 2012). A growing body of studies indicates that early-life stress (ELS) leads to alterations in brain structure and function, which also manifest at the cognitive level (Bellis and Hooper, 2009, Gould et al., 2012, Majer et al., 2010, Pluck et al., 2011), in both clinical and non-clinical populations (Bremner et al., 2003, Grant et al., 2011, Hart and Rubia, 2012, Sheu et al., 2010, Teicher et al., 2012, Thomaes et al., 2012). In addition to altered functional brain responses to tasks (Grant et al., 2011, Mueller et al., 2010, Thomaes et al., 2012), the properties of the resting brain (also described as intrinsic activity) have been shown to be affected by ELS (Bluhm et al., 2009, McFarlane et al., 2005). These resting-state changes include structural and functional alterations to brain networks, such as the default-mode network (DMN) (Bellis et al., 2002, Bluhm et al., 2009, Jackowski et al., 2008, Tomoda et al., 2009), that develop over childhood (Supekar et al., 2010, Uddin et al., 2011). Based on dysfunctions in the DMN in a range of mental health problems associated with ELS, including post-traumatic stress disorder (PTSD), major depressive disorder (MDD), and anxiety disorders, a connection between aberrant DMN development and mental disorders in adulthood has been proposed (Broyd et al., 2009, Daniels et al., 2011, Northoff et al., 2011). The specific mechanisms that may underlie such a connection remain unclear, however.

Biochemically, the glutamate transmitter system is of increasing interest from a clinical perspective as glutamatergic dysfunctions appear to play a role in conditions associated with ELS, including MDD and anxiety disorders (Alcaro et al., 2010, Riaza Bermudo-Soriano et al., 2012). Studies in non-human animals have reinforced this link and have extended it to show that ELS can be a causative factor in glutamate-linked dysfunction (Ali et al., 2011, Bredy et al., 2004, Neto et al., 2011, Ryan et al., 2009, Zuena et al., 2008). More specifically, studies in humans and animals have highlighted the importance of glutamatergic alterations within the medial prefrontal cortex (mPFC) in processes underlying psychological dysfunction (amongst other regions). This holds true in the case of ELS, where preliminary studies in animals have shown an effect on the glutamatergic system within the MPFC (Ali et al., 2011, Judo et al., 2010, Llorente et al., 2012). In addition, glutamate within the mPFC - a core component of the DMN (Daniels et al., 2011) - has been linked to the intrinsic activity 
in humans (Duncan et al., 2011). The combined evidence thus provides a prima facie case for a possible link between ELS, glutamate, and the DMN, although this remains to be investigated.

The first aim of the study was thus to link ELS, as measured using the Childhood Trauma Questionnaire (CTQ - see Supplementary material E.1 for questionnaire) (Bernstein et al., 1994), and resting-state activity and biochemistry using a combination of functional MRI and magnetic resonance spectroscopy (MRS). Resting-state measures in fMRI were entropy (Bruce et al., 2009, Richman and Moorman, 2000) and the amplitude of low frequency fluctuations (Zou et al., 2008). Entropy is of particular interest as the complexity of brain activity, which entropy provides a measure of, has been shown to alter during development and has bcen linked to effective brain function (Garrett et al., 2012, Misić et al., 2010). Based on the prior work described, it was hypothesised that CTQ scores would be related to resting-state measures and correlate negatively with $\mathrm{mPFC}$ glutamate.

As ELS-related dysfunctions, such as mood and anxiety disorders, produce alterations in the brain response to aversive stimuli in humans (Etkin and Wager, 2007, Liberzon et al., 2007, Shin et al., 2004, van Tol et al., 2012), the second aim of the study was to investigate whether the suggested ELS effects on resting-state properties had functional significance for stimulus-related neural responses to aversion anticipation. Brain responses to the aversion task within the aversion network were hypothesised to correlate with CTQ scores in regions involved in aversion, such as the anterior cingulate cortex (ACC) and anterior insula (Hayes and Northoff, 2011, Ploghaus et al., 1999), with concomitant relationships with the biochemical and resting-state measures.

\subsection{Methods}

\subsubsection{Participants}

Twenty-seven healthy participants were scanned using fMRI and MRS at two different scan centres (resting-state fMRI - Montreal Neurological Institute, McGill University; aversion fMRI and MRS - Unité de neuroimagerie fonctionelle, Université de Montréal). Aversion fMRI and MRS scans were carried out on the same day. Siemens 3 Tesla Trio scanners were 


\begin{tabular}{ccc} 
& All participants $(\mathrm{n}=25)$ & MRS-rest subgroup $(\mathrm{n}=12)$ \\
\hline Age & $22 \pm 3.93$ & $23 \pm 3.5$ \\
Sex & $9 \mathrm{~F}$ & $6 \mathrm{~F}$ \\
Years in education & $13 \pm 5.69$ & $14 \pm 3.93$ \\
IQ & $113 \pm 8.42$ & $112 \pm 9.33$ \\
CTQ & $36.88 \pm 5.36$ & $37 \pm 5.08$ \\
ASI & $14.28 \pm 6.19$ & $13.57 \pm 6.57$
\end{tabular}

Table 10.1: Subject demographics and test scores (mean \pm S.D.).

used at both locations. Subjects were screened for current or past psychiatric or neurological disorders and recreational drug use (see Table 10.1 for demographic and psychological scale details.) Data from a number of participants were excluded due to incorrectly completed questionnaires, excessive head-motion in either the aversion or rest fMRI sessions, drowsiness during rest (Duncan and Northoff, 2012), or unusable MRS data. This strict quality control left a final group of twelve participants upon whom the complete analysis was performed ( 6 female, mean age $=23 \pm 3.5$ years). Results from individual components of the analysis using the maximum number of participants for each are also reported as supporting information to the twelve participant main analysis (subject numbers for each supporting analysis are given in parenthesis where relevant). All participants gave their written informed consent and were compensated financially. Approval for the study was obtained from the ethics committees at McGill University and the Université de Montréal.

\subsubsection{Psychological scales}

Participants completed the CTQ and the Anxiety Sensitivity Index (ASI) (Reiss et al., 1986). The CTQ has been shown to be stable and reliable, both in general and in the specific population studied (Bernstein et al., 1994, Paivio and Cramer, 2004). Given the relatively small sample size, total CTQ scores were used throughout. Participants also completed the Marlowe-Crowne Social Desirability Scale (SocDes), estimating the degree to which questionnaire responses were influenced by a propensity to adhere to social norms (Fraboni and Cooper, 1989). The ASI and SocDes were used as covariates in the analysis to control for confounding effects of anxiety sensitivity and questionnaire answer accuracy. 


\subsubsection{MRS}

Single voxel edited ${ }^{1} \mathrm{H}$ MR spectra were acquired using the MEGA-PRESS method (Marjanska et al., 2007, Mescher et al., 1998). Voxels of interest (VOI) were located in the mPFC $\left(48 \times 21 \times 21 \mathrm{~mm}^{3}\right)$ and the left insula $\left(23 \times 48 \times 27 \mathrm{~mm}^{3}\right)$ (see Figure 2.1 for locations). Difference spectra were analysed using LCModel 6.2-1A (Provencher, 1993, 2001) using the basis set which included an experimentally measured metabolite-nulled macromolecular spectrum from the occipital cortex (average from 10 subjects) and the experimentally measured spectra from $100 \mathrm{mM}$ phantoms of N-acetylaspartate (NAA), creatine, GABA, Glu, and Gln with $\mathrm{pH}$ adjusted to 7.2 and at $37^{\circ} \mathrm{C}$. Only results with the Cramer-Rao lower bounds (CRLB) below 20\% were included in the analysis. Concentrations with CRLB > $20 \%$ were classified as not detected. The $\mathrm{mPFC}$, centred on the perigenual anterior cingulate cortex, was the target region for the study and the left insula was used as a regional specificity control. Correlations between Glx/NAA and Glu/NAA and the variables of interest were calculated.

\subsection{4 fMRI parameters and pre-processing}

Resting-state functional EPI scans were acquired using body coil transmit and a 32-channel headcoil. Forty-seven slices aligned at -30 o from the AC-PC plane and covering the whole brain were acquired per volume $\left(\mathrm{FoV}=205 \times 205 \mathrm{~mm}^{2}\right.$; spatial resolution $=3.2 \times 3.2 \times 3.2$ $\mathrm{mm}^{3} ; \mathrm{T}_{\mathrm{E}}=25 \mathrm{~ms} ; \mathrm{T}_{\mathrm{R}}=2270 \mathrm{~ms}$; flip angle $=90^{\circ}$ ). The first five volumes were discarded. A high-resolution anatomical image was also acquired (MPRAGE; spatial resolution $=1 \times 1$ $x 1 \mathrm{~mm}^{3}$ ). Aversion task EPI scans were carried out using the same acquisition parameters as the resting-state other than the inclusion of a gap between each volume acquisition of $400 \mathrm{~ms}$. This gap was included to allow the collection of shock response EMG data without the presence of confounding scanner electromagnetic noise (Piché et al., 2010). The EMG data was not included in the present analysis.

The processing of fMRI data was carried out with the FSL suite of tools (Smith et al., 2004). In preprocessing steps common to both the rest and aversion tasks, functional images were aligned to the middle volume, slice time corrected, and high-pass filtered (100 s). Functional and anatomical images were aligned with the FSL MNI standard space 
template using non-linear transformations.

\subsubsection{Resting-state fMRI}

The resting-state session consisted of two eyes-open and two eyes-closed periods $(2 \times 120 \mathrm{~s}$ each). The order of the periods was counterbalanced across participants and was indicated with short tones. Participants were monitored using a simple camera setup to ensure that they followed the task and to monitor levels of drowsiness.

Following preprocessing, each participant's resting-state data was submitted to an independent component analysis from which noise components (head motion, blood and breathing related signals) were identified by two independent investigators (Kelly et al., 2010) and regressed out of the unsmoothed data, along with the six head-motion parameters. The primary region of interest was the MPFC MRS voxel, with the LI MRS voxel as control region. As the signal of interest for analysis was that from grey matter only, these MRS regions were individually masked to create grey matter ROIs from each. The binary grey matter masks were produced by segmenting each subject's anatomical image and thresholding the resulting grey matter maps at a probability of 0.95 . mPFC and LI grey matter ROIs were converted into functional space using the previously calculated non-linear transformations. The timecourse from each voxel within the ROIs was extracted from the unsmoothed, pre-processed data and split into $\mathrm{EO}$ and $\mathrm{EC}$ periods.

Measures of resting state activity were entropy and fALFF. Entropy for each voxel within the ROIs was calculated using the SampEn toolbox for MATLAB (http://www physionet . org/physiotools/sampen/matlab/) (Richman and Moorman, 2000) and then averaged across the whole ROI. The SampEn method gives a non-linear characterisation of the degree of organisation within a timeseries, in this case the BOLD signal (Hauge et al., 2011, Aboy et al., 2007). This is done by calculating the log likelihood that a series of points of a length $m$ (at a particular matching threshold, $r$ ) will also be the same at a length of $m+1$, as calculated for all sets of points in the timecourse. An increase in entropy is generally (though not always) associated with an increase in complexity (Costa et al., 2005). Timecourses were normalised to have a mean of 0 and variance of 1 prior to entropy calculation. A template length of $\mathrm{m}=2$ and a matching threshold of $20 \%$ of the standard deviation of the original timecourse $(r=0.2)$ was used. fALFF is a measure of the intensity of low-frequency 

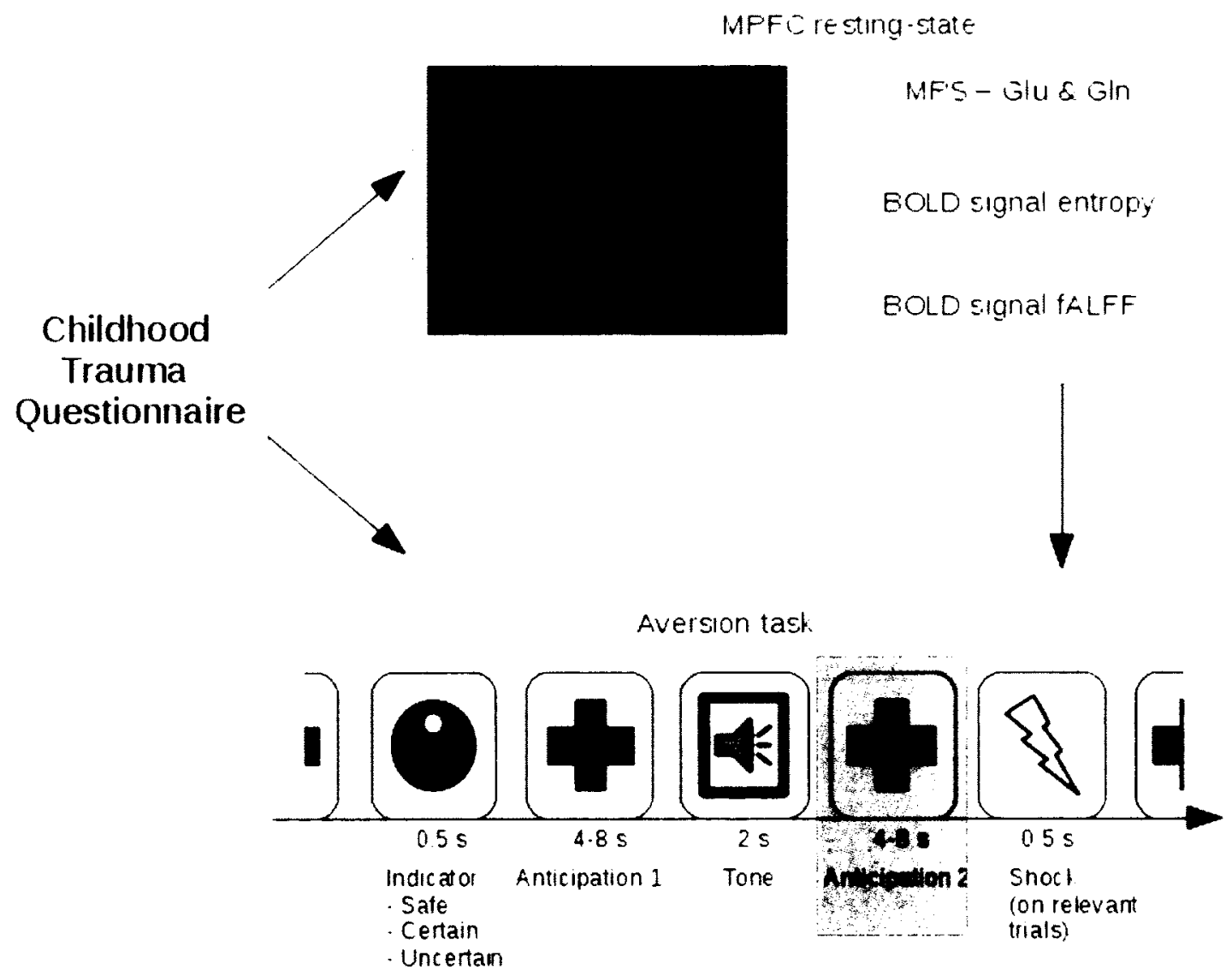

Figure 10.1: Overview of study design. The analysed period from the aversion task is highlighted in green - conditions were collapsed across tones to give simple [anticipation of certain aversion : safe] ([ACA Safe]) and [anticipation of uncertain aversion safe] ([ALA Safe]) contrasts. Arrows represent correlation analyses. 
fluctuations within a region and was calculated as described previously (Zou et al., 2008). Briefly, following preprocessing, the power of each frequency between 0.027 and $0.073 \mathrm{~Hz}$ was calculated. The sum of these square root of these powers was then divided by the sum of the square root of powers across all frequencies $(0-0.22 \mathrm{~Hz})$ to give the fALFF. Results were standardised by conversion to Z-scores.

\subsubsection{Aversion task}

Participants underwent an aversion anticipation task in which electric shocks were applied to the left ankle. Parameters for trans-cutaneous electrical stimulation were similar to those used by Ladouceur et al. (2012) and Mailhot et al. (2012). Stimulation (10 x $1 \mathrm{~ms}$ rectangular pulses; rate $=333 \mathrm{~Hz}$, train duration $=30 \mathrm{~ms} ;<25 \mathrm{~mA}$ ) was administered to the skin over the retromalleolar path of the sural nerve at the ankle via custom-made surface electrodes $(1 \mathrm{~cm} 2$; spaced $2 \mathrm{~cm}$ apart) using an isolated DS7A constant current stimulator (Digitimer, Welwyn Garden City, Hertfordshire, UK) and triggered by a Grass S88 train generator (Grass Medical Instruments, Quincy, MA, USA). Stimulation was aimed to be above the subject's pain threshold, and was determined by the staircase method (Willer, 1977), beginning with an intensity of $1 \mathrm{~mA}$ followed by increments of $1 \mathrm{~mA}$ and reaching a suprathreshold level between $15-25 \mathrm{~mA}$ (adjusted for individual reports of experienced pain). The subjective experience of pain ratings $(0-100$, where 0 is not painful and 100 is the worst imaginable pain) averaged $50.65 \pm 13.26$ across all subjects, which is consistent with mild to moderate levels of experienced pain (Mailhot et al., 2012). Real-time visual inspection of electrodermal activity was also used as further validation that the stimulation was unpleasant. The intensity of the stimulation was determined on an individual basis prior to scanning. This testing stage was carried out in a mock scanner to familiarise participants to the scanner environment.

The event-design task (Figure 10.1) focused on the anticipation of aversion and was designed to test additional hypotheses regarding the effect of context on the aversive response. At the start of each trial subjects were informed $(0.5 \mathrm{~s})$ as to whether they would receive a shock with $100 \%$ certainty (certain aversion), with $33 \%$ certainty (uncertain aversion), or whether they would receive no shock (safe). This indicator was followed by a first anticipation period (4-8 s) and then a tone presentation period (2 s). Prior to scanning, two tones were either conditioned to an aversive stimulus (i.e., white noise startle), or were 
presented in the absence of the startling noise (i.e., neutral). Following the tone was a second anticipation period (4-8 s), and finally a shock delivery in the relevant trials. The order of trials was pseudo-randomised and inter-trial intervals (1-3 s) were interspersed pseudorandomly amongst trials. Each condition was presented 25 times over four runs, giving a total scan time of approximately 50 minutes. A simplified analysis approach was used in the current study; specifically, tone types were collapsed to give only certain shock, uncertain shock, and safe conditions. Only the anticipation period following the tone presentation was analysed (Figure 10.1) as it has previously been shown that late anticipation in MRI is more sensitive to individual differences in anxiety (Carlson et al., 2011).

Following preprocessing, aversion functional data were smoothed ( $5 \mathrm{~mm}$ FWHM kernel) and then analysed using a standard GLM approach, as implemented in FEAT (Smith et al., 2004). All events were convolved with a canonical double-gamma HRF. Included in the model as nuisance variables were the six head-motion parameters and the mean signal from the CSF and white matter to minimise the effect of head movement and physiological noise. As the target was aversive responses, the contrasts of interest were the anticipation of certain aversion (ACA) versus the anticipation of no shock (Safe), [ACA v Safe], and the anticipation of uncertain aversion (AUA) versus the anticipation of no shock, [AUA $v$ Safe].

\subsubsection{Combination of measures}

Relationships between the basic measures (psychological scores, biochemistry, entropy, and fALFF values) were tested using correlation or partial correlation analyses. To determine the relationship between brain responses to the anticipation of aversion and each of the psychological scores, the entropy and fALFF values, and mPFC Glu/NAA, whole-brain regression analyses were carried out at the group level in FEAT. Significance was set at $p$ $<0.05$, FDR corrected (uncorrected threshold $p=0.009$ ). Whole-brain analyses were also corrected for multiple comparisons (cluster FWE, $\mathrm{Z}>2.3$ ). CTQ regressions included ASI scores as a confounding variable. 


\subsection{Results}

\subsubsection{CTQ \& biochemistry}

CTQ scores were positively correlated with ASI scores in the whole group $(n=25)$, controlling for subject age and SocDes scores, although this was a trend in the main $\mathrm{n}=$ 12 subgroup (Table 1a). As hypothesised, CTQ scores correlated negatively with mPFC Glu/NAA concentrations in the subgroup $(n=12)$, although not with Glx/NAA levels in the core group (Figure 10.2a; Table 10.2b). In contrast, CTQ scores were not correlated with biochemical measures in the LIns control region $(n=12)$. These results were confirmed in a supplementary analysis using all subjects with usable MRS and CTQ results $(\mathrm{n}=20)$ : CTQ scores were negatively correlated with mPFC Glu/NAA $(\mathrm{R}=-0.57, p=0.0088)$ but not correlated with $\mathrm{mPFC}$ Glx/NAA $(\mathrm{R}=-0.14, p=0.57)$, whilst in the LIns, no relationship between CTQ scores and LIns Glu/NAA $(\mathrm{R}=-0.78, p=0.75)$ or Glx/NAA ( R $=0.05, p=0.84)$ was seen.

As anxiety disorders have been linked to changes in NAA levels in the mPFC (Shin and Liberzon, 2010) and MRS results were quantified relative to this metabolite (see Methods), NAA levels in the $\mathrm{mPFC}$ were also correlated with CTQ scores. No relationship was seen $(\mathrm{n}=20, \mathrm{R}=0.11, p=0.66)$.

\subsubsection{CTQ \& resting-state FMRI}

Resting state entropy within the mPFC ROI during the eyes-closed condition was found to correlate positively with CTQ scores $(\mathrm{n}=12$; Figure $10.2 \mathrm{~b}$ ). During the EO condition, no correlation was observed. No correlation was found between the CTQ scores and entropy in the LIns during either EO or eyes-closed (Table 10.2d). No correlation was found between CTQ scores and mPFC fALFF in either the EO nor eyes-closed condition. Similarly, no correlation was seen between CTQ scores and LIns FALFF during either the EO or eyesclosed conditions. Given the correlations observed, mPFC eyes-closed entropy was also correlated with mPFC Glx/NAA and Glu/NAA, showing a negative correlation with the latter (Table 10.2d). In a maximal group of 15 participants, positive correlation between CTQ scores and mPFC eyes-closed entropy was also seen $(\mathrm{R}=0.72, p=0.0039)$, confirming 
Correlation coefficient (95\% C.I.)

\begin{tabular}{|c|c|c|}
\hline \multicolumn{3}{|l|}{ (A) [inc. age - SocDes] } \\
\hline CTQ vs ASI $(n=25)$ & \multicolumn{2}{|c|}{$0.70, p<0.001$} \\
\hline CTQ vs ASI $(n=12)$ & \multicolumn{2}{|c|}{$0.53, p=0.072$} \\
\hline (B) $n=12$ & mPFC & Left insula \\
\hline CTQ vs Glx/NAA & $-0.23(-0.74-0.33), p=0.35$ & $0.17(-0.45-0.67), p=0.67$ \\
\hline CTQ vs Glu/NAA & $-0.64(-0.91--0.23), p=0.009$ & $0.25(-0.35-0.71), p=0.40$ \\
\hline (C) $n=12$ & $\mathrm{mPFC}$ & Left insula \\
\hline CTQ vs Ent EC & $0.80(0.43-0.94), p=0.002$ & $0.25(-0.35-0.71), p=0.40$ \\
\hline CTQ vs Ent EO & $0.23(-0.33-0.68), p=0.41$ & $0.31(-0.54-0.65), p=0.33$ \\
\hline CTQ vs $\mathrm{A} A L F F$ EC & $0.35(-0.22-0.74), p=0.22$ & $0.34(-0.23-0.74), p=0.23$ \\
\hline CTQ vs fALFF EO & $0.40(-0.17-0.77), p=0.16$ & $0.40(-0.16-0.77), p=0.15$ \\
\hline Glx/NAA vs Ent EC & $-0.49(-0.83-0.11), p=0.10$ & $-0.09(-0.64-0.50), p=0.76$ \\
\hline Glu/NAA vs Ent EC & $-0.79(-0.94--0.39), p=0.002$ & $-0.17(-0.67-0.44), p=0.60$ \\
\hline
\end{tabular}

Table 10.2: Summary of correlation analyses between questionnaire scores and resting-state measures from the mPFC and left insula for eyes-open and eyes-closed rest. Significant correlations are shown in bold $\left(p_{\mathrm{FDR}}<0.05\right)$. CTQ $=$ childhood trauma questionnaire; SocDes $=$ social desirability scale; $\mathrm{ASI}=$ anxiety sensitivity index; $\mathrm{mPFC}=$ medial prefrontal cortex; Glu/NAA = glutamate $/ \mathrm{NAA}$; Glx $/$ NAA $=$ glutamate + glutamine $/$ NAA $;$ Ent $=$ sample entropy; fALFF = fractional amplitude of low frequency fluctuations.

the subgroup result.

\subsubsection{Aversion response \& CTQ, biochemistry and resting-state fMRI}

The aversion task produced responses consistent with prevous studies (Hayes and Northoff, 2011). ACA induced strong responses in, amongst other regions, the sensorimotor cortex (SMC) and bilateral insula (Figure 10.3a, Table 10.3a). AUA induced similar responses, although of a lesser extent (Supplementary figure E.2a \& table E.1). 


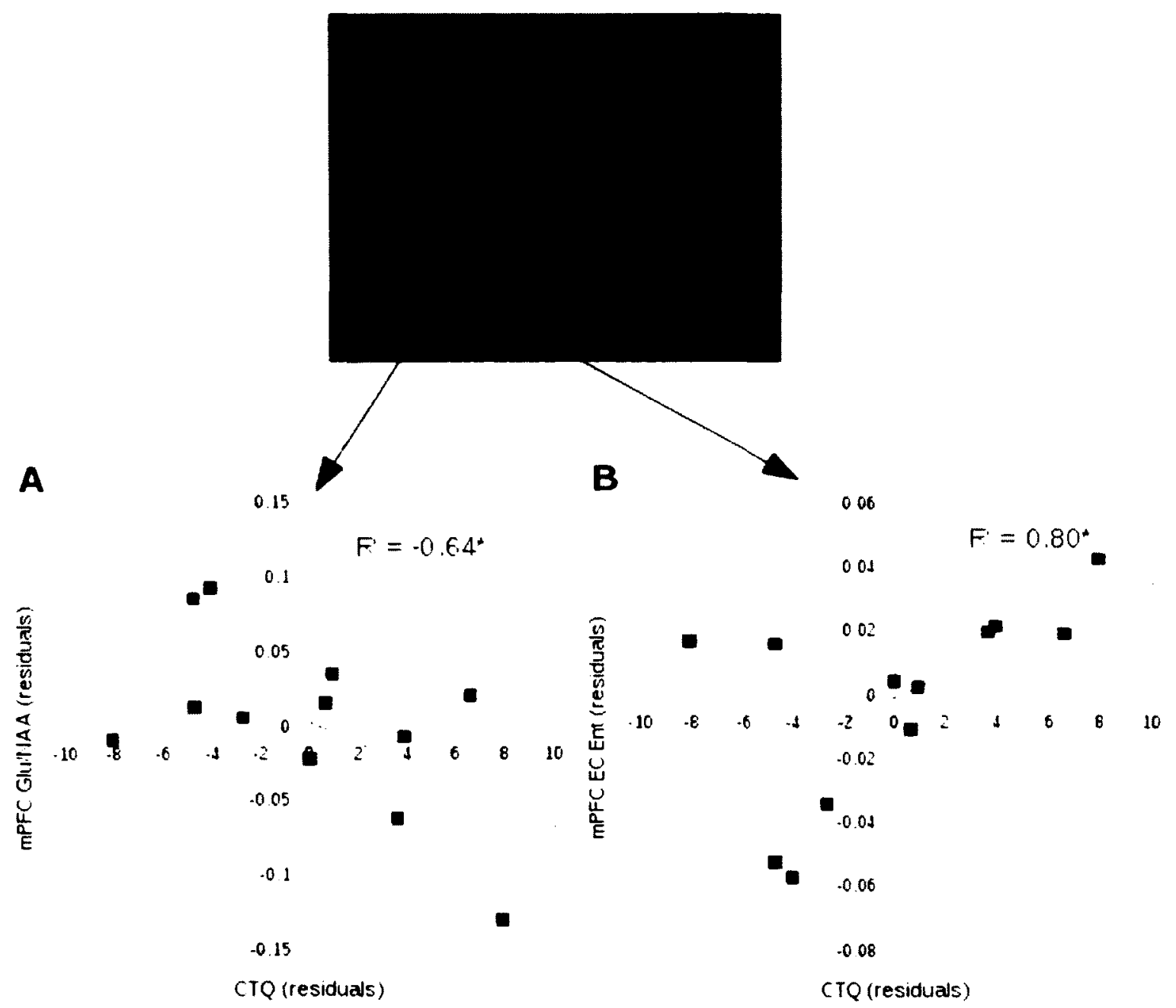

Figure 10.2: (A) Correlation between CTQ scores and mPFC Glu concentrations, and (B) between CTQ scores and mPFC entropy during eyes-closed condition, both controlling for subject age. Greater reported ELS correlates with lower Glu and higher entropy in the mPFC. * indicates $p_{\mathrm{FDR}} \cdot 0.05$. 
During $\mathrm{ACA}$, the response to the anticipation of aversion was found to correlate negatively with CTQ scores in multiple areas (controlling for ASI), primarily the PCC, SMC and left insula (Figure 10.3b, Table 10.4). The BOLD response to AUA was also found to correlate negatively with CTQ scores in the PCC and precuneus only (Supplementary figure E.2b \& table E.2).

Glu/NAA in the mPFC was found to correlate positively with the response to ACA in the SMC and left insula at a more lenient threshold of $p=0.005$, uncorrected, as opposed to the threshold of $p=0.05$, FWE corrected, used in other comparisons (Figure 10.3c, Table 10.5). This can be seen as a consequence of the higher degree of error inherent within the MRS measurements. No significant correlations with mPFC Glx/NAA, LIns Glx/NAA, and LIns Glu/NAA were seen. During AUA, no correlation was found with any of the biochemical measures at either threshold.

Entropy in the $\mathrm{mPFC}$ during EC was found to correlate negatively with ACA responses in the insula and SMC (Figure 10.3d, Table 10.6). No relationship with mPFC entropy in the eyes-open condition was found. Similarly, no relationship was found with mPFC fALFF during either the eyes-open or eyes-closed condition. The response to AUA was found to correlate negatively with $\mathrm{mPFC}$ eyes-closed entropy in the SMC and precuneus (Supplementary figure E.2d \& table E.3). No relationship was found between AUA and mPFC eyes-open entropy, mPFC eyes-open or eyes-closed fALFF.

Results from each of the regressions with ACA activity were combined to visually identify regions in which all overlap, highlighting the motor cortex and left insula (Figure 10.3e). To further typify the CTQ effect on aversion responses in these overlapping regions, signal changes for the individual conditions (Safe, AUA, and ACA) were extracted from the motor cortex overlap. These were then correlated with CTQ scores, showing that the negative correlation with $[\mathrm{ACA}>\mathrm{Safe}]$ activity is driven by a reduced ACA response, rather than increased Safe signal changes (Figure 10.3f).

\subsection{Discussion}

Resting-state fMRI and MRS data were combined with an MRI aversion task and the CTQ to investigate the relationship between ELS and brain function in healthy adults. 

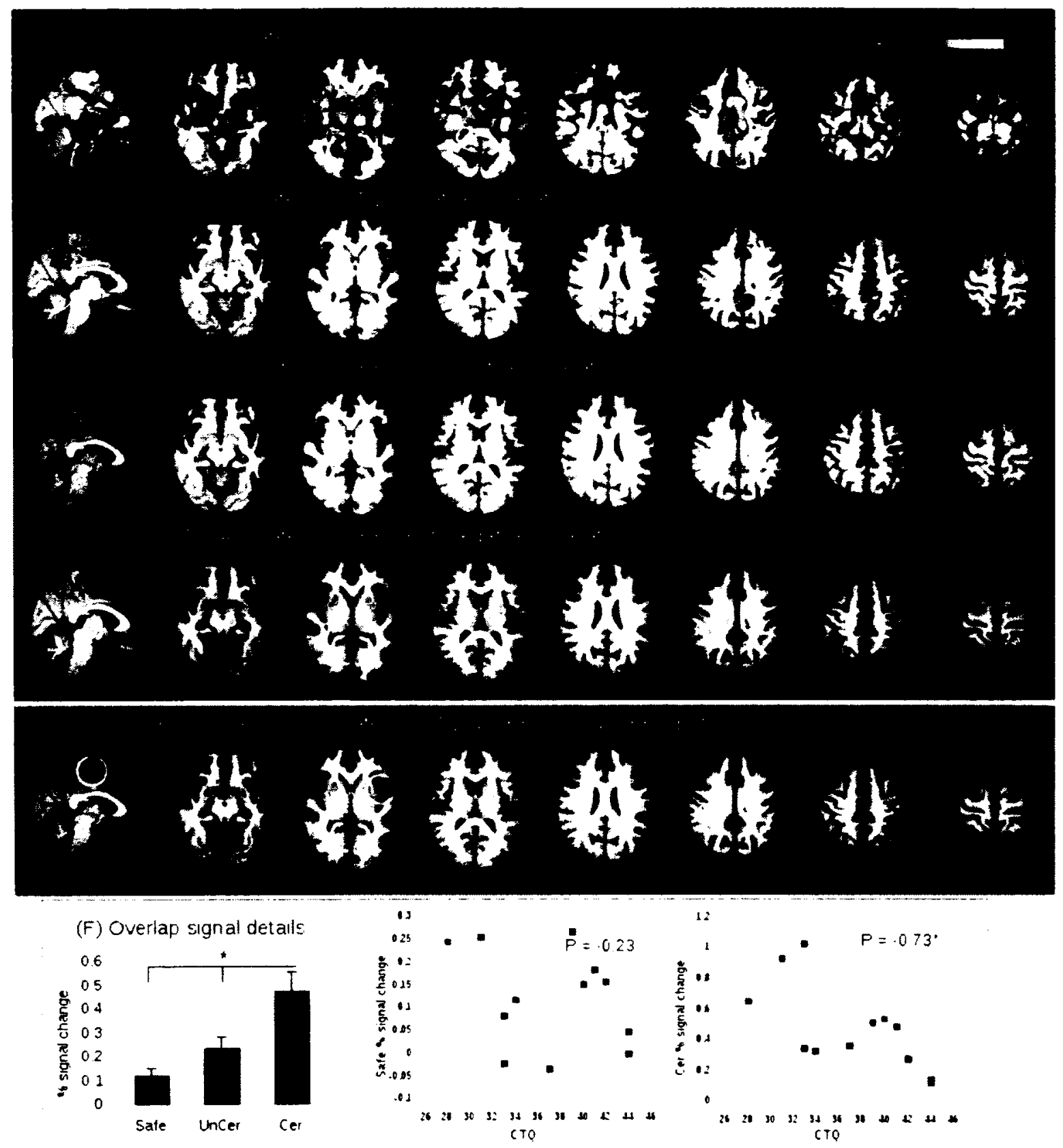

Figure 10.3: Brain maps showing (A) the basic contrast of the anticipation of certain aversion : safe [ACA Safe]; (B) the negative correlation between [ACA Safe] contrast values and CTQ scores (controlling for ASI): (C) the positive correlation between [ACA - Safe] contrast values and mPFC Glu; (D) the negative correlation between [ACA Safe] contrast values and $m P F C$ entropy: and $(E)$ the overlap, in green, between each of these maps. Details of Safe and Certain percent signal changes from the overlap are given, showing that CTQ scores are correlated with a decrease in Certain sigual change (F). Image result threshold is $p<0.05$, FWE corrected $(\mathrm{n}=12)$, other than $[\mathrm{ACA}$. Safe] vs mPFC Glu/NAA where it is $p<0.005$, uncorrected. Results are shown superimposed on the study mean anatomical image. ${ }^{*}$ indicates $p_{\mathrm{FDR}} \cdot 0.05$. Sagittal $\mathrm{x} \quad$ 4. CTQ $=$ childhood trauma questionnaire; mPFC - medial prefrontal cortex: Glu/NAA - glutamate/NAA; Ent $=$ sample entropy. 


\begin{tabular}{|c|c|c|c|c|}
\hline Region & & $\mathrm{x}, \mathrm{y}, \mathrm{z}$ & voxels & Z-max \\
\hline \multirow[t]{2}{*}{ post-insula/HG } & $\mathbf{R}$ & $\begin{array}{lll}38 & -20 & 12\end{array}$ & 22676 & 6.36 \\
\hline & $\mathrm{L}$ & $-34-2214$ & & \\
\hline \multirow[t]{2}{*}{ ant-insula/operculum } & $\mathbf{R}$ & 3848 & & \\
\hline & $\mathrm{L}$ & -3606 & & \\
\hline \multirow[t]{2}{*}{$\mathrm{POC}$} & $\mathrm{L}$ & $-48-4022$ & & \\
\hline & $\mathrm{R}$ & $\begin{array}{lll}42 & -28 & 18\end{array}$ & & \\
\hline cerebellum & $L / R$ & $-6-46-22$ & & \\
\hline \multirow[t]{2}{*}{ thalamus } & $\mathrm{L}$ & $-18-226$ & & \\
\hline & $\mathrm{R}$ & $14-14-6$ & & \\
\hline mid-insula & $\mathrm{L}$ & $-38-8-8$ & & \\
\hline post-SMG & $\mathrm{R}$ & $58-30 \quad 30$ & & \\
\hline \multirow[t]{2}{*}{ vs } & $\mathrm{L}$ & -1200 & & \\
\hline & $\mathrm{R}$ & $144-2$ & & \\
\hline \multirow[t]{2}{*}{ amygdala } & $\mathbf{R}$ & $242-16$ & & \\
\hline & $L$ & $-18-4-16$ & & \\
\hline \multirow[t]{2}{*}{$\mathrm{SMC}$} & $\mathrm{R}$ & $12-4072$ & 11916 & 6.31 \\
\hline & L. & $-16-3256$ & & \\
\hline \multirow[t]{2}{*}{$\mathrm{MCC} / \mathrm{ACC}$} & $\mathrm{L}$ & -12636 & & \\
\hline & $\mathrm{R}$ & 6436 & & \\
\hline precuneus & $\mathrm{L} / \mathrm{R}$ & $-10-4858$ & & \\
\hline \multirow[t]{2}{*}{$\mathrm{PCC}$} & $L / R$ & $0-2046$ & & \\
\hline & & $-10-2038$ & & \\
\hline $\mathrm{MCC} / \mathrm{SMA}$ & $L / R$ & $-2-442$ & & \\
\hline $\mathrm{ACC}$ & $L / R$ & 42218 & & \\
\hline paracingulate gyrus & $\mathrm{L} / \mathrm{R}$ & 61452 & & \\
\hline cerebellum & & $-20-44-52$ & 257 & 5.09 \\
\hline brain-stem & $\mathrm{L} / \mathrm{R}$ & $-2-36-40$ & 199 & 4.71 \\
\hline \multirow[t]{2}{*}{$1^{\circ} \mathrm{MC}$} & L & $-40-1048$ & 195 & 4.22 \\
\hline & $L / R$ & $-38-2038$ & & \\
\hline \multirow[t]{2}{*}{$2^{\circ}$ visual } & $\mathrm{L}$ & $22-602$ & 137 & 4.34 \\
\hline & $\mathrm{L} / \mathrm{R}$ & $\begin{array}{llll}22 & -70 & 10\end{array}$ & & \\
\hline
\end{tabular}

Table 10.3: Results for contrast [ACA > Safe]. Coordinates are in MNI space. Significance threshold is $p<0.05$, FWE corrected $(\mathrm{Z}>2.3)$. SMC = sensory-motor cortex; $\mathrm{SMA}=$ supplementary motor area; $\mathrm{HG}=$ Herschl's gyrus; $1^{\circ} \mathrm{MC}=$ primary motor cortex; $2^{\circ}$ visual $=$ secondary visual cotex; $\mathrm{ACC}=$ anterior cingulate cortex $; \mathrm{POC}=$ parietal operculum cortex $\mathrm{VS}=$ ventral striatum $; \mathrm{MCC}=$ midcingulate cortex; $\mathrm{ACC}=$ anterior cingulate cortex; $\mathrm{PCC}=$ posterior cingulate cortex 


\begin{tabular}{ccccc} 
Region & & $\mathrm{x}, \mathrm{y}, \mathrm{z}$ & voxels & Z-max \\
\hline S.MC/PCC/precuneus/SMA & $\mathrm{L} / \mathrm{R}$ & $-10-2476$ & 3533 & 3.5 \\
post-SMG & $\mathrm{R}$ & $56-4444$ & 860 & 3.27 \\
dmPFC/ACC & $\mathrm{R}$ & 105228 & 526 & 3.3 \\
operculum/mid-insula/post-insula & $\mathrm{L}$ & $-46-2418$ & 1365 & 3.35
\end{tabular}

Table 10.4: Cluster locations for [ACA vs Safe] vs CTQ regression. Note that the thalamus cluster is mostly in the right thalamus. Coordinates are in MNI space. Threshold is $p<0.05$, FWE corrected $(\mathrm{Z}>2.3)$. SMC = sensory-motor cortex; PCC $=$ posterior cingulate cortex; SMA = supplementary motor area; Post-SMG = posterior supramarginal gyrus; $\mathrm{dmPFC}=$ dorsomedial prefrontal cortex; ACC = anterior cingulate cortex.

\begin{tabular}{ccccc} 
Region & & $\mathrm{x}, \mathrm{y}, \mathrm{z}$ & voxels & Z-max \\
\hline SMC & L/R & $4-1650$ & 133 & 3.4 \\
post-SMG & $\mathrm{L}$ & $-64-5036$ & 99 & 3.3 \\
insula/operculum & $\mathrm{L}$ & -4428 & 67 & 3.36
\end{tabular}

Table 10.5: Cluster locations for [ACA vs Safe] vs mPFC Glu/NAA regression. Coordinates are in MNI space. Threshold is $p<0.005$, uncorrected $(\mathrm{k}>50)$. SMC $=$ sensory-motor cortex; Post-SMG = posterior supramarginal gyrus.

\begin{tabular}{ccccc} 
Region & & $\mathrm{x}, \mathrm{y}, \mathrm{z}$ & voxels & $\mathrm{Z}$-max \\
\hline SMC/SMA & $\mathrm{L} / \mathrm{R}$ & $-2-1852$ & 933 & 4.18 \\
operculum/mid-insula & $\mathrm{L}$ & $-568-4$ & 627 & 3.73 \\
1o MC & $\mathrm{L}$ & $-38-1852$ & 611 & 3.44
\end{tabular}

Table 10.6: Cluster locations for [ACA vs Safe] vs mPFC Ent regression. Coordinates are in MNI space. Threshold is $p<0.05$, FWE corrected $(\mathrm{Z}>2.3)$. $\mathrm{SMC}=$ sensory-motor cortex; SMA $=$ supplementary motor area; $1^{\circ} \mathrm{MC}=$ primary motor cortex. 
CTQ scores were found to correlate with the resting-state level of Glu/NAA in the mPFC, as well as with the degree of entropy of the resting-state BOLD signal in the same region. CTQ scores were further found to correlate with the brain responses to the anticipation of aversion in multiple regions, including the ACC, S.MC and insula. This relationship between CTQ and aversion responses was shown to overlap with correlations between aversion responses and $\mathrm{mPFC}$ Glu levels and entropy in the SMC and insula. Taken together, these results show that ELS may have a lasting impact on resting-state activity in the MPFC via glutamatergic changes and that this alteration of resting-state properties correlates with the neural response to aversive stimuli.

ELS was found to correlate with $\mathrm{MPFC}$ resting-state MRS values, with a greater degree of ELS (as measured by the CTQ) linked with lower levels of Glu/NAA. No relationship between $\mathrm{CTQ}$ scores and Glu/NAA in the insula control region was seen, implying regional specificity for the mPFC. Numerous studies in non-human animals have demonstrated an effect of ELS on glutamatergic function in the mPFC (Ali et al., 2011, Llorente et al., 2012, Matrisciano et al., 2011)(along with other regions (Neto et al., 2011, Ryan et al., 2009, Waes et al., 2009)). More specifically, MRS glutamatergic measures in the mPFC (as measured by MRS) have been shown in rats to be reduced by ELS (Llorente et al., 2012), along with reduced mPFC firing rates (Ali et al., 2011), and altered NMDA receptor expression coupled with NMDA-linked impaired synaptic potentiation (Ali et al., 2011, Judo et al., 2010, Matrisciano et al., 2011). The observed changes due to ELS are also in line with a general link between impaired glutamatergic function in the $\mathrm{MPFC}$ and animal models of acute and long-term stress and anxiety (Gutièrrez-Mecinas et al., 2011, Harvey and Shahid, 2012, Knox et al., 2010). No previous studies have linked ELS and glutamatergic function in humans; however, many studies have investigated glutamate in the context of mood and anxiety disorders, consistently finding alterations across the brain and a specific reduction in glutamate levels in the mPFC (Alcaro et al., 2010, Hettema et al., 2011, Riaza Bermudo-Soriano et al., 2012).

Alterations in intrinsic activity, particularly in the DMN, have been linked to mood and anxiety disorders that are also associated with ELS (Broyd et al., 2009, Northoff et al., 2011, Sylvester et al., 2012). The current findings advance this by demonstrating that ELS has a direct effect on resting-state activity properties in the $\mathrm{MPFC}$, a key region within the DMN (Daniels et al., 2011). More specifically, greater ELS was shown to increase the degree of entropy in mPFC activity during eyes-closed rest. This connection was specific to the 


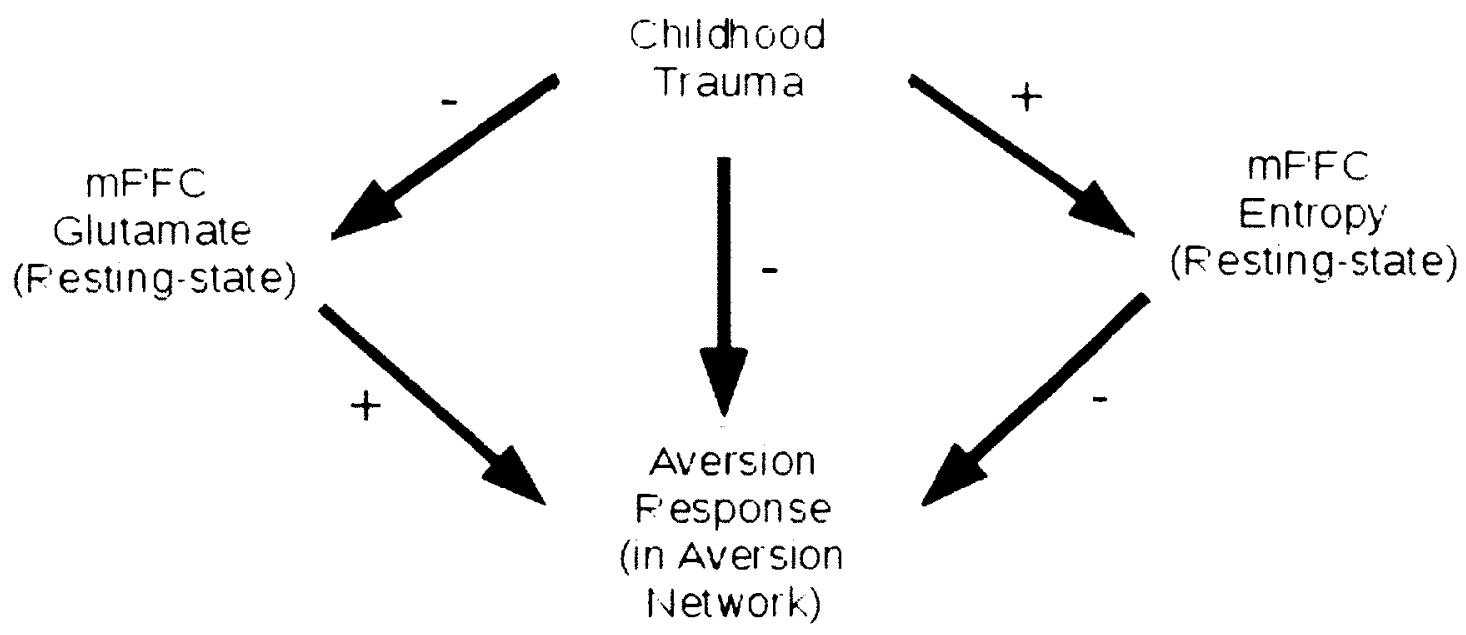

Fignere 10.4: Overview of study results showing relationship between CTQ scores. restingstate measures, and aversion task responses. Green arrows denote a positive relationship and red negative. mPFC medial prefrontal cortex.

InPFC and to entropy, as compared to the insula and fALFF. Sample ent ropy represents the degree of organisation within the mPFC activity over time; the link between entropy and glutamate seen here thus suggests that this transmitter plays a role in structuring mPFC activity and that this relationship can be affected by ELS. These findings on glutamate, ELS. and entropy complement and extend previous studies that have shown an effect of ELS on EEG frequency bands that in turn predict signal entropy. (Bruce et al., 2009. McFarlane et al., 2005), as well as prior results demonstrating an effect of life-stress on mPFC restingstate activity (Molina et al., 2010. Shin and Handwerger, 2009). In addition, they highlight that the dynamic properties of intrinsic activity that develop over childhood are likely to be important for healthy adult brain function (McIntosh et al., 2008. Uhlhaas and Singer, 2011).

Brain responses to the anticipation of aversion were shown to be negatively correlated with CTQ scores in both ACA and ACA, with a more extensive relationship during ACA (all controlling for ASI). This means that with increasing CTQ scores there is a decrease in the difference in activation between the aversive (ACA or ACA) and safe conditions. Focusing on ACA, ELS was found to correlate with activity within the arersion network (Hayes 
and Northoff, 2011), and in particular in the SMC and left insula. This correlation with CTQ-scores was mirrored by correlations with mPFC Glu/NAA and eyes-closed entropy (Figure 4), demonstrating that ELS effects on resting-state properties have concurrent effects on aversion-related function. Although the majority of previous work has focused on abnormally increased regional activations in ELS-related mood and anxiety disorders, often highlighting the role of the amygdala and hippocampus (Dannlowski et al., 2012a, Hart and Rubia, 2012), a recent meta-analysis of PTSD imaging studies found decreased activation in many of the regions highlighted in the negative correlation between ACA and CTQ scores (Etkin and Wager, 2007). Similarly, prior work has shown that, in line with the current results, higher anxiety levels correlate with reduced activity changes in response to aversion in the regions identified (Drevets et al., 1995, Simpson et al., 2001, Zhao et al., 2007). In addition, it has been demonstrated that glutamatergic measures in the mPFC correlates positively with BOLD responses in task-positive regions (Duncan et al., 2011), fitting with the relationship seen between Glu/NAA and aversion response. Taken in conjunction with the changes in intrinsic activity described above, the current results would suggest that ELS leads to lowered aversion-induced activity changes in multiple brain regions, and that this may be linked to glutamate-related intrinsic activity levels.

Interestingly, the amygdala, which has been closely linked to the effects of ELS (Dannlowski et al., 2012b,a), ELS-related disorders such as MDD (Siegle et al., 2007, Victor et al., 2010) and PTSD (Shin et al., 2005, Francati et al., 2007), and to aversive processing itself (Hayes and Northoff, 2011, Shin and Liberzon, 2010), does not feature in the current results when correlating aversive responses with CTQ scores (nor with mPFC entropy or glutamate); the amygdala is shown to be active during the anticipation of aversion, however, as would be expected (see Table 10.3). More specifically, ELS has been linked with increased amygdala responsiveness (Dannlowski et al., 2012a), and so a positive correlation between CTQ scores and aversion-related activity may have been expected. The lack of a correlation between aversive responses and CTQ scores seen here may be a result of the whole-brain approach adopted, contrasting with the region of interest analysis employed in the majority of studies linking ELS and amygdala changes in humans (for example, Dannlowski et al., 2012b,a)(see also Hart and Rubia, 2012, for a critical review of amygdala imaging studies in child abuse). The different statistical limitations of these two approaches may thus mean that an effect of ELS on aversive responses in the amygdala may not be apparent in the current work, but would be were the former analysis approach to be adopted. This presents an interesting 
avenue for future research, in particular where an analysis of the interaction between the mPFC and amygdala in the context of ELS may be carried out (Burghy et al., 2012). Also of note is the fact that when compared, no sex differences are seen in the aversive brain responses (data not shown). This contrasts with previous work suggesting that the effect of ELS on the brain differs between males and females (Salzberg et al., 2007, Weinstock, 2007, Duchesne et al., 2009, Burghy et al., 2012). In the current work, the small sample size for each sex (six each of male and female) may be limiting the ability to detect differences between them and so a larger sample is required to establish what, if any, gender effects there are in this context. Of final note is the consideration as to whether the ELS-related changes in Glu/Cr levels (plus related entropy and aversion responses) suggested by the correlation with CTQ scores are pathological or whether they reflect a form of resilience (Cisler et al., 2012). In the first case, the negative correlation between CTQ scores and $\mathrm{Glu} / \mathrm{Cr}$ concentrations would represent a deleterous reduction in transmitter as a result of ELS, with this deficit then translating into disrupted neural and psychological performance, albeit at a subclinical level. Some support for this interpretation comes from the positive correlation between CTQ and ASI scores (see Table 10.2). With the latter interpretation, in contrast, the fact that the participants are all healthy can be taken as support for the lower transmitter levels with higher CTQ scores reflecting a potential protective aspect of reduced mPFC Glu/Cr. Should the same levels of ELS have occured in people with a higher preexisting Glu/Cr concentration, they may, according to this interpretation, have proceeded to develop clinical symptoms. Some form of longitudinal study is, however, required to differentiate entirely between these two possibilities.

A number of limitations of the current study must be noted. Firstly, the final number of subjects with the full set of usable $\mathrm{MRI}$ and MRS data was not large. Although consistency of these results with those of the larger subgroups (see Supplementary material) and the generally good levels of significance obtained point to their robustness, replication with a larger sample is required. Secondly, one may question the reliability of CTQ self-report; however, the evidence from prior research into the validity of abuse reports would suggest that they are reliable (Chu et al., 1999). In a similar vein, participants were selected to have had no psychiatric problems in the past and to be healthy at the time of the study, so one may question the validity of concluding brain changes from CTQ scores in a non-clinical population. Prior studies have, however, observed physiological changes correlating with CTQ scores in healthy subjects (Carpenter et al., 2011, McFarlane et al., 2005). That this 
is the case could be seen as representing a strength of the current study, as the observation of an effect in a non-clinical population demonstrates the sensitivity of the brain to ELS and the potential continuum-like nature of ELS effects. Finally, subjects were recruited from the local student population and so, given the general class and educational structure of such a group, may not be representative of the population as a whole. Extending the study into different social contexts and specific patient populations is thus a desirable next step.

In conclusion, using a unique multimodal imaging dataset, it was shown that ELS impacts upon $\mathrm{mPFC}$ resting-state dynamics and biochemistry, and that these changes are related to altered responses to the anticipation of aversion. These results provide a link between previous animal studies and clinical observations and give insight into the mechanisms by which early-life experiences may impact upon brain function in adulthood.

\subsection{Acknowledgements}

Many thanks to A. Perna and K. Dedovic for their help with subject recruitment, Edward J. Auerbach, Ph.D. (Centre for Magnetic Resonance Research, University of Minnesota) for implementing MEGA-PRESS sequence on Siemens, the staff at the UNF and MNI for their skillful assistance, and Romain Valabregue, Ph.D. (Centre de NeuroImagerie de Recherche, Paris, France) for developing processing tools. The work was supported by grants to G.N. from the Canadian Institutes of Health Research and the Michael Smith Foundation. 


\section{Chapter 11}

\section{Discussion}

In the preceding pages we have tried to approach a number of questions relating to the linkage between the structural, functional and process-level domains in order to begin to piece together the biochemical underpinnings of the inter-related networks identified in each. A range of imaging techniques were employed to this end, including functional magnetic resonance imaging (fMRI), magnetic resonance spectroscopy (MRS), diffusion tensor imaging (DTI), positron emission tomography (PET), and electroencephalography (EEG).

It was shown firstly that medial prefrontal cortex (mPFC) glutamate levels correlate with structural and functional connectivity to multiple cortical and subcortical regions during both rest and task. It was further shown that glutamate in this region is correlated with the signal dynamics of the ongoing activity and that this relationship changes with the transition from eyes-closed to eyes-open rest. Secondly, it was shown that cortical GABA $A$ receptor distribution follows similar topologies to structural and functional connectivity, fitting the description of "small-worldness". It was further shown that the distribution of $\mathrm{GABA}_{\mathrm{A}}$ receptors can be decomposed into distinct networks that correspond to known functional and structural ones. Thirdly, using fMRI and DTI, it was shown that the structural network profile of the insula predicts its activity during a specific task, and that the different structural networks identified corresponded well with previously established ones. Finally, it was shown that ongoing activity dynamics in adulthood are related to early-life stress, and that the dynamical differences seen across individuals were related to mPFC glutamate levels. It was further shown that these early-life stress related changes are correlated with altered neural responses to aversive stimuli.

Out of these results, further insight into the role of entropy in brain function, as discussed in Chapters 4, 5 and 10, can be highlighted. Physiological signal complexity - as measured 
here using sample entropy - is a factor in biological processes ranging from, for example, overall bodily movement over time (Hauge et al., 2011, Pittman and McAlpine, 2003) to cardiovasular activity (Berger et al., 2012, Kim et al., 2013) and mechanoreception (Douglass et al., 1993, Pfeiffer and French, 2009), with changes in these signal structures have been linked to disease states (Berger et al., 2012, Gómez et al., 2009, Hauge et al., 2011, Leistedt et al., 2011). In the context of neural activity, multiple studies have shown that a capacity for variability is necessary for the efficient functioning of the brain (Deco et al., 2011, Mcdonnell and Ward, 2011). For example, greater variability is correlated with improved performance in a reaction time task (McIntosh et al., 2008), in visual matching (Raja Beharelle et al., 2012), or in attention tasks (Garrett et al., 2012). It has been suggested that this capacity for variability is necessary to allow shifting between the multiple brain states possible in response to ongoing changes in internal and external task demands (Misić et al., 2010). However, it seems that a "healthy" range of variability potential exists, with too much, such as in dementia (Abásolo et al., 2006), being detrimental as much as too little, such as in epilepsy or schizophrenia (Rosso, 2007, Winterer et al., 2006). Thus, with too little complexity in the brain's intrinsic activity, brain states may be pathologically constrained to remain within a narrow and non-flexible set; with too much, a potential for inefficient switching outwith the relevant task demands may occur, again leading to pathological functioning. The current work adds to these ideas in two main ways. Firstly, the link shown between mPFC glutamate and entropy (a measure of complexity) provides a first link in humans between ongoing activity structure and the brain's biochemistry. This helps underscore that what is being measured is not extraneous noise but is instead linked to neural function, as well as providing clues as to the processes underlying brain variability (Deco and Jirsa, 2012). Secondly, the link between entropy levels and ELS, as measured using the CTQ, adds weight to the idea that there is a "healthy" range of variability as the assumedly pathological experiences of early life trauma are correlated with a change in complexity. We would thus expect to see those with higher levels of ELS that result in clinical symptoms to be located further out on this continuum.

In addition, some interesting areas of convergence can be seen amongst the work reported. Firstly, the importance of network interactions was highlighted across a number of experiments. In Chapter 2 it was shown that functional and structural connectivity between the cortex and subcortical regions can be identified in humans in vivo, reflecting closely existing knowledge of such cortical-subcortical networks obtained through non-human an- 
imal studies. Additionally, task-relevant network interactions were described in Chapters 3 and 10 in the context of empathy and aversion, respectively. At a structural level, it was shown in Chapter 9 that connectivity to distributed networks of regions influences activity within the insula during tasks. Network interactions were also potentially identified at the receptor level in Chapter 8, where correlated changes in $\mathrm{GABA}_{\mathrm{A}}$ binding potential across subjects were shown to give rise to dissociable networks that closely reflect known functional and structural ones and share similar formal properties to them. These multiple layers of evidence for a network-based organisation in the brain fits with an view of it that can be described as "associationist" (Catani et al., 2012), where dissociable large scale networks are devoted to particular functions but overall brain function derives from a dynamic interaction between these networks. Working along these lines, the cortical and subcortical networks identified at a functional, structural and biochemical level (see Chapter 2) would thus represent parts of individual large scale networks, whilst the different structural networks that predict insula functional responses in each of intero- and exteroception (see Chapter 9) would represent the dynamic switching in network associations depending on task demands.

Secondly, the role of glutamate in $\mathrm{mPFC}$ intrinsic activity was consistently revealed over two separate analyses. In Chapter 4 it was shown that increasing $\mathrm{Glx} / \mathrm{Cr}$ concentrations in this region were correlated with an increase in fixation period activity (taken as an operationalised rest period), as measured in a general linear model. This was hypothesised to be due to a reduction in variance across the period leading to an improved model fit. In Chapter 5 it was then shown that increasing Glu/NAA concentrations in the same region were correlated with a reduction in resting-state entropy. Increasing glutamate concentrations in the mPFC were thus independently linked to more ordered ongoing activity in both studies. A related point of convergence across studies was the consistent finding that increasing glutamate concentrations in the $\mathrm{mPFC}$ were correlated with increased taskinduced BOLD responses in separate task-relevant regions. In Chapter 3 it was shown that increasing $\mathrm{Glx} / \mathrm{Cr}$ concentrations in the $\mathrm{MPFC}$ were correlated with more activity in the caudal anterior cingulate (cACC) in response to an empathy task (a known empathyrelated region (Fan et al., 2011)). In a separate experiment, it was shown in Chapter 10 that increased Glu/NAA concentrations in the mPFC were correlated with more activity in multiple aversion-related regions (Hayes and Northoff, 2011) in response to the anticipation of an aversive stimulus. 
A third area of convergence is inter-related findings regarding connections between the insula and $\mathrm{CACC}$. In Chapter 3 , a correlation between left insula responses and $\mathrm{Glx} / \mathrm{Cr}$ concentrations in the $\mathrm{CACC}$ was shown during an empathy task. At the same time, the impact of structural connectivity between these two regions on activity in the insula in response to interoception was demonstrated in Chapter 9. As interoception and empathy as neural processes have both been closely linked to the insula (Chang et al., 2012, Gu et al., 2012, Oosterwijk et al., 2012, Wiebking et al., 2011), this structural and biochemical overlap between the two tasks is suggestive. Additionally, in Chapter 8 it was shown that the cACC and insula are both members of the same specific $\mathrm{GABA}_{\mathrm{A}}$ receptor network, adding another potential layer of convergence amongst findings in these regions. These two regions have been suggested to for what has been labelled the "salience network" (Menon and Uddin, 2010, Seeley et al., 2007). This network is proposed to act in identifying which stimuli out of the multiple co-occuring internal and external inputs that the brain receives are the most relevant to the maintainance of homeostasis. On this account, the salience network is involved in driving the dynamic switches between externally-aligned, action networks and more internally-aligned interospective networks as appropriate, based on current inputs. The current works presents the first information as to the biochemistry underlying such a network by demonstrating both the involvement of glutamate in communication between the regions and by identifying a distinct $\mathrm{GABA}_{\mathrm{A}}$ receptor network containing them. In the latter case it is more likely that it is convergent modulation of receptor density based on coactivations that is being observed, rather than GABAergic signalling between the two regions.

Finally, the context sensitivity of ongoing activity was shown across different experiments. The transition from eyes-closed to eyes-open rest was shown to alter ongoing activity properties across the brain in Chapter 5, and in the mPFC specifically in Chapters 2, 5, and 3. In Chapter 2 it was shown that glutamate and structural correlations between the mPFC and a set of subcortical regions were altered by this change in context, with correlations that were seen in the eyes-closed condition no longer holding in the eyes-open, and vice versa. Similarly, in Chapter 5 it was shown that linear and non-linear dynamic properties of ongoing activity change between eyes-open and eyes-closed rest and that this is linked to altered coupling with glutamate concentrations in the mPFC, whilst in Chapter 3 it was shown that task context altered the relationship between ongoing activity in the mPFC and activity changes in other regions, with these relationships altering between the viewing 
and appraisal of emotional pictures. Taking a wider contextual perspective, it was shown in Chapter 10 that ongoing activity properties in the mPFC are correlated with life experiences, with greater reported childhood trauma being correlated with greater entropy in the $\mathrm{mPFC}$ at rest, with a concurrent reduction in Glu/NAA concentrations in the same region. These early-life stress related changes in ongoing activity were further correlated with reported anxiety and activity changes in response to an aversion task.

These interactions between context and ongoing activity at short and long timescales are suggestive of a fundamental role for the kind of ongoing networks studied here. A number of suggestions as to what this role may be have been put forward, all of which have a fairly consistant kernel Carhart-Harris and Friston (2010), Hesselmann et al. (2010), Northoff et al. (2010b), Raichle (2009), Sadaghiani et al. (2010). Each of us receives stimulus from the outside world that is objectively the same. The light waves reflected from an object have identical physical properties regardless of whose retina they hit, for instance. Yet our experience of these stimuli can be radically different. The same item can be experienced as neutral by one person whilst instilling joy in another. Some process must therefore act to add subjective colour and meaning to these objective stimuli - they must be contextualised according to some individual "habit" or "custom" (Hume, 1748). The sensitivity of ongoing activity to being shaped by life experiences that is described here, such that individual neural responses to an objective stimuli can be shown to be correlated with such variance, may thus mean that the activity can be described as one substrate for such habits. Support for such a hypothesis comes from research showing that structural and functional networks develop in complex and process-relevant manners from childhood to adulthood (Cohen Kadosh, 2011, Dennis et al., 2012, Lebel et al., 2008, McIntosh et al., 2008, Uddin et al., 2010, 2011) and that the course that this development takes is related to subjective experience in adulthood (Cohen Kadosh, 2011, Dannlowski et al., 2012b,a). In addition, one's cultural context has been shown to affect neural and perceptual responses to objective stimuli (Athanasopoulos et al., 2010, Kitayama and Park, 2010, Winawer et al., 2007). However, in addition to such developmental evidence, the strength of such a view of intrinsic structural and functional properties shaping subjective experience is that it is not bound to timeframes that are, in the context of the objective world we are responding to, so short. The biochemical and structural underpinnings of the properties in question have been shaped over massively longer timescales by evolution, meaning that appropriate habits need not arise within the limits of a single human's experience, but can instead be shaped and passed 
down over eons, representing a tempting repository for those a posteriori associations that complex life requires a priori in order to surivive in the world from birth.

Further potential evidence for such a role for ongoing activity comes from the links to psychiatric conditions that can be drawn and which are described in each chapter. For example, Chapter 2 draws links between the patterns of cortical-subcortical connectivity described and changes seen in schizophrenia and depression, whilst the structural connectivity networks described in Chapter 9 are put into the context of structural changes seen in depression. If ongoing activity networks provide a background upon which incoming stimuli are given their content or colour, a change in these networks would in turn produce a change in the perceived properties of stimuli. In the case of depression, for example, a stimulus that was previously held to be rewarding could shift with changes in the background reference to being neutral or even aversive Northoff et al. (2011). Similarly, in a condition such as schizophrenia, stimuli that should be innoculous could become alien and threatening should the ongoing activity patterns with which they interact change Northoff and Qin (2011).

With this hypothesised link with psychiatric disorders, an interesting future direction for the work presented here would be to apply the same approaches to patient groups. In a related manner, the glutamatergic relationships described and the posited functionallyrelevant $\mathrm{GABA}_{\mathrm{A}}$ receptor networks could be tested through glutamate and $\mathrm{GABA}$ challenge studies in order to show more concrete links (rather than the purely correlational links shown here). For example, the use of a glutamatergic antagonist such as ketamine would allow the role of glutamate in cortical-subcortical network communication, as discussed in Chapter 2, to be tested, or for the same transmitter's role in ongoing activity dynamics (as discussed in Chapters 4 and 5) to be clarified. Similarly, the use of GABAergic substances, such as valproic acid, over suitable timescales would allow the testing of the proposed functional nature of $\mathrm{GABA}_{\mathrm{A}}$ receptor networks described in Chapter 8. A pharmacological challenge approach would also allow closer conclusions to be drawn between the findings reported here and known biochemical deficits in conditions such as depression. Finally, as described in many of the chapters, translational work in non-human animals would allow potential causative descriptions of the phenomena reported here to be made and would allow a bridging of the gap between indirect imaging techniques such as BOLD fMRI and direct measures of neural firing. 
To conclude, the work presented here provides initial biochemical links between structural and functional networks in the brain. The evidence presented extends the network conception of the brain to a biochemical level for the first time in humans, providing a base for future studies involving GABA and glutamate (and the interplay between these). In addition, the biochemical underpinnings of ongoing activity were described and were shown, in conjunction with structural properties, to be relevant to individual responses to different tasks. This linkage between biochemistry, intrinsic activity and task-related activity adds to prior work investigating the role of intrinsic activity in psychological function and provides the first evidence that glutamate, for one, is a transmitter involved in rest-stimulus interactions. Finally, the work provides some insights into the organising principals of the brain in action and gives promising clues for the investigation of a number of psychiatric disorders, particularly depression and schizophrenia. 


\section{References}

Sargo Aalto, Jouni Ihalainen, Jussi Hirvonen, Jaana Kajander, Harry Scheinin, Heikki Tanila, Kjell Nå gren, Harry Vilkman, Lars L Gustafsson, Erkka Syvälahti, and Jarmo Hietala. Cortical glutamate-dopamine interaction and ketamine-induced psychotic symptoms in man. Psychopharmacology, 182(3):375-83, November 2005. ISSN 0033-3158. doi: 10.1007/s00213-005-0092-6. URL http://www.ncbi.nlm.nih.gov/pubmed/16001106.

D. Abásolo, R. Hornero, P. Espino, D. Alvarez, and J. Poza. Entropy analysis of the EEG background activity in Alzheimer's disease patients. Physiological Measurement, 27(3):241-53, March 2006. ISSN 0967-3334. doi: 10.1088/0967-3334/27/3/003. URL http://www.ncbi.nlm.nih.gov/pubmed/16462011http://iopscience.iop.org/ $0967-3334 / 27 / 3 / 003$.

M. Aboy, D. Cuesta-Frau, D. Austin, and P. Mico-Tormos. Characterization of sample entropy in the context of biomedical signal analysis. In Conf Proc IEEE Eng Med Biol Soc, pages 5942-5945. IEEE, 2007. URL http://ieeexplore. ieee.org/xpls/abs_all. jsp?arnumber $=4353701$.

Sophie Achard and Ed Bullmore. Efficiency and cost of economical brain functional networks. PLoS Computational Biology, 3(2):e17, February 2007. ISSN 15537358. doi: 10.1371/journal.pcbi.0030017. URL http://www.pubmedcentral.nih.gov/ articlerender.fcgi?artid=1794324\&tool=pmcentrez\&rendertype=abstract.

Sophie Achard, Raymond Salvador, Brandon Whitcher, John Suckling, and Ed Bullmore. A resilient, low-frequency, small-world human brain functional network with highly connected association cortical hubs. The Journal of Neuroscience, 26(1):6372, January 2006. ISSN 1529-2401. doi: 10.1523/JNEUROSCI.3874-05.2006. URL http://www.ncbi.nlm.nih.gov/pubmed/16399673. 
Antonio Alcaro, Jaak Panksepp, Jan Witczak, Dave J Hayes, and Georg Northoff. Is subcortical-cortical midline activity in depression mediated by glutamate and GABA? A cross-species translational approach. Neuroscience and Biobehavioral Reviews, 34(4): 592-605, March 2010. ISSN 1873-7528. doi: 10.1016/j.neubiorev.2009.11.023. URL http://www.ncbi.nlm.nih.gov/pubmed/19958790.

Aaron F Alexander-Bloch, Nitin Gogtay, David Meunier, Rasmus Birn, Liv Clasen, Francois Lalonde, Rhoshel Lenroot, Jay Giedd, and Edward T Bullmore. Disrupted modularity and local connectivity of brain functional networks in childhood-onset schizophrenia. Frontiers in Systems Neuroscience, 4:147, January 2010. ISSN 1662-5137. doi: 10.3389/fnsys.2010.00147. URL http://www.pubmedcentral.nih.gov/articlerender. fcgi?artid=2965020\&tool=pmcentrez\&rendertype=abstract.

I Ali, MR Salzberg, C French, and NC Jones. Electrophysiological insights into the enduring effects of early life stress on the brain. Psychopharmacology, 214(1):155-173, 2011. URL http://www.springerlink.com/index/KM8P2G30W8765G64.pdf.

X An, R Bandler, D Ongür, and J L Price. Prefrontal cortical projections to longitudinal columns in the midbrain periaqueductal gray in macaque monkeys. The Journal of Comparative Neurology, 401(4):455-79, November 1998. ISSN 0021-9967. URL http: //www.ncbi.nlm.nih.gov/pubmed/9826273.

Amit Anand, Yu Li, Yang Wang, Jingwei Wu, Sujuan Gao, Lubna Bukhari, Vincent $\mathrm{P}$ Mathews, Andrew Kalnin, and Mark J Lowe. Activity and connectivity of brain mood regulating circuit in depression: a functional magnetic resonance study. Biological Psychiatry, 57(10):1079-88, May 2005. ISSN 0006-3223. doi: 10.1016/j.biopsych.2005.02.021. URL http://www.ncbi.nlm.nih.gov/pubmed/15866546.

IL Arancibia-Carcamo and JT Kittler. Regulation of GABA A receptor membrane trafficking and synaptic localization. Pharmacology \& Therapeutics, 123:17-31, 2009. URL http://ww. sciencedirect.com/science/article/pii/S0163725809000618.

A Arieli, A Sterkin, A Grinvald, and A Aertsen. Dynamics of ongoing activity: explanation of the large variability in evoked cortical responses. Science, 273(5283):1868-71, September 1996. ISSN 0036-8075. URL http://www.ncbi.nlm.nih.gov/pubmed/8791593. 
Adam R Aron. The neural basis of inhibition in cognitive control. The Neuroscientist, 13 (3):214-28, June 2007. ISSN 1073-8584. doi: 10.1177/1073858407299288. LRL http: //ww.ncbi.nlm.nih.gov/pubmed/17519365.

$\mathrm{J}$ Ashburner and $\mathrm{K} \mathrm{J}$ Friston. Nonlinear spatial normalization using basis functions. Human Brain Mapping, 7(4):254-66, January 1999. ISSN 1065-9471. URL http://www.ncbi. nlm.nih.gov/pubmed/10408769.

Amber Asher and Daniel J Lodge. Distinct prefrontal cortical regions negatively regulate evoked activity in nucleus accumbens subregions. The International Journal of Neuropsychopharmacology, pages 1-8, October 2011. ISSN 1469-5111. doi: 10.1017/ S146114571100143X. URL http://www.ncbi.nlm.nih.gov/pubmed/22008178.

Sina Aslan, Hao Huang, Jinsoo Uh, Virendra Mishra, Guanghua Xiao, Matthias J P van Osch, and Hanzhang Lu. White matter cerebral blood flow is inversely correlated with structural and functional connectivity in the human brain. NeuroImage, 56(3):1145-53, June 2011. ISSN 1095-9572. doi: 10.1016/j.neuroimage. 2011.02.082. URL http://ww. pubmedcentral.nih.gov/articlerender.fcgi?artid= 3085605\&tool=pmcentrez\&rendertype=abstract .

Panos Athanasopoulos, Benjamin Dering, Alison Wiggett, Jan-Rouke Kuipers, and Guillaume Thierry. Perceptual shift in bilingualism: brain potentials reveal plasticity in preattentive colour perception. Cognition, 116(3):437-43, September 2010. ISSN 1873-7838. doi: 10.1016/j.cognition.2010.05.016. URL http://www.ncbi.nlm.nih.gov/pubmed/ 20566193.

D.L. Bailey, D.W. Townsend, P.E. Valk, and M.N. Maisey, editors. Positron Emission Tomography: Basic Sciences. Springer, Secaucus, NJ, 1st edition, 2003.

Albert-László Barabási. Scale-free networks: a decade and beyond. Science, 325(5939): 412-3, July 2009. ISSN 1095-9203. doi: 10.1126/science.1173299. URL http://www . ncbi.nlm.nih.gov/pubmed/19628854.

Robert J Barry, Adam R Clarke, Stuart J Johnstone, Christopher a Magee, and Jacqueline a Rushby. EEG differences between eyes-closed and eyes-open resting conditions. Clinical 
Neurophysiology, 118(12):2765-73, December 2007. ISSN 1388-2457. doi: 10.1016/j. clinph.2007.07.028. LRL http://www.ncbi.nlm.nih.gov/pubmed/17911042.

Robert J Barry, Adam R Clarke, and Stuart J Johnstone. Caffeine and opening the eyes have additive effects on resting arousal measures. Clinical Neurophysiology, 122(10): 2010-2015, April 2011. ISSN 1872-8952. doi: 10.1016/j.clinph.2011.02.036. URL http: //www.ncbi.nlm.nih.gov/pubmed/21489866.

Paolo Bartolomeo. The quest for the 'critical lesion site' in cognitive deficits: problems and perspectives. Cortex, 47(8):1010-2, September 2011. ISSN 1973-8102. doi: 10.1016/j. cortex.2010.11.007. URL http://www.ncbi.nlm.nih.gov/pubmed/21185556.

A.T. Beck, R.A. Steer, R. Ball, and W. Ranieri. Comparison of Beck Depression Inventories -IA and -II in psychiatric outpatients. Journal of Personality Assessment, 67(3):588-97, 1996.

Christian F Beckmann, Marilena DeLuca, Joseph $\mathrm{T}$ Devlin, and Stephen M Smith. Investigations into resting-state connectivity using independent component analysis. Philosophical Transactions of the Royal Society of London. Series B, Biological Sciences, 360(1457):1001-13, May 2005. ISSN 0962-8436. doi: 10.1098/rstb. 2005.1634. URL http://www.pubmedcentral.nih.gov/articlerender.fcgi?artid= 1854918\&tool=pmcentrez\&rendertype=abstract.

Matthias Beckmann, Heidi Johansen-Berg, and Matthew F S Rushworth. Connectivitybased parcellation of human cingulate cortex and its relation to functional specialization. The Journal of Neuroscience, 29(4):1175-90, January 2009. ISSN 15292401. doi: 10.1523/JNEUROSCI.3328-08.2009. URL http://www.ncbi.nlm.nih.gov/ pubmed/19176826.

MD De Bellis and SR Hooper. Neuropsychological findings in childhood neglect and their relationships to pediatric PTSD. Journal of the International Neuropsychological Society, 15(6):868-78, 2009. URL http://journals.cambridge.org/production/action/ cjoGetFull text?fulltextid $=6097104$.

MD De Bellis, MS Keshavan, H Shifflett, S Iyengar, SR Beers, J Hall, and G Moritz. Brain structures in pediatric maltreatment-related posttraumatic stress disorder: a so- 
ciodemographically matched study. Biological Psychiatry, 52(11):1066-78, 2002. URL http://www.sciencedirect.com/science/article/pii/s0006322302014592.

Sandy Berger, Anna Kliem, Vikram Yeragani, and Karl-Jürgen Bär. Cardio-respiratory coupling in untreated patients with major depression. Journal of affective disorders, 139 (2):166-71, July 2012. ISSN 1573-2517. doi: 10.1016/j.jad.2012.01.035. URL http: //www.ncbi.nlm.nih.gov/pubmed/22386048.

DP Bernstein, L Fink, L Handelsman, J Foote, M Lovejoy, K Wenzel, E Sapareto, and J Ruggiero. Initial reliability and validity of a new retrospective measure of child abuse and neglect. The American Journal of Psychiatry, 151(8):1132-6, 1994. URL http: //psycnet.apa.org/psycinfo/1995-00092-001.

Anders Björklund and Stephen B Dunnett. Dopamine neuron systems in the brain: an update. Trends in Neurosciences, 30(5):194-202, May 2007. ISSN 0166-2236. doi: 10. 1016/j.tins.2007.03.006. URL http://www.ncbi.nlm.nih.gov/pubmed/17408759.

Robyn L Bluhm, Peter C Williamson, Elizabeth a Osuch, Paul a Frewen, Todd K Stevens, Kristine Boksman, Richard W J Neufeld, Jean Théberge, and Ruth a Lanius. Alterations in default network connectivity in posttraumatic stress disorder related to earlylife trauma. Journal of Psychiatry \& Neuroscience, 34(3):187-94, May 2009. ISSN 1488-2434. URL http://www.pubmedcentral.nih.gov/articlerender.fcgi?artid= $2674971 \&$ tool=pmcentrez\&rendertype=abstract.

M Boly, E Balteau, C Schnakers, C Degueldre, G Moonen, A Luxen, C Phillips, P Peigneux, $\mathrm{P}$ Maquet, and S Laureys. Baseline brain activity fluctuations predict somatosensory perception in humans. Proceedings of the National Academy of Sciences of the United States of America, 104(29):12187-92, July 2007. ISSN 0027-8424. doi: 10.1073/pnas. 0611404104. URL http://ww.pubmedcentral nih.gov/articlerender.fcgi?artid= 1924544\&tool=pmcentrez\&rendertype=abstract.

Melanie Boly, Christophe Phillips, Evelyne Balteau, Caroline Schnakers, Christian Degueldre, Gustave Moonen, Andre Luxen, Philippe Peigneux, Marie-Elisabeth Faymonville, Pierre Maquet, and Steven Laureys. Consciousness and cerebral baseline activity fluctuations. Human Brain Mapping, 29(7):868-74, July 2008. ISSN 1097-0193. doi: 
10.1002/hbm.20602. URL http://ww.ncbi.nlm.nih.gov/pubmed/18465800.

Emre Bora, Alex Fornito, Christos Pantelis, and Murat Yücel. Gray matter abnormalities in Major Depressive Disorder: A meta-analysis of voxel based morphometry studies. Journal of Affective Disorders, April 2011. ISSN 1573-2517. doi: 10.1016/j.jad.2011.03.049. URL http://ww. ncbi.nlm.nih.gov/pubmed/21511342.

Ahmet Bozkurt, Karl Zilles, Axel Schleicher, Lars Kamper, Ernesto Sanz Arigita, Harry B M Uylings, and Rolf Kötter. Distributions of transmitter receptors in the macaque cingulate cortex. NeuroImage, 25(1):219-29, March 2005. ISSN 1053-8119. doi: 10.1016/ j.neuroimage.2004.10.040. URL http://www.ncbi.nlm.nih.gov/pubmed/15734357.

TW Bredy, TY Zhang, RJ Grant, J Diorio, and MJ Meany. Peripubertal environmental enrichment reverses the effects of maternal care on hippocampal development and glutamate receptor subunit expression. European Journal of Neuroscience, 20(5):135562, 2004. URL http://onlinelibrary.wiley.com/doi/10.1111/j.1460-9568.2004. $03599 . x / f u l 1$.

JD Bremner, M Vythilingam, E Vermetten, SM Southwick, T McGlashan, A Nazeer, S Khan, LV Vaccarino, R Soufer, PK Garg, CK Ng, LH Staib, JS Duncan, and DS Charney. MRI and PET study of deficits in hippocampal structure and function in women with childhood sexual abuse and posttraumatic stress disorder. American Journal of Psychiatry, 160(5):924-32, 2003. URL http://ajp.psychiatryonline.org/article. aspx?articleID $=176214$.

Steven L Bressler and Vinod Menon. Large-scale brain networks in cognition: emerging methods and principles. Trends in Cognitive Sciences, 14(6):277-90, June 2010. ISSN 1879-307X. doi: 10.1016/j.tics.2010.04.004. URL http://ww .ncbi.nlm.nih.gov/ pubmed/20493761.

Samantha J Broyd, Charmaine Demanuele, Stefan Debener, Suzannah K Helps, Christopher J James, and Edmund J S Sonuga-Barke. Default-mode brain dysfunction in mental disorders: a systematic review. Neuroscience and Biobehavioral Reviews, 33 (3):279-96, March 2009. ISSN 0149-7634. doi: 10.1016/j.neubiorev.2008.09.002. URL http://www.ncbi.nlm.nih.gov/pubmed/18824195. 
E.N. Bruce, M.C. Bruce, and S. Vennelaganti. Sample entropy tracks changes in EEG power spectrum with sleep state and aging. Journal of Clinical Neurophysiology, 26(4):257, 2009. doi: 10.1097/WNP.0b013e3181b2f1e3.Sample. URL http://www.sciencemag.org/content/306/5703/1895. shorthttp://www.ncbi. nlm.nih.gov/pmc/articles/PMC2736605/.

Randy L Buckner, Jessica R Andrews-Hanna, and Daniel L Schacter. The brain's default network: anatomy, function, and relevance to disease. Annals of the New York Academy of Sciences, 1124:1-38, March 2008. ISSN 0077-8923. doi: 10.1196/annals.1440.011. URL http://www.ncbi.nlm.nih.gov/pubmed/18400922.

Edward T Bullmore and Danielle S Bassett. Brain graphs: graphical models of the human brain connectome. Annual Review of Clinical Psychology, 7:113-40, January 2011. ISSN 1548-5951. doi: 10.1146/annurev-clinpsy-040510-143934. URL http://www.ncbi.nlm. nih.gov/pubmed/21128784.

Cory a Burghy, Diane E Stodola, Paula L Ruttle, Erin K Molloy, Jeffrey M Armstrong, Jonathan a Oler, Michelle E Fox, Andrea S Hayes, Ned H Kalin, Marilyn J Essex, Richard J Davidson, and Rasmus M Birn. Developmental pathways to amygdalaprefrontal function and internalizing symptoms in adolescence. Nature Neuroscience, November 2012. ISSN 1097-6256. doi: 10.1038/nn.3257. URL http://ww . nature.com/ doifinder/10.1038/nn. 3257 .

Niko A Busch, Julien Dubois, and Rufin VanRullen. The phase of ongoing EEG oscillations predicts visual perception. The Journal of Neuroscience, 29(24):7869-76, June 2009. ISSN 1529-2401. doi: 10.1523/JNEUROSCI.0113-09.2009. URL http://www.ncbi.nlm.nih. gov/pubmed/19535598.

Ryan K Butler, Linda Nilsson-Todd, Carine Cleren, Isabelle Léna, René Garcia, and David P Finn. Molecular and electrophysiological changes in the prefrontal cortexamygdala-dorsal periaqueductal grey pathway during persistent pain state and fearconditioned analgesia. Physiology \& Behavior, 104(5):1075-81, October 2011. ISSN 1873-507X. doi: 10.1016/j.physbeh.2011.05.028. URL http://www.ncbi.nlm.nih.gov/ pubmed/21683728. 
Richard B Buxton. Interpreting oxygenation-based neuroimaging signals: the importance and the challenge of understanding brain oxygen metabolism. Frontiers in Neuroenergetics, 2(8):1-16, January 2010. ISSN 1662-6427. doi: 10.3389/fnene. 2010.00008. URL http://ww .pubmedcentral.nih.gov/articlerender.fcgi?artid= 2899519\&tool=pmcentrez\&rendert ype=abstract.

$\mathrm{R} \mathrm{L}$ Carhart-Harris and $\mathrm{K} \mathrm{J}$ Friston. The default-mode, ego-functions and free-energy: a neurobiological account of Freudian ideas. Brain, 133(Pt 4):1265-83, April 2010. ISSN 1460-2156. doi: 10.1093/brain/awq010. URL http://ww. pubmedcentral.nih.gov/ articlerender.fcgi? artid=2850580\&tool=pmcentrez\&rendertype=abstract.

JM Carlson, T Greenberg, D Rubin, and LR Mujica-Parodi. Feeling anxious: anticipatory amygdalo-insular response predicts the feeling of anxious anticipation. Social Cognitive and Affective Neuroscience, 6:74-81, 2011. URL http://scan.oxfordjournals.org/ content $/ 6 / 1 / 74$.short.

LL Carpenter, TT Shattuck, AR Tyrka, TD Geracioti, and LH Price. Effect of childhood physical abuse on cortisol stress response. Psychopharmacology, 214(1):367-375, 2011. URL http://www . springerlink. com/index/R12626J26313H562. pdf.

Cameron S Carter and Vincent van Veen. Anterior cingulate cortex and conflict detection: an update of theory and data. Cognitive, Affective $\&$ Behavioral Neuroscience, 7(4):36779, December 2007. ISSN 1530-7026. URL http://www.ncbi.nlm.nih.gov/pubmed/ 18189010 .

Mauricio Castillo, Lester Kwock, and Suresh K. Mukherji. Clinical applications of proton MR spectroscopy. American Journal of Neuroradiology, 17:1-15, 1996. URL http: //www.ajnr.org/content/17/1/1.short.

Marco Catani, Flavio Dell Acqua, Alberto Bizzi, Stephanie Forkel, Steve Williams, Andy Simmons, Declan Murphy, and Michel Thiebaut de Schotten. Beyond cortical localization in clinico-anatomical correlation. Cortex, pages 1-26, August 2012. ISSN 00109452. doi: 10.1016/j.cortex.2012.07.001. URL http://linkinghub.elsevier.com/retrieve/pii/ S0010945212002328.

Franco Cauda, Federico DAgata, Katiuscia Sacco, Sergio Duca, Giuliano Geminiani, and 
Alessandro Vercelli. Functional connectivity of the insula in the resting brain. NeuroImage, 55(1):8-23, March 2011. ISSN 1095-9572. doi: 10.1016/j.neuroimage.2010.11.049. URL http://www.ncbi.nlm.nih.gov/pubmed/21111053.

Franco Cauda, Diana M-E. Torta, Katiuscia Sacco, Elisabetta Geda, Federico DAgata, Tommaso Costa, Sergio Duca, Giuliano Geminiani, and Martina Amanzio. Shared Core Areas between the Pain and Other Task-Related Networks. PLoS ONE, 7(8):e41929, August 2012. ISSN 1932-6203. doi: 10.1371/journal.pone.0041929. URL http://dx. plos .org/10.1371/journal. pone.0041929.

Luke J Chang, Tal Yarkoni, Mel Win Khaw, and Alan G Sanfey. Decoding the Role of the Insula in Human Cognition: Functional Parcellation and Large-Scale Reverse Inference. Cerebral Cortex, March 2012. ISSN 1460-2199. doi: 10.1093/cercor/bhs065. URL http://www.ncbi.nlm.nih.gov/pubmed/22437053.

Jean-Lon Chen, Tomas Ros, and John H. Gruzelier. Dynamic changes of ICA-derived EEG functional connectivity in the resting state. Human Brain Mapping, February 2012. ISSN 10659471. doi: 10.1002/hbm.21475. URL http://doi.wiley.com/10.1002/hbm. 21475.

Kalina Christoff. Undirected thought: neural determinants and correlates. Brain research, 1428:51-9, January 2012. ISSN 1872-6240. doi: 10.1016/j.brainres.2011.09.060. URL http://www.ncbi.nlm.nih.gov/pubmed/22071565.

JA Chu, LM Frey, BL Ganzel, and JA Matthews. Memories of childhood abuse: Dissociation, amnesia, and corroboration. American Journal of Psychiatry, 156(5):74955, 1999. URL http://ajp.psychiatryonline.org/article . aspx?Volume=156\&amp; page $=749$ \&amp ; journalID $=13$.

J M Cisler, G a James, S Tripathi, T Mletzko, C Heim, X P Hu, H S Mayberg, C B Nemeroff, and C D Kilts. Differential functional connectivity within an emotion regulation neural network among individuals resilient and susceptible to the depressogenic effects of early life stress. Psychological Medicine, pages 1-12, July 2012. ISSN 1469-8978. doi: 10.1017/S0033291712001390. URL http://ww.ncbi.nlm.nih.gov/pubmed/22781311.

Sarah M Clinton and James H Meador-Woodruff. Thalamic dysfunction in schizophrenia: neurochemical, neuropathological, and in vivo imaging abnormalities. Schizophrenia Re- 
search, 69(2-3):237-53, August 2004. ISSN 0920-9964. LRL http://www.ncbi.nlm.nih . gov/pubmed/15469196.

Lauren L. Cloutman, Richard J. Binney, Mark Drakesmith, Geoffrey J.M. Parker, and Matthew a. Lambon Ralph. The variation of function across the human insula mirrors its patterns of structural connectivity: Evidence from in vivo probabilistic tractography. NeuroImage, 59(4):3514-3521, November 2011. ISSN 10538119. doi: 10.1016/j.neuroimage.2011.11.016. URL http://linkinghub.elsevier.com/retrieve/ $\mathrm{pii} / \mathrm{S} 1053811911012973$.

Alexander L Cohen, Damien A Fair, Nico U F Dosenbach, Francis M Miezin, Donna Dierker, David C Van Essen, Bradley L Schlaggar, and Steven E Petersen. Defining functional areas in individual human brains using resting functional connectivity MRI. NeuroImage, 41(1):45-57, May 2008. ISSN 1053-8119. doi: 10.1016/j.neuroimage. 2008.01.066. URL http://ww. pubmedcentral.nih.gov/articlerender.fcgi?artid= 2705206\&tool=pmcentrez\&rendertype=abstract.

Kathrin Cohen Kadosh. What can emerging cortical face networks tell us about mature brain organisation? Developmental Cognitive Neuroscience, 1(3):246-55, July 2011. ISSN 1878-9307. doi: 10.1016/j.den.2011.02.001. URL http://ww.ncbi.nlm.nih.gov/ pubmed/22436510.

David M Cole, Stephen M Smith, and Christian F Beckmann. Advances and pitfalls in the analysis and interpretation of resting-state FMRI data. Frontiers in Systems Neuroscience, 4(April):8, January 2010. ISSN 1662-5137. doi: 10.3389/fnsys. 2010.00008. URL http://ww.pubmedcentral.nih.gov/articlerender.fcgi?artid= 28545318 tool=pmcentrez\&rendertype=abstract.

Christine E Collins, David C Airey, Nicole a Young, Duncan B Leitch, and Jon H Kaas. Neuron densities vary across and within cortical areas in primates. Proceedings of the National Academy of Sciences of the United States of America, 107(36):15927-32, September 2010. ISSN 1091-6490. doi: 10.1073/pnas.1010356107. URL http://www pubmedcentral nih. gov/articlerender fcgi?artid=2936588\&tool=pmcentrez\&rendertype=abstract.

Corrado Corti, John Henry Xuereb, Luca Crepaldi, Mauro Corsi, Francesca Michielin, and 
Francesco Ferraguti. Altered levels of glutamatergic receptors and $\mathrm{Na}-/ \mathrm{K}+$ ATPase$\alpha 1$ in the prefrontal cortex of subjects with schizophrenia. Schizophrenia Research, 128 (1-3):7-14, May 2011. ISSN 1573-2509. doi: 10.1016/j.schres.2011.01.021. URL http: //ww.ncbi.nlm.nih.gov/pubmed/21353485.

Madalena Costa, Ary Goldberger, and C.-K. Peng. Multiscale entropy analysis of biological signals. Physical Review E, 71(2):1-18, February 2005. ISSN 1539-3755. doi: 10.1103/ PhysRevE.71.021906. URL http://link.aps.org/doi/10.1103/PhysRevE.71.021906.

N. Costes, A. Dagher, K. Larcher, A.C. Evans, D.L. Collins, and A. Reilhac. Motion correction of multi-frame PET data in neuroreceptor mapping: Simulation based validation. Neuroimage, 47(4):1496-1505, 2009. URL http://www.sciencedirect.com/science/ article/pii/s1053-8119(09)00558-8.

J T Coull. Neural correlates of attention and arousal: insights from electrophysiology, functional neuroimaging and psychopharmacology. Progress in Neurobiology, 55(4):34361, July 1998. ISSN 0301-0082. URL http://www.ncbi.nlm.nih.gov/pubmed/9654384.

Márcio Ramos Coutinho and Leda Menescal-de Oliveira. Role of homocysteic acid in the guinea pig (Cavia porcellus) anterior cingulate cortex in tonic immobility and the influence of NMDA receptors on the dorsal PAG. Behavioural Brain Research, 208(1):237-42, March 2010. ISSN 1872-7549. doi: 10.1016/j.bbr.2009.11.047. URL http://ww.ncbi.nlm.nih.gov/pubmed/19963012.

R Cameron Craddock, G Andrew James, Paul E Holtzheimer, Xiaoping P Hu, and Helen S Mayberg. A whole brain ARI atlas generated via spatially constrained spectral clustering. Human Brain Mapping, 1928:1914-1928, July 2011. ISSN 1097-0193. doi: 10.1002/hbm.21333. URL http://www.ncbi.nlm.nih.gov/pubmed/21769991.

A D Craig. How do you feel? Interoception: the sense of the physiological condition of the body. Nature Reviews Neuroscience, 3(8):655-66, August 2002. ISSN 1471-003X. doi: 10.1038/nrn894. URL http://www.ncbi.nlm.nih.gov/pubmed/12154366.

A D Craig. How do you feel-now? The anterior insula and human awareness. Nature Reviews Neuroscience, 10(1):59-70, January 2009. ISSN 1471-0048. doi: 10.1038/nrn2555. URL http://www.ncbi.nlm.nih.gov/pubmed/19096369. 
Hugo D Critchley. Neural mechanisms of autonomic, affective, and cognitive integration. The Journal of Comparative Neurology, 493(1):154-66, December 2005. ISSN 0021-9967. doi: $10.1002 / c n e .20749$. URL http://www.ncbi.nlm.nih.gov/pubmed/16254997.

Hugo D Critchley, Stefan Wiens, Pia Rotshtein, Arne Ohman, and Raymond J Dolan. Neural systems supporting interoceptive awareness. Nature Neuroscience, 7(2):189-95, February 2004. ISSN 1097-6256. URL http://www.ncbi.nlm.nih.gov/pubmed/14730305.

Will $\mathrm{J}$ Cronenwett and John Csernansky. Thalamic pathology in schizophrenia. Current Topics in Behavioral Neurosciences, 4:509-28, January 2010. ISSN 1866-3370. URL http://www.ncbi.nlm.nih.gov/pubmed/21312411.

$\mathrm{J} L \mathrm{Cummings.} \mathrm{Anatomic} \mathrm{and} \mathrm{behavioral} \mathrm{aspects} \mathrm{of} \mathrm{frontal-subcortical} \mathrm{circuits.} \mathrm{Annals} \mathrm{of}$ the New York Academy of Sciences, 769:1-13, December 1995. ISSN 0077-8923. URL http://www.ncbi.nlm.nih.gov/pubmed/8595019.

J S Damoiseaux, C F Beckmann, E J Sanz Arigita, F Barkhof, Ph Scheltens, C J Stam, S M Smith, and S a R B Rombouts. Reduced resting-state brain activity in the "default network" in normal aging. Cerebral Cortex, 18(8):1856-64, August 2008. ISSN 1460-2199. doi: 10.1093/cercor/bhm207. URL http://www.ncbi.nlm.nih.gov/pubmed/18063564.

Judith K Daniels, Paul Frewen, Margaret C McKinnon, and Ruth a Lanius. Default mode alterations in posttraumatic stress disorder related to early-life trauma: a developmental perspective. Journal of Psychiatry \& Neuroscience, 36(1):56-9, January 2011. ISSN 1488-2434. doi: 10.1503/jpn.100050. URL http://www.pubmedcentral.nih.gov/ articlerender.fcgi ?artid=3004976\&tool=pmcentrez\&rendertype=abstract.

Udo Dannlowski, Harald Kugel, Franziska Huber, Anja Stuhrmann, Ronny Redlich, Dominik Grotegerd, Katharina Dohm, Christina Sehlmeyer, Carsten Konrad, Bernhard T Baune, Volker Arolt, Walter Heindel, Pienie Zwitserlood, and Thomas Suslow. Childhood maltreatment is associated with an automatic negative emotion processing bias in the amygdala. Human Brain Mapping, 000(February), June 2012a. ISSN 1097-0193. doi: 10.1002/hbm.22112. URL http://www.ncbi.nlm.nih.gov/pubmed/22696400.

Udo Dannlowski, Anja Stuhrmann, Victoria Beutelmann, Peter Zwanzger, Thomas Lenzen, Dominik Grotegerd, Katharina Domschke, Christa Hohoff, Patricia Ohrmann, Jochen 
Bauer, Christian Lindner, Christian Postert, Carsten Konrad, Volker Arolt, Walter Heindel, Thomas Suslow, and Harald Kugel. Limbic scars: long-term consequences of childhood maltreatment revealed by functional and structural magnetic resonance imaging. Biological Psychiatry, 71(4):286-93, February 2012b. ISSN 1873-2402. doi: 10.1016/j. biopsych.2011.10.021. URL http://www.ncbi.nlm.nih.gov/pubmed/22112927.

HN David. Towards a Reconceptualization of Striatal Interactions Between Glutamatergic and Dopaminergic Neurotransmission and Their Contribution to the Production. Current Neuropharmacology, 7(2):132-41, 2009. URL http://www.ncbi.nlm.nih.gov/pmc/ articles/PMC2730005/.

Neil Dawson, Brian J Morris, and Judith a Pratt. Subanaesthetic Ketamine Treatment Alters Prefrontal Cortex Connectivity With Thalamus and Ascending Subcortical Systems. Schizophrenia Bulletin, 6:1-12, November 2011. ISSN 1745-1701. doi: 10.1093/schbul/sbr144. URL http://www.ncbi.nlm.nih.gov/pubmed/22114100.

G. Deco and V. K. Jirsa. Ongoing Cortical Activity at Rest: Criticality, Multistability, and Ghost Attractors. Journal of Neuroscience, 32(10):3366-3375, March 2012. ISSN 0270-6474. doi: 10.1523/JNEUROSCI.2523-11.2012. URL http://ww .jneurosci.org/ cgi/doi/10.1523/JNEUROSCI.2523-11. 2012.

Gustavo Deco, Viktor K Jirsa, and Anthony R McIntosh. Emerging concepts for the dynamical organization of resting-state activity in the brain. Nature Reviews Neuroscience, 12(1):43-56, January 2011. ISSN 1471-0048. doi: 10.1038/nrn2961. URL http://wwb.ncbi.nlm.nih.gov/pubmed/21170073.

Ben Deen, Naomi B Pitskel, and Kevin a Pelphrey. Three systems of insular functional connectivity identified with cluster analysis. Cerebral Cortex, 21(7):1498-506, July 2011. ISSN 1460-2199. doi: 10.1093/cercor/bhq186. URL http://www pubmedcentral .nih. gov/articlerender. $f \mathrm{cgi}$ ? artid=3116731\&tool=pmcentrez\&rendertype=abstract.

A Del Arco and F Mora. Glutamate-dopamine in vivo interaction in the prefrontal cortex modulates the release of dopamine and acetylcholine in the nucleus accumbens of the awake rat. Journal of Neural Transmission, 112(1):97-109, January 2005. ISSN 03009564. doi: 10.1007/s00702-004-0172-5. URL http://www.ncbi.nlm.nih.gov/pubmed/ 
15599608 .

Alberto Del Arco and Francisco Mora. Neurotransmitters and prefrontal cortex-limbic system interactions: implications for plasticity and psychiatric disorders. Journal of Neural Transmission, 116(8):941-52, August 2009. ISSN 1435-1463. doi: 10.1007/ s00702-009-0243-8. URL http://www.springerlink.com/content/k21807181u854677/ http://www.ncbi.nlm.nih.gov/pubmed/19475335.

A Delorme and S Makeig. EEGLAB: an open source toolbox for analysis of single-trial EEG dynamics including independent component analysis. Journal of Neuroscience Methods, 134(1):9-21, 2004. URL http://www.sciencedirect.com/science/article/pii/ S0165027003003479.

Arnaud Delorme, Terrence Sejnowski, and Scott Makeig. Enhanced detection of artifacts in EEG data using higher-order statistics and independent component analysis. NeuroImage, 34(4):1443-9, February 2007. ISSN 1053-8119. doi: 10.1016/j.neuroimage. 2006.11.004. URL http://www.pubmedcentral.nih.gov/articlerender.fcgi?artid= 2895624\&tool=pmcentrez\&rendertype=abstract.

Emily L Dennis, Neda Jahanshad, Katie L McMahon, Greig I de Zubicaray, Nicholas G Martin, Ian B Hickie, Arthur W Toga, Margaret J Wright, and Paul M Thompson. Development of Brain Structural Connectivity between Ages 12 and 30: A 4-Tesla Diffusion Imaging Study in 439 Adolescents and Adults. NeuroImage, September 2012. ISSN 1095-9572. doi: 10.1016/j.neuroimage.2012.09.004. URL http://ww.ncbi.nlm.nih. gov/pubmed/22982357.

Rahul S Desikan, Florent Ségonne, Bruce Fischl, Brian T Quinn, Bradford C Dickerson, Deborah Blacker, Randy L Buckner, Anders M Dale, R Paul Maguire, Bradley T Hyman, Marilyn S Albert, and Ronald J Killiany. An automated labeling system for subdividing the human cerebral cortex on MRI scans into gyral based regions of interest. NeuroImage, 31(3):968-80, July 2006. ISSN 1053-8119. URL http://wwt.ncbi.nlm.nih.gov/ pubmed $/ 16530430$.

Manus J Donahue, Jamie Near, Jakob U Blicher, and Peter Jezzard. Baseline GABA concentration and AMI response. NeuroImage, 53(2):392-8, November 2010. ISSN 
1095-9572. doi: 10.1016/j.neuroimage.2010.07.017. URL http://www.ncbi.nlm.nih . gov/pubmed/20633664.

Manus J Donahue, Hans Hoogduin, Stephen M Smith, Jeroen C W Siero, Michael Chappell, Natalia Petridou, Peter Jezzard, Peter R Luijten, and Jeroen Hendrikse. Spontaneous blood oxygenation level-dependent fMRI signal is modulated by behavioral state and correlates with evoked response in sensorimotor cortex: A 7.0-T fMRI study. Human Brain Mapping, 33(3):511-22, March 2011. ISSN 1097-0193. doi: 10.1002/hbm.21228. URL http://ww.ncbi.nlm.nih.gov/pubmed/21455940.

J K Douglass, L Wilkens, E Pantazelou, and F Moss. Noise enhancement of information transfer in crayfish mechanoreceptors by stochastic resonance. Nature, 365(6444):337-40, September 1993. ISSN 0028-0836. doi: 10.1038/365337a0. URL http://www.ncbi.nlm. nih.gov/pubmed/8377824.

WC Drevets, H Burton, TO Videen, AZ Snyder, JR Simpson, and ME Raichle. Blood flow changes in human somatosensory cortex during anticipated stimulation. Nature, 373(6511):249-52, 1995. URL http://www . nature.com/nature/journal/v373/n6511/ abs/373249a0.html.

Annie Duchesne, Marc M Dufresne, and Ron M Sullivan. Sex differences in corticolimbic dopamine and serotonin systems in the rat and the effect of postnatal handling. Progress in Neuropsychopharmacology \& Biological Psychiatry, 33(2):251-61, March 2009. ISSN 0278-5846. doi: 10.1016/j.pnpbp.2008.11.012. URL http://www.ncbi.nlm.nih.gov/ pubmed/19100810.

Niall W Duncan and Georg Northoff. Overview of potential procedural and participantrelated confounds for neuroimaging of the resting state. Journal of Psychiatry $\&$ Neuroscience, 2012.

Niall W Duncan, Björn Enzi, Christine Wiebking, and Georg Northoff. Involvement of glutamate in rest-stimulus interaction between perigenual and supragenual anterior cingulate cortex: A combined fMRI-MRS study. Human Brain Mapping, 32(12): 2172-82, February 2011. ISSN 1097-0193, doi; 10.1002/hbm.21179. URL http: //ww.ncbi.nlm.nih.gov/pubmed/21305662. 
VJ Edwards, GW Holden, VJ Felitti, and RF Anda. Relationship between multiple forms of childhood maltreatment and adult mental health in community respondents: results from the adverse childhood experiences study. American Journal of Psychiatry, 160(8):145360, 2003. URL http://psychiatryonline.org/article.aspx?articleid=176375.

Björn Enzi, Niall W NW Duncan, Jörn Kaufmann, Claus Tempelmann, Christine Wiebking, and Georg Northoff. Glutamate Modulates Resting State Activity in the Perigenual Anterior Cingulate Cortex - A combined fMRI-MRS study. Neuroscience, 227:102-9, 2012. URL http://www.sciencedirect.com/science/article/pii/S0306452212009591.

S H Eriksson, S L Free, M Thom, M R Symms, L Martinian, J S Duncan, and S M Sisodiya. Quantitative grey matter histological measures do not correlate with grey matter probability values from in vivo MRI in the temporal lobe. Journal of Neuroscience Methods, 181(1):111-8, June 2009. ISSN 1872-678X. doi: 10.1016/j.jneumeth. 2009.05.001. URL http://www.pubmedcentral .nih.gov/articlerender.fcgi?artid= $2706955 \&$ tool=pmcentrez\&rendert ype=abstract .

A Etkin and Tor D Wager. Functional neuroimaging of anxiety: a meta-analysis of emotional processing in PTSD, social anxiety disorder, and specific phobia. American Journal of Psychiatry, 164(10):1476-1488, 2007.

Yan Fan, Niall W Duncan, Moritz de Greck, and Georg Northoff. Is there a core neural network in empathy? An fMRI based quantitative meta-analysis. Neuroscience and Biobehavioral Reviews, 35(3):903-11, January 2011. ISSN 1873-7528. doi: 10.1016/j. neubiorev.2010.10.009. URL http://www.ncbi.nlm.nih.gov/pubmed/20974173.

Norman S Farb, Zindel V Segal, and Adam K Anderson. Attentional modulation of primary interoceptive and exteroceptive cortices. Cerebral Cortex, pages 1-13, January 2012. ISSN 1460-2199. doi: 10.1093/cercor/bhr385. URL http://ww.ncbi.nlm.nih.gov/pubmed/ 22267308 .

Marco Fedi, Samuel F Berkovic, Carla Marini, Rachel Mulligan, Henri Tochon-Danguy, and David C Reutens. A GABAA receptor mutation causing generalized epilepsy reduces benzodiazepine receptor binding. NeuroImage, 32(3):995-1000, September 2006. ISSN 1053-8119. doi: 10.1016/j.neuroimage.2006.05.059. URL http://www.ncbi.nlm.nih. 
gov/pubmed/16875845.

M G P Feenstra, M H a Botterblom, and J F M van Uum. Behavioral arousal and increased dopamine efflux after blockade of NMDA-receptors in the prefrontal cortex are dependent on activation of glutamatergic neurotransmission. Neuropharmacology, 42(6):752-63, May 2002. ISSN 0028-3908. URL http://www.ncbi.nlm.nih.gov/pubmed/12015201.

Tommaso Fellin, Olivier Pascual, Sara Gobbo, Tullio Pozzan, Philip G Haydon, and Giorgio Carmignoto. Neuronal synchrony mediated by astrocytic glutamate through activation of extrasynaptic NMDA receptors. Neuron, 43(5):729-43, September 2004. ISSN 08966273. doi: 10.1016/j.neuron.2004.08.011. URL http://www.ncbi.nlm.nih.gov/pubmed/ 15339653.

Luiz K Ferreira, Breno S Diniz, Orestes V Forlenza, Geraldo F Busatto, and Marcus V Zanetti. Neurostructural predictors of Alzheimer's disease: a meta-analysis of VBM studies. Neurobiology of Aging, 32(10):1733-41, October 2011. ISSN 1558-1497, doi: 10.1016/ j.neurobiolaging.2009.11.008. URL http://ww. ncbi.nlm.nih.gov/pubmed/20005012.

Bruce Fischl, David H Salat, Evelina Busa, Marilyn Albert, Megan Dieterich, Christian Haselgrove, Andre van der Kouwe, Ron Killiany, David Kennedy, Shuna Klaveness, Albert Montillo, Nikos Makris, Bruce Rosen, and Anders M Dale. Whole brain segmentation: automated labeling of neuroanatomical structures in the human brain. Neuron, 33(3): 341-55, January 2002. ISSN 0896-6273. URL http://www.ncbi.nlm.nih.gov/pubmed/ 11832223.

Bruce Fischl, André van der Kouwe, Christophe Destrieux, Eric Halgren, Florent Ségonne, David H Salat, Evelina Busa, Larry J Seidman, Jill Goldstein, David Kennedy, Verne Caviness, Nikos Makris, Bruce Rosen, and Anders M Dale. Automatically parcellating the human cerebral cortex. Cerebral Cortex, 14(1):11-22, January 2004. ISSN 1047-3211. URL http://www.ncbi.nlm.nih.gov/pubmed/14654453.

Stan B Floresco, Ying Zhang, and Takeshi Enomoto. Neural circuits subserving behavioral flexibility and their relevance to schizophrenia. Behavioural Brain Research, 204(2):396409, December 2009. ISSN 1872-7549. doi: 10.1016/j.bbr.2008.12.001. URL http: //www.ncbi.nlm.nih.gov/pubmed/19110006. 
M.D. Fox, A.Z. Snyder, J.L. Vincent, M. Corbetta, D.C. Van Essen, and M.E. Raichle. The human brain is intrinsically organized into dynamic, anticorrelated functional networks. Proceedings of the National Academy of Sciences of the United States of America, 102 (27):9673, 2005. LRL http://www .pnas.org/content/102/27/9673. short.

Michael D Fox and Marcus E Raichle. Spontaneous fluctuations in brain activity observed with functional magnetic resonance imaging. Nature Reviews Neuroscience, 8(9):700-11, September 2007. ISSN 1471-003X. doi: 10.1038/nrn2201. URL http://www.ncbi.nlm. nih.gov/pubmed/17704812.

M Fraboni and D Cooper. Further validation of three short forms of the Marlowe-Crowne Scale of Social Desirability. Psychological Reports, 65:595-600, 1989.

V Francati, E Vermetten, and J D Bremner. Functional neuroimaging studies in posttraumatic stress disorder: review of current methods and findings. Depression and Anxiety, 24(3):202-18, January 2007. ISSN 1091-4269. doi: 10.1002/ da.20208. URL http://ww. pubmedcentral.nih.gov/articlerender.fcgi?artid= 3233765\&tool=pmcentrez\&rendertype=abstract.

W Gordon Frankle, Raymond Y Cho, N Scott Mason, Chi-Min Chen, Michael Himes, Christopher Walker, David a Lewis, Chester a Mathis, and Rajesh Narendran. [C]flumazenil binding Is increased in a dose-dependent manner with tiagabine-induced elevations in GABA levels. PloS One, 7(2):e32443, January 2012. ISSN 19326203. doi: 10.1371/journal.pone.0032443. URL http://ww. pubmedcentral.nih.gov/ articlerender.fcgi?artid=3288104\&tool=pmcentrez\&rendertype=abstract.

K J Friston, A P Holmes, J B Poline, P J Grasby, S C Williams, R S Frackowiak, and R Turner. Analysis of fMRI time-series revisited. NeuroImage, 2(1):45-53, March 1995. ISSN 1053-8119. URL http://www.ncbi.nlm.nih.gov/pubmed/9343589.

K J Friston, C Buechel, G R Fink, J Morris, E Rolls, and R J Dolan. Psychophysiological and modulatory interactions in neuroimaging. NeuroImage, 6(3):218-29, October 1997. ISSN 1053-8119. doi: 10.1006/nimg.1997.0291. URL http://ww.ncbi.nlm.nih.gov/ pubmed/9344826.

Cynthia H Y Fu, Kathryn M Abel, Matthew P G Allin, David Gasston, Sergi G Costafreda, 
John Suckling, Steve C R Williams, and Philip K McGuire. Effects of ketamine on prefrontal and striatal regions in an overt verbal fluency task: a functional magnetic resonance imaging study. Psychopharmacology, 183(1):92-102, November 2005. ISSN 00333158. doi: 10.1007/s00213-005-0154-9. URL http://ww.ncbi.nlm.nih.gov/pubmed/ 16228196 .

B M Gallagher, J S Fowler, N I Gutterson, R R MacGregor, C N Wan, and A P Wolf. Metabolic trapping as a principle of oradiopharmaceutical design: some factors resposible for the biodistribution of [18F] 2-deoxy-2-fluoro-D-glucose. Journal of Nuclear Medicine, 19(10):1154-61, October 1978. ISSN 0161-5505. URL http://ww.ncbi.nlm.nih.gov/ pubmed $/ 214528$.

Douglas D Garrett, Natasa Kovacevic, Anthony R McIntosh, and Cheryl L Grady. Blood oxygen level-dependent signal variability is more than just noise. The Journal of Neuroscience, 30(14):4914-21, April 2010. ISSN 1529-2401. doi: 10.1523/JNEUROSCI. 5166-09.2010. URL http://www.ncbi.nlm.nih.gov/pubmed/20371811.

Douglas D Garrett, Natasa Kovacevic, Anthony $R$ McIntosh, and Cheryl L Grady. The importance of being variable. The Journal of Neuroscience, 31(12):4496-503, March 2011. ISSN 1529-2401. doi: 10.1523/JNEUROSCI. 5641-10.2011. URL http://www.pubmedcentral.nih.gov/articlerender.fcgi? artid=3104038\&tool=pmcentrez\&rendertype=abstract.

Douglas D Garrett, Natasa Kovacevic, Anthony R McIntosh, and Cheryl L Grady. The modulation of $B O L D$ variability between cognitive states varies by age and processing speed. Cerebral Cortex, March 2012. ISSN 1460-2199. doi: 10.1093/cercor/bhs055. URL http://www.ncbi.nlm.nih.gov/pubmed/22419679.

Abigail G Garrity, Godfrey D Pearlson, Kristen McKiernan, Dan Lloyd, Kent A Kiehl, and Vince D Calhoun. Aberrant "default mode" functional connectivity in schizophrenia. The American Journal of Psychiatry, 164(3):450-7, March 2007. ISSN 0002-953X. doi: 10.1176/appi.ajp.164.3.450. URL http://www.ncbi.nlm.nih.gov/pubmed/17329470.

David N George, Trisha A Jenkins, and Simon Killcross. Dissociation of prefrontal cortex and nucleus accumbens dopaminergic systems in conditional learning in rats. Behavioural 
Brain Research, 225(1):47-55, June 2011. ISSN 1872-7549. doi: 10.1016/j.bbr.2011.06. 028. URL http://www.ncbi.nlm.nih.gov/pubmed/21741412.

Andrew Stuart Gibbons, Lucy Brooks, Elizabeth Scarr, and Brian Dean. AMPA receptor expression is increased post-mortem samples of the anterior cingulate from subjects with major depressive disorder. Journal of Affective Disorders, 136(3):1232-7, February 2012. ISSN 1573-2517, doi: 10.1016/j.jad.2011.10.001. URL http://ww .pubmedcentral.nih. gov/articlerender. fcgi ?artid=3275646\&tool=pmcentrez\&rendertype=abstract.

Rebecca Gittins and Paul J Harrison. A quantitative morphometric study of the human anterior cingulate cortex. Brain Research, 1013(2):212-22, July 2004. ISSN 0006-8993. doi: 10.1016/j.brainres.2004.03.064. URL http://ww .ncbi.nlm.nih.gov/ pubmed/15193531.

Yulia Golland, Shlomo Bentin, Hagar Gelbard, Yoav Benjamini, Ruth Heller, Yuval Nir, Uri Hasson, and Rafael Malach. Extrinsic and intrinsic systems in the posterior cortex of the human brain revealed during natural sensory stimulation. Cerebral Cortex, 17(4): 766-77, April 2007. ISSN 1047-3211. doi: 10.1093/cercor/bhk030. URL http://www. ncbi.nlm.nih.gov/pubmed/16699080.

Carlos Gómez, Roberto Hornero, Daniel Abásolo, Alberto Fernández, and Javier Escudero. Analysis of MEG background activity in Alzheimer's disease using nonlinear methods and ANFIS. Annals of Biomedical Engineering, 37(3):586-94, March 2009. ISSN 15216047. doi: 10.1007/s10439-008-9633-6. URL http://ww.ncbi.nlm.nih.gov/pubmed/ 19130227.

J. Gonzalez-Castillo, Z. S. Saad, D. a. Handwerker, S. J. Inati, N. Brenowitz, and P. a. Bandettini. Whole-brain, time-locked activation with simple tasks revealed using massive averaging and model-free analysis. Proceedings of the National Academy of Sciences, 109 (14):5487-5492, March 2012. ISSN 0027-8424. doi: 10.1073/pnas.1121049109. URL http://ww . pnas .org/cgi/doi/10.1073/pnas.1121049109.

F Gould, J Clarke, C Heim, PD Harvey, M Majer, and CB Nemeroff. The effects of child abuse and neglect on cognitive functioning in adulthood. Journal of Psychiatric Research, 46(4):500-506, 2012. URL http://www.sciencedirect.com/science/article/ 
$\mathrm{pii} / \mathrm{S} 0022395612000064$.

MM Grant, C Cannistraci, SD Hollon, J Gore, and R Shelton. Childhood trauma history differentiates amygdala response to sad faces within MDD. Journal of Psychiatric Research, 45(7):886-895, 2011. URL http://www.sciencedirect.com/science/article/ $\mathrm{pii} / \mathrm{S} 0022395610003456$.

Michael D Greicius, Ben Krasnow, Allan L Reiss, and Vinod Menon. Functional connectivity in the resting brain: a network analysis of the default mode hypothesis. Proceedings of the National Academy of Sciences of the United States of America, 100(1):253-8, January 2003. ISSN 0027-8424. doi: 10.1073/pnas. 0135058100. URL http://www.pubmedcentral.nih.gov/articlerender.fcgi?artid= $140943 \&$ tool=pmcentrez\&rendertype=abstract .

Michael D Greicius, Benjamin H Flores, Vinod Menon, Gary H Glover, Hugh B Solvason, Heather Kenna, Allan L Reiss, and Alan F Schatzberg. Resting-state functional connectivity in major depression: abnormally increased contributions from subgenual cingulate cortex and thalamus. Biological Psychiatry, 62(5):429-37, September 2007. ISSN 00063223. doi: 10.1016/j.biopsych.2006.09.020. URL http://www.pubmedcentral.nih.gov/ articlerender.fcgi? artid=2001244\&tool=pmcentrez\&render type=abstract.

Michael D Greicius, Kaustubh Supekar, Vinod Menon, and Robert F Dougherty. Resting-state functional connectivity reflects structural connectivity in the default mode network. Cerebral Cortex, 19(1):72-8, January 2009. ISSN 1460-2199. doi: 10.1093/cercor/bhn059. URL http://www.pubmedcentral.nih.gov/articlerender. fcgi?artid=2605172\&tool=pmcentrez\&rendertype=abstract.

Simone Grimm, Peter Boesiger, Johannes Beck, Daniel Schuepbach, Felix Bermpohl, Martin Walter, Jutta Ernst, Daniel Hell, Heinz Boeker, and Georg Northoff. Altered negative BOLD responses in the default-mode network during emotion processing in depressed subjects. Neuropsychopharmacology, 34(4):932-843, March 2009. ISSN 1740-634X. doi: 10.1038/npp.2008.81. URL http://www.ncbi.nlm.nih.gov/pubmed/18536699.

H J Groenewegen, C I Wright, A V Beijer, and P Voorn. Convergence and segregation of ventral striatal inputs and outputs. Annals of the New York Academy of Sciences, 
877:49-63, June 1999. ISSN 0077-8923. URL http://www.ncbi.nlm.nih.gov/pubmed/ 10415642 .

Aaron J Gruber, Rifat J Hussain, and Patricio O'Donnell. The nucleus accumbens: a switchboard for goal-directed behaviors. PloS One, 4(4):e5062, January 2009. ISSN 1932-6203. doi: 10.1371/journal.pone.0005062. URL http://ww .pubmedcentral.nih. gov/articlerender.fcgi ?artid $=2663037 \&$ tool=pmcentrez\&rendertype=abstract.

R Gruetter and I Tkác. Field mapping without reference scan using asymmetric echoplanar techniques. Magnetic Resonance in Medicine, 43(2):319-23, February 2000. ISSN 0740-3194. URL http://www.ncbi.nlm.nih.gov/pubmed/10680699.

X. Gu, Z. Gao, X. Wang, X. Liu, R. T. Knight, P. R. Hof, and J. Fan. Anterior insular cortex is necessary for empathetic pain perception. Brain, 135(9):2726-2735, September 2012. ISSN 0006-8950. doi: 10.1093/brain/aws199. URL http://www.ncbi.nlm.nih. gov/pubmed/22961548.

Roger Guimerà, Marta Sales-Pardo, and Luís A N Amaral. Classes of complex networks defined by role-to-role connectivity profiles. Nature Physics, 3(1):63-69, January 2007. ISSN 1745-2473. doi: 10.1038/nphys489. URL http://www.pubmedcentral.nih.gov/ articlerender.fcgi? artid=2447920\&tool=pmcentrez\&rendertype=abstract.

Eren Gultepe and Bin He. A linear/nonlinear characterization of resting state brain networks in fMRI time series. Brain Topography, September 2012. ISSN 1573-6792. doi: 10.1007/s10548-012-0249-7. URL http://www.ncbi.nlm.nih.gov/pubmed/22941499.

Debra A Gusnard and Marcus E Raichle. Searching for a baseline: Functional imaging and the human brain. Nature Reviews Neuroscience, 2(10):685-94, 2001.

M Gutièrrez-Mecinas, AF Trollope, A Collins, H Morfett, SA Hesketh, F Kersanté, and JM Reul. Long-lasting behavioral responses to stress involve a direct interaction of glucocorticoid receptors with ERK1/2-MSK1-Elk-1 signaling. Proceedings of the National Academy of Sciences of the United States of America, 108(33):13806-11, 2011. URL http://www . pnas.org/content/108/33/13806. short.

George Hadjipavlou, Paul Dunckley, Timothy E Behrens, and Irene Tracey. Deter- 
mining anatomical connectivities between cortical and brainstem pain processing regions in humans: a diffusion tensor imaging study in healthy controls. Pain, 123 (1-2):169-78, July 2006. ISSN 1872-6623. doi: 10.1016/j.pain.2006.02.027. URL http://www.ncbi.nlm.nih.gov/pubmed/16616418.

Patric Hagmann, Leila Cammoun, Xavier Gigandet, Reto Meuli, Christopher J Honey, Van J Wedeen, and Olaf Sporns. Mapping the structural core of human cerebral cortex. PLoS Biology, 6(7):e159, July 2008. ISSN 1545-7885. doi: 10.1371/journal.pbio. 0060159. URL http://www.pubmedcentral.nih.gov/articlerender.fcgi?artid= 2443193\&tool=pmcentrez\&rendert ype=abstract.

A Hammers, M J Koepp, M P Richardson, C Labbé, D J Brooks, V J Cunningham, and J S Duncan. Central benzodiazepine receptors in malformations of cortical development: A quantitative study. Brain, 124:1555-65, August 2001. ISSN 0006-8950. URL http: //ww.ncbi.nlm.nih.gov/pubmed/11459747.

A. Hammers, M.J. Koepp, M.P. Richardson, R. Hurlemann, D.J. Brooks, and J.S. Duncan. Grey and white matter flumazenil binding in neocortical epilepsy with normal MRI. A PET study of 44 patients. Brain, 126(6):1300, 2003. URL http://brain. oxfordjournals.org/content/126/6/1300. short.

Michelle Hampson, Naomi Driesen, Jennifer K Roth, John C Gore, and R Todd Constable. Functional connectivity between task-positive and task-negative brain areas and its relation to working memory performance. Magnetic Resonance Imaging, 28(8):1051-7, October 2010. ISSN 1873-5894. doi: 10.1016/j.mri. 2010.03.021. URL http://ww . pubmedcentral .nih.gov/articlerender.fcgi?artid= 2936669\&tool=pmcentrez\&rendert ype=abstract.

Susan M Hanson and Cynthia Czajkowski. Structural mechanisms underlying benzodiazepine modulation of the GABA(A) receptor. The Journal of Neuroscience, 28(13):3490-9, March 2008. ISSN 1529-2401. doi: 10.1523/JNEUROSCI. 5727-07.2008. URL http://www.pubmedcentral.nih.gov/articlerender.fcgi? artid $=2410040 \&$ tool=pmcentrez\&rendertype=abstract .

Xuejun Hao, Dongrong Xu, Ravi Bansal, Zhengchao Dong, Jun Liu, Zhishun Wang, Alayar 
Kangarlu, Feng Liu, Yunsuo Duan, Satie Shova, Andrew J. Gerber, and Bradley S. Peterson. Multimodal magnetic resonance imaging: The coordinated use of multiple, mutually informative probes to understand brain structure and function. Human Brain Mapping, November 2011. ISSN 10659471. doi: 10.1002/hbm.21440. URL http://doi . wiley.com/10.1002/hbm. 21440 .

Heledd Hart and Katya Rubia. Neuroimaging of child abuse: a critical review. Frontiers in Human Neuroscience, 6:1-24, 2012. ISSN 1662-5161. doi: 10.3389/fnhum.2012. 00052. URL http://ww.frontiersin.org/Human_Neuroscience/10.3389/fnhum. $2012.00052 /$ abstract.

Brian H Harvey and Mohammed Shahid. Metabotropic and ionotropic glutamate receptors as neurobiological targets in anxiety and stress-related disorders: focus on pharmacology and preclinical translational models. Pharmacology, Biochemistry, and Behavior, 100 (4):775-800, February 2012. ISSN 1873-5177. doi: 10.1016/j.pbb.2011.06.014. URL http://ww. ncbi.nlm.nih.gov/pubmed/21708184.

Kenji Hashimoto. Emerging role of glutamate in the pathophysiology of major depressive disorder. Brain Research Reviews, 61(2):105-23, October 2009. ISSN 1872-6321. doi: 10.1016/j.brainresrev.2009.05.005. URL http://www.ncbi.nlm.nih.gov/pubmed/ 19481572.

Gregor Hasler, Jan Willem van der Veen, Toni Tumonis, Noah Meyers, Jun Shen, and Wayne C Drevets. Reduced prefrontal glutamate/glutamine and gamma-aminobutyric acid levels in major depression determined using proton magnetic resonance spectroscopy. Archives of General Psychiatry, 64(2):193 200, February 2007. ISSN 0003-990X. doi: 10.1001/archpsyc.64.2.193. URL http://ww.ncbi.nlm.nih.gov/pubmed/17283286.

Erik R Hauge, Jan $\emptyset$ ystein Berle, Ketil J Oedegaard, Fred Holsten, and Ole Bernt Fasmer. Nonlinear analysis of motor activity shows differences between schizophrenia and depression: a study using Fourier analysis and sample entropy. PloS One, 6(1):e16291, January 2011. ISSN 1932-6203. doi: 10.1371/journal.pone. 0016291. URL http://ww.pubmedcentral.nih.gov/articlerender.fcgi?artid= 3030566\&tool=pmcentrez\&rendertype=abstract. 
Dave J. Hayes and Georg Northoff. Identifying a network of brain regions involved in aversion-related processing: A cross-species translational investigation. Frontiers in Integrative Neuroscience, 5(October):1-21, 2011. ISSN 1662-5145. doi: 10.3389/ fnint.2011.00049. URL http://www.frontiersin.org/Integrative_Neuroscience/ 10.3389/fnint. $2011.00049 /$ abstract.

Dave $\mathrm{J}$ Hayes and Georg Northoff. Common brain activations for painful and nonpainful aversive stimuli. BMC Neuroscience, 13(1):60, June 2012. ISSN 1471-2202. doi: 10.1186/ 1471-2202-13-60. URL http://www.ncbi.nlm.nih.gov/pubmed/22676259.

Yong He, Zhang J Chen, and Alan C Evans. Small-world anatomical networks in the human brain revealed by cortical thickness from MRI. Cerebral Cortex, 17(10):2407-19, October 2007. ISSN 1047-3211. doi: 10.1093/cercor/bhl149. URL http://www.ncbi.nlm.nih. gov/pubmed/17204824.

Lizette Heine, Andrea Soddu, Francisco Gómez, Audrey Vanhaudenhuyse, Luaba Tshibanda, Marie Thonnard, Vanessa Charland-Verville, Murielle Kirsch, Steven Laureys, and Athena Demertzi. Resting state networks and consciousness. Frontiers in Psychology, 3(August):1-12, 2012. ISSN 1664-1078. doi: 10.3389/fpsyg.2012.00295. URL http://www frontiersin.org/Cognition/10.3389/fpsyg.2012.00295/abstract.

W. D. Heiss, M. Grond, A. Thiel, M. Ghaemi, J. Sobesky, J. Rudolf, B. Bauer, and K. Wienhard. Permanent cortical damage detected by flumazenil positron emission tomography in acute stroke. Stroke, 29(2):454-461, February 1998. ISSN 0039-2499. doi: 10.1161/01.STR.29.2.454. URL http://stroke.ahajournals.org/cgi/doi/10.1161/ 01.STR.29.2.454.

Wolf-Dieter Heiss and Karl Herholz. Brain receptor imaging. Journal of Nuclear Medicine, 47(2):302-12, February 2006. ISSN 0161-5505. URL http://www.ncbi.nlm.nih.gov/ pubmed/16455637.

Stephen G Henry. Polanyi's tacit knowing and the relevance of epistemology to clinical medicine. Journal of Evaluation in Clinical Practice, 16(2):292-297, 2010. URL http: //ww.ncbi.nlm.nih.gov/pubmed/20367850.

Guido Hesselmann, Christian A Kell, Evelyn Eger, and Andreas Kleinschmidt. Spon- 
taneous local variations in ongoing neural activity bias perceptual decisions. Proceedings of the National Academy of Sciences of the United States of America, 105(31):10984-9, August 2008. ISSN 1091-6490. doi: 10.1073/pnas. 0712043105. URL http://ww. pubmedcentral.nih.gov/articlerender.fcgi?artid= 2504783\&tool=pmcentrez\&rendertype=abstract.

Guido Hesselmann, Sepideh Sadaghiani, Karl J Friston, and Andreas Kleinschmidt. Predictive coding or evidence accumulation? False inference and neuronal fluctuations. PloS one, 5(3):e9926, January 2010. ISSN 1932-6203. doi: 10.1371/journal.pone. 0009926. URL http://www.pubmedcentral.nih.gov/articlerender.fcgi?artid= 2848028\&tool=pmcentrez\&rendertype=abstract.

JM Hettema, B Kettenmann, V Ahluwalia, C McCarthy, WR Kates, JE Schmitt, JL Silberg, MC Neale, KS Kendler, and P Fatouros. Pilot multimodal twin imaging study of generalized anxiety disorder. Depression and Anxiety, 29(3), 2011. URL http: //onlinelibrary.wiley.com/doi/10.1002/da.20901/full.

F Hofheinz, J Langner, B Beuthien-Baumann, L Oehme, J Steinbach, J Kotzerke, and $\mathrm{J}$ van der Hoff. Suitability of bilateral filtering for edge-preserving noise reduction in PET. EJNMMI Research, 1(23), 2011. URL http://ww.springerlink.com/index/ 32041633U7601564.pdf.

C J Honey, O Sporns, L Cammoun, X Gigandet, J P Thiran, R Meuli, and P Hagmann. Predicting human resting-state functional connectivity from structural connectivity. Proceedings of the National Academy of Sciences of the United States of America, 106(6):2035-40, February 2009. ISSN 1091-6490. doi: 10.1073/pnas. 0811168106. URL http://www .pubmedcentral .nih.gov/articlerender.fcgi?artid= $2634800 \&$ tool=pmcentrez\&rendertype=abstract .

Garry D Honey, Philip R Corlett, Anthony R Absalom, Michael Lee, Edith Pomarol-Clotet, Graham K Murray, Peter J McKenna, Edward T Bullmore, David K Menon, and Paul C Fletcher. Individual differences in psychotic effects of ketamine are predicted by brain function measured under placebo. The Journal of Neuroscience, 28(25):6295-303, June 2008. ISSN 1529-2401. doi: 10.1523/JNEUROSCI.0910-08.2008. URL http://www . ncbi.nlm.nih.gov/pubmed/18562599. 
IK Hong, ST Chung, HK Kim, and YB Kim. Lltra fast symmetry and SIMD-based projection-backprojection (SSP) algorithm for 3-D PET image reconstruction. IEEE Trans Med Imaging, 26:789-803, 2007. doi: 10.1109/TMI.2002.808360. LRL http: //ieeexplore.ieee.org/xpls/abs_all.jsp?arnumber $=4214878$.

Jill M Hooley, Staci a Gruber, Holly a Parker, Julien Guillaumot, Jadwiga Rogowska, and Deborah a Yurgelun-Todd. Cortico-limbic response to personally challenging emotional stimuli after complete recovery from depression. Psychiatry Research, 171 (2):106-119, February 2009. ISSN 0165-1781. doi: 10.1016/j.pscychresns.2008.04. 001. URL http://ww.sciencedirect.com/science/article/pii/s0925-4927(08) 00057-7http://www.ncbi.nlm.nih.gov/pubmed/19176279.

Dorothea I Horn, Chunshui Yu, Johann Steiner, Julia Buchmann, Joern Kaufmann, Annemarie Osoba, Ulf Eckert, Kathrin C Zierhut, Kolja Schiltz, Huiguang He, Bharat Biswal, Bernhard Bogerts, and Martin Walter. Glutamatergic and restingstate functional connectivity correlates of severity in major depression - the role of pregenual anterior cingulate cortex and anterior insula. Frontiers in Systems Neuroscience, 4(July):1-10, January 2010. ISSN 1662-5137. doi: 10.3389/fnsys. 2010.00033. URL http://ww.pubmedcentral.nih.gov/articlerender.fcgi?artid= 2914530\&tool=pmcentrez\&rendertype=abstract.

H.M. Hudson and R.S. Larkin. Accelerated image reconstruction using ordered subsets of projection data. IEEE Transactions on Medical Imaging, 13(4):601-609, 1994. URL http://ieeexplore. ieee.org/xpls/abs_all . jsp?arnumber $=363108$.

K Hüfner, T Stephan, S Glasauer, R Kalla, E Riedel, A Deutschländer, T Dera, M Wiesmann, M Strupp, and T Brandt. Differences in saccade-evoked brain activation patterns with eyes open or eyes closed in complete darkness. Experimental Brain Research, 186(3):419-30, April 2008. ISSN 1432-1106. doi: 10.1007/s00221-007-1247-y. URL http://www.ncbi.nlm.nih.gov/pubmed/18183378.

K Hüfner, T Stephan, V L Flanagin, A Deutschländer, A Stein, R Kalla, T Dera, G Fesl, $\mathrm{K}$ Jahn, M Strupp, and T Brandt. Differential effects of eyes open or closed in darkness on brain activation patterns in blind subjects. Neuroscience Letters, 466(1):30-4, November 2009. ISSN 1872-7972. doi: 10.1016/j.neulet.2009.09.010. URL http://www.ncbi.nlm. 
nih.gov/pubmed/19766168.

David Hume. An enquiry concerning human understanding. Oxford University Press, Oxford, UK, 1748. URL http://books.google.com/books?hl= en\&lr $=\& i d=R 6 d 35$ eiOKoC\&Oi $=$ fnd\&pg=PR9\&dq=An+Enquiry+Concerning +Human+ Understanding\&ots $=b-7 b E T 7 Z c D \& s i g=N S z n 2 A o Q N R 4 L$ fddtrNiE85tNfEU.

M D Humphries, $\mathrm{K}$ Gurney, and $\mathrm{T} \mathrm{J}$ Prescott. The brainstem reticular formation is a small-world, not scale-free, network. Proceedings. Biological sciences / The Royal Society, 273(1585):503-11, February 2006. ISSN 0962-8452. doi: 10.1098/rspb. 2005.3354. URL http://ww . pubmedcentral.nih.gov/articlerender.fcgi?artid= 1560205\&tool=pmcentrez\&rendertype=abstract.

W Hunkeler, H Möhler, L Pieri, P Polc, E P Bonetti, R Cumin, R Schaffner, and W Haefely. Selective antagonists of benzodiazepines. Nature, 290(5806):514-6, April 1981. ISSN 0028-0836. URL http://www.ncbi.nlm.nih.gov/pubmed/6261143.

$\mathrm{R}$ Matthew Hutchison and Stefan Everling. Monkey in the middle: why nonhuman primates are needed to bridge the gap in resting-state investigations. Frontiers in Neuroanatomy, 6:29, January 2012. ISSN 1662-5129. doi: 10.3389/fnana. 2012.00029. URL http://www pubmedcentral .nih.gov/articlerender.fcgi?artid= $3405297 \&$ tool=pmcentrez\&render type=abstract.

Robert B Innis, Vincent J Cunningham, Jacques Delforge, Masahiro Fujita, Albert Gjedde, Roger N Gunn, James Holden, Sylvain Houle, Sung-Cheng Huang, Masanori Ichise, Hidehiro Iida, Hiroshi Ito, Yuichi Kimura, Robert a Koeppe, Gitte M Knudsen, Juhani Knuuti, Adriaan a Lammertsma, Marc Laruelle, Jean Logan, Ralph Paul Maguire, Mark a Mintun, Evan D Morris, Ramin Parsey, Julie C Price, Mark Slifstein, Vesna Sossi, Tetsuya Suhara, John R Votaw, Dean F Wong, and Richard E Carson. Consensus nomenclature for in vivo imaging of reversibly binding radioligands. Journal of Cerebral Blood Flow and Metabolism, 27(9):1533-9, September 2007. ISSN 0271-678X. doi: 10.1038/sj.jcbfm.9600493. URL http://www.ncbi.nlm.nih.gov/pubmed/17519979.

AP Jackowski, H Douglas-Palumberi, M Jackowski, L Win, RT Schultz, LW Staib, JH Krystal, and J Kaufman. Corpus callosum in maltreated children with posttraumatic stress 
disorder: a diffusion tensor imaging study. Psychiatry Research, 162(3):256-61, 2008. LRL http://www.sciencedirect.com/science/article/pii/S0925492707001564.

Mark E Jackson, Houman Homayoun, and Bita Moghaddam. N.MDA receptor hypofunction produces concomitant firing rate potentiation and burst activity reduction in the prefrontal cortex. Proceedings of the National Academy of Sciences of the United States of America, 101(22):8467-72, June 2004. ISSN 0027-8424. doi: 10.1073/pnas. 0308455101. URL http://ww. pubmedcentral.nih.gov/articlerender.fcgi?artid= $420417 \&$ tool=pmcentrez\&rendertype=abstract.

Tija C Jacob, Stephen J Moss, and Rachel Jurd. GABA(A) receptor trafficking and its role in the dynamic modulation of neuronal inhibition. Nature Reviews Neuroscience, 9(5):331-43, May 2008. ISSN 1471-0048. doi: 10.1038/ nm2370. URL http://www.pubmedcentral.nih.gov/articlerender.fcgi?artid= 2709246\&tool=pmcentrez\&rendertype=abstract.

Shlomit Jacobson-Pick and Gal Richter-Levin. Short- and long-term effects of juvenile stressor exposure on the expression of GABAA receptor subunits in rats. Stress, 15(4): 416-24, July 2012. ISSN 1607-8888. doi: 10.3109/10253890.2011.634036. URL http: //www.ncbi.nlm.nih.gov/pubmed/22044189.

Sajjida Jaffer, Vasily Vorobyov, Peter C Kind, and Frank Sengpiel. Experience-dependent regulation of functional maps and synaptic protein expression in the cat visual cortex. The European Journal of Neuroscience, 35(8):1281-94, April 2012. ISSN 1460-9568. doi: 10.1111/j.1460-9568.2012.08044.x. URL http://ww.ncbi.nlm.nih.gov/pubmed/ 22512257.

T. A. Jarrell, Y. Wang, A. E. Bloniarz, C. A. Brittin, M. Xu, J. N. Thomson, D. G. Albertson, D. H. Hall, and S. W. Emmons. The Connectome of a Decision-Making Neural Network. Science, 337(6093):437-444, July 2012. ISSN 0036-8075. doi: 10.1126/ science.1221762. URL http://www.sciencemag.org.proxy.bib.uottawa.ca/content/ 337/6093/437. full.

Norbert Jausovec and Ksenija Jausovec. Resting brain activity: differences between genders. Neuropsychologia, 48(13):3918-25, November 2010. ISSN 1873-3514. doi: 10.1016/j. 
neuropsychologia.2010.09.020. LRL http://www.ncbi.nlm.nih.gov/pubmed/20875436.

E Jodo, T Katayama, M Okamoto, Y Suzuki, K Hoshino, and Y Kayama. Differences in responsiveness of mediodorsal thalamic and medial prefrontal cortical neurons to social interaction and systemically administered phencyclidine in rats. Neuroscience, 170(4): 1153-64, November 2010. ISSN 1873-7544. doi: 10.1016/j.neuroscience.2010.08.017. URL http://ww.ncbi.nlm.nih.gov/pubmed/20727386.

Eiichi Jodo, Yoshiaki Suzuki, Tadahiro Katayama, Ken-Yo Hoshino, Satoshi Takeuchi, ShinIchi Niwa, and Yukihiko Kayama. Activation of medial prefrontal cortex by phencyclidine is mediated via a hippocampo-prefrontal pathway. Cerebral Cortex, 15(5):663-9, May 2005. ISSN 1047-3211. doi: 10.1093/cercor/bhh168. URL http://www.ncbi.nIm.nih. gov/pubmed/15342431.

Heidi Johansen-Berg, Timothy E J Behrens, Emma Sillery, Olga Ciccarelli, Alan J Thompson, Stephen M Smith, and Paul M Matthews. Functional-anatomical validation and individual variation of diffusion tractography-based segmentation of the human thalamus. Cerebral Cortex, 15(1):31-9, January 2005. ISSN 1047-3211. doi: 10.1093/cercor/bhh105. URL http://www.ncbi.nlm.nih.gov/pubmed/15238447.

Dietsje D. Jolles, Mark a. van Buchem, Eveline a. Crone, and Serge a.R.B. Rombouts. Functional brain connectivity at rest changes after working memory training. Human Brain Mapping, November 2011. ISSN 10659471. doi: 10.1002/hbm.21444. URL http: $/ /$ doi.wiley.com/10.1002/hbm. 21444 .

Edward G Jones. Synchrony in the interconnected circuitry of the thalamus and cerebral cortex. Annals of the New York Academy of Sciences, 1157:10-23, March 2009. ISSN 1749-6632. doi: 10.1111/j.1749-6632.2009.04534.x. URL http://ww.ncbi.nlm.nih. gov/pubmed/19351352.

C Judo, M Matsumoto, D Yamazaki, and S Hiraide. Early stress exposure impairs synaptic potentiation in the rat medial prefrontal cortex underlying contextual fear extinction. Neuroscience, 169(4):1705-14, 2010. URL http://www.sciencedirect.com/science/ article/pii/S0306452210008821.

C Juhász, O Muzik, D C Chugani, C Shen, J Janisse, and H T Chugani. Prolonged 
vigabatrin treatment modifies developmental changes of GABA(A)-receptor binding in young children with epilepsy. Epilepsia, 42(10):1320-6, October 2001. ISSN 0013-9580. URL http://www.ncbi.nlm.nih.gov/pubmed/11737167.

Csaba Juhász, Eishi Asano, Aashit Shah, Diane C Chugani, Carlos E A Batista, Otto Muzik, Sandeep Sood, and Harry $T$ Chugani. Focal decreases of cortical GABAA receptor binding remote from the primary seizure focus: what do they indicate? Epilepsia, 50(2):240-50, February 2009. ISSN 1528-1167. doi: 10.1111/j. 1528-1167.2008.01721.x. URL http://www.pubmedcentral.nih.gov/articlerender. fcgi?artid=2642902\&tool=pmcentrez\&rendertype=abstract .

R. Kanai, B. Bahrami, R. Roylance, and G. Rees. Online social network size is reflected in human brain structure. Proceedings of the Royal Society, October 2011. ISSN 0962-8452. doi: 10.1098/rspb.2011.1959. URL http://www.ncbi.nlm.nih.gov/pubmed/22012980.

Ryota Kanai and Geraint Rees. The structural basis of inter-individual differences in human behaviour and cognition. Nature Reviews Neuroscience, 12(4):231-42, April 2011. ISSN 1471-0048. doi: 10.1038/nrn3000. URL http://www.ncbi.nlm.nih.gov/pubmed/ 21407245 .

Dimitrios Kapogiannis, David a Reiter, Auriel a Willette, and Mark P Mattson. Posteromedial cortex glutamate and GABA predict intrinsic functional connectivity of the default mode network. NeuroImage, 64C:112-119, September 2012. ISSN 1095-9572. doi: 10.1016/j.neuroimage.2012.09.029. URL http://www.ncbi.nlm.nih.gov/pubmed/ 23000786 .

Lucila Kargieman, Noemí Santana, Guadalupe Mengod, Pau Celada, and Francesc Artigas. Antipsychotic drugs reverse the disruption in prefrontal cortex function produced by NMDA receptor blockade with phencyclidine. Proceedings of the National Academy of Sciences of the United States of America, 104(37):14843-8, September 2007. ISSN 0027-8424. doi: 10.1073/pnas.0704848104. URL http://www pubmedcentral.nih.gov/ articlerender. $\mathrm{f} c \mathrm{gi}$ ?artid=1976198\&tool=pmcentrez\&rendertype=abstract.

T Katayama, E Jodo, Y Suzuki, K-Y Hoshino, S Takeuchi, and Y Kayama. Activation of medial prefrontal cortex neurons by phencyclidine is mediated via AMPA/kainate 
glutamate receptors in anesthetized rats. Neuroscience, 150(2):442-8, December 2007. ISSN 0306-4522. doi: 10.1016/j.neuroscience.2007.09.007. URL http://www.ncbi.nlm. nih.gov/pubmed/17935894.

Robert E Kelly, George S Alexopoulos, Zhishun Wang, Faith M Gunning, Christopher F Murphy, Sarah Shizuko Morimoto, Dora Kanellopoulos, Zhiru Jia, Kelvin O Lim, and Matthew J Hoptman. Visual inspection of independent components: defining a procedure for artifact removal from fMRI data. Journal of Neuroscience Methods, 189(2):233-45, June 2010. ISSN 1872-678X. doi: 10.1016/j.jneumeth. 2010.03.028. URL http://www. pubmedcentral.nih.gov/articlerender.fcgi?artid= 3299198\&tool=pmcentrez\&rendert ype=abstract.

Jong-Hoon Kim, Sang Hoon Yi, Jinyoung Lee, and Yong Sik Kim. Effects of clozapine on heart rate dynamics and their relationship with therapeutic response in treatmentresistant schizophrenia. Journal of Clinical Psychopharmacology, 33(1):69-73, February 2013. ISSN 1533-712X. doi: 10.1097/JCP.0b013e31827d14e3. URL http://www.ncbi. nlm.nih.gov/pubmed/23277266.

Seong-Gi Kim and Seiji Ogawa. Biophysical and physiological origins of blood oxygenation level-dependent fMRI signals. Journal of Cerebral Blood Flow and Metabolism, 32(7): 1188-206, July 2012. ISSN 1559-7016. doi: 10.1038/jcbfm.2012.23. URL http://www . ncbi.nlm.nih.gov/pubmed/22395207.

a Kimura, H Imbe, and T Donishi. Efferent connections of an auditory area in the caudal insular cortex of the rat: anatomical nodes for cortical streams of auditory processing and cross-modal sensory interactions. Neuroscience, 166(4):1140-57, April 2010. ISSN 1873-7544. doi: 10.1016/j.neuroscience.2010.01.032. URL http://ww.ncbi.nlm.nih . gov/pubmed/20105453.

Shinobu Kitayama and Jiyoung Park. Cultural neuroscience of the self: understanding the social grounding of the brain. Social Cognitive and Affective Neuroscience, 5(2-3):111-29, June 2010. ISSN 1749-5024. doi: $10.1093 /$ scan/nsq052. URL http://scan . oxfordjournals.org.proxy .bib. uottawa.ca/content/5/2-3/111. full?maxtoshow=\&hits=10\&RESULTFORMAT =\&fulltext=Cultural+neuroscience of the+self: tunderstanding+the+social+grounding+of the+brain\&searchid= 
1\&FIRSTINDEX=0\&resourcetype=HWCIT.

W Klimesch. EEG alpha and theta oscillations reflect cognitive and memory performance: a review and analysis. Brain Research, 29(2-3):169-95, April 1999. URL http://www. ncbi.nlm.nih.gov/pubmed/10209231.

Ursula M H Klumpers, Dick J Veltman, Ronald Boellaard, Emile F Comans, Cassandra Zuketto, Maqsood Yaqub, Jurgen E M Mourik, Mark Lubberink, Witte J G Hoogendijk, and Adriaan a Lammertsma. Comparison of plasma input and reference tissue models for analysing $[(11) \mathrm{C} \mid$ flumazenil studies. Journal of Cerebral Blood Flow and Metabolism, 28(3):579-87, March 2008. ISSN 0271-678X. doi: 10.1038/sj.jcbfm.9600554. URL http: //www.ncbi.nlm.nih.gov/pubmed/17928801.

D Knox, SA Perrine, SA George, MP Galloway, and I Liberzon. Single prolonged stress decreases glutamate, glutamine, and creatine concentrations in the rat medial prefrontal cortex. Neuroscience, 480(1):16-20, 2010. URL http://www.sciencedirect.com/science/ article/pii/S0304394010006476.

B Knutson, a Westdorp, E Kaiser, and D Hommer. FMRI visualization of brain activity during a monetary incentive delay task. NeuroImage, 12(1):20-7, July 2000. ISSN 1053-8119. doi: 10.1006/nimg.2000.0593. URL http://www.ncbi.nlm.nih.gov/pubmed/10875899.

Hedy Kober, Lisa Feldman Barrett, Josh Joseph, Eliza Bliss-Moreau, Kristen Lindquist, and Tor D Wager. Functional grouping and cortical-subcortical interactions in emotion: a meta-analysis of neuroimaging studies. NeuroImage, 42(2):998-1031, August 2008. ISSN 1095-9572. doi: 10.1016/j.neuroimage. 2008.03.059. URL http://www.pubmedcentral .nih.gov/articlerender.fcgi?artid= $2752702 \&$ tool=pmcentrez\&rendert ype=abstract.

Jakub Z Konarski, Roger S McIntyre, Sidney H Kennedy, Shahryar Rafi-Tari, Joanna K Soczynska, and Terence A Ketter. Volumetric neuroimaging investigations in mood disorders: bipolar disorder versus major depressive disorder. Bipolar Disorders, 10(1): 1-37, February 2008. ISSN 1398-5647. doi: 10.1111/j.1399-5618.2008.00435.x. URL http://www.ncbi.nlm.nih.gov/pubmed/18199239.

Jian Kong, Pei-chi Tu, Carolyn Zyloney, and Tung-ping Su. Intrinsic functional 
connectivity of the periaqueductal gray, a resting fMRI study. Behavioural Brain Research, 211(2):215-9, August 2010. ISSN 1872-7549. doi: 10.1016/j.bbr. 2010.03.042. URL http://www.pubmedcentral.nih.gov/articlerender.fcgi?artid= 2862838\&tool=pmcentrez\&rendertype=abstract.

Simone Kühn and Jürgen Gallinat. Resting-State Brain Activity in Schizophrenia and Major Depression: A Quantitative Meta-Analysis. Schizophrenia Bulletin, pages 1-8, November 2011. ISSN 1745-1701. doi: 10.1093/schbul/sbr151. URL http://www .nebi. nlm.nih.gov/pubmed/22080493.

JR Kuo, PR Goldin, K Werner, and RG Heimberg. Childhood trauma and current psychological functioning in adults with social anxiety disorder. Journal of Anxiety Disorders, 25(4):467-73, 2011. URL http://www.sciencedirect.com/science/article/ $\mathrm{pii} / \mathrm{S} 088761851000229 \mathrm{X}$.

Christian la Fougère, Sarah Grant, Alexey Kostikov, Ralf Schirrmacher, Paul Gravel, Hyman M Schipper, Andrew Reader, Alan Evans, and Alexander Thiel. Where in-vivo imaging meets cytoarchitectonics: the relationship between cortical thickness and neuronal density measured with high-resolution [18F|flumazenil-PET. NeuroImage, 56(3): 951-60, June 2011. ISSN 1095-9572. doi: 10.1016/j.neuroimage.2010.11.015. URL http://www.ncbi.nlm.nih.gov/pubmed/21073964.

Jaakko W Lå ngsjö, Elina Salmi, Kaike K Kaisti, Sargo Aalto, Susanna Hinkka, Riku Aantaa, Vesa Oikonen, Tapio Viljanen, Timo Kurki, Martti Silvanto, and Harry Scheinin. Effects of subanesthetic ketamine on regional cerebral glucose metabolism in humans. Anesthesiology, 100(5):1065-71, May 2004. ISSN 0003-3022. URL http://www.ncbi. nlm.nih.gov/pubmed/15114201.

Alexandra Ladouceur, Jessica Tessier, Benjamin Provencher, Pierre Rainville, and Mathieu Piché. Top-down attentional modulation of analgesia induced by heterotopic noxious counterstimulation. Pain, 153(8):1755-1762, August 2012. ISSN 1872-6623. doi: 10. 1016/j.pain.2012.05.019. URL http://www.ncbi.nlm.nih.gov/pubmed/22717100.

Martin Lauritzen, Claus Mathiesen, Katharina Schaefer, and Kirsten J Thomsen. Neuronal inhibition and excitation, and the dichotomic control of brain hemodynamic and oxygen 
responses. NeuroImage, pages 1-11, January 2012. ISSN 1095-9572. doi: 10.1016/j. neuroimage.2012.01.040. URL http://ww.ncbi.nlm.nih.gov/pubmed/22261372.

C Lebel, L Walker, A Leemans, L Phillips, and C Beaulieu. Microstructural maturation of the human brain from childhood to adulthood. NeuroImage, 40(3):1044-55, April 2008. ISSN 1053-8119. doi: 10.1016/j.neuroimage.2007.12.053. URL http://ww.ncbi.nlm. nih.gov/pubmed/18295509.

S J-J Leistedt, P Linkowski, J-P Lanquart, J E Mietus, R B Davis, A L Goldberger, and M D Costa. Decreased neuroautonomic complexity in men during an acute major depressive episode: analysis of heart rate dynamics. Translational Psychiatry, 1:e27, January 2011. ISSN 2158-3188. doi: 10.1038/tp.2011.23. URL http://www.pubmedcentral.nih.gov/ articlerender . fcgi ?artid=3309515\&tool=pmcentrez\&rendertype=abstract .

Tyler A Lesh, Tara A Niendam, Michael J Minzenberg, and Cameron S Carter. Cognitive control deficits in schizophrenia: mechanisms and meaning. Neuropsychopharmacology, 36(1):316-38, January 2011. ISSN 1740-634X. doi: 10.1038/npp. 2010.156. URL http://www.pubmedcentral.nih.gov/articlerender.fcgi?artid= 3052853\& tool=pmcentrez\&rendert ype=abstract .

Yi Liao, Xiaoqi Huang, Qizhu Wu, Chuang Yang, Weihong Kuang, Mingying Du, Su Lui, Qiang Yue, Raymond C K Chan, Graham J Kemp, and Qiyong Gong. Is depression a disconnection syndrome? Meta-analysis of diffusion tensor imaging studies in patients with MDD. Journal of Psychiatry \& Neuroscience, 37(4):110180, June 2012. ISSN 1488-2434. doi: 10.1503/jpn.110180. URL http://www.ncbi.nlm.nih.gov/pubmed/22691300.

I Liberzon, A King, J Britton, and K Phan. Paralimbic and medial prefrontal cortical involvement in neuroendocrine responses to traumatic stimuli. American Journal of Psychiatry, 164(8):1250-8, 2007. URL http://neuro.psychiatryonline.org/article. aspx?articleid=98810\&amp; RelatedWidgetArticles=true.

Lia C Liefaard, Bart A Ploeger, Carla F M Molthoff, Hugo W A M de Jong, Jouke Dijkstra, Louise van der Weerd, Adriaan A Lammertsma, Meindert Danhof, and Rob A Voskuyl. Changes in GABAA receptor properties in amygdala kindled animals: in vivo studies using $[11 \mathrm{C}]$ flumazenil and positron emission tomography. Epilepsia, 50(1):88-98, January 
2009. ISSN 1528-1167. doi: 10.1111/j.1528-1167.2008.01763.x. URL http://www.ncbi. nlm.nih.gov/pubmed/18727682.

Kristen a Lindquist, Tor D Wager, Hedy Kober, Eliza Bliss-Moreau, and Lisa Feldman Barrett. The brain basis of emotion: a meta-analytic review. The Behavioral and Brain Sciences, 35(3):121-43, June 2012. ISSN 1469-1825. doi: 10.1017/S0140525X11000446. URL http://www.ncbi.nlm.nih.gov/pubmed/22617651.

Klaus Linkenkaer-Hansen, Vadim V Nikulin, Satu Palva, Risto J Ilmoniemi, and $\mathrm{J}$ Matias Palva. Prestimulus oscillations enhance psychophysical performance in humans. The Journal of Neuroscience, 24(45):10186-90, November 2004. ISSN 15292401. doi: 10.1523/JNEUROSCI.2584-04.2004. URL http://ww.ncbi.nlm.nih.gov/ pubmed/15537890.

Feng Liu, Wenbin Guo, Dengmiao Yu, Qing Gao, Keming Gao, Zhimin Xue, Handan Du, Jianwei Zhang, Changlian Tan, Zhening Liu, Jingping Zhao, and Huafu Chen. Classification of Different Therapeutic Responses of Major Depressive Disorder with Multivariate Pattern Analysis Method Based on Structural MR Scans. PLOS ONE, 7(7):e40968, July 2012. ISSN 1932-6203. doi: 10.1371/journal.pone. 0040968. URL http://ww.pubmedcentral.nih.gov/articlerender.fcgi?artid= $3398877 \&$ tool=pmcentrez\&rendertype=abstract .

Xun Liu, Jacqueline Hairston, Madeleine Schrier, and Jin Fan. Common and distinct networks underlying reward valence and processing stages: a meta-analysis of functional neuroimaging studies. Neuroscience and Biobehavioral Reviews, 35(5):1219-36, April 2011. ISSN 1873-7528. doi: 10.1016/j.neubiorev.2010.12.012. URL http://www.ncbi. nlm.nih.gov/pubmed/21185861.

R Llorente, P Villa, EM Marco, and MP Viveros. Analyzing the effects of a single episode of neonatal maternal deprivation on metabolite profiles in rat brain: a proton NMR spectroscopy study. Neuroscience, 201:12-19, 2012. URL http://ww. sciencedirect. $\mathrm{com} / \mathrm{science/article/pii/S0306452211013005.}$

J. Logan, J.S. Fowler, N.D. Volkow, G.J. Wang, Y.S. Ding, and D.L. Alexoff. Distribution volume ratios without blood sampling from graphical analysis of PET data. Journal of 
Cerebral Blood Flow \& Metabolism, 16(5):834-840, 1996. LRL ht tp://www . nature.com/ jcbfm/journal/v16/n5/abs/9590104a.html.

Nikos K Logothetis. The neural basis of the blood-oxygen-level-dependent functional magnetic resonance imaging signal. Philosophical Transactions of the Royal Society of London. Series B, Biological Sciences, 357(1424):1003-37, August 2002. ISSN 0962-8436. doi: 10.1098/rstb.2002.1114. URL http://ww .pubmedcentral.nih.gov/articlerender. fcgi?artid=1693017\&tool=pmcentrez\&rendertype=abstract.

Anton Lord, Dorothea Horn, Michael Breakspear, and Martin Walter. Changes in community structure of resting state functional connectivity in unipolar depression. PloS One, 7(8):e41282, January 2012. ISSN 1932-6203. doi: 10.1371/journal.pone.0041282. URL http://www.ncbi.nlm.nih.gov/pubmed/22916105.

J Louvel, C Papatheodoropoulos, A Siniscalchi, I Kurcewicz, R Pumain, B Devaux, B Turak, V Esposito, J G Villemeure, and M Avoli. GABA-mediated synchronization in the human neocortex: elevations in extracellular potassium and presynaptic mechanisms. Neuroscience, 105(4):803-13, January 2001. ISSN 0306-4522. URL http://www.ncbi.nlm.nih.gov/pubmed/11530219.

Natasja J G Maandag, Daniel Coman, Basavaraju G Sanganahalli, Peter Herman, Arien J Smith, Hal Blumenfeld, Robert G Shulman, and Fahmeed Hyder. Energetics of neuronal signaling and fMRI activity. Proceedings of the National Academy of Sciences of the United States of America, 104(51):20546-51, December 2007. ISSN 1091-6490. doi: 10.1073/pnas.0709515104. URL http://www pubmedcentral.nih.gov/articlerender. fcgi ?artid=2154468\&tool=pmcentrez\&rendertype=abstract.

Jean-Philippe Mailhot, Etienne Vachon-Presseau, Philip L Jackson, and Pierre Rainville. Dispositional empathy modulates vicarious effects of dynamic pain expressions on spinal nociception, facial responses and acute pain. The European Journal of Neuroscience, 35 (2):271-8, January 2012. ISSN 1460-9568. doi: 10.1111/j.1460-9568.2011.07953.x. URL http://www.ncbi.nlm.nih.gov/pubmed/22250816.

Caterina Mainero, Jasmine Boshyan, and Nouchine Hadjikhani. Altered functional magnetic resonance imaging resting-state connectivity in periaqueductal gray networks in 
migraine. Annals of Neurology, 70(5):838-45, November 2011. ISSN 1531-8249. doi: 10.1002/ana.22537. URL http://ww .ncbi.nlm.nih.gov/pubmed/22162064.

M Majer, UM Nater, JM Lin, L Capuron, and WC Reeves. Association of childhood trauma with cognitive function in healthy adults: a pilot study. BMC Neurology, 10:61, 2010. URL http://www. doaj .org/doaj?func=abstract\&amp; id=585188.

Silvia Mangia, Ivan Tkác, Rolf Gruetter, Pierre-Francois Van de Moortele, Bruno Maraviglia, and Kâmil Ugurbil. Sustained neuronal activation raises oxidative metabolism to a new steady-state level: evidence from $1 \mathrm{H}$ NMR spectroscopy in the human visual cortex. Journal of Cerebral Blood Flow and Metabolism, 27(5):1055-63, May 2007. ISSN 0271-678X. doi: 10.1038/sj.jcbfm.9600401. URL http://www.ncbi.nlm.nih.gov/ pubmed/17033694.

Gerard J Marek. Metabotropic glutamate2/3 (mGlu2/3) receptors, schizophrenia and cognition. European Journal of Pharmacology, 639(1-3):81-90, August 2010. ISSN 18790712. doi: 10.1016/j.ejphar.2010.02.058. URL http://ww.ncbi.nlm.nih.gov/pubmed/ 20371229.

Elyssa B Margolis, Hagar Lock, Vladimir I Chefer, Toni S Shippenberg, Gregory O Hjelmstad, and Howard L Fields. Kappa opioids selectively control dopaminergic neurons projecting to the prefrontal cortex. Proceedings of the National Academy of Sciences of the United States of America, 103(8):2938-42, February 2006. ISSN 0027-8424. doi: 10.1073/pnas.0511159103. URL http://ww . pubmedcentral . nih.gov/articlerender . fcgi? artid=1413839\&tool=pmcentrez\&rendertype=abstract .

Daniel S Margulies, a M Clare Kelly, Lucina Q Uddin, Bharat B Biswal, F Xavier Castellanos, and Michael $\mathrm{P}$ Milham. Mapping the functional connectivity of anterior cingulate cortex. NeuroImage, 37(2):579-88, August 2007. ISSN 1053-8119. doi: 10.1016/j. neuroimage.2007.05.019. URL http://www.ncbi .nlm.nih.gov/pubmed/17604651.

Daniel S Margulies, Justin L Vincent, Clare Kelly, Gabriele Lohmann, Lucina Q Uddin, Bharat B Biswal, Arno Villringer, F Xavier Castellanos, Michael P Milham, and Michael Petrides. Precuneus shares intrinsic functional architecture in humans and monkeys. Proceedings of the National Academy of Sciences of the United States of 
America, 106(47):20069-74, November 2009. ISSN 1091-6490. doi: 10.1073/pnas. 0905314106. URL http://ww . pubmedcentral nih.gov/articlerender.fcgi?artid= $2775700 \&$ tool =pmcentrez\&rendertype=abstract .

M. Marjanska, P-G. Henry, E. J. Auerbach, D. Franc, B. Mueller, K. Ugurbil, and K. O. Lim. Reproducibility of In Vivo GABA Quantification in Anterior Cingulate at 3 Tesla. Proc. Intl. Soc. Mag. Reson. Med, 15, 2007.

Nicola Martini, Danilo Menicucci, Laura Sebastiani, Remo Bedini, Alessandro Pingitore, Nicola Vanello, Mattco Milanesi, Luigi Landini, and Angelo Gemignani. The dynamics of EEG gamma responses to unpleasant visual stimuli: From local activity to functional connectivity. NeuroImage, 60(2):922-32, January 2012. ISSN 10538119. doi: 10.1016/j. neuroimage.2012.01.060. URL http://www.ncbi.nlm.nih.gov/pubmed/22270349.

Esther Marx, Thomas Stephan, Annina Nolte, Angela Deutschländer, Klaus C Seelos, Marianne Dieterich, and Thomas Brandt. Eye closure in darkness animates sensory systems. NeuroImage, 19(3):924-34, July 2003. ISSN 1053-8119. doi: 10. 1016/S1053-8119(03)00150-2. URL http://linkinghub.elsevier.com/retrieve/pii/ S1053811903001502http://www.ncbi.nlm.nih.gov/pubmed/12880821.

Esther Marx, Angela Deutschländer, Thomas Stephan, Marianne Dieterich, Martin Wiesmann, and Thomas Brandt. Eyes open and eyes closed as rest conditions: impact on brain activation patterns. NeuroImage, 21(4):1818-24, April 2004. ISSN 1053-8119. doi: 10.1016/j.neuroimage.2003.12.026. URL http://www.ncbi.nlm.nih.gov/pubmed/ 15050602 .

G. Massaweh, E. Schirmacher, C. la Fougere, M. Kovacevic, C. Wangler, D. Jolly, P. Gravel, A.J. Reader, A. Thiel, and R. Schirrmacher. Improved work-up procedure for the production of $[18 \mathrm{~F}]$ flumazenil and first results of its use with a high-resolution rescarch tomograph in human stroke. Nuclear Medicine and Biology, 36(7):721-727, 2009. URL http://www.sciencedirect.com/science/article/pii/S0969805109001498.

F Matrisciano, P Tueting, S Maccari, F Nicoletti, and A Guidotti. Pharmacological Activation of Group-II Metabotropic Glutamate Receptors Corrects a SchizophreniaLike Phenotype Induced by Prenatal Stress in Mice. Neuropsychopharmacology, 37 
(4):929-38, 2011. CRL http://wwb.nature.com/npp/journal/vaop/ncurrent/full/ npp2011274a.html.

D Matsumoto and P Ekman. Japanese and Caucasian facial expressions of emotion (JACFEE). San Francisco State University, San Francisco, 1988.

Mark D Mcdonnell and Lawrence $M$ Ward. The benefits of noise in neural systems: bridging theory and experiment. Nature Reviews Neuroscience, 12(July):415425, 2011. URL http://dx.doi.org/10.1038/nrn3061http://ww .nature.com/nrn/ journal/v12/n7/abs/nrn3061.html.

Alexander McFarlane, Christopher R Clark, Richard a Bryant, Leanne M Williams, Raymond Niaura, Robert H Paul, Brian L Hitsman, Laura Stroud, David M Alexander, and Evian Gordon. The impact of early life stress on psychophysiological, personality and behavioral measures in 740 non-clinical subjects. Journal of Integrative Neuroscience, 4 (1):27-40, March 2005. ISSN 0219-6352. URL http://www.ncbi.nlm.nih.gov/pubmed/ 16035139 .

Anthony Randal McIntosh, Natasa Kovacevic, and Roxane J Itier. Increased brain signal variability accompanies lower behavioral variability in development. PLoS Computational Biology, 4(7):e1000106, January 2008. ISSN 1553-7358. doi: 10.1371/journal.pcbi. 1000106. URL http://ww. pubmedcentral.nih.gov/articlerender.fcgi?artid= $2429973 \&$ tool=pmcentrez\&rendert ype=abstract.

Maarten Mennes, Clare Kelly, Xi-Nian Zuo, Adriana Di Martino, Bharat B Biswal, $\mathrm{F}$ Xavier Castellanos, and Michael $\mathrm{P}$ Milham. Inter-individual differences in resting-state functional connectivity predict task-induced BOLD activity. NeuroImage, 50(4):1690-701, May 2010. ISSN 1095-9572. doi: 10.1016/j.neuroimage. 2010.01.002. URL http://ww . pubmedcentral.nih.gov/articlerender.fcgi?artid= $2839004 \&$ tool=pmcentrez\&rendertype=abstract.

Ravi S Menon. The great brain versus vein debate. NeuroImage, 62(2):970-74, September 2011. ISSN 1095-9572. doi: 10.1016/j.neuroimage.2011.09.005. URL http://www.ncbi. nlm.nih.gov/pubmed/21939776.

Vinod Menon and Lucina Q Uddin. Saliency, switching, attention and control: a network 
model of insula function. Brain Structure \& Function, 214(5-6):655-67, June 2010. ISSN 1863-2661. doi: 10.1007/s00429-010-0262-0. LRL http://ww pubmedcentral.nih. gov/articlerender.fcgi ?art $i d=2899886 \&$ tool=pmcent rez\&rendertype=abstract.

M. Mescher, Rolf Gruetter, H. Merkle, and M. Garwood. Water suppression using selective echo dephasing. In Proceedings of the ISMRM 4th Scientific Meeting and Exhibition, page $384,1996$.

M. Mescher, H. Merkle, J. Kirsch, M. Garwood, and R. Gruetter. Simultaneous in vivo spectral editing and water suppression. NMR in Biomedicine, 11(6):266-272, 1998. URL http://phys. columbia.edu/ tosti/icidr/cm/spec/mega_garwood.pdf.

M M Mesulam and E J Mufson. Insula of the old world monkey. I. Architectonics in the insulo-orbito-temporal component of the paralimbic brain. The Journal of Comparative Neurology, 212(1):1-22, November 1982. ISSN 0021-9967. doi: 10.1002/cne.902120102. URL http://ww.ncbi.nlm.nih.gov/pubmed/7174905.

David Meunier, Renaud Lambiotte, and Edward T Bullmore. Modular and hierarchically modular organization of brain networks. Frontiers in Neuroscience, 4:200, January 2010. ISSN 1662-453X. doi: 10.3389/fnins.2010.00200. URL http://www.pubmedcentral .nih. gov/articlerender.fcgi? artid=3000003\&tool=pmcentrez\&rendertype=abstract.

LG Miller, DJ Greenblatt, JG Barnhill, W.R. Summer, and R.I. Shader. 'GABA shift' in vivo: enhancement of benzodiazepine binding in vivo by modulation of endogenous GABA. European Journal of Pharmacology, 148:123-130, 1988. URL http: //www.sciencedirect.com/science/article/pii/001429998890461X.

Bratislav Misić, Travis Mills, Margot J Taylor, and Anthony R McIntosh. Brain noise is task dependent and region specific. Jourmal of Neurophysiology, 104(5):2667-76, November 2010. ISSN 1522-1598. doi: 10.1152/jn.00648.2010. URL http://www.ncbi.nlm.nih. gov/pubmed/20844116.

Mario Enrique Molina, Roberto Isoardi, Marcela Nathalie Prado, and Silvia Bentolila. Basal cerebral glucose distribution in long-term post-traumatic stress disorder. The World Journal of Biological Psychiatry, 11(2 Pt 2):493-501, March 2010. ISSN 1814-1412. doi: 10.3109/15622970701472094. URL http://www.ncbi.nlm.nih.gov/pubmed/20218804. 
Peter J Morgane, Janina R Galler, and David J Mokler. A review of systems and networks of the limbic forebrain/limbic midbrain. Progress in Neurobiology, 75(2):143-60, February 2005. ISSN 0301-0082. doi: 10.1016/j.pneurobio.2005.01.001. URL http://ww .ncbi. nlm.nih.gov/pubmed/15784304.

SC Mueller, FS Maheu, M Dozier, E Peloso, D Mandell, E Leibenluft, DS Pine, and $M$ Ernst. Early-life stress is associated with impairment in cognitive control in adolescence: an fMRI study. Neuropsychologia, 48(10):3037-44, 2010. URL http://www. sciencedirect.com/science/article/pii/S0028393210002423.

E J Mufson and M M Mesulam. Insula of the old world monkey. II: Afferent cortical input and comments on the claustrum. The Journal of Comparative Neurology, 212 (1):23-37, November 1982. ISSN 0021-9967. doi: 10.1002/cne.902120103. URL http: //ww.ncbi.nlm.nih.gov/pubmed/7174906.

Francesco Musso, Jürgen Brinkmeyer, Daniel Ecker, Markus K London, Giesela Thieme, Tracy Warbrick, Hans-Jörg Wittsack, Andreas Saleh, Wolfgang Greb, Peter de Boer, and Georg Winterer. Ketamine effects on brain function - Simultaneous MRI/EEG during a visual oddball task. NeuroImage, 58(2):508-25, June 2011. ISSN 1095-9572. doi: 10.1016/ j.neuroimage.2011.06.045. URL http://www.ncbi.nlm.nih.gov/pubmed/21723949.

S. D. Muthukumaraswamy, R. A.E. Edden, D. K. Jones, J. B. Swettenham, and K. D. Singh. Resting GABA concentration predicts peak gamma frequency and fMRI amplitude in response to visual stimulation in humans. Proceedings of the National Academy of Sciences of the United States of America, 106(20):8356-8361, May 2009. ISSN 0027-8424. doi: 10.1073/pnas.0900728106. URL http://www pubmedcentral.nih.gov/articlerender. fcgi ?artid=2688873\&tool=pmcentrez\&rendertype=abstract.

M Nagai, K Kishi, and S Kato. Insular cortex and neuropsychiatric disorders: a review of recent literature. European Psychiatry, 22(6):387-94, September 2007. ISSN 09249338. doi: 10.1016/j.eurpsy.2007.02.006. URL http://www.ncbi.nlm.nih.gov/pubmed/ 17416488.

Y Nagai, HD Critchley, E Featherstone, MR Trimble, and RJ Dolan. Activity in ventromedial prefrontal cortex covaries with sympathetic skin conductance level: a physiological 
account of a "default mode" of brain function. Neuroimage, 22(1):243-51, 2004. URL http://ww. sciencedirect.com/science/article/pii/S1053811904000540.

EC Nelson, AC Heath, PAF Madden, ML Cooper, SH Dinwiddie, KK Bucholz, A Glowinski, T McLaughlin, MP Dunne, DJ Statham, and NG Martin. Association between selfreported childhood sexual abuse and adverse psychosocial outcomes: results from a twin study. Archives of General Psychiatry, 59(2):139-45, 2002. URL http://archpsyc. ama-assn.org/cgi/content/abstract/59/2/139.

JB Barbosa Neto, PA Tiba, CB Faturi, EF de Castro-Neto, M da Graça Naffah-Mazacoratti, J de Jesus Mari, MF de Mello, and D Suchecki. Stress during development alters anxietylike behavior and hippocampal neurotransmission in male and female rats. Neuropharmacology, 62(1):518-526, 2011. URL http://ww. sciencedirect.com/science/article/ $\mathrm{pii/S0028390811004059.}$

M E J Newman. Modularity and community structure in networks. Proceedings of the $\mathrm{Na-}$ tional Academy of Sciences of the United States of America, 103(23):8577-82, June 2006. ISSN 0027-8424. doi: 10.1073/pnas.0601602103. URL http://www pubmedcentral .nih. gov/articlerender.fcgi ?artid=1482622\&tool=pmcentrez\&rendertype=abstract.

Georg Northoff. From Emotions to Consciousness. A Neuro-Phenomenal and NeuroRelational Approach. Frontiers in Psychology, 3(August):1-17, 2012. ISSN 1664-1078. doi: 10.3389/fpsyg.2012.00303. URL http://www.frontiersin.org/Emotion_Science/ $10.3389 /$ f psyg. $2012.00303 /$ abstract.

Georg Northoff and Pengmin Qin. How can the brain's resting state activity generate hallucinations? A 'resting state hypothesis' of auditory verbal hallucinations. Schizophrenia Research, 127(1-3):202-14, April 2011. ISSN 1573-2509. doi: 10.1016/j.schres.2010.11. 009. URL http://www.ncbi.nlm.nih.gov/pubmed/21146961.

Georg Northoff, Martin Walter, Rolf F Schulte, Johannes Beck, Ulrike Dydak, Anke Henning, Heinz Boeker, Simone Grimm, and Peter Boesiger. GABA concentrations in the human anterior cingulate cortex predict negative BOLD responses in fMRI. Nature Neuroscience, 10(12):1515-7, December 2007. ISSN 1097-6256. doi: 10.1038/nn2001. URL http://www.ncbi.nlm.nih.gov/pubmed/17982452. 
Georg Northoff, Niall W Duncan, and Dave J Hayes. The brain and its resting state activity-experimental and methodological implications. Progress in Neurobiology, 92(4): 593-600, December 2010a. ISSN 1873-5118. doi: 10.1016/j.pneurobio.2010.09.002. URL http://www.ncbi.nlm.nih.gov/pubmed/20888388.

Georg Northoff, Pengmin Qin, and Takashi Nakao. Rest-stimulus interaction in the brain: a review. Trends in Neurosciences, 33(6):277-84, June 2010b. ISSN 1878-108X. doi: 10.1016/j.tins.2010.02.006. URL http://www.ncbi.nlm.nih.gov/pubmed/20226543.

Georg Northoff, Christine Wiebking, Todd Feinberg, and Jaak Panksepp. The 'resting-state hypothesis' of major depressive disorder-A translational subcortical-cortical framework for a system disorder. Neuroscience and Biobehavioral Reviews, 35:1929-1945, December 2011. ISSN 1873-7528. doi: 10.1016/j.neubiorev.2010.12.007. URL http://www.ncbi. nlm.nih.gov/pubmed/21192971.

Kevin N Ochsner and James J Gross. The cognitive control of emotion. Trends in Cognitive Sciences, 9(5):242-9, May 2005. ISSN 1364-6613. doi: 10.1016/j.tics.2005.03.010. URL http://www.ncbi.nlm.nih.gov/pubmed/15866151.

Ikuo Odano, Christer Halldin, Per Karlsson, Andrea Varrone, Anu J Airaksinen, Raisa N Krasikova, and Lars Farde. [18F]flumazenil binding to central benzodiazepine receptor studies by PET-quantitative analysis and comparisons with [11C]flumazenil. NeuroImage, 45(3):891-902, April 2009. ISSN 1095-9572. doi: 10.1016/j.neuroimage.2008.12.005. URL http://www.ncbi.nlm.nih.gov/pubmed/19136064.

$\mathrm{R} W$ Olsen. The GABA postsynaptic membrane receptor-ionophore complex. Site of action of convulsant and anticonvulsant drugs. Molecular and Cellular Biochemistry, 39:26179, September 1981. ISSN 0300-8177. URL http://www.ncbi.nlm.nih.gov/pubmed/ 6273709 .

D Ongür and J L Price. The organization of networks within the orbital and medial prefrontal cortex of rats, monkeys and humans. Cerebral Cortex, 10(3):206-19, March 2000. ISSN 1047-3211. URL http://www.ncbi.nlm.nih.gov/pubmed/10731217.

Suzanne Oosterwijk, Kristen a Lindquist, Eric Anderson, Rebecca Dautoff, Yoshiya Moriguchi, and Lisa Feldman Barrett. States of mind: Emotions, body feelings, and 
thoughts share distributed neural networks. NeuroImage, 62(3):2110-2128, June 2012. ISSN 1095-9572. doi: 10.1016/j.neuroimage.2012.05.079. URL http://www.ncbi.nlm. nih.gov/pubmed/22677148.

Sandra C Paivio and Kenneth M Cramer. Factor structure and reliability of the Childhood Trauma Questionnaire in a Canadian undergraduate student sample. Child Abuse 8 Neglect, 28(8):889-904, August 2004. ISSN 0145-2134. doi: 10.1016/j.chiabu.2004.01.011. URL http://ww.ncbi.nlm.nih.gov/pubmed/15350772.

Nicola Palomero-Gallagher, Hartmut Mohlberg, Karl Zilles, and Brent Vogt. Cytology and receptor architecture of human anterior cingulate cortex. The Journal of Comparative Neurology, 508(6):906-26, June 2008. ISSN 1096-9861. doi: 10.1002/ cne.21684. URL http://www.pubmedcentral.nih.gov/articlerender.fcgi?artid= 2678551\&tool=pmcentrez\&rendertype=abstract.

Nicola Palomero-Gallagher, Brent A Vogt, Axel Schleicher, Helen S Mayberg, and Karl Zilles. Receptor architecture of human cingulate cortex: evaluation of the four-region neurobiological model. Human Brain Mapping, 30(8):2336-55, August 2009. ISSN 1097-0193. doi: $10.1002 / \mathrm{hbm} .20667$. URL http://www.ncbi.nlm.nih.gov/pubmed/19034899.

Jaak Panksepp. Affective Neuroscience: The Foundations of Human and Animal Emotions. Oxford University Press, Oxford, 1998.

Costas Papatheodoropoulos. NMDA receptor-dependent high-frequency network oscillations (100-300 Hz) in rat hippocampal slices. Neuroscience Letters, 414(3):197202, March 2007. ISSN 0304-3940. doi: 10.1016/j.neulet.2006.10.036. URL http: //www.ncbi.nIm.nih.gov/pubmed/17316998.

PL Pearl, KM Gibson, Z Quezado, I Dustin, J Taylor, S Trzcinski, J Schreiber, K Forester, P Reeves-Tyer, C Liew, S Shamim, P Herscovitch, R Carson, J Butman, C Jakobs, and $\mathrm{W}$ Theodore. Decreased GABA-A binding on FMZ-PET in succinic semialdehyde dehydrogenase deficiency. Neurology, 73(6):423-9, 2009. URL http://www neurology. org/content $/ 73 / 6 / 423$. short.

Keram Pfeiffer and Andrew S French. GABAergic excitation of spider mechanoreceptors increases information capacity by increasing entropy rather than decreasing jit- 
ter. The Journal of Neuroscience, 29(35):10989 94, September 2009. ISSN 1529-2401. doi: 10.1523/JNELROSCI.2744-09.2009. URL http://ww .jneurosci.org/content/ 29/35/10989. long.

M E Phelps. Positron computed tomography studies of cerebral glucose metabolism in man: theory and application in nuclear medicine. Seminars in Nuclear Medicine, 11(1):32-49, January 1981. ISSN 0001-2998. URL http://www.ncbi.nlm.nih.gov/pubmed/6972094.

M Piché, M Arsenault, and P Rainville. Dissection of perceptual, motor and autonomic components of brain activity evoked by noxious stimulation. Pain, 149(3):453-62, June 2010. ISSN 1872-6623. doi: 10.1016/j.pain.2010.01.005. URL http://www.ncbi.nlm. nih.gov/pubmed/20417032.

S J Pittman and C A McAlpine. Movements of marine fish and decapod crustaceans: process, theory and application. Advances in marine biology, 44:205-94, January 2003. ISSN 0065-2881. URL http://www.ncbi.nlm.nih.gov/pubmed/12846043.

A Ploghaus, I Tracey, J S Gati, S Clare, R S Menon, P M Matthews, and J N Rawlins. Dissociating pain from its anticipation in the human brain. Science, 284(5422):1979-81, June 1999. ISSN 0036-8075. URL http://www.ncbi.nlm.nih.gov/pubmed/10373114.

G Pluck, KH Lee, and R David. Neurobehavioural and cognitive function is linked to childhood trauma in homeless adults. British Journal of Clinical Psychology, 50(1):33-45, 2011. URL http://onlinelibrary .wiley.com/doi/10.1348/014466510X490253/full.

Olga Pollatos, Rainer Schandry, Dorothee P Auer, and Christian Kaufmann. Brain structures mediating cardiovascular arousal and interoceptive awareness. Brain Research, 1141: 178-87, April 2007. ISSN 0006-8993. URL http://ww.ncbi.nlm.nih.gov/pubmed/ 17296169 .

Alexander Prehn-Kristensen, Christian Wiesner, Til Ole Bergmann, Stephan Wolf, Olav Jansen, Hubertus Maximilian Mehdorn, Roman Ferstl, and Bettina M Pause. Induction of empathy by the smell of anxiety. PloS One, 4(6):e5987, January 2009. ISSN 19326203. doi: 10.1371/journal.pone.0005987. URL http://www.pubmedcentral.nih.gov/ articlerender.f $\mathrm{cgi}$ ? artid $=2695008$ \& $00 \mathrm{l}=$ pmcentrez\&rendertype=abstract . 
Stephanie D Preston and Frans B M de Waal. Empathy: Its ultimate and proximate bases. The Behavioral and Brain Sciences, 25(1):1-20; discussion 20-71, February 2002. ISSN 0140-525X. URL http://ww.ncbi.nlm.nih.gov/pubmed/12625087.

M C Prevett, A A Lammertsma, D J Brooks, P A Bartenstein, P N Patsalos, D R Fish, and J S Duncan. Benzodiazepine-GABAA receptors in idiopathic generalized epilepsy measured with [11C]flumazenil and positron emission tomography. Epilepsia, 36(2):11321, February 1995. ISSN 0013-9580. URL http://wwh.ncbi.nlm.nih.gov/pubmed/ 7821267.

Joseph L Price. Definition of the orbital cortex in relation to specific connections with limbic and visceral structures and other cortical regions. Annals of the New York Academy of Sciences, 1121:54-71, December 2007. ISSN 0077-8923. doi: 10.1196/annals.1401.008. URL http://www.ncbi.nlm.nih.gov/pubmed/17698999.

WG Proctor. The dependence of a nuclear magnetic resonance frequency upon chemical compound. Physical Review, 77:717, 1950. URL http://prola.aps.org/abstract/PR/ v77/i5/p717_1.

S W Provencher. Estimation of metabolite concentrations from localized in vivo proton NMR spectra. Magnetic Resonance in Medicine, 30(6):672-9, December 1993. ISSN 0740-3194. URL http://www.ncbi.nlm.nih.gov/pubmed/8139448.

S W Provencher. Automatic quantitation of localized in vivo $1 \mathrm{H}$ spectra with LCModel. NMR in Biomedicine, 14(4):260-4, June 2001. ISSN 0952-3480. URL http://www. ncbi. nlm.nih.gov/pubmed/11410943.

M E Raichle, A M MacLeod, A Z Snyder, W J Powers, D A Gusnard, and G L Shulman. A default mode of brain function. Proceedings of the National Academy of Sciences of the United States of America, 98(2):676-82, January 2001. ISSN 0027-8424. doi: 10. 1073/pnas.98.2.676. URL http://www . pubmedcentral.nih.gov/articlerender.fcgi? artid $=14647 \&$ tool $=$ pmcentrez\&rendertype=abstract .

ME Raichle. A paradigm shift in functional brain imaging. The Journal of Neuroscience, 29 (41):12729-34, October 2009. ISSN 1529-2401. doi: 10.1523/JNEUROSCI.4366-09.2009. URL http://www.ncbi.nlm.nih.gov/pubmed/19828783. 
Anjali Raja Beharelle, Natasa Kovačević, Anthony R McIntosh, and Brian Levine. Brain signal variability relates to stability of behavior after recovery from diffuse brain injury. NeuroImage, 60(2):1528-37, April 2012. ISSN 1095-9572. doi: 10.1016/j.neuroimage. 2012.01.037. URL http://www.ncbi.nlm.nih.gov/pubmed/22261371.

Geraint Rees. Neural correlates of the contents of visual awareness in humans. Philosophical Transactions of the Royal Society of London. Series B, Biological Sciences, 362(1481):877-86, May 2007. ISSN 0962-8436. doi: 10.1098/rstb. 2007.2094. URL http://www.pubmedcentral nih.gov/articlerender.fcgi?artid= $2430003 \&$ tool=pmcentrez\&rendertype=abstract.

S Reiss, RA Peterson, DM Gursky, and RJ McNally. Anxiety sensitivity, anxiety frequency and the prediction of fearfulness. Behaviour Research and Therapy, 24(1):1-8, 1986. URL http://www.sciencedirect.com/science/article/pii/0005796786901439.

Jeremy R Reynolds, Randall C O'Reilly, Jonathan D Cohen, and Todd S Braver. The function and organization of lateral prefrontal cortex: a test of competing hypotheses. PloS One, 7(2):e30284, January 2012. ISSN 1932-6203. doi: 10.1371/journal.pone.0030284. URL http://dx.plos.org/10.1371/journal . pone.0030284.

Carlos Riaza Bermudo-Soriano, M Mercedes Perez-Rodriguez, Concepcion VaqueroLorenzo, and Enrique Baca-Garcia. New perspectives in glutamate and anxiety. Pharmacology, Biochemistry, and Behavior, 100(4):752-74, February 2012. ISSN 1873-5177. doi: 10.1016/j.pbb.2011.04.010. URL http://www.ncbi.nlm.nih.gov/pubmed/21569789.

Joshua S Richman and J Randall Moorman. Physiological time-series analysis using approximate entropy and sample entropy. Am J Physiol Heart Circ Physiol, 278:H2039-H2049, 2000.

Angela C Roberts, Davorka L Tomic, Caroline H Parkinson, Tom A Roeling, David J Cutter, Trevor W Robbins, and Barry J Everitt. Forebrain connectivity of the prefrontal cortex in the marmoset monkey (Callithrix jacchus): an anterograde and retrograde tract-tracing study. The Journal of Comparative Neurology, 502(1):86-112, May 2007. ISSN 0021-9967. doi: 10.1002/cne.21300. URL http://www.ncbi.nlm.nih.gov/pubmed/ 17335041. 
Osvaldo A Rosso. Entropy changes in brain function. International Journal of Psychophysiology, 64(1):75-80, April 2007. ISSN 0167-8760. doi: 10.1016/j.ijpsycho.2006.07.010. URL http://www.ncbi.nlm.nih.gov/pubmed/17234291.

Guillaume a. Rousselet and Cyril R. Pernet. Improving standards in brain-behavior correlation analyses. Frontiers in Human Neuroscience, 6(May), 2012. ISSN 1662-5161. doi: 10.3389/fnhum.2012.00119. URL http://www.frontiersin.org/Human_Neuroscience/ $10.3389 /$ f nhum. $2012.00119 /$ abstract.

Mathieu Roy, Daphna Shohamy, and Tor D Wager. Ventromedial prefrontal-subcortical systems and the generation of affective meaning. Trends in Cognitive Sciences, 16(3): 147-56, March 2012. ISSN 1879-307X. doi: 10.1016/j.tics.2012.01.005. URL http: //www.ncbi.nlm.nih.gov/pubmed/22310704.

Mikail Rubinov and Olaf Sporns. Complex network measures of brain connectivity: uses and interpretations. Neurolmage, 52(3):1059-69, September 2010. ISSN 1095-9572. doi: 10.1016/j.neuroimage.2009.10.003. URL http://ww.ncbi.nlm.nih.gov/pubmed/ 19819337.

Perrine Ruby and Jean Decety. How would you feel versus how do you think she would feel? A neuroimaging study of perspective-taking with social emotions. Journal of Cognitive Neuroscience, 16(6):988-99, 2004. ISSN 0898-929X. doi: 10.1162/0898929041502661. URL http://www.ncbi.nlm.nih.gov/pubmed/15298786.

J Rudolf, J Sobesky, M Ghaemi, and W-D Heiss. The correlation between cerebral glucose metabolism and benzodiazepine receptor density in the acute vegetative state. European Journal of Neurology, 9(6):671-7, November 2002. ISSN 1351-5101. URL http://www. ncbi.nlm.nih.gov/pubmed/12453084.

B Ryan, L Musazzi, A Mallei, D Tardito, SHM Gruber, A El Khoury, R Anwyl, G Racagni, AA Mathe, MJ Rowan, and M Popoli. Remodelling by early-life stress of NMDA receptordependent synaptic plasticity in a gene-environment rat model of depression. The International Journal of Neuropsychopharmacology, 12:553-559, 2009. URL http://journals. cambridge.org/production/action/cjoGetFull text? fulltextid=5475072.

NN Ryzhikov, N Seneca, and RN Krasikova. Preparation of highly specific radioactivity 
flumazenil and its evaluation in cynomolgus monkey by positron emission tomography. Nuclear Medicine and Biology, 32(2):109-16, 2005. CRL http://www.sciencedirect. com/science/article/pii/S0969805104001672.

Sepideh Sadaghiani, Guido Hesselmann, Karl J Friston, and Andreas Kleinschmidt. The relation of ongoing brain activity, evoked neural responses, and cognition. Frontiers in Systems Neuroscience, 4(June):20, January 2010. ISSN 1662-5137. doi: 10.3389/fnsys. 2010.00020. URL http://www.pubmedcentral.nih.gov/articlerender.fcgi?artid= $2903187 \&$ tool=pmcentrez\&rendert ype=abstract .

Elina Salmi, Sargo Aalto, Jussi Hirvonen, Jaakko W Lå ngsjö, Anu T Maksimow, Vesa Oikonen, Liisa Metsähonkala, Jussi Virkkala, Kjell Nả gren, and Harry Scheinin. Measurement of GABAA receptor binding in vivo with [11C]flumazenil: a test-retest study in healthy subjects. NeuroImage, 41(2):260-9, June 2008. ISSN 1053-8119. doi: 10.1016/ j.neuroimage.2008.02.035. URL http://www.ncbi.nlm.nih.gov/pubmed/18411060.

Giacomo Salvadore, Brian R Cornwell, Veronica Colon-Rosario, Richard Coppola, Christian Grillon, Carlos A Zarate, and Husseini $\mathrm{K}$ Manji. Increased anterior cingulate cortical activity in response to fearful faces: a neurophysiological biomarker that predicts rapid antidepressant response to ketamine. Biological Psychiatry, 65(4):289-95, February 2009. ISSN 1873-2402. doi: 10.1016/j.biopsych. 2008.08.014. URL http://ww. pubmedcentral.nih.gov/articlerender.fcgi?artid= $2643469 \&$ tool=pmcentrez\&rendertype=abstract.

Giacomo Salvadore, Brian R Cornwell, Fabio Sambataro, David Latov, Veronica ColonRosario, Frederick Carver, Tom Holroyd, Nancy DiazGranados, Rodrigo Machado-Vieira, Christian Grillon, Wayne C Drevets, and Carlos A Zarate. Anterior cingulate desynchronization and functional connectivity with the amygdala during a working memory task predict rapid antidepressant response to ketamine. Neuropsychopharmacology, 35(7):1415-22, June 2010. ISSN 1740-634X. doi: 10.1038/npp.2010.24. URL http://dx.doi.org/10.1038/npp.2010.24.

Michael Salzberg, Gaurav Kumar, Laureen Supit, Nigel C Jones, Margaret J Morris, Sandra Rees, and Terence J O'Brien. Early postnatal stress confers enduring vulnerability to limbic epileptogenesis. Epilepsia, 48(11):2079-85, November 2007. ISSN 0013-9580. 
doi: 10.1111/j.1528-1167.2007.01246.x. URL http://www.ncbi.nlm.nih.gov/pubmed/ 17999745 .

I Savic, E Svanborg, and J O Thorell. Cortical benzodiazepine receptor changes are related to frequency of partial seizures: a positron emission tomography study. Epilepsia, 37(3): 236-44, March 1996. ISSN 0013-9580. URL http://www.ncbi.nlm.nih.gov/pubmed/ 8598181 .

Zeynep M Saygin, David E Osher, Kami Koldewyn, Gretchen Reynolds, John D E Gabrieli, and Rebecca R Saxe. Anatomical connectivity patterns predict face selectivity in the fusiform gyrus. Nature Neuroscience, 15(2):321-327, December 2012. ISSN 1546-1726. doi: 10.1038/nn.3001. URL http://www.ncbi.nlm.nih.gov/pubmed/22197830.

Heath D Schmidt and R Christopher Pierce. Cocaine-induced neuroadaptations in glutamate transmission: potential therapeutic targets for craving and addiction. Annals of the New York Academy of Sciences, 1187:35-75, February 2010. ISSN 1749-6632. doi: 10.1111/j.1749-6632.2009.05144.x. URL http://www.ncbi.nlm.nih.gov/pubmed/ 20201846 .

William W Seeley, Vinod Menon, Alan F Schatzberg, Jennifer Keller, Gary H Glover, Heather Kenna, Allan L Reiss, and Michael D Greicius. Dissociable intrinsic connectivity networks for salience processing and executive control. The Journal of Neuroscience, 27(9):2349-56, February 2007. ISSN 1529-2401. doi: 10.1523/JNEUROSCI.5587-06.2007. URL http://ww . pubmedcentral.nih.gov/ articlerender.fcgi ?artid=2680293\&tool=pmcentrez\&rendertype=abstract.

YS Sheu, A Polcari, CM Anderson, and MH Teicher. Harsh corporal punishment is associated with increased $\mathrm{T} 2$ relaxation time in dopamine-rich regions. Neurolmage, 53(2):412-419, 2010. URL http://www.sciencedirect.com/science/article/pii/ S1053811910008876.

Lisa M Shin and Israel Liberzon. The neurocircuitry of fear, stress, and anxiety disorders. Neuropsychopharmacology, 35(1):169-91, January 2010. ISSN 1740-634X. doi: 10.1038/ npp.2009.83. URL http://www.pubmedcentral .nih.gov/articlerender.fcgi?artid= 3055419\&tool=pmcentrez\&rendertype=abstract. 
Lisa M Shin, Christopher I Wright, Paul A Cannistraro, Michelle M Wedig, Katherine McMullin, Brian Martis, Michael L Macklin, Natasha B Lasko, Sarah R Cavanagh, Terri S Krangel, Scott P Orr, Roger K Pitman, Paul J Whalen, and Scott L Rauch. A functional magnetic resonance imaging study of amygdala and medial prefrontal cortex responses to overtly presented fearful faces in posttraumatic stress disorder. Archives of General Psychiatry, 62(3):273-81, March 2005. ISSN 0003-990X. doi: 10.1001/archpsyc.62.3.273. URL http://ww. ncbi.nlm.nih.gov/pubmed/15753240.

LM Shin and $\mathrm{K}$ Handwerger. Is posttraumatic stress disorder a stress induced fear circuitry disorder? Journal of Traumatic Stress, 22(5):409-15, 2009. URL http: //onlinelibrary.wiley.com/doi/10.1002/jts.20442/abstract.

LM Shin, SP Orr, MA Carson, SL Rauch, ML Macklin, NB Lasko, PM Peters, LJ Metzger, DD Dougherty, PA Cannistraro, NM Alpert, AJ Fischman, and RK Pitman. Regional cerebral blood flow in the amygdala and medial prefrontal cortex during traumatic imagery in male and female Vietnam veterans with PTSD. Archives of General Psychiatry, 61(2):168-76, 2004. URL http://archpsyc.ama-assn.org/cgi/content/ abstract/61/2/168.

Greg J Siegle, Wesley Thompson, Cameron S Carter, Stuart R Steinhauer, and Michael E Thase. Increased amygdala and decreased dorsolateral prefrontal BOLD responses in unipolar depression: related and independent features. Biological Psychiatry, 61(2):198209, January 2007. ISSN 0006-3223. doi: 10.1016/j.biopsych.2006.05.048. URL http: //www.ncbi.nlm.nih.gov/pubmed/17027931.

E. Sigel. Mapping of the benzodiazepine recognition site on GABA-A receptors. Current Topics in Medicinal Chemistry, 2(8):833-839, 2002. URL http://www ingentaconnect. $\mathrm{com} /$ content/ben/ctmc/2002/00000002/00000008/art00004.

W Kyle Simmons, Jason a Avery, Joel C Barcalow, Jerzy Bodurka, Wayne C Drevets, and Patrick Bellgowan. Keeping the body in mind: Insula functional organization and functional connectivity integrate interoceptive, exteroceptive, and emotional awareness. Human Brain Mapping, June 2012. ISSN 1097-0193. doi: 10.1002/hbm.22113. URL http://www.ncbi.nlm.nih.gov/pubmed/22696421. 
J R Simpson, W C Drevets, A Z Snyder, D A Gusnard, and M E Raichle. Emotioninduced changes in human medial prefrontal cortex: II. During anticipatory anxiety. Proceedings of the National Academy of Sciences of the United States of America, 98(2):688-93, January 2001. ISSN 0027-8424. doi: 10.1073/pnas.98.2.688. URL http://ww . pubmedcentral.nih.gov/articlerender.fcgi?artid=14649\&tool= pmcentrez\&rendertype=abstract .

Tania Singer and Claus Lamm. The social neuroscience of empathy. Annals of the New York Academy of Sciences, 1156:81-96, March 2009. ISSN 1749-6632. doi: 10.1111/j. 1749-6632.2009.04418.x. URL http://www.ncbi.nlm.nih.gov/pubmed/19338504.

Tania Singer, Hugo D Critchley, and Kerstin Preuschoff. A common role of insula in feelings, empathy and uncertainty. Trends in Cognitive Sciences, 13(8):334-40, August 2009. ISSN 1364-6613. doi: 10.1016/j.tics.2009.05.001. URL http://www.ncbi.nlm. nih.gov/pubmed/19643659.

Pawel Skudlarski, Kanchana Jagannathan, Karen Anderson, Michael C Stevens, Vince D Calhoun, Beata A Skudlarska, and Godfrey Pearlson. Brain connectivity is not only lower but different in schizophrenia: a combined anatomical and functional approach. Biological Psychiatry, 68(1):61-9, July 2010. ISSN 1873-2402. doi: 10.1016/j.biopsych. 2010.03.035. URL http://www.pubmedcentral.nih.gov/articlerender.fcgi?artid= 2900394\& tool=pmcentrez\&rendertype=abstract.

S.M. Smith, M. Jenkinson, M.W. Woolrich, C.F. Beckmann, T.E.J. Behrens, H. JohansenBerg, P.R. Bannister, M. De Luca, I. Drobnjak, D.E. Flitney, and Others. Advances in functional and structural MR image analysis and implementation as FSL. Neuroimage, 23:S208-S219, 2004. URL http://www.sciencedirect.com/science/article/ $\mathrm{pii} / \mathrm{S} 1053811904003933$.

S.M. Smith, P.T. Fox, K.L. Miller, D.C. Glahn, P.M. Fox, C.E. Mackay, Nicola Filippini, K.E. Watkins, Roberto Toro, A.R. Laird, and Others. Correspondence of the brain's functional architecture during activation and rest. Proceedings of the National Academy of Sciences of the United States of America, 106(31):13040-13045, 2009. URL http: //www.pnas.org/content/106/31/13040. short. 
Stephen M Smith, Karla L Miller, Gholamreza Salimi-Khorshidi, Matthew Webster, Christian F Beckmann, Thomas E Nichols, Joseph D Ramsey, and Mark W Woolrich. Network modelling methods for FMRI. NeuroImage, 54(2):875-91, January 2011. ISSN 1095-9572. doi: 10.1016/j.neuroimage.2010.08.063. CRL http://ww.ncbi.nlm.nih.gov/pubmed/ 20817103.

Ilaria Spoletini, Andrea Cherubini, Giulia Banfi, Ivo Alex Rubino, Patrice Peran, Carlo Caltagirone, and Gianfranco Spalletta. Hippocampi, thalami, and accumbens microstructural damage in schizophrenia: a volumetry, diffusivity, and neuropsychological study. Schizophrenia Bulletin, 37(1):118-30, January 2011. ISSN 1745-1701. doi: 10.1093/schbul/sbp058. URL http://www.pubmedcentral.nih.gov/articlerender. fcgi?artid=3004185\&tool=pmcentrez\&rendertype=abstract.

Devarajan Sridharan, Daniel J Levitin, and Vinod Menon. A critical role for the right fronto-insular cortex in switching between central-executive and default-mode networks. Proceedings of the National Academy of Sciences of the United States of America, 105(34):12569-74, August 2008. ISSN 1091-6490. doi: 10.1073/pnas. 0800005105. URL http://ww . pubmedcentral nih.gov/articlerender.fcgi?artid= 2527952\&tool=pmcentrez\&rendertype=abstract.

Philipp Sterzer and Andreas Kleinschmidt. Anterior insula activations in perceptual paradigms: often observed but barely understood. Brain Structure \& Function, 214 (5-6):611-22, June 2010. ISSN 1863-2661. doi: 10.1007/s00429-010-0252-2. URL http://www.ncbi.nlm.nih.gov/pubmed/20512379.

J M Stone, C Dietrich, R Edden, M A Mehta, S De Simoni, L J Reed, J H Krystal, D Nutt, and G J Barker. Ketamine effects on brain GABA and glutamate levels with 1H-MRS: relationship to ketamine-induced psychopathology. Molecular Psychiatry, 17(7):664-5, January 2012. ISSN 1476-5578. doi: 10.1038/mp.2011.171. URL http://www.ncbi. nlm.nih.gov/pubmed/22212598.

K Supekar, LQ Uddin, K Prater, H Amin, MD Greicius, and V Menon. Development of functional and structural connectivity within the default mode network in young children. Neuroimage, 52(1):290-301, 2010. URL http://www.sciencedirect.com/ science/article/pii/s1053-8119(10)00403-9. 
C M Sylvester, M Corbetta, M E Raichle, T L Rodebaugh, B L Schlaggar, Y I Sheline, C F Zorumski, and E J Lenze. Functional network dysfunction in anxiety and anxiety disorders. Trends in Neurosciences, pages 1-9, June 2012. ISSN 1878-108X. doi: 10. 1016/j.tins.2012.04.012. URL http://ww.ncbi.nlm.nih.gov/pubmed/22658924.

Keri S Taylor, David A Seminowicz, and Karen D Davis. Two systems of resting state connectivity between the insula and cingulate cortex. Human Brain Mapping, 30(9): 2731-45, September 2009. ISSN 1097-0193. doi: 10.1002/hbm.20705. URL http://www . ncbi.nlm.nih.gov/pubmed/19072897.

MH Teicher, CM Anderson, and A Polcari. Childhood maltreatment is associated with reduced volume in the hippocampal subfields CA3, dentate gyrus, and subiculum. Proceedings of the National Academy of Sciences of the United States of America, 109(9): E563-72, 2012. URL http://www.pnas.org/content/109/9/E563. short.

Sibel Tekin and Jeffrey L Cummings. Frontal-subcortical neuronal circuits and clinical neuropsychiatry: an update. Journal of Psychosomatic Research, 53(2):647-54, August 2002. ISSN 0022-3999. URL http://www.ncbi.nlm.nih.gov/pubmed/12169339.

A. Thiel, A. Reilhac, D.L. Collins, O. Rousset, P. Neelin, and A.C. Evans. Volumeof-interest based automated partial volume correction for large numbers of PET. In M. Senda, Y. Kimura, and P. Herscovitch, editors, Brain Imaging Using PET, pages 129-137. Academic Press, Amsterdam, 2002. URL http://books. google. $\mathrm{ca} /$ books?hl=en\&lr=\&id=0oaHwVyRizEC\&oi=fnd\&pg=PA129\&dq=Thiel+ Interest-Based+Automated+Partial+Volume+Correction+for+Large+Numbers+ of +PET\&ots=0yNrtssi - g\&sig=HMYBC2Ioivgj $-z D I K 95 \mathrm{Acm} 77 \mathrm{TWC}$ v=onepage\&q= ThielInterest-BasedAut omatedPartialVolumeCorrectionforLargeNumbersofPET\&f= false.

K Thomaes, E Dorrepaal, N Draijer, MB de Ruiter, BM Elzinga, AJ van Balkom, JH Smit, and DJ Veltman. Treatment effects on insular and anterior cingulate cortex activation during classic and emotional Stroop interference in child abuserelated complex post-traumatic stress disorder. Psychological Medicine, pages 1-13, 2012. URL http://journals.cambridge.org/production/action/cjoGetFulltext? full textid=8518959. 
I Tkác, Z Starcuk, I Y Choi, and $\mathrm{R}$ Gruetter. In vivo $1 \mathrm{H}$ NMR spectroscopy of rat brain at $1 \mathrm{~ms}$ echo time. Magnetic Resonance in Medicine, 41(4):649-56, April 1999. ISSN 0740-3194. URL http://www.ncbi.nlm.nih.gov/pubmed/10332839.

D Tomasi and N D Volkow. Aging and functional brain networks. Molecular Psychiatry, 17(5):549-58, July 2011. ISSN 1476-5578. doi: 10.1038/mp.2011.81. URL http://www . ncbi.nlm.nih.gov/pubmed/21727896.

A Tomoda, CP Navalta, A Polcari, and N Sadato. Childhood sexual abuse is associated with reduced gray matter volume in visual cortex of young women. Biological Psychiatry, 66(7):642-8, 2009. URL http://www.sciencedirect.com/science/article/pii/ S0006322309005071.

G Turecki, C Ernst, F Jollant, B Labonte, and N Mechawar. The neurodevelopmental origins of suicidal behavior. Trends in Neurosciences, 35(1):14-23, 2012. URL http: //www.sciencedirect.com/science/article/pii/s0166223611001950.

LQ Uddin, KS Supekar, S Ryali, and V Menon. Dynamic reconfiguration of structural and functional connectivity across core neurocognitive brain networks with development. The Journal of Neurascience, 31(50):18578-89, 2011. URL http://www .jneurosci.org/ content/31/50/18578. short.

Lucina Q Uddin, Kaustubh Supekar, and Vinod Menon. Typical and atypical development of functional human brain networks: insights from resting-state FMRI. Frontiers in Systems Neuroscience, 4:21, January 2010. ISSN 1662-5137. doi: 10.3389/fnsys. 2010.00021. URL http://ww. pubmedcentral.nih.gov/articlerender.fcgi?artid= 2889680\&tool=pmcentrez\&rendertype=abstract.

Peter J Uhlhaas and Wolf Singer. The development of neural synchrony and large-scale cortical networks during adolescence: relevance for the pathophysiology of schizophrenia and neurodevelopmental hypothesis. Schizophrenia Bulletin, 37(3):514-23, May 2011. ISSN 1745-1701. doi: 10.1093/schbul/sbr034. URL http://ww .pubmedcentral.nih. gov/articlerender.fcgi?artid=3080681\&tool=pmcentrez\&rendertype=abstract.

Shankar Vallabhajosula. (18)F-labeled positron emission tomographic radiopharmaceuticals in oncology: an overview of radiochemistry and mechanisms of tumor localization. Semi- 
nars in Nuclear Medicine, 37(6):400-19, November 2007. ISSN 0001-2998. doi: 10.1053/ j.semnuclmed.2007.08.004. URL http://www.ncbi.nlm.nih.gov/pubmed/17920348.

Martijn $\mathrm{P}$ van den Heuvel and Olaf Sporns. Rich-club organization of the human connectome. The Journal of Neuroscience, 31(44):15775-86, November 2011. ISSN 15292401. doi: 10.1523/JNEUROSCI.3539-11.2011. URL http://www.ncbi.nlm.nih.gov/ pubmed/22049421.

K. R. Van Dijk, M. R. Sabuncu, and R. L. Buckner. The Influence of Head Motion on Intrinsic Functional Connectivity MRI. NeuroImage, 59(1):431-438, July 2011. ISSN 10538119. doi: 10.1016/j.neuroimage.2011.07.044. URL http://linkinghub.elsevier. com/retrieve/pii/S1053811911008214.

MJ van Tol, LR Demenescu, NJA van der Wee, R Kortekaas, MAN Marjan, JA Boer, RJ Renken, MA van Buchem, FG Zitman, A Aleman, and Veltman DJ. Functional Magnetic Resonance Imaging Correlates of Emotional Word Encoding and Recognition in Depression and Anxiety Disorders. Biological Psychiatry, 71(7):593-602, 2012. URL http://www.sciencedirect.com/science/article/pii/S0006322311011577.

Lav R Varshney, Beth L Chen, Eric Paniagua, David H Hall, and Dmitri B Chklovskii. Structural properties of the Caenorhabditis elegans neuronal network. PLoS Computational Biology, 7(2):e1001066, January 2011. ISSN 1553-7358. doi: 10.1371/journal.pcbi. 1001066. URL http://dx.plos.org/10.1371/journal.pcbi.1001066.

Teresa A Victor, Maura L Furey, Stephen J Fromm, Arne Ohman, and Wayne C Drevets. Relationship between amygdala responses to masked faces and mood state and treatment in major depressive disorder. Archives of General Psychiatry, 67 (11):1128-38, November 2010. ISSN 1538-3636. doi: 10.1001/archgenpsychiatry. 2010.144. URL http://www.pubmedcentral.nih.gov/articlerender.fcgi?artid= 3253452\&tool=pmcentrez\&rendertype=abstract.

Mansi Vithlani, Miho Terunuma, and Stephen J Moss. The dynamic modulation of GABA(A) receptor trafficking and its role in regulating the plasticity of inhibitory synapses. Physiological Reviews, 91(3):1009-22, July 2011. ISSN 1522-1210. doi: 10.1152/physrev.00015.2010. URL http://www.ncbi.nlm.nih.gov/pubmed/21742794. 
B A Vogt, E A Nimchinsky, L J Vogt, and P R Hof. Human cingulate cortex: surface features, flat maps, and cytoarchitecture. The Journal of Comparative Neurology, 359 (3):490-506, August 1995. ISSN 0021-9967. doi: 10.1002/cne.903590310. URL http: //www.ncbi.nlm.nih.gov/pubmed/7499543.

David W Volk, Stephen M Eggan, and David A Lewis. Alterations in metabotropic glutamate receptor $1 \alpha$ and regulator of $G$ protein signaling 4 in the prefrontal cortex in schizophrenia. The American Journal of Psychiatry, 167 (12):1489-98, December 2010. ISSN 1535-7228. doi: 10.1176/appi.ajp.2010. 10030318. URL http://ww .pubmedcentral.nih.gov/articlerender.fcgi?artid= 2997877\&tool=pmcentrez\&rendertype=abstract.

V Van Waes, M Enache, and A Zuena. Ethanol attenuates spatial memory deficits and increases mGlula receptor expression in the hippocampus of rats exposed to prenatal stress. Alcoholism, Clinical and Experimental Research, 33(8):1346-54, 2009. URL http: //onlinelibrary.wiley.com/doi/10.1111/j.1530-0277.2009.00964.x/full.

Martin Walter, Anke Henning, Simone Grimm, Rolf F Schulte, Johannes Beck, Ulrike Dydak, Betina Schnepf, Heinz Boeker, Peter Boesiger, and Georg Northoff. The relationship between aberrant neuronal activation in the pregenual anterior cingulate, altered glutamatergic metabolism, and anhedonia in major depression. Archives of General Psychiatry, 66(5):478-86, May 2009. ISSN 1538-3636. doi: 10.1001/archgenpsychiatry.2009.39. URL http://www.ncbi.nlm.nih.gov/pubmed/19414707.

R. Wang, T. Benner, A.G. Sorensen, and V. J. Wedeen. Diffusion Toolkit : A Software Package for Diffusion Imaging Data Processing and Tractography. In Proc. Intl. Soc. Mag. Reson. Med., volume 15, page 3720, 2007.

Marta Weinstock. Gender differences in the effects of prenatal stress on brain development and behaviour. Neurochemical Research, 32(10):1730-40, October 2007. ISSN 03643190. doi: 10.1007/s11064-007-9339-4. URL http://www.ncbi.nlm.nih.gov/pubmed/ 17406975 .

M A Whittington, R D Traub, and J G Jefferys. Synchronized oscillations in interneuron networks driven by metabotropic glutamate receptor activation. Nature, 373(6515):612- 
5, February 1995. ISSN 0028-0836. doi: 10.1038/373612a0. URL http://www.ncbi. nlm.nih.gov/pubmed/7854418.

Christine Wiebking, André Bauer, Moritz de Greck, Niall W Duncan, Claus Tempelmann, and Georg Northoff. Abnormal body perception and neural activity in the insula in depression: an fMRI study of the depressed "material me". The World Journal of Biological Psychiatry, 11(3):538-49, April 2010. ISSN 1814-1412. doi: 10.3109/15622970903563794. URL http://www.ncbi.nlm.nih.gov/pubmed/20146653.

Christine Wiebking, Moritz de Greck, Niall W Duncan, Alexander Heinzel, Claus Tempelmann, and Georg Northoff. Are emotions associated with activity during rest or interoception? An exploratory fMRI study in healthy subjects. Neuroscience Letters, 491(1):87-92, March 2011. ISSN 1872-7972. doi: 10.1016/j.neulet.2011.01.012. URL http://www.ncbi.nlm.nih.gov/pubmed/21232578.

Christine Wiebking, Niall W Duncan, Pengmin Qin, Dave J Hayes, Oliver Lyttelton, Paul Gravel, Jeroen Verhaeghe, Alexey P Kostikov, Ralf Schirrmacher, Andrew J Reader, Malek Bajbouj, and Georg Northoff. External awareness and GABA - A multimodal imaging study combining fMRI and [18F]flumazenil-PET. Human Brain Mapping, 2012.

M Wiesmann, R Kopietz, J Albrecht, J Linn, U Reime, E Kara, O Pollatos, V Sakar, A Anzinger, G Fesl, H Brückmann, G Kobal, and T Stephan. Eye closure in darkness animates olfactory and gustatory cortical areas. NeuroImage, 32(1):293-300, August 2006. ISSN 1053-8119. doi: 10.1016/j.neuroimage.2006.03.022. URL http://ww .ncbi. nlm.nih.gov/pubmed/16631383.

J C Willer. Comparative study of perceived pain and nociceptive flexion reflex in man. Pain, 3(1):69-80, February 1977. ISSN 0304-3959. URL http://www.ncbi.nlm.nih. gov/pubmed/876668.

Jonathan Winawer, Nathan Witthoft, Michael C Frank, Lisa Wu, Alex R Wade, and Lera Boroditsky. Russian blues reveal effects of language on color discrimination. Proceedings of the National Academy of Sciences of the United States of America, 104(19):7780-5, May 2007. ISSN 0027-8424. doi: 10.1073/pnas. 0701644104. URL http://ww. pubmedcentral.nih.gov/articlerender.fcgi?artid= 
$1876524 \& t o o l=$ pmcentrez\&rendertype=abstract

Georg Winterer, Michael F Egan, Bhaskar S Kolachana, Terry E Goldberg, Richard Coppola, and Daniel R Weinberger. Prefrontal electrophysiologic "noise" and catecholO-methyltransferase genotype in schizophrenia. Biological Psychiatry, 60(6):578-84, September 2006. ISSN 0006-3223. doi: 10.1016/j.biopsych.2006.03.023. URL http: //ww.ncbi.nlm.nih.gov/pubmed/16730334.

MW Woolrich, S Jbabdi, B Patenaude, and M Chappell. Bayesian analysis of neuroimaging data in FSL. NeuroImage, 45:S173-186, 2009. URL http://www.sciencedirect.com/ science/article/pii/s1053-8119(08)01204-4.

Chaogan Yan, Dongqiang Liu, Yong He, Qihong Zou, Chaozhe Zhu, Xinian Zuo, Xiangyu Long, and Yufeng Zang. Spontaneous brain activity in the default mode network is sensitive to different resting-state conditions with limited cognitive load. PloS One, 4(5):e5743, January 2009. ISSN 1932-6203. doi: 10.1371/journal.pone. 0005743. URL http://ww. pubmedcentral.nih.gov/articlerender.fcgi?artid= 2683943\&tool=pmcentrez\&rendertype=abstract.

Hong Yang, Xiang-Yu Long, Yihong Yang, Hao Yan, Chao-Zhe Zhu, Xiang-Ping Zhou, Yu-Feng Zang, and Qi-Yong Gong. Amplitude of low frequency fluctuation within visual areas revealed by resting-state functional MRI. NeuroImage, 36(1):144-52, May 2007. ISSN 1053-8119. doi: 10.1016/j.neuroimage.2007.01.054. URL http://www.ncbi.nlm. nih.gov/pubmed/17434757.

Dongyang Zhang, Abraham Z Snyder, Michael D Fox, Mark W Sansbury, Joshua S Shimony, and Marcus $E$ Raichle. Intrinsic functional relations between human cerebral cortex and thalamus. Journal of Neurophysiology, 100(4):1740-8, October 2008. ISSN 0022-3077. doi: 10.1152/jn.90463.2008. URL http://www pubmedcentral.nih.gov/ articlerender.fcgi ?artid $=2576214 \& t o 0 l=$ pmcentrez\&rendertype=abstract.

S Zhang, S Hu, HH Chao, X Luo, OM Farr, and CS Li. Cerebral correlates of skin conductance responses in a cognitive task. NeuroImage, 2012. URL http://ww. sciencedirect.com/science/article/pii/S1053811912005241.

Sheng Zhang and Chiang-shan R. Li. Functional connectivity mapping of 
the human precuneus by resting state fMRI. NeuroImage, 59(4):3548-62, November 2012. ISSN 10538119. doi: 10.1016/j.neuroimage.2011.11.023. URL http://1inkinghub.elsevier.com/retrieve/pii/S1053811911013048http:// www.ncbi.nlm.nih.gov/pubmed/22116037.

Y Zhang, M Brady, and S Smith. Segmentation of brain MR images through a hidden Markov random field model and the expectation-maximization algorithm. IEEE Transactions on Medical Imaging, 20(1):45-57, January 2001. ISSN 0278-0062. URL http://www.ncbi.nlm.nih.gov/pubmed/11293691.

Zhiqiang Zhang, Wei Liao, Huafu Chen, Dante Mantini, Ju-Rong Ding, Qiang Xu, Zhengge Wang, Cuiping Yuan, Guanghui Chen, Qing Jiao, and Guangming Lu. Altered functional-structural coupling of large-scale brain networks in idiopathic generalized epilepsy. Brain, 134(Pt 10):2912-28, October 2011. ISSN 1460-2156. doi: 10.1093/brain/awr223. URL http://brain.oxfordjournals.org.proxy. bib. uottawa. $\mathrm{ca} /$ content $/ 134 / 10 / 2912$. full? maxtoshow=\&hits=10\&RESULTFORMAT= \&fulltext=Altered+functional-structural+coupling+of+large-scale+brain + networks+in+idiopathic+generalized+epilepsy\&searchid=1\&FIRSTINDEX= O\&resourcetype $=$ HWCIT .

J Zhao, A-M Bao, X-R Qi, W Kamphuis, S Luchetti, J-S Lou, and D F Swaab. Gene expression of GABA and glutamate pathway markers in the prefrontal cortex of non-suicidal elderly depressed patients. Journal of Affective Disorders, 138(3):494-502, February 2012. ISSN 1573-2517. doi: 10.1016/j.jad.2012.01.013. URL http://www.ncbi.nlm.nih.gov/ pubmed/22357337.

Xiao-Hu Zhao, Pei-Jun Wang, Chun-Bo Li, Zheng-Hui Hu, Qian Xi, Wen-Yuan Wu, and Xiao-Wei Tang. Altered default mode network activity in patient with anxiety disorders: an fMRI study. European Journal of Radiology, 63(3):373-8, September 2007. ISSN 0720048X. doi: 10.1016/j.ejrad.2007.02.006. URL http://www.ncbi.nlm.nih.gov/pubmed/ 17400412 .

Qi-Hong Zou, Chao-Zhe Zhu, Yihong Yang, Xi-Nian Zuo, Xiang-Yu Long, Qing-Jiu Cao, Yu-Feng Wang, and Yu-Feng Zang. An improved approach to detection of amplitude of low-frequency fluctuation (ALFF) for resting-state fMRI: fractional ALFF. Journal 
of Neuroscience Methods, 172(1):137-41, July 2008. ISSN 0165-0270. doi: 10.1016/j. jneumeth.2008.04.012. URL http://www.ncbi.nlm.nih.gov/pubmed/18501969.

AR Zuena, J Mairesse, P Casolini, C Cinque, GS Alema, S Morley-Fletcher, V Chiodi, LG Spagnoli, R Gradini, A Catalini, F Nicoletti, and S Maccari. Prenatal restraint stress generates two distinct behavioral and neurochemical profiles in male and female rats. PLoS One, 3(5):e2170, 2008. URL http://dx.plos.org/10.1371/journal pone. 0002170 . 


\section{Appendix A}

Supplementary material for Chapter 2 


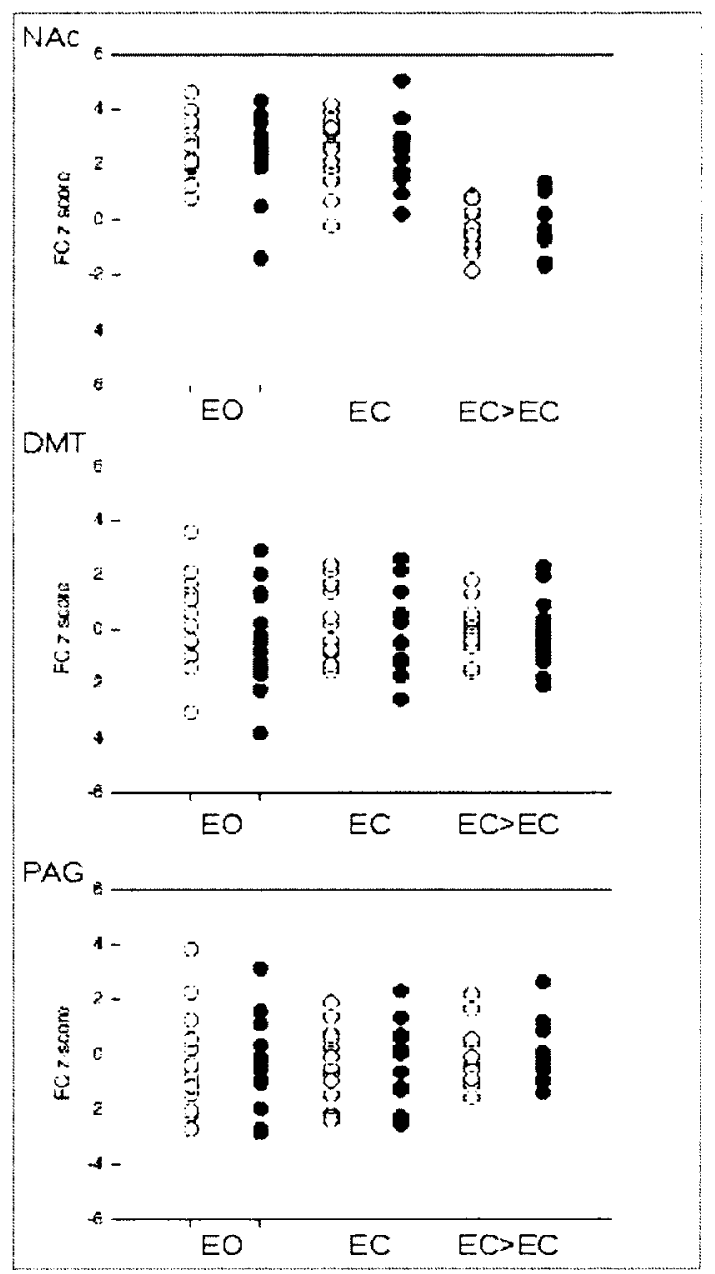

Figure A.1: Distribution of mPFC to target FC z-scores for each region. Each point represents one subject. Unfilled circles $=$ left hemisphere, filled circles $=$ right hemisphere. 


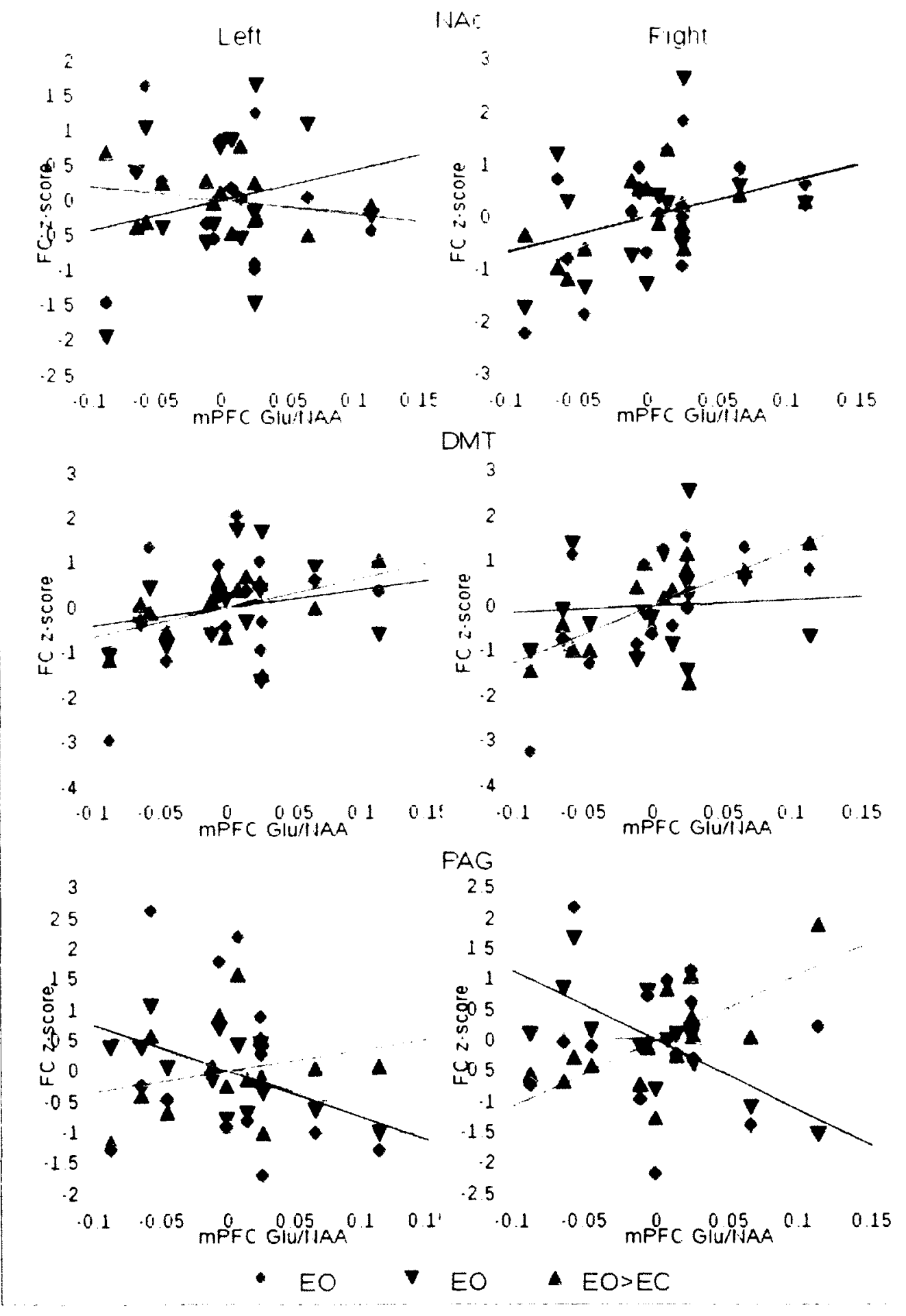

Figure A.2: Correlation plots for FC for all regions us mPFC Glu/NAA in EO, EC and EO EC conditions. 

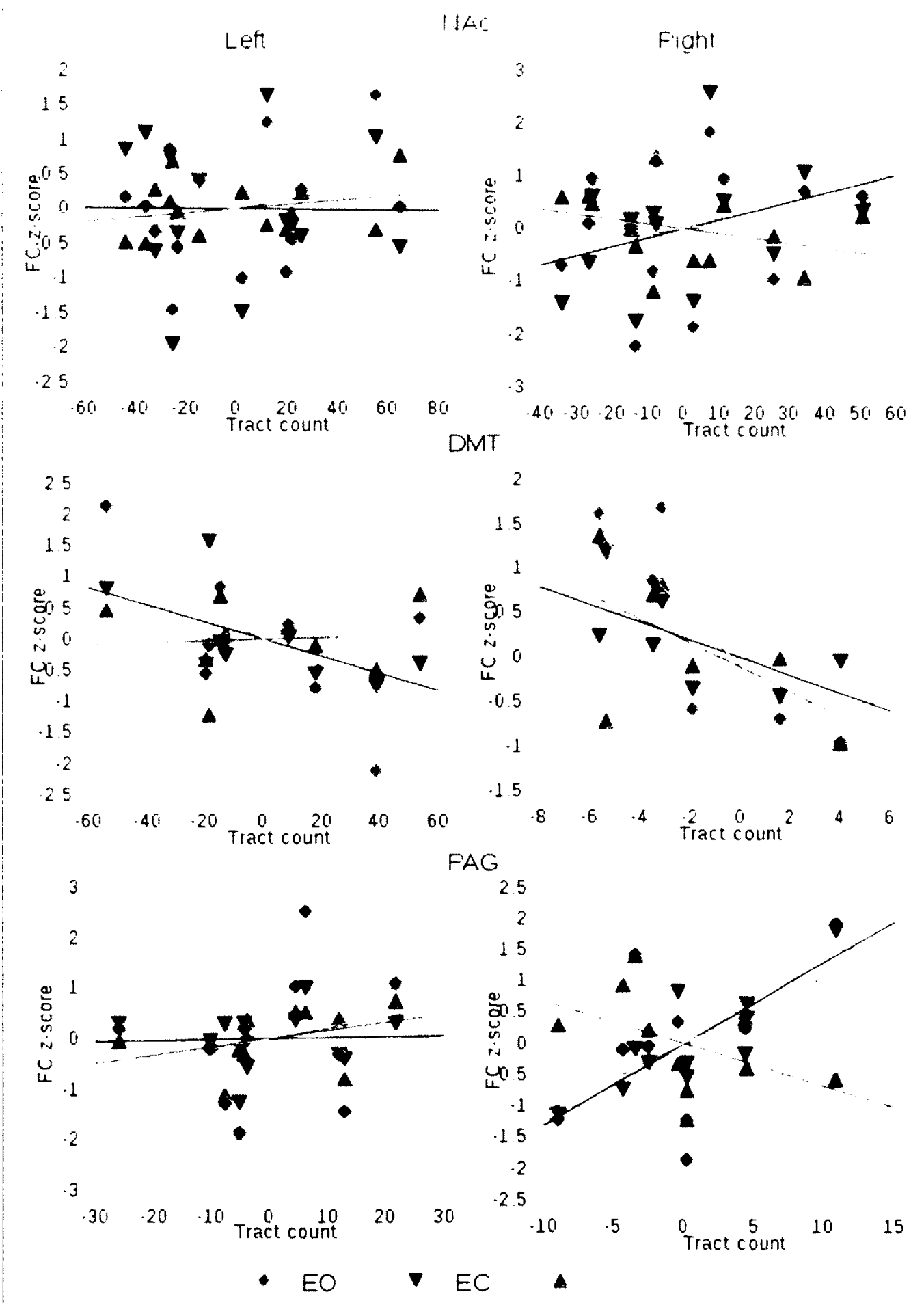

Figure A.3: Correlation plots for FC for all regions rs track count in EO. EC and EO $>\mathrm{EC}$ conditions. 


\begin{tabular}{ccccccc} 
subject & Glx/NAA & Glu/NAA & Gln/NAA & Glx/NAA & Glu/NAA & Gln/NAA \\
\hline 1 & $0.884[3]$ & $0.625[5]$ & $0.259[16]$ & $0.911[3]$ & $0.692[5]$ & $0.219[18]$ \\
2 & $0.953[3]$ & $0.736[4]$ & $0.216[18]$ & $0.952[1]$ & $0.715[2]$ & $0.237[8]$ \\
3 & $0.974[2]$ & $0.734[3]$ & $0.240[10]$ & $0.920[2]$ & $0.719[3]$ & $0.201[11]$ \\
4 & $0.927[3]$ & $0.709[4]$ & $0.218[13]$ & $0.914[2]$ & $0.734[4]$ & $0.180[17]$ \\
5 & $0.949[2]$ & $0.729[3]$ & $0.220[12]$ & $0.860[2]$ & $0.654[3]$ & $0.206[9]$ \\
6 & $1.056[2]$ & $0.762[4]$ & $0.293[11]$ & $0.727[3]$ & $0.597[3]$ & $0.130[17]$ \\
7 & $0.915[2]$ & $0.738[3]$ & $0.177[16]$ & $0.750[2]$ & $0.569[3]$ & $0.181[11]$ \\
8 & $1.065[2]$ & $0.740[3]$ & $0.325[7]$ & $0.952[3]$ & $0.675[4]$ & $0.277[12]$ \\
9 & $0.925[3]$ & $0.617[9]$ & $0.308[9]$ & $0.815[3]$ & $0.627[3]$ & $0.188[12]$ \\
10 & $1.045[3]$ & $0.815[5]$ & $0.230[20]$ & $0.829[2]$ & $0.656[3]$ & $0.173[12]$ \\
11 & $0.913[2]$ & $0.627[4]$ & $0.286[12]$ & $0.835[3]$ & $0.650[4]$ & $0.185[16]$ \\
12 & $1.086[2]$ & $0.782[3]$ & $0.304[10]$ & $0.921[2]$ & $0.716[3]$ & $0.205[11]$ \\
13 & $0.893[2]$ & $0.718[3]$ & $0.175[13]$ & $0.987[2]$ & $0.719[4]$ & $0.268[13]$ \\
& & & & & & \\
Mean (SD) & $0.968(0.07)$ & $0.718(0.06)$ & $0.250(0.05)$ & $0.875(0.08)$ & $0.671(0.05)$ & $0.204(0.04)$
\end{tabular}

Table A.1: MRS values for $\mathrm{mPFC}$ and left insula. Metabolite concentrations in relation to NAA are given. Cramér-Rao lower bounds (\%SD) for Glx, Glu, and Gln are shown in square brackets. 


\begin{tabular}{ccccc}
\multicolumn{1}{c}{$\mathrm{L}$} & \multicolumn{2}{c}{$\mathrm{R}$} \\
\hline NAC & mPFC Gln/NAA & Insula Glu/NAA & mPFC GIn/NAA & Insula Glu/NAA \\
EO & $-0.06(0.84)$ & $0.23(0.41)$ & $-0.24(0.41)$ & $0.14(0.61)$ \\
EC & $0.01(0.95)$ & $0.4(0.15)$ & $-0.09(0.74)$ & $0.4(0.15)$ \\
EO $>$ EC & $-0.25(0.39)$ & $-0.63(0.015)$ & $-0.29(0.30)$ & $-0.55(0.038)$ \\
DMT & & & & \\
EO & $-0.1(0.72)$ & $0.08(0.78)$ & $-0.07(0.82)$ & $0.08(0.79)$ \\
EC & $0.16(0.59)$ & $0.23(0.42)$ & $0.15(0.61)$ & $0.31(0.27)$ \\
EO $>$ EC & $-0.29(0.3)$ & $-0.13(0.65)$ & $0.17(0.56)$ & $-0.17(0.56)$ \\
PAG & & & & $-0.02(0.94)$ \\
EO & $0.21(0.48)$ & $0.07(0.8)$ & $0.05(0.85)$ & $0.19(0.51)$ \\
EC & $0.17(0.54)$ & $0.18(0.54)$ & $0.01(0.99)$ & $-0.15(0.6)$ \\
EO $>$ EC & $0.15(0.6)$ & $0.01(0.97)$ & $0.01(0.97)$ &
\end{tabular}

Table A.2: Regional and biochemical specificity controls. Correlations between mPFC to target FC and mPFC Gln/NAA + left insula Glu/NAA. Correlations significant at $p<0.05$ are marked in bold. $\dagger$ denotes a trend to significance. 
Appendix B

Supplementary material for Chapter 3 


\begin{tabular}{|c|c|c|c|c|}
\hline Region & & $x, y, z$ & voxels & Z-score \\
\hline \multicolumn{5}{|c|}{ Positive signal changes } \\
\hline \multirow[t]{4}{*}{ Fusiform gyrus } & $\mathrm{R}$ & $42-58-24$ & 1064 & 5.86 \\
\hline & & $18-96-18$ & & \\
\hline & $\mathrm{L}$ & $-16-94-26$ & 249 & 4.46 \\
\hline & & $-30-88-26$ & & \\
\hline \multirow{4}{*}{$\begin{array}{c}\text { Amygdala/putamen/ } \\
\text { hippocampus }\end{array}$} & $\mathrm{L}$ & $-18-18-20$ & 239 & 4.83 \\
\hline & & $-140-8$ & & \\
\hline & $\mathrm{R}$ & $22-6-16$ & 366 & 4.01 \\
\hline & & $18-16-20$ & & \\
\hline \multirow[t]{2}{*}{ Inferior frontal gyrus } & $\mathrm{R}$ & 482822 & 223 & 4.74 \\
\hline & & 38826 & & \\
\hline \multirow[t]{2}{*}{$\mathrm{sgACC} / \mathrm{SMA}$} & $\mathrm{L} / \mathrm{R}$ & -81842 & 552 & 4.16 \\
\hline & & 82044 & & \\
\hline Cerebellum & $\mathrm{L}$ & $-40-54-30$ & 238 & 4.10 \\
\hline Insula/inferior & $\mathrm{L}$ & $-34 \quad 16-2$ & 285 & 3.87 \\
\hline \multirow[t]{2}{*}{ frontal gyrus } & & $\begin{array}{lll}-48 & 18 & -4\end{array}$ & & \\
\hline & & -56160 & & \\
\hline \multicolumn{5}{|c|}{ Negative signal changes } \\
\hline \multirow[t]{2}{*}{ Cuneus/cerebellum } & $\mathrm{R}$ & $14-8818$ & 8711 & 5.31 \\
\hline & & $12-58-12$ & & \\
\hline \multirow[t]{2}{*}{ Supramarginal gyrus } & $\mathrm{R}$ & $60-5624$ & 542 & 4.58 \\
\hline & & $46-726$ & & \\
\hline \multirow[t]{2}{*}{ Superior frontal gyrus } & $\mathrm{L}$ & -342640 & 273 & 4.52 \\
\hline & $\mathrm{R}$ & 262836 & 257 & 3.65 \\
\hline Precuneus/PCC & $L / R$ & $-4-4840$ & 602 & 4.35 \\
\hline \multirow[t]{2}{*}{ Middle temporal gyrus } & $\mathrm{R}$ & $60-20-18$ & 440 & 3.86 \\
\hline & & $60-28-12$ & & \\
\hline
\end{tabular}

Table B.1: Regions of positive and negative signal changes for empathy condition (contrast [empathy $>$ view smoothed $]$ ). Coordinates are given in MNI space. (sgACC $=$ supragenual anterior cingulate cortex; SMA = supplementary motor area; $\mathrm{PCC}=$ posterior cingulate cortex) 


\begin{tabular}{|c|c|c|c|c|}
\hline Region & & $x, y, z$ & voxels & Z-score \\
\hline \multirow[t]{3}{*}{$\mathrm{PCC} /$ precuneus } & $\mathrm{L} / \mathrm{R}$ & $10-6212$ & 3503 & 5.61 \\
\hline & & $-6-7214$ & & \\
\hline & & $\begin{array}{lll}0 & -86 & 12\end{array}$ & & \\
\hline \multirow[t]{3}{*}{$\mathrm{pgACC} / \mathrm{MPFC}$} & $\mathrm{L} / \mathrm{R}$ & -6408 & 2045 & 5.50 \\
\hline & & $1254-10$ & & \\
\hline & & 124816 & & \\
\hline \multirow[t]{4}{*}{ Middle temporal gyrus } & L & $-42-8024$ & 390 & 5.12 \\
\hline & & $\begin{array}{llll}-52 & -66 & 18\end{array}$ & & \\
\hline & $\mathbf{R}$ & $\begin{array}{lll}48 & -74 & 18\end{array}$ & 393 & 5.02 \\
\hline & & $64-16-16$ & & \\
\hline \multirow[t]{2}{*}{ Middle frontal gyrus } & L & -282842 & 290 & 5.11 \\
\hline & $\mathbf{R}$ & 263042 & & \\
\hline Superior temporal gyrus & $\mathbf{R}$ & $44-184$ & 432 & 4.78 \\
\hline /posterior insula & & $62-106$ & & \\
\hline
\end{tabular}

Table B.2: Regions showing empathy task induced deactivation from rest (contrast [fixation > empathy]). Coordinates are given in MNI space. (MPFC = medial prefrontal cortex) 


\begin{tabular}{|c|c|c|c|c|}
\hline Region & & $\mathrm{x}, \mathrm{y}, \mathrm{z}$ & voxels & Z-score \\
\hline \multirow[t]{2}{*}{ Supramarginal gyrus } & $\mathrm{R}$ & $50-46 \quad 38$ & 966 & 4.84 \\
\hline & & $42-5038$ & & \\
\hline \multirow{4}{*}{$\begin{array}{c}\text { Thalamus/ventral } \\
\text { striatum }\end{array}$} & $\mathrm{L}$ & $-12-142$ & 121 & 4.47 \\
\hline & & $-6-206$ & & \\
\hline & $\mathbf{R}$ & $10-86$ & 460 & 4.35 \\
\hline & & $16-26$ & & \\
\hline \multirow[t]{2}{*}{$\mathrm{PCC}$} & $\mathrm{L} / \mathrm{R}$ & $-12-4232$ & 2437 & 4.42 \\
\hline & & $2-3222$ & & \\
\hline \multirow{2}{*}{$\begin{array}{c}\text { Hippocampus/ } \\
\text { amygdala }\end{array}$} & $\mathrm{R}$ & $22-30-6$ & 1029 & 4.34 \\
\hline & & $44-268$ & & \\
\hline \multirow[t]{2}{*}{$\operatorname{sgACC} / \mathrm{MCC}$} & $L / R$ & 02232 & 251 & 3.81 \\
\hline & & -41226 & & \\
\hline Fusiform gyrus & $\mathrm{L}$ & $-56-56-18$ & 243 & 3.80 \\
\hline Superior frontal gyrus & $\mathbf{R}$ & 361056 & 175 & 3.79 \\
\hline Superior temporal gyrus & L & $-54-3414$ & 194 & 3.57 \\
\hline
\end{tabular}

Table B.3: Regions displaying positive functional connectivity with pgACC MRS region during empathy task (pgACC seed contrast - [fixation > empathy]). No regions of negative functional connectivity were observed. Coordinates are given in MNI space. $(\mathrm{MCC}=$ mid cingulate cortex)

\begin{tabular}{|c|c|c|c|c|}
\hline Region & & $x, y, z$ & voxels & Z-score \\
\hline \multirow[t]{2}{*}{ Angular gyrus } & L & $-42-6238$ & 271 & 4.35 \\
\hline & & $-36-5424$ & & \\
\hline \multirow[t]{2}{*}{$\mathrm{PCC} / \mathrm{MCC}$} & $\mathrm{L} / \mathrm{R}$ & -6022 & 375 & 4.08 \\
\hline & & $8-488$ & & \\
\hline \multirow[t]{2}{*}{ Cuneus } & $\mathrm{R}$ & $6-8240$ & 223 & 3.79 \\
\hline & & $0-7626$ & & \\
\hline
\end{tabular}

Table B.4: Regions displaying positive functional connectivity with sgACC MRS region during empathy task (sgACC seed contrast - [empathy > view smoothed]). No regions of negative functional connectivity were observed. Coordinates are given in MNI space. 


\begin{tabular}{ccccc} 
Region & & $\mathrm{x}, \mathrm{y}, \mathrm{x}$ & voxels & Z-score \\
\hline Superior frontal gyrus & $\mathrm{R}$ & 244426 & 96 & 4.86 \\
& & 264634 & & \\
Fusiform gyrus & $\mathrm{R}$ & $44-60-16$ & 79 & 3.61 \\
& & $-408-8$ & & \\
Insula/middle temporal gyrus & $\mathrm{L}$ & $-506-18$ & 52 & 3.18
\end{tabular}

Table B.5: Regions showing correlation between BOLD response during empathy task (contrast lempathy $>$ view smoothed!) and $\mathrm{Glx} / \mathrm{Cr}$ levels in $\mathrm{sgACC}$. Coordinates are given in MNI space. 
Appendix C

Supplementary material for Chapter 7 

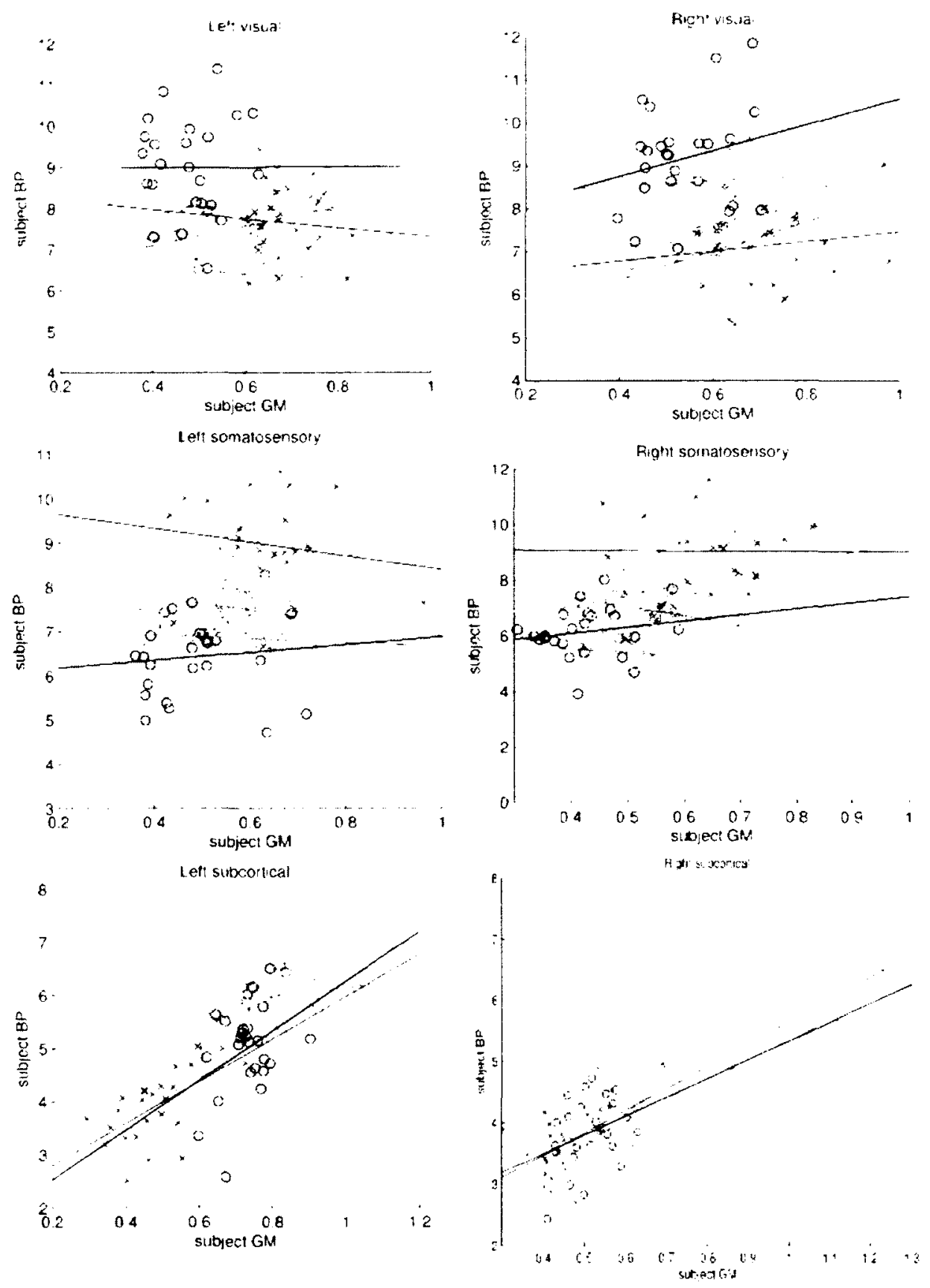

Figure C.1: (A) Scatter plots for cach ROI with lincar fits. ROIs are grouped into regions that are close to each other in the brain. The plots illustrate that a relationship between $\mathrm{BP}_{\mathrm{ND}}$ and GMI can be seen in some regions, but that in others there is not relationship across subjects. 

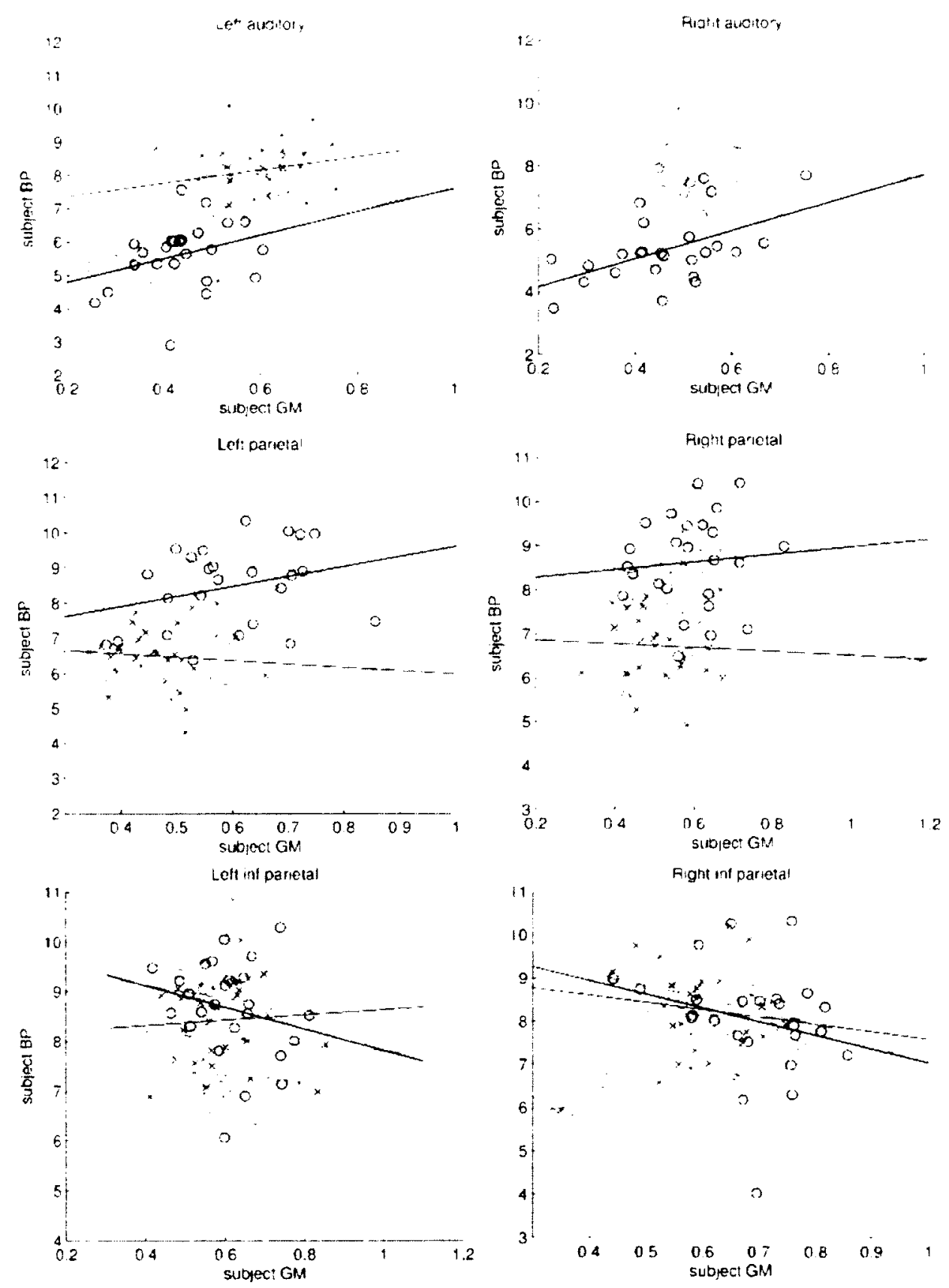

Figure C.2: (B) Scatter plots for each ROI with linear fits. ROIs are grouped into regions that are close to each other in the brain. The plots illustrate that a relationship between BPND and GM can be seen in some regions, but that in others there is not relationship across subjects. 


\section{Appendix D}

Supplementary material for Chapter 9 
(A)

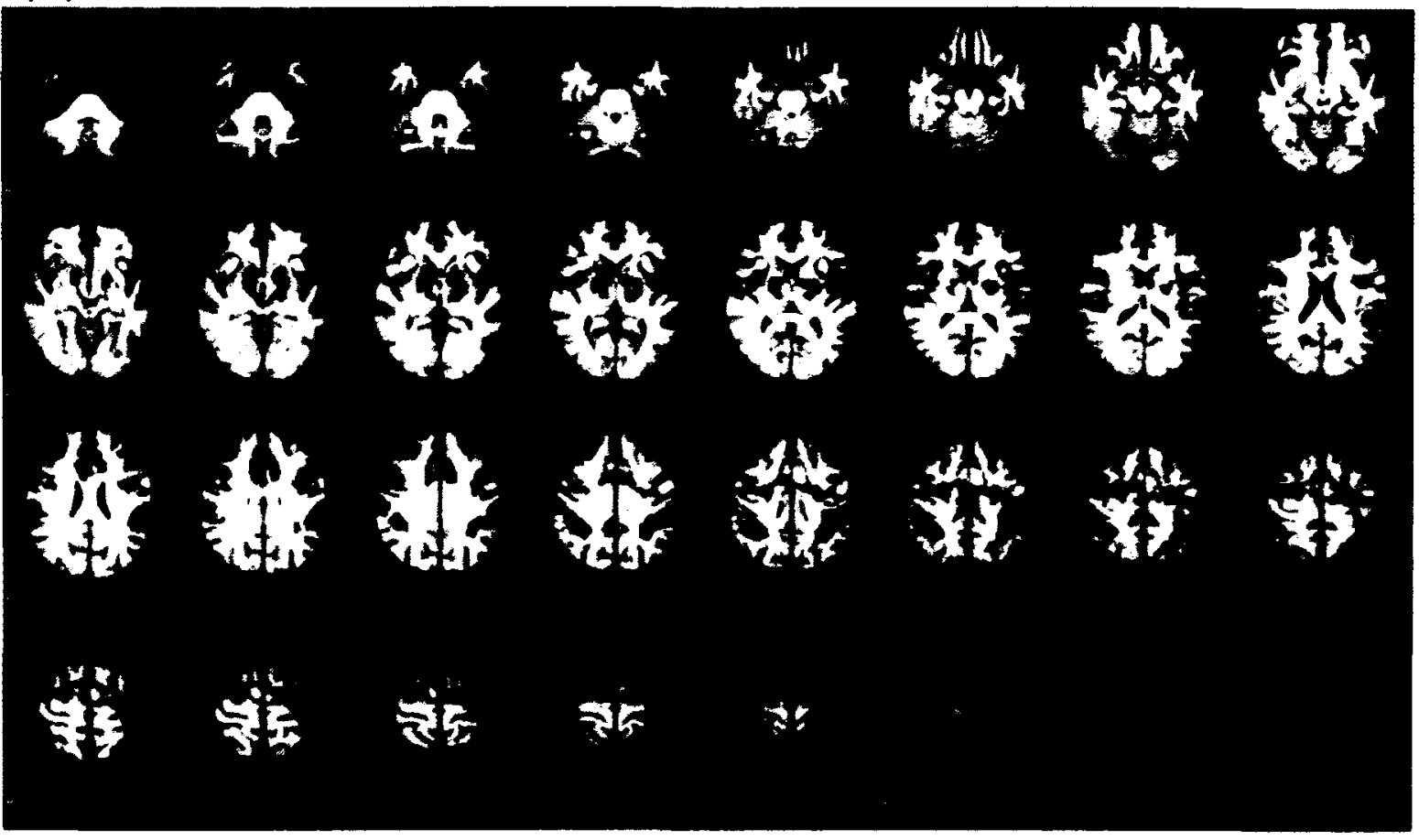

(B)

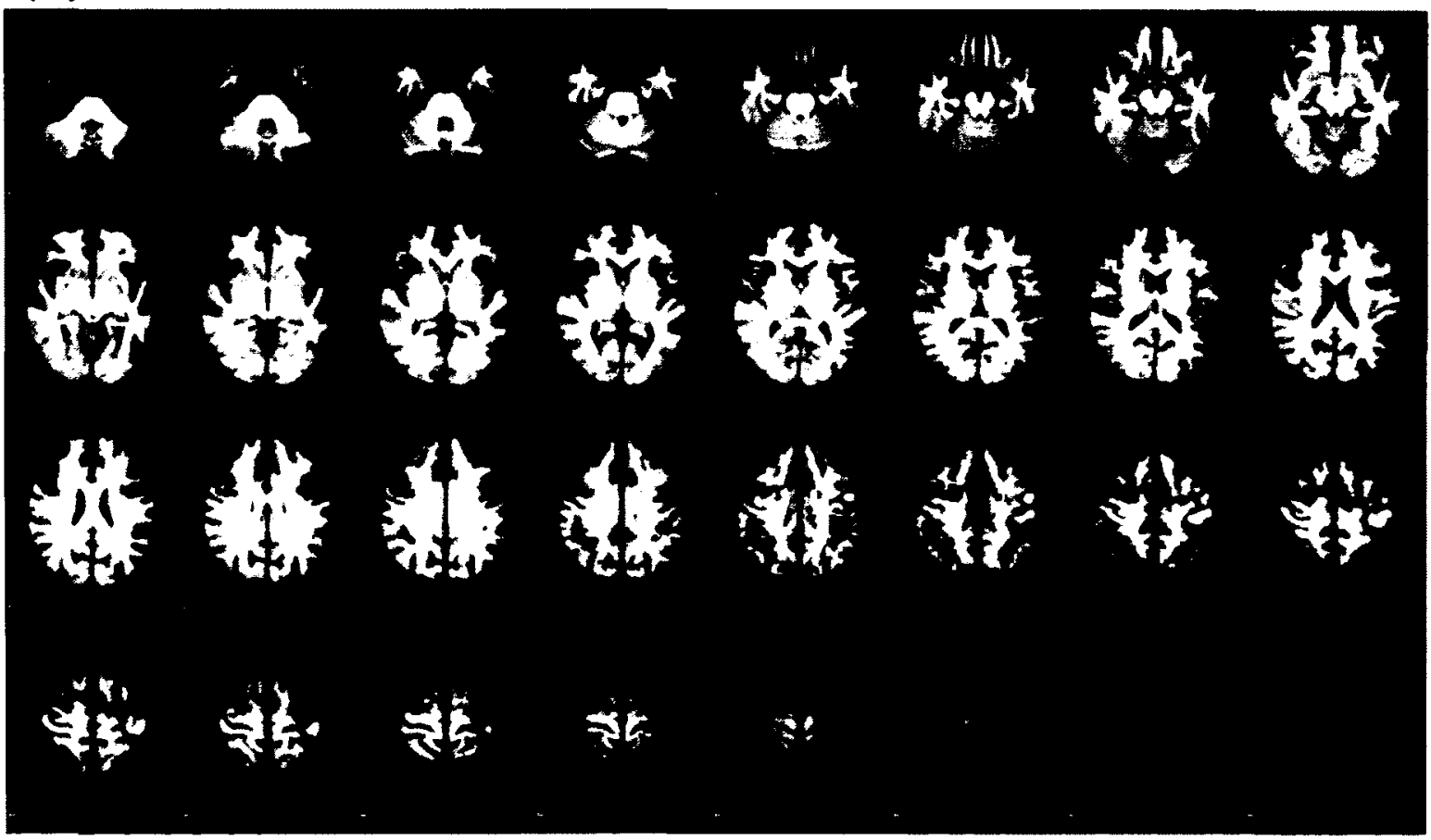

Figure D.1: Group image for (A) contrast [int $>$ fix], and (B) contrast [ext $\therefore$ fix] ( $p_{\text {FwE }}$ 0.05). Results displayed on group mean anatomical image. 


\begin{tabular}{|c|c|c|c|}
\hline Region & & $x, y, z$ & Z-score \\
\hline \multirow{7}{*}{$\begin{array}{l}\text { Insula/operculum/ } \\
\text { precentral gyrus }\end{array}$} & $\mathrm{R}$ & 3446 & 6.23 \\
\hline & & 5446 & \\
\hline & & 56210 & \\
\hline & $\mathrm{L}$ & $-38-26$ & 5.95 \\
\hline & & -5224 & \\
\hline & & -62820 & \\
\hline & & -28286 & \\
\hline SMA & $\mathrm{L} / \mathrm{R}$ & $10-864$ & 5.39 \\
\hline \multirow[t]{4}{*}{ SMG } & $\mathrm{L}$ & $-62-3230$ & 5.86 \\
\hline & & $-52-2626$ & \\
\hline & $\mathrm{R}$ & $54-2628$ & 5.22 \\
\hline & & $60-2234$ & \\
\hline \multirow[t]{6}{*}{ Cerebellum } & $\mathrm{R}$ & $32-58-28$ & 5.39 \\
\hline & & $26-68-24$ & \\
\hline & & $18-72-52$ & \\
\hline & $\mathrm{L}$ & $-32-58-26$ & 4.99 \\
\hline & & $-28-64-22$ & \\
\hline & & $-32-54-56$ & \\
\hline \multirow{6}{*}{$\begin{array}{l}\text { Mid frontal gyrus/ } \\
\text { inf. frontal gyrus/ } \\
\text { frontal pole }\end{array}$} & $\mathrm{L}$ & -38368 & 4.55 \\
\hline & & -403818 & \\
\hline & & -344232 & \\
\hline & $\mathrm{R}$ & 264016 & 4.39 \\
\hline & & 303820 & \\
\hline & & 324626 & \\
\hline \multirow{5}{*}{$\begin{array}{l}\text { Putamen/ } \\
\text { pallidum }\end{array}$} & L & $-188-8$ & 4.11 \\
\hline & & $-2618-6$ & \\
\hline & & -22146 & \\
\hline & $\mathrm{R}$ & $28-186$ & 4.25 \\
\hline & & $24-100$ & \\
\hline \multirow{3}{*}{$\begin{array}{l}\text { Post. cent. gyrus/ } \\
\text { SPL }\end{array}$} & $\mathrm{R}$ & $34-3440$ & 3.88 \\
\hline & & $32-3846$ & \\
\hline & L & $-30-4632$ & 3.59 \\
\hline \multirow[t]{2}{*}{ Precent. gyrus } & $\mathrm{L}$ & $-36-650$ & 3.55 \\
\hline & $\mathrm{R}$ & $46-454$ & 3.51 \\
\hline \multirow[t]{2}{*}{ Caudate } & $\mathrm{R}$ & 14142 & 3.57 \\
\hline & $\mathrm{L}$ & -14164 & 2.93 \\
\hline
\end{tabular}

Table D.1: Activations for contrast [int > ext]. 


\begin{tabular}{|c|c|c|c|}
\hline Region & & $\mathrm{x}, \mathrm{y}, \mathrm{z}$ & Z-score \\
\hline \multirow{2}{*}{$\begin{array}{l}\text { Mid/sup. temporal } \\
\text { gyrus/Heschl's }\end{array}$} & $\mathrm{R}$ & $72-300$ & 5.03 \\
\hline & & $62-240$ & \\
\hline \multirow[t]{2}{*}{ gyrus } & $L$ & $-44-22-4$ & 4.73 \\
\hline & & $-36-348$ & \\
\hline \multirow[t]{4}{*}{ Lat. occ. cortex } & $\mathrm{R}$ & $40-70 \quad 42$ & 4.58 \\
\hline & & $42-6644$ & \\
\hline & $\mathrm{L}$ & $-24-7446$ & 4.01 \\
\hline & & $-20-7836$ & \\
\hline \multirow[t]{2}{*}{$1^{\circ}$ motor cortex } & $\mathrm{L} / \mathrm{R}$ & $-6-3456$ & 4.7 \\
\hline & & $6-2860$ & \\
\hline \multirow[t]{3}{*}{ Visual cortex } & L & $-28-9218$ & 5.57 \\
\hline & & $-34-92-4$ & \\
\hline & $\mathrm{R}$ & $32-960$ & 3.91 \\
\hline \multirow[t]{3}{*}{ Precuneus/ICC } & $\mathrm{R}$ & $4-7224$ & 4.47 \\
\hline & & $20-606$ & \\
\hline & & $-18-604$ & \\
\hline \multirow{2}{*}{$\begin{array}{l}\text { Sup. occipital/ } \\
\text { cuneus }\end{array}$} & $\mathrm{L}$ & $-14-8426$ & 4.95 \\
\hline & & $-14-7618$ & \\
\hline Precent. gyrus & $\mathrm{R}$ & $30-2654$ & 4.24 \\
\hline \multirow[t]{2}{*}{ Angular gyrus } & & $56-5234$ & \\
\hline & & $46-5214$ & \\
\hline $\mathrm{PCC}$ & $\mathrm{R} / \mathrm{L}$ & $8-4230$ & 4.11 \\
\hline \multirow[t]{2}{*}{ Mid/sup frontal gyrus } & $\mathrm{R}$ & 282852 & 4.39 \\
\hline & & 401830 & \\
\hline \multirow[t]{2}{*}{ Frontal pole } & $\mathrm{L} / \mathrm{R}$ & 66026 & 4.03 \\
\hline & & 125420 & \\
\hline Parrahipp. gyrus & $\mathrm{R}$ & $28-26-28$ & 3.86 \\
\hline \multirow[t]{2}{*}{ Cerebellum } & $\mathrm{L}$ & $-18-50-28$ & 4.1 \\
\hline & & $-6-50-16$ & \\
\hline \multirow[t]{2}{*}{$\mathrm{mPFC} / \mathrm{sgACC}$} & $\mathrm{L} / \mathrm{R}$ & $242-10$ & 3.54 \\
\hline & & $626-16$ & \\
\hline
\end{tabular}

Table D.2: Activations for contrast [ext $>$ int]. 


\begin{tabular}{|c|c|c|c|}
\hline Region & & $\mathrm{x}, \mathrm{y}, \mathrm{z}$ & Z-score \\
\hline \multirow{4}{*}{$\begin{array}{c}\text { Precentral gyrus/ } \\
\text { ant. insula/ } \\
\text { operculum }\end{array}$} & $\mathrm{L}$ & -60822 & 6.93 \\
\hline & & $-3420-4$ & \\
\hline & $\mathrm{R}$ & 30202 & 6.42 \\
\hline & & 36244 & \\
\hline \multirow[t]{2}{*}{$\mathrm{ACC} / \mathrm{SMA}$} & $\mathrm{L} / \mathrm{R}$ & -10842 & 6.25 \\
\hline & & $-4-450$ & \\
\hline \multirow[t]{2}{*}{ Frontal pole } & $\mathrm{R}$ & 344222 & 4.89 \\
\hline & & 363622 & \\
\hline Supramarginal & $\mathrm{R}$ & $48-3438$ & 5.2 \\
\hline \multirow[t]{3}{*}{ gyrus } & & $46-4044$ & \\
\hline & $\mathrm{L}$ & $-46-3828$ & 4.57 \\
\hline & & $-46-4230$ & \\
\hline \multirow[t]{4}{*}{ Cerebellum } & $\mathrm{R}$ & $32-58-30$ & 5.91 \\
\hline & & $10-74-50$ & \\
\hline & $\mathrm{L}$ & $-26-64-26$ & 5.6 \\
\hline & & $-24-64-56$ & \\
\hline \multirow[t]{3}{*}{ Postcent. Gyrus } & $\mathrm{L}$ & $-56-2224$ & 4.78 \\
\hline & & $-60-2018$ & \\
\hline & $\mathrm{R}$ & $52-1826$ & 4.48 \\
\hline \multirow[t]{3}{*}{ Putamen/Pallidum } & $\mathrm{R}$ & $1610-4$ & 4.93 \\
\hline & $\mathrm{L}$ & $-22-6 \quad 12$ & 4.29 \\
\hline & & $-126-2$ & \\
\hline \multirow[t]{2}{*}{ Thalamus } & $\mathrm{L}$ & $-16-140$ & 4.58 \\
\hline & $\mathrm{R}$ & $12-10-2$ & 4.18 \\
\hline \multirow[t]{2}{*}{ Brainstem } & $\mathrm{L} / \mathrm{R}$ & $6-22-8$ & 3.31 \\
\hline & & $-6-20-10$ & \\
\hline
\end{tabular}

Table D.3: Activations for contrast [int $>$ fix]. 


\begin{tabular}{|c|c|c|c|}
\hline Region & & $x, y, z$ & Z-score \\
\hline \multirow{3}{*}{$\begin{array}{c}\text { Post } / \text { pre central } \\
\text { gyrus }\end{array}$} & $\mathrm{L}$ & $-48-2050$ & 5.95 \\
\hline & & $-50-244$ & \\
\hline & $\mathrm{R}$ & 48828 & 4.58 \\
\hline \multirow{4}{*}{$\begin{array}{l}\text { Ant. Insula/ } \\
\text { operculum }\end{array}$} & $\mathrm{R}$ & 461410 & 4.88 \\
\hline & & $4620-2$ & \\
\hline & $\mathrm{L}$ & -30300 & 4.18 \\
\hline & & -26242 & \\
\hline \multirow[t]{4}{*}{$\mathrm{ACC} / \mathrm{SMA}$} & $\mathrm{L} / \mathrm{R}$ & 41450 & 4.65 \\
\hline & & 18448 & \\
\hline & & $-12-450$ & \\
\hline & & -101838 & \\
\hline \multirow[t]{4}{*}{ Supramarginal gyrus } & $\mathrm{R}$ & $50-3640$ & 4.39 \\
\hline & & $54-4056$ & \\
\hline & $\mathrm{L}$ & $-48-3640$ & 3.76 \\
\hline & & $-46-4414$ & \\
\hline \multirow[t]{2}{*}{ Frontal pole } & $\mathrm{R}$ & $4848 \quad 14$ & 4.21 \\
\hline & & 483626 & \\
\hline \multirow[t]{2}{*}{ Sup. temporal gyrus } & $\mathrm{L}$ & $-64-4012$ & 4.19 \\
\hline & & $-58-4612$ & \\
\hline \multirow[t]{2}{*}{ Angular gyrus } & $\mathrm{R}$ & $36-5436$ & 4.39 \\
\hline & & $54-5052$ & \\
\hline \multirow[t]{4}{*}{ Lat. occ. cortex } & $\mathrm{R}$ & $24-6054$ & 4.28 \\
\hline & & $30-6854$ & \\
\hline & $\mathrm{L}$ & $-22-6456$ & 3.89 \\
\hline & & $-28-5854$ & \\
\hline Sup. frontal gyrus & $\mathrm{L}$ & $-24-458$ & 4.66 \\
\hline \multirow[t]{3}{*}{ Cerebellum } & $\mathrm{R}$ & $16-52-24$ & 4.44 \\
\hline & & $28-54-30$ & \\
\hline & L & $-28-60-34$ & 3.6 \\
\hline \multirow[t]{2}{*}{ Brainstem } & $\mathrm{L} / \mathrm{R}$ & $-6-22-8$ & 3.6 \\
\hline & & $6-24-8$ & \\
\hline
\end{tabular}

Table D.4: Activations for contrast [ext $>$ fix]. 


\begin{tabular}{|c|c|c|c|c|c|c|c|c|c|c|}
\hline & Region & \multicolumn{3}{|c|}{ Coefficient (C.I.) } & $\mathrm{Z}$ & Region & \multicolumn{3}{|c|}{ Coefficient (C.I.) } & $\mathrm{Z}$ \\
\hline & \multicolumn{5}{|c|}{ int $>$ fix $\mid$} & \multicolumn{5}{|c|}{$\mid$ ext $>$ fix $\mid$} \\
\hline & L pars.triang. & 0.33 & $(0.29$ & $0.36)$ & 3.9 & L ventralDC & -0.20 & $(-0.24$ & $-0.15)$ & -2.6 \\
\hline \multirow{11}{*}{ t. } & R sup.front. & -0.23 & $(-0.27$ & $-0.19)$ & -2.8 & L lat.occip. & -0.14 & $(-0.21$ & $-0.12)$ & -2.0 \\
\hline & L lat.occip. & -0.21 & $(-0.25$ & $-0.18)$ & -2.6 & L inf.par. & -0.13 & $(-0.16$ & $-0.10)$ & -1.7 \\
\hline & R precuneus & -0.20 & $(-0.21$ & $-0.19)$ & -2.5 & L cMFG & 0.12 & $(0.10$ & $0.13)$ & 1.5 \\
\hline & $\mathrm{L} \mathrm{mOFC}$ & -0.20 & $(-0.21$ & $-0.18)$ & -2.4 & L thalamus & 0.12 & $(0.08$ & $0.13)$ & 1.5 \\
\hline & L pars.operc. & 0.20 & $(0.15$ & $0.22)$ & 2.4 & L cACC & -0.11 & $(-0.13$ & $-0.08)$ & -1.5 \\
\hline & L rMFG & -0.19 & $(-0.21$ & $-0.14)$ & -2.3 & brainstem & 0.10 & $(0.07$ & $0.13)$ & 1.3 \\
\hline & $\mathrm{R} C A C C$ & 0.16 & $(0.14$ & $0.21)$ & 1.9 & & & & & \\
\hline & L sup.parietal & 0.14 & $(0.10$ & $0.20)$ & 1.6 & & & & & \\
\hline & $\mathrm{L}$ ventralDC & -0.11 & $(-0.13$ & $-0.08)$ & -1.4 & & & & & \\
\hline & $\mathrm{L} c \mathrm{ACC}$ & -0.11 & $(-0.14$ & $-0.10)$ & -1.4 & & & & & \\
\hline & L sup.front. & 0.11 & $(0.09$ & $0.13)$ & 1.3 & & & & & \\
\hline \multirow{10}{*}{$\begin{array}{c}\text { L. post. } \\
\text { insula }\end{array}$} & L trans.temp. & -0.23 & $(-0.26$ & $-0.21)$ & -3.6 & $\mathrm{~L}$ thalamus & 0.16 & $(0.13$ & $0.20)$ & 2.5 \\
\hline & R. PCC & 0.18 & $(0.16$ & $0.2)$ & 2.7 & L $\mathrm{rMFG}$ & 0.13 & $(0.08$ & $0.19)$ & 2.0 \\
\hline & R sup.front. & 0.16 & $(0.13$ & $0.20)$ & 2.6 & $\mathrm{~L}$ precuneus & -0.12 & $(-0.18$ & $-0.09)$ & -1.8 \\
\hline & $\mathrm{L}$ postcentral & -0.15 & $(-0.17$ & $-0.14)$ & -2.4 & L amygdala & -0.10 & $(-0.13$ & $-0.09)$ & -1.6 \\
\hline & L precentral & 0.15 & $(0.13$ & $0.17)$ & 2.5 & $\mathrm{R}$ putamen & -0.10 & $(-0.12$ & $-0.08)$ & -1.5 \\
\hline & R precentral & 0.13 & $(0.12$ & $0.15)$ & 2.1 & L sup.front. & 0.10 & $(0.04$ & $0.12)$ & 1.5 \\
\hline & $\mathrm{R}$ cMFG & 0.13 & $(0.11$ & $0.15)$ & 2.0 & R. pallidum & 0.10 & $(0.09$ & $0.12)$ & 1.5 \\
\hline & L PCC & -0.12 & $(-0.14$ & $-0.10)$ & -2.0 & L inf.par. & -0.09 & $(-0.12$ & $-0.05)$ & -1.4 \\
\hline & L caudate & -0.10 & $(-0.12$ & $-0.07)$ & -1.5 & & & & & \\
\hline & $\mathrm{L}$ precuneus & -0.10 & $(-0.12$ & $-0.07)$ & -1.5 & & & & & \\
\hline
\end{tabular}

Table D.5: Coefficients with $95 \%$ confidence intervals and z-scores for the model prediciting $[$ int $>$ fix $\mid$ and $\mid$ ext $>$ fix $\mid$ functional responses in the left anterior and posterior insula. 


\begin{tabular}{|c|c|c|c|c|c|c|c|c|c|c|}
\hline & Region & $\mathrm{Coe}$ & fficient & C.I.) & $\mathrm{Z}$ & Region & Coe & ficient & C.I.) & $\mathrm{Z}$ \\
\hline & & & int $>$ fix & & & & & $e x t>f$ & & \\
\hline & R pars.triang. & 0.30 & $(0.25$ & $0.32)$ & 3.8 & R pars.triang. & 0.35 & $(0.32$ & $0.38)$ & 3.6 \\
\hline & R sup.parietal & 0.28 & $(0.22$ & $0.34)$ & 3.5 & $\mathrm{R}$ precentral & -0.20 & $(-0.22$ & $-0.18)$ & -2.1 \\
\hline & R pars.operc. & 0.17 & $(0.15$ & $0.20)$ & 2.2 & R IOFC & 0.20 & $(0.18$ & $0.23)$ & 2.0 \\
\hline & R sup.temp. & -0.17 & $(-0.19$ & $-0.16)$ & -2.1 & L sup.front. & 0.19 & $(0.15$ & $0.22)$ & 2.0 \\
\hline & L sup.front. & 0.17 & $(0.14$ & $0.19)$ & 2.1 & $\mathrm{R}$ inf.par. & -0.17 & $(-0.24$ & $-0.12)$ & -1.8 \\
\hline R. ant. & $R$ ventralDC & -0.17 & $(-0.19$ & $-0.14)$ & -2.1 & R amygdala & -0.15 & $(-0.18$ & $-0.12)$ & -1.5 \\
\hline insula & R sup.front. & -0.16 & $(-0.23$ & $-0.10)$ & -2.0 & R putamen & 0.14 & $(0.11$ & $0.18)$ & 1.4 \\
\hline & $\mathrm{R} \mathrm{mOFC}$ & -0.15 & $(-0.18$ & $-0.11)$ & -1.9 & & & & & \\
\hline & R lat.occip. & -0.11 & $(-0.19$ & $-0.04)$ & -1.4 & & & & & \\
\hline & R peri.calc. & -0.11 & $(-0.13$ & $-0.05)$ & -1.4 & & & & & \\
\hline & $\mathrm{R} 10 \mathrm{FC}$ & 0.11 & $(0.09$ & $0.12)$ & 1.4 & & & & & \\
\hline & R putamen & 0.10 & $(0.06$ & $0.14)$ & 1.3 & & & & & \\
\hline & $\mathrm{R}$ frontalpole & -0.10 & $(-0.13$ & $-0.09)$ & -1.3 & & & & & \\
\hline & brainstem & -0.28 & $(-0.33$ & $-0.23)$ & -3.4 & R inf.temp. & -0.22 & $(-0.28$ & $-0.16)$ & -2.9 \\
\hline & L precuneus & 0.21 & $(0.11$ & $0.27)$ & 2.5 & $\mathrm{R}$ thalamus & 0.19 & $(0.17$ & $0.22)$ & 2.5 \\
\hline & R sup.front. & -0.18 & $(-0.20$ & $-0.16)$ & -2.2 & $\mathrm{R}$ inf.par. & 0.17 & $(0.12$ & $0.25)$ & 2.3 \\
\hline & $L$ ventralDC & -0.17 & $(-0.19$ & $-0.14)$ & -2.0 & $R$ ventralDC & -0.17 & $(-0.23$ & $-0.12)$ & -2.2 \\
\hline & $\mathrm{R}$ precuneus & -0.17 & $(-0.19$ & $-0.12)$ & -2.0 & L sup.parietal & 0.16 & $(0.13$ & $0.20)$ & 2.1 \\
\hline & $\mathrm{R}$ pallidum & 0.16 & $(0.13$ & 0.18 ) & 1.2 & $\mathrm{~L}$ ventralDC & -0.16 & $(-0.24$ & $-0.11)$ & -2.0 \\
\hline & R SMG & -0.16 & $(-0.19$ & $-0.15)$ & -1.9 & $\mathrm{~L} \mathrm{PCC}$ & -0.14 & $(-0.19$ & $-0.10)$ & -1.9 \\
\hline R. post. & $\mathrm{R}$ thalamus & 0.15 & $(0.13$ & $0.17)$ & 1.8 & $\mathrm{R}$ pallidum & 0.13 & $(0.10$ & $0.16)$ & 1.7 \\
\hline insula & $\mathbf{R}$ mid.temp. & 0.15 & $(0.13$ & $0.17)$ & 1.8 & L caudate & -0.13 & $(-0.15$ & $-0.09)$ & -1.6 \\
\hline & L sup.parietal & 0.14 & $(0.11$ & $0.17)$ & 1.7 & L inf.par. & -0.12 & $(-0.16$ & $-0.08)$ & -1.6 \\
\hline & R postcentral & -0.15 & $(-0.15$ & $-0.13)$ & -1.7 & L thalamus & 0.10 & $(0.07$ & $0.12)$ & 1.3 \\
\hline & $\mathrm{R}$ precentral & 0.13 & $(0.11$ & $0.15)$ & 1.6 & & & & & \\
\hline & $\mathrm{R}$ trans.temp. & -0.13 & $(-0.14$ & $-0.12)$ & -1.5 & & & & & \\
\hline & L thalamus & 0.12 & $(0.10$ & $0.14)$ & 1.5 & & & & & \\
\hline & R pars.operc. & 0.12 & $(0.11$ & $0.13)$ & 1.4 & & & & & \\
\hline & $\mathrm{R}$ inf.temp. & -0.11 & $(-0.14$ & $-0.07)$ & -1.3 & & & & & \\
\hline
\end{tabular}

Table D.6: Coefficients with $95 \%$ confidence intervals and z-scores for the model prediciting $[$ int $>$ fix $\mid$ and $\mid$ ext $>$ fix $\mid$ functional responses in the right anterior and posterior insula. 
Appendix $E$

Supplementary material for Chapter $\mathbf{1 0}$ 
These questions ask about some of your experiences growing up as a child and a teenager. Athough these questions are of a personal nature, plaase try to anewer as honestly as you can. For ench quastion, circle the dot under the response that best describes how you feel. If you wish to chenge your reaponse, put an $X$ through it and circle your new choice, as shown in the example to the right.

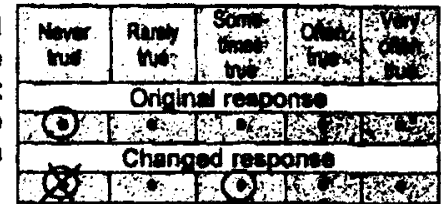

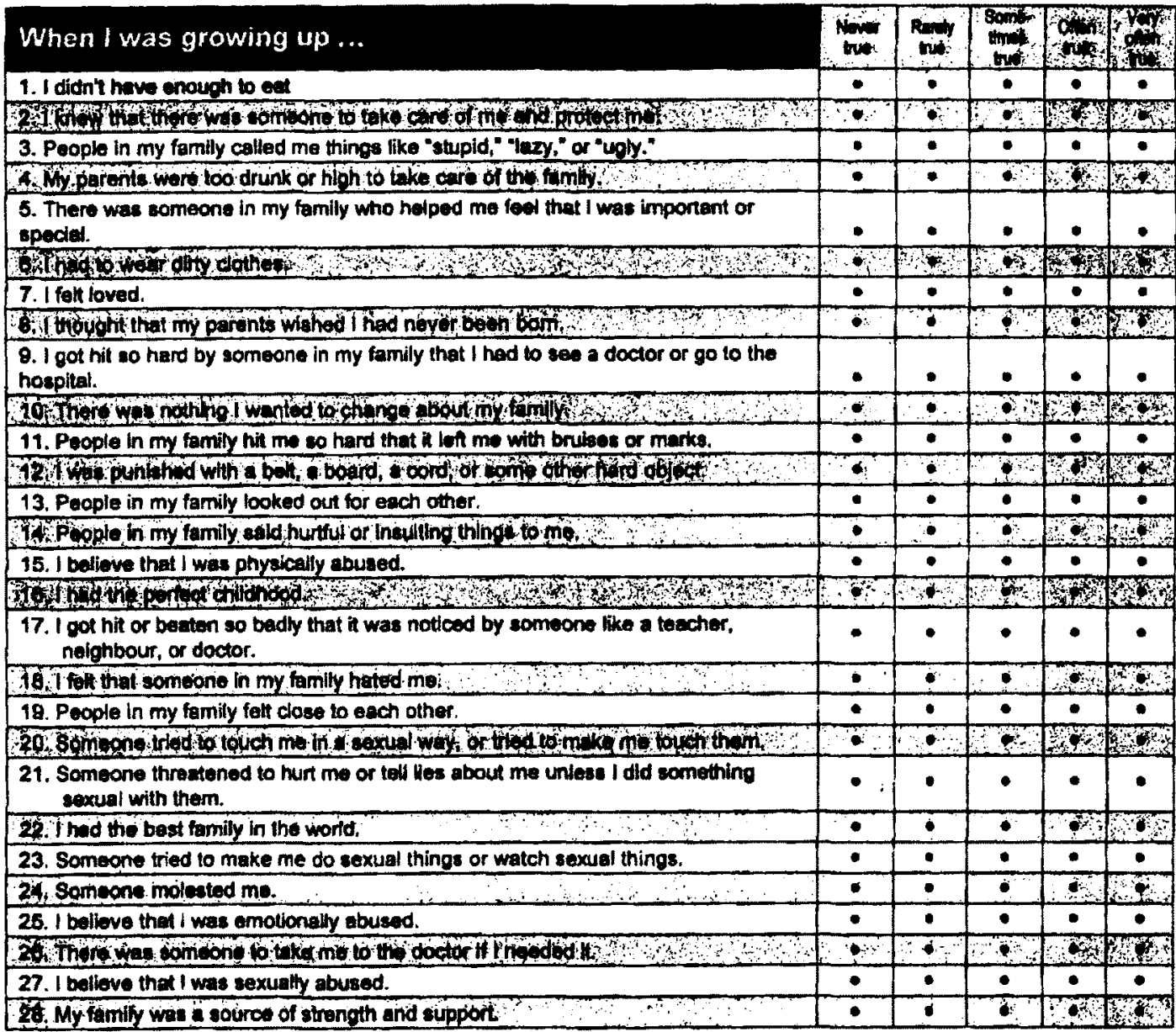

Figure E.1: Childhood trauma questionnaire. 

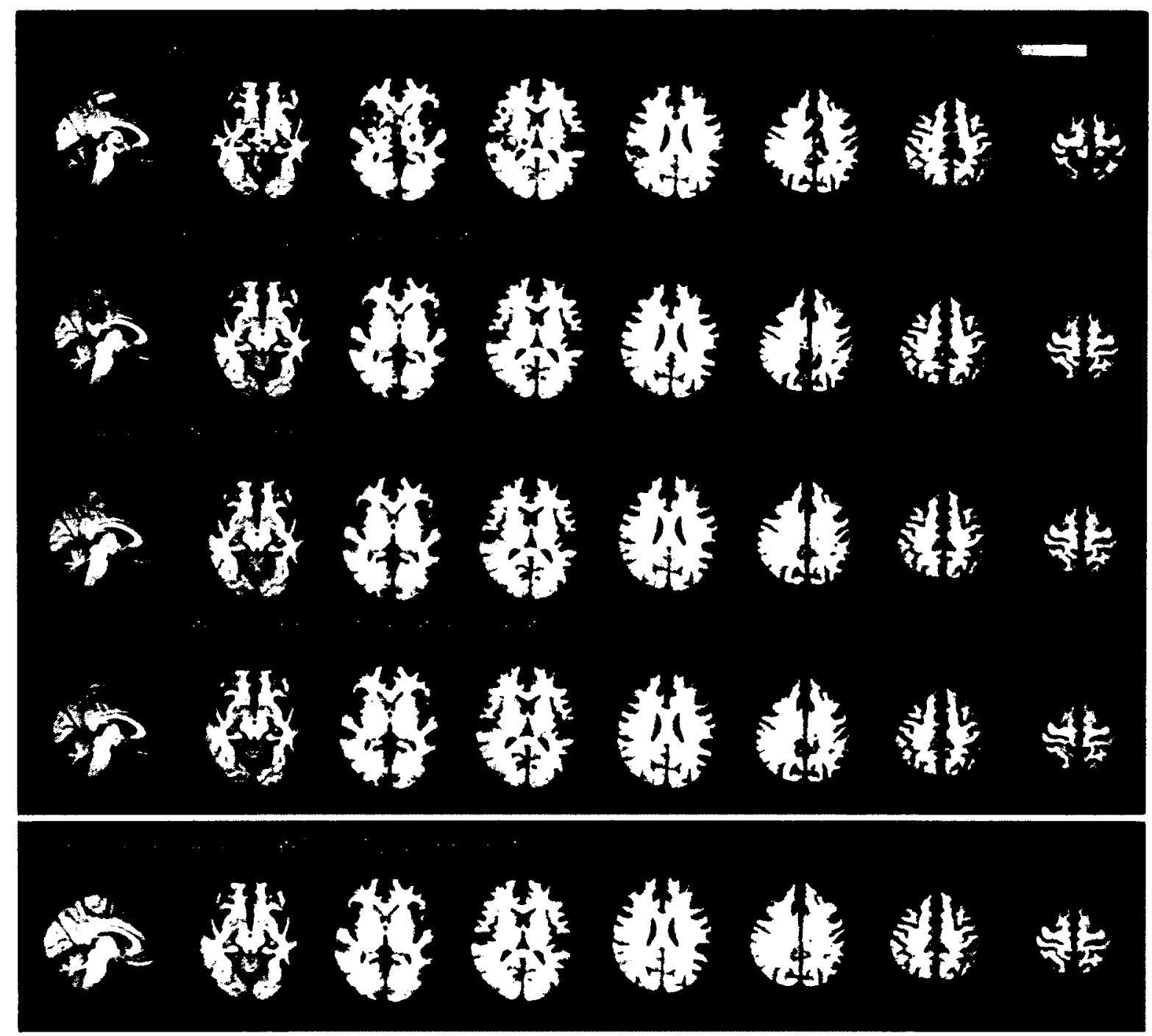

Figure E.2: Brain maps showing (A) the basic contrast of the anticipation of uncertain aversion - safe [AUA Safe]: (B) the negative correlation between [ALA Safe] contrast values and CTQ scores: $(\mathrm{C})$ the positive correlation between $[\mathrm{ACA}$ Safe] contrast values and mPFC Glu/NAA: (D) the negative correlation between [AUA Safe] contrast values and mPFC entropy: and (E) the overlap, in green, between each of these maps. Results are thresholded at $p \cdot 0.05$, FWE corrected $(\mathrm{n}=14)$, other than [ALA Safe] rs mPFC Glu which is thresholded at $p \quad 0.005$, uncorrected. Results are shown superimposed on the study mean anatomical image. Sagittal $x=6$. 


\begin{tabular}{|c|c|c|c|c|}
\hline Region & & $x, y, z$ & voxels & Z-max \\
\hline \multirow[t]{4}{*}{$\mathrm{SMC}$} & $\mathrm{R}$ & $10-3466$ & 6317 & 4.9 \\
\hline & & $22-3874$ & & \\
\hline & $L$ & $-14-3264$ & & \\
\hline & & $-18-4466$ & & \\
\hline \multirow[t]{2}{*}{$\mathrm{MCC} / \mathrm{SMA}$} & $\mathrm{L} / \mathrm{R}$ & $4-1444$ & & \\
\hline & & $8-636$ & & \\
\hline $\mathrm{ACC}$ & $\mathrm{L} / \mathrm{R}$ & -22024 & & \\
\hline \multirow{5}{*}{$\begin{array}{l}\text { post-insula/HG } \\
\text { mid-temporal gyrus } \\
\text { ant-insula/operculum }\end{array}$} & $\mathrm{R}$ & $\begin{array}{llll}36 & -20 & 12\end{array}$ & 4830 & 4.92 \\
\hline & & $66-36-10$ & & \\
\hline & & $\begin{array}{llll}48 & 20 & -4\end{array}$ & & \\
\hline & & 38280 & & \\
\hline & & 60204 & & \\
\hline \multirow[t]{2}{*}{ thalamus } & $\mathrm{R}$ & $10-12-2$ & & \\
\hline & $\mathrm{L}$ & $-18-24-4$ & & \\
\hline \multirow[t]{2}{*}{ mid-insula } & $\mathrm{R}$ & 3840 & & \\
\hline & & $40 \quad 0-12$ & & \\
\hline \multirow[t]{2}{*}{ mid-brain } & $L / R$ & $-6-22-10$ & & \\
\hline & & $8-28-10$ & & \\
\hline \multirow[t]{2}{*}{ VS } & $\mathrm{R}$ & $2814-2$ & & \\
\hline & & 1022 & & \\
\hline \multirow[t]{6}{*}{ mid-insula } & $\mathrm{L}$ & -3840 & 1938 & 4.15 \\
\hline & & $\begin{array}{lll}-34 & -24 & 14\end{array}$ & & \\
\hline & & $\begin{array}{lll}-52 & -32 & 20\end{array}$ & & \\
\hline & & -44164 & & \\
\hline & & $-364-18$ & & \\
\hline & & -6080 & & \\
\hline post-SMG & & $\begin{array}{lll}-50 & -48 & 32\end{array}$ & & \\
\hline VS & & -1666 & & \\
\hline \multirow[t]{3}{*}{ Cerebellum } & $\mathrm{L} / \mathrm{R}$ & $0-52-8$ & 1033 & 4.26 \\
\hline & & $-8-62-42$ & & \\
\hline & & $10-66-22$ & & \\
\hline Lat-OC & $\mathrm{R}$ & $48-6046$ & 722 & 3.76 \\
\hline SMG & & $60-4626$ & & \\
\hline
\end{tabular}

Table E.1: Results for contrast [AUA > Safe]. Coordinates are in MNI space. Significance threshold is $p<0.05$, FWE corrected $(\mathrm{Z}>2.3)$. SMC = sensory-motor cortex; $\mathrm{SMA}=$ supplementary motor area; $\mathrm{HG}=$ Herschl's gyrus; $1^{\circ} \mathrm{MC}=$ primary motor cortex; $2^{\circ}$ visual $=$ secondary visual cotex; $\mathrm{ACC}=$ anterior cingulate cortex POC $=$ parietal operculum cortex; VS = ventral striatum; $\mathrm{MCC}=$ midcingulate cortex; $\mathrm{ACC}=$ anterior cingulate cortex; $\mathrm{PCC}=$ posterior cingulate cortex; lat-OC = lateral occipital cortex; post-SMG = posterior supramarginal gyrus 


\begin{tabular}{|c|c|c|c|c|}
\hline Region & & $x, y, z$ & voxels & Z-max \\
\hline $\mathrm{PCC} /$ precuneus & $\mathrm{L} / \mathrm{R}$ & $-10-4440$ & 2222 & 3.35 \\
\hline
\end{tabular}

Table E.2: Cluster locations for [AUA vs Safe] vs CTQ regression. Coordinates are in MNI space. Threshold is $p<0.05$, FWE corrected $(\mathrm{Z}>2.3)$. $\mathrm{PCC}=$ posterior cingulate cortex.

\begin{tabular}{ccccc} 
Region & & $\mathrm{x}, \mathrm{y}, \mathrm{z}$ & voxels & $\mathrm{Z}-\max$ \\
\hline SMC/precuneus/PCC & $\mathrm{L} / \mathrm{R}$ & $2-1650$ & 1465 & 3.19
\end{tabular}

Table E.3: Cluster locations for [AUA vs Safe] vs mPFC Ent regression. Coordinates are in MNI space. Threshold is $p<0.05$, FWE corrected $(Z>2.3)$. SMC $=$ sensory-motor cortex; $\mathrm{PCC}=$ posterior cingulate cortex. 